\title{
Test Scenarios for Mission Critical Push-To-Talk (MCPTT) Off-Network Mode Protocols Implementation
}

\author{
Priam Varin \\ Yishen Sun \\ Wesley Garey
}

This publication is available free of charge from: https://doi.org/10.6028/NIST.IR.8236 


\title{
Test Scenarios for Mission Critical Push-To-Talk (MCPTT) Off-Network Mode Protocols Implementation
}

\author{
Priam Varin \\ Yishen Sun \\ Wesley Garey \\ Wireless Networks Division \\ Communications Technology Laboratory
}

This publication is available free of charge from:

https://doi.org/10.6028/NIST.IR.8236

October 2018

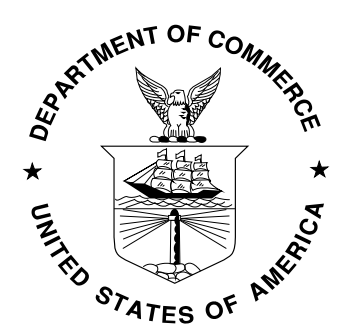

U.S. Department of Commerce Wilbur L. Ross, Jr., Secretary

National Institute of Standards and Technology Walter Copan, NIST Director and Undersecretary of Commerce for Standards and Technology 


\begin{abstract}
The document provides both an overview and technical details of test scenarios that are designed by our group. These test scenarios can be used to check the protocol implementation of the 3rd Generation Partnership Project (3GPP) defined off-network mode operations of mission critical push-to-talk (MCPTT), covering floor control, call control, and call type control. This report includes test scenarios for basic group calls, broadcast group calls, and private calls. A test scenario is presented in a table format, and sometimes is accompanied by a figure that shows the protocol messages exchanged over the air as well.
\end{abstract}

\title{
Keywords
}

MCPTT; off-network; basic group call; broadcast group call; private call; call control; floor control; test scenarios 


\section{Table of Contents}

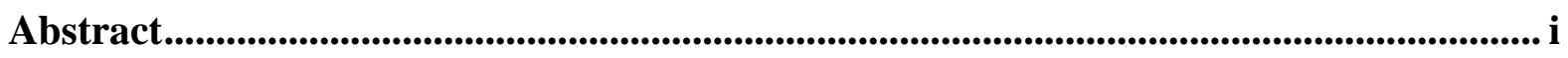

Keywords .................................................................................................................................. $\mathrm{i}$

1. Introduction ............................................................................................................................. 1

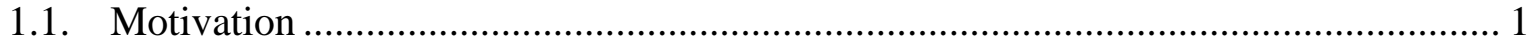

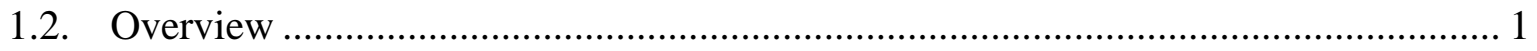

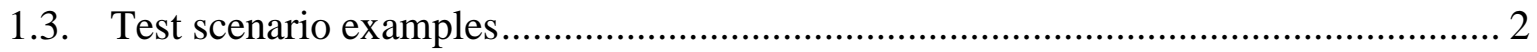

2. Test scenarios details - basic group call .................................................................................. 6

2.1. Basic group call - floor control ......................................................................... 6

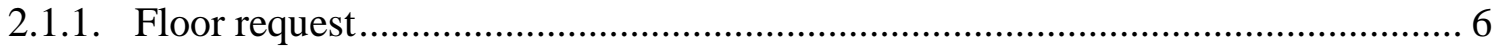

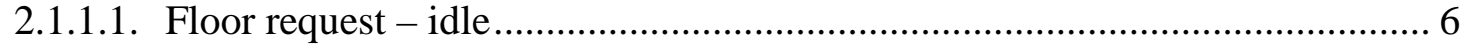

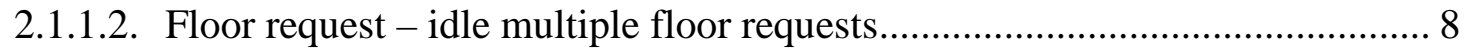

2.1.1.3. Floor request - queued ............................................................................... 14

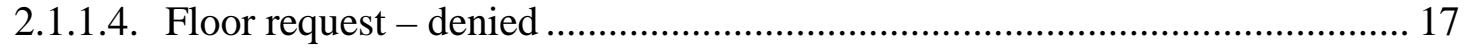

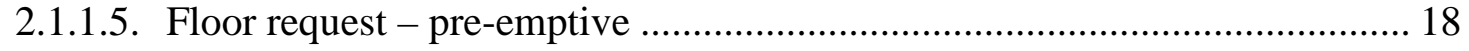

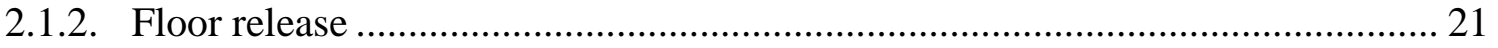

2.1.2.1. Floor release by the floor arbitrator............................................................. 21

2.1.2.2. Floor release by a queued floor participant .................................................... 22

2.1.2.3. Floor release by the floor arbitrator with queued participants ....................... 23

2.1.2.4. Floor release by pre-empted floor arbitrator ................................................. 25

2.1.3. Session initialization.................................................................................. 28

2.1.3.1. Session initialization - normal ................................................................. 28

2.1.3.2. Session initialization - message lost .......................................................... 30

2.1.3.3. Session initialization - private call.............................................................. 32

2.1.3.4. Session initialization - broadcast call ........................................................... 34

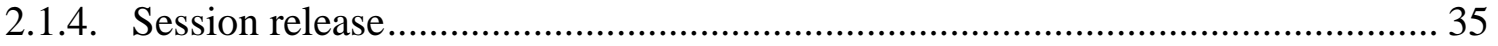

2.2. Basic group call - call control ....................................................................... 36

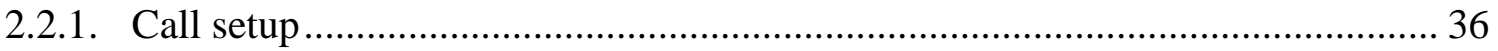

2.2.1.1. Call setup - join a call ............................................................................... 36

2.2.1.2. Call setup - establish a new call with confirm mode indication IE in the Call Announcement message............................................................................................. 39

2.2.1.3. Call setup - establish a new call without confirm mode indication IE in the Call Announcement message .................................................................................... 44

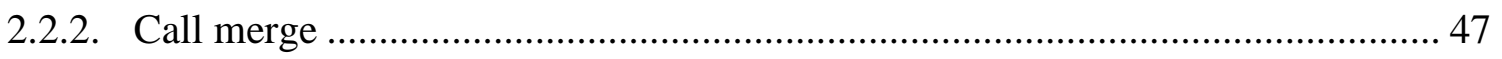

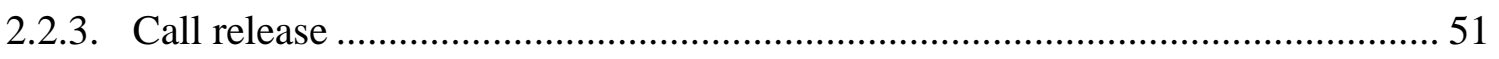




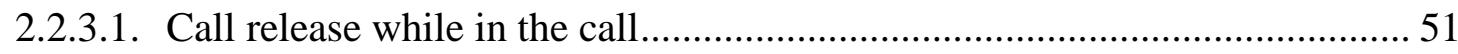

2.2.3.2. Call release after call probe and return to "S1: start-stop" ............................. 53

2.2.3.3. Call release after call probe and enter "S6: ignoring incoming call

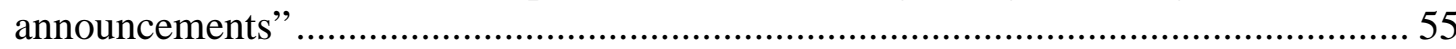

2.2.3.4. Call release while pending user action - without confirm indication ............ 56

2.2.3.5. Call release while pending user action - with confirm indication ................ 57

2.2.3.6. Call release when maximum duration of the call is reached .......................... 59

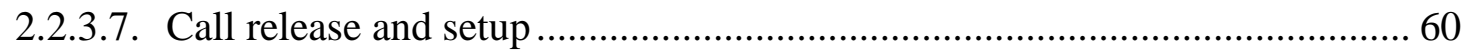

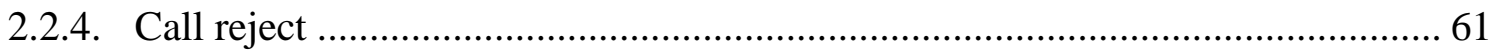

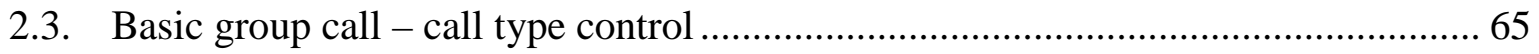

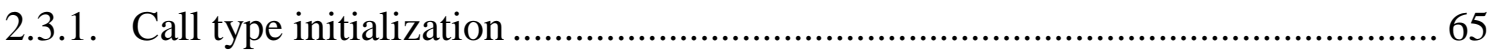

2.3.1.1. Call type initialization - establish a new basic call ........................................ 65

2.3.1.2. Call type initialization - establish a new emergency call .............................. 66

2.3.1.3. Call type initialization - establish a new imminent peril call ......................... 67

2.3.1.4. Call type initialization - join an emergency call after call probe................... 68

2.3.1.5. Call type initialization - join an imminent peril call after call probe............. 69

2.3.1.6. Call type initialization - join a basic group call after group call probe ........ 70

2.3.1.7. Call type initialization - join a basic group call with user acknowledgement

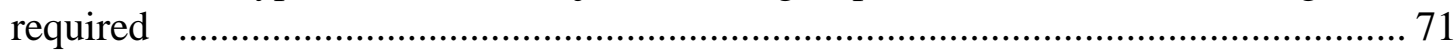

2.3.1.8. Call type initialization - join an imminent peril group call with user acknowledgement required ...................................................................................... 72

2.3.1.9. Call type initialization - join an emergency group call with user acknowledgement required ......................................................................................... 73

2.3.1.10. Call type initialization - join an emergency group call without user acknowledgement required ........................................................................................... 74

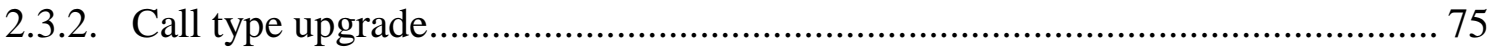

2.3.2.1. Call upgrade from basic to imminent peril group call................................... 75

2.3.2.2. Call upgrade from basic to emergency group call......................................... 76

2.3.2.3. Call upgrade from imminent peril group call................................................ 77

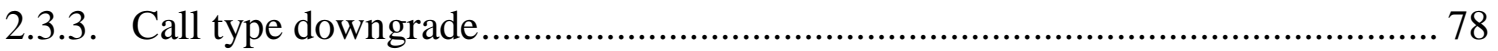

2.3.3.1. Explicit downgrade from emergency call ................................................... 78

2.3.3.2. Explicit downgrade from imminent peril call ................................................. 80

2.3.3.3. Implicit downgrade from emergency call ..................................................... 82

2.3.3.4. Implicit downgrade from imminent peril call ................................................ 83

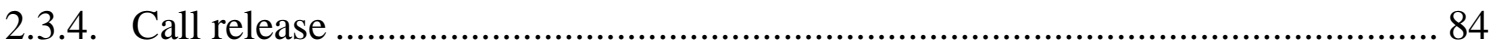

2.3.4.1. Call release after call establishment .............................................................. 84 
2.3.4.2. Call release before call establishment ........................................................... 85

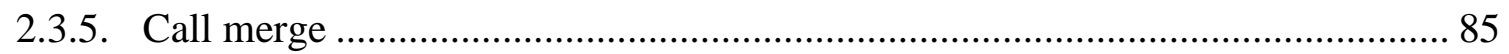

2.3.5.1. Call merge from different call type states ..................................................... 85

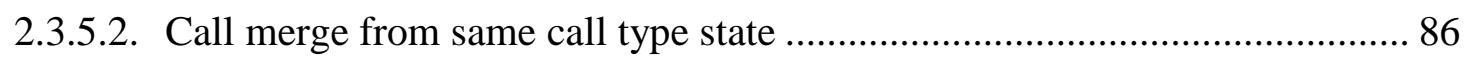

3. Test scenarios details - broadcast group call.................................................................... 88

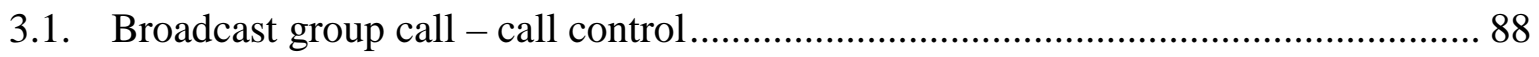

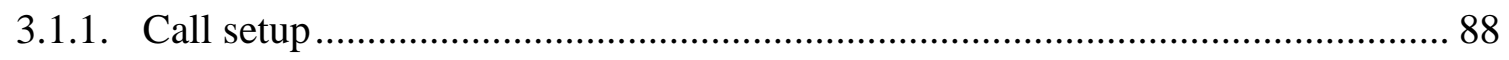

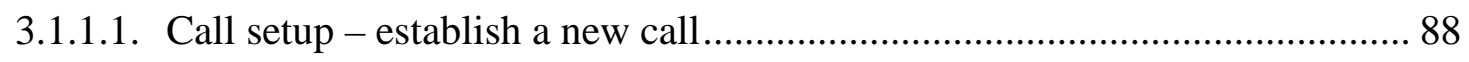

3.1.1.2. Call setup - establish a new call with refusal to join .................................... 89

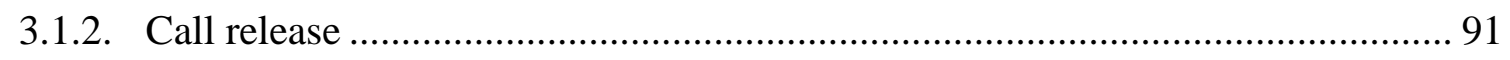

3.1.2.1. Call release by the originating user ............................................................... 91

3.1.2.2. Call release by a participant ........................................................................ 92

4. Test scenarios details - private call.................................................................................... 95

4.1. Private call - call control ................................................................................... 95

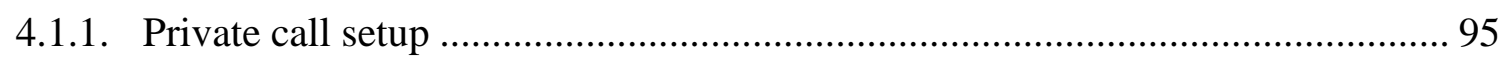

4.1.1.1. Private call setup - establish new call in automatic mode ............................ 95

4.1.1.2. Private call setup - establish and cancel new call in automatic mode .......... 97

4.1.1.3. Private call setup - establish new call in manual mode ............................... 99

4.1.1.4. Private call setup - establish and cancel new call in manual mode ............. 101

4.1.1.5. Private call setup - failure to establish new call ........................................ 103

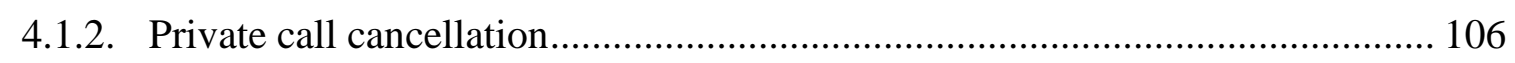

4.1.2.1. Private call cancellation - cancel an ongoing call (normal)........................ 106

4.1.2.2. Private call cancellation - cancel an ongoing call (timer expiry) ............... 106

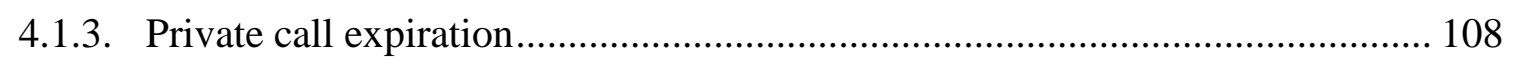

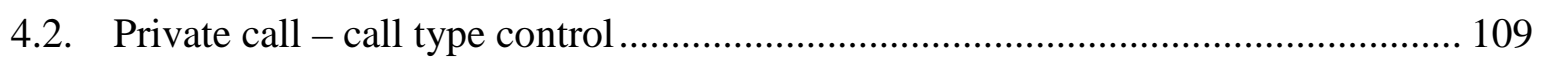

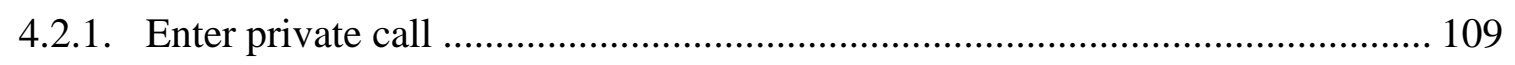

4.2.2. Enter private emergency call ...................................................................... 111

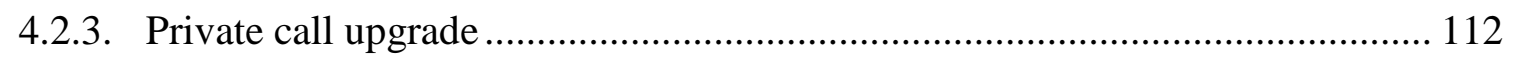

4.2.3.1. Private call upgrade - upgrade call ........................................................... 112

4.2.3.2. Private call upgrade - rejected upgrade call................................................ 113

4.2.3.3. Private call upgrade - failed upgrade call .................................................... 115

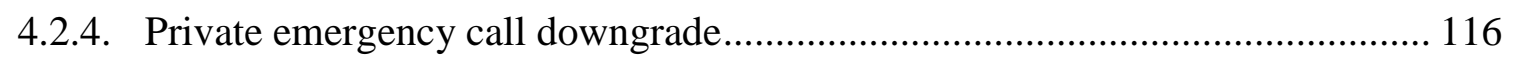

4.2.4.1. Private emergency call downgrade - downgrade call TFP6 expiry ............. 116

4.2.4.2. Private emergency call downgrade - downgrade call out of range.............. 118 
4.2.4.3. Private emergency call downgrade - downgrade call TFP8 expiry 120

5. Summary and future work ............................................................................ 122

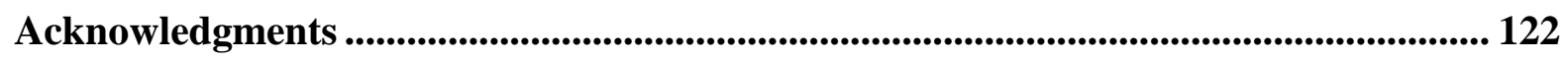

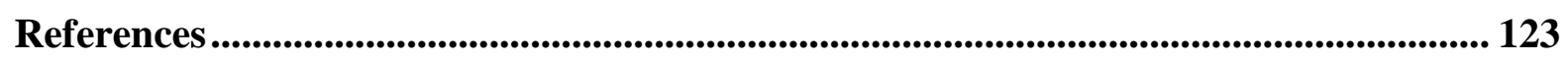

Appendix: Scenario usages............................................................................... 125 


\section{List of Tables}

Table 1: Floor release, 2 UEs (example)........................................................................ 2

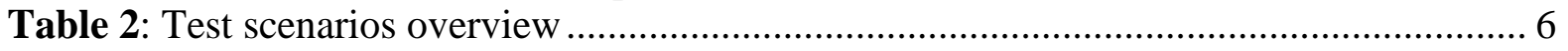

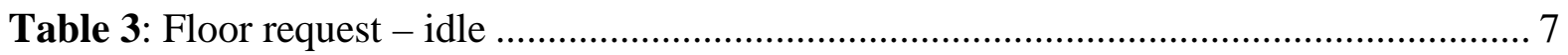

Table 4: Floor request - idle multiple floor requests ............................................................... 9

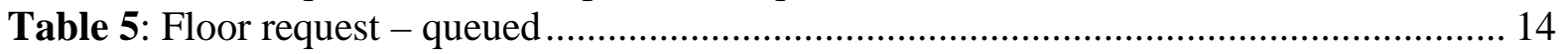

Table 6: Floor request - denied.................................................................................. 18

Table 7: Floor request - pre-emptive ................................................................................. 19

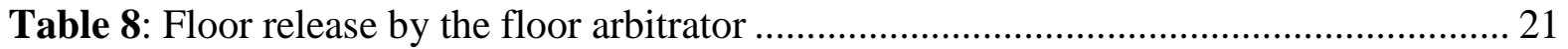

Table 9: Floor release by a queued floor participant.............................................................. 22

Table 10: Floor release by the floor participant with queued participants............................... 23

Table 11: Floor release by pre-empted floor arbitrator …………………………….............. 25

Table 12: Session initialization - normal....................................................................... 29

Table 13: Session initialization - message lost ................................................................. 30

Table 14: Session initialization - private call ………….................................................. 32

Table 15: Session initialization - broadcast call ................................................................... 35

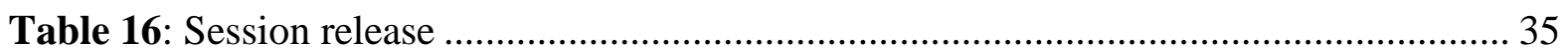

Table 17: Call setup - join a call........................................................................................ 36

Table 18: Call setup - establish a new call with confirm mode indication IE in the Call

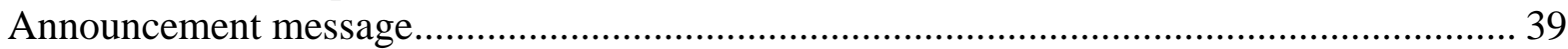

Table 19: Call setup - establish a new call without confirm mode indication IE in the Call

Announcement message................................................................................................. 45

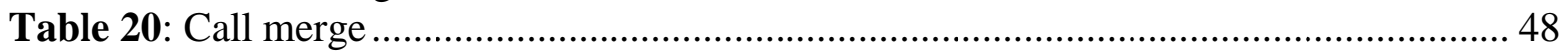

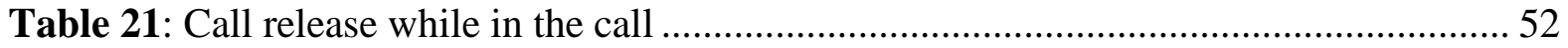

Table 22: Call release after call probe and return to "S1: start-stop" ................................... 54

Table 23: Call release after call probe and enter "S6: ignoring incoming call announcements"

Table 24. Call release while pending user action - without confirm indication..............55

Table 25: Call release while pending user action - with confirm indication ............................5 58

Table 26: Call release when maximum duration of the call is reached.................................. 60

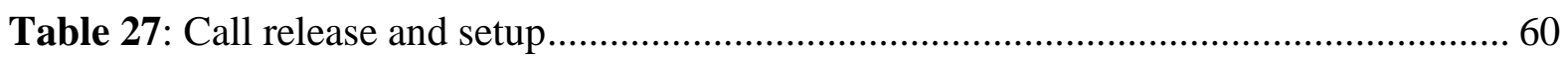

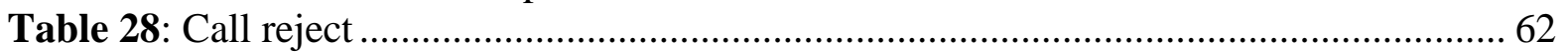

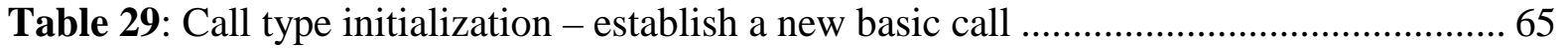

Table 30: Call type initialization - establish a new emergency call ....................................... 66

Table 31: Call type initialization - establish a new imminent peril call ................................... 67

Table 32: Call type initialization - join an emergency call after call probe ............................ 68

Table 33: Call type initialization - join an imminent peril call after GROUP CALL PROBE

Table 34: Call type initialization - join a basic group call after group call probe .................. 70

Table 35: Call type initialization - join a basic group call with user acknowledgement

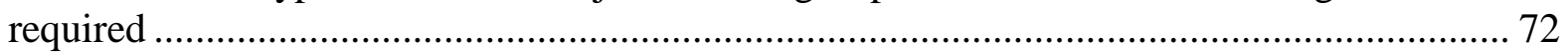

Table 36: Call type initialization - join an imminent peril group call with user

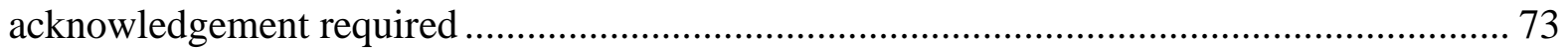
Table 37: Call type initialization - join an emergency group call with user acknowledgement required 
Table 38: Call type initialization - join an emergency group call without user

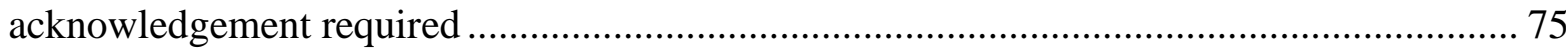

Table 39: Call upgrade from basic to imminent peril group call ........................................ 75

Table 40: Call upgrade from basic to emergency group call ............................................ 76

Table 41: Call upgrade from imminent peril group call................................................. 77

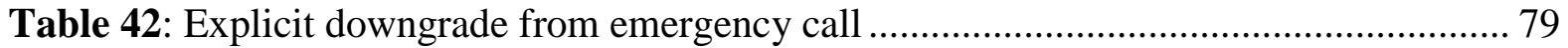

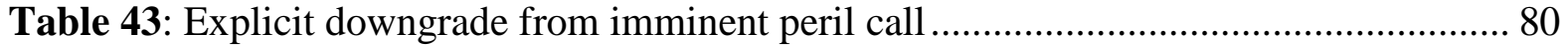

Table 44: Implicit downgrade from emergency call ................................................... 83

Table 45: Implicit downgrade from imminent peril call ............................................. 83

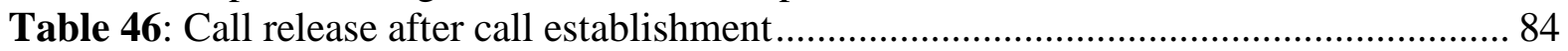

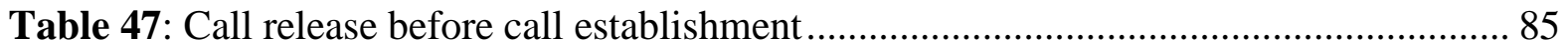

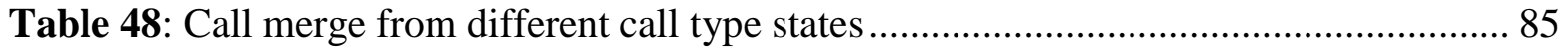

Table 49: Call merge from same call type state ............................................................ 86

Table 50: Call setup - establish a new call ............................................................... 88

Table 51: Call setup - establish a new call with refusal to join ........................................ 89

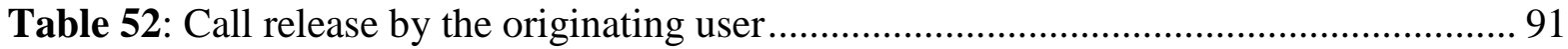

Table 53: Call release by a participant ................................................................... 92

Table 54: Private call setup - establish new call in automatic mode................................. 95

Table 55: Private call setup - establish and cancel new call in automatic mode .................. 97

Table 56: Private call setup - establish new call in manual mode .................................... 100

Table 57: Private call setup - establish and cancel new call in manual mode.................... 102

Table 58: Private call setup - failure to establish a new call ........................................... 103

Table 59: Private call cancellation - cancel an ongoing call (normal) ............................. 106

Table 60: Private call cancellation - cancel an ongoing call (timer expiry) ...................... 107

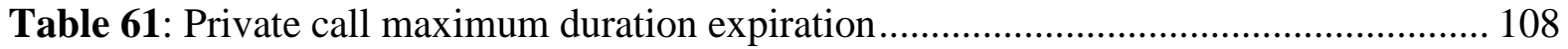

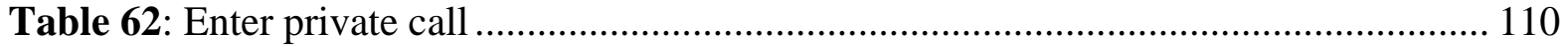

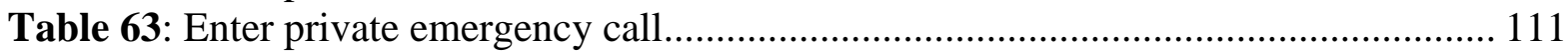

Table 64: Private call upgrade - upgrade call ............................................................. 112

Table 65: Private call upgrade - rejected upgrade call ................................................. 114

Table 66: Private call upgrade - failed upgrade call ...................................................... 115

Table 67: Private emergency call downgrade - downgrade call TFP6 expiry................... 117

Table 68: Private emergency call downgrade - downgrade call out of range ..................... 119

Table 69: Private emergency call downgrade - downgrade call TFP8 expiry.................... 120 


\section{List of Figures}

Figure 1: Floor request - deny, 2 UEs (example) ……………………............................. 5

Figure 2: Floor request - idle......................................................................................... 8

Figure 3: Floor request - idle multiple floor requests............................................................ 13

Figure 4: Floor request - queued ............................................................................... 17

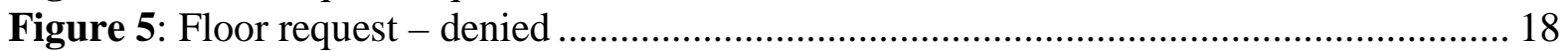

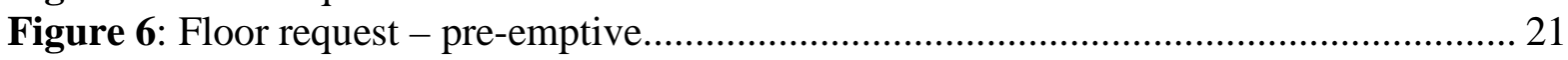

Figure 7: Floor release by pre-empted floor arbitrator ........................................................ 28

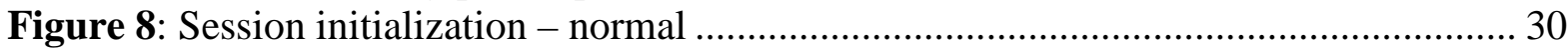

Figure 9: Session initialization - message lost .................................................................. 31

Figure 10: Session initialization - private call..................................................................... 34

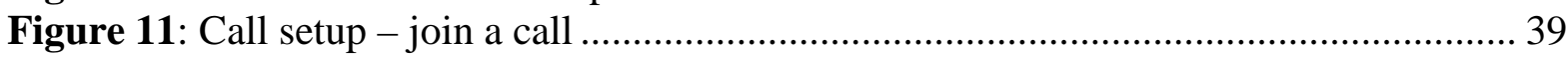

Figure 12: Call setup - establish a new call with confirm mode .......................................... 44

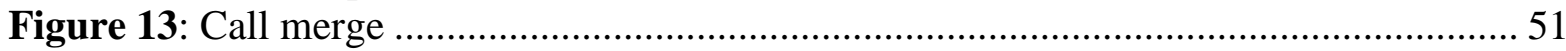

Figure 14: Broadcast call setup - establish a new call with refusal to join ............................ 91

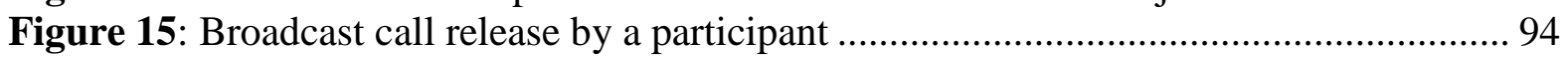

Figure 16: Private call setup - establish new call in automatic mode ................................... 96

Figure 17: Private call setup - establish and cancel new call in automatic mode ................. 99

Figure 18: Private call setup - establish new call in manual mode ..................................... 101

Figure 19: Private call setup - establish and cancel new call in manual mode .................... 103

Figure 20: Private call setup - failure to establish a new call.............................................. 105

Figure 21: Private call cancellation - cancel an ongoing call (timer expiry) ....................... 108

Figure 22: Private call upgrade - rejected upgrade call........................................................ 115

Figure 23: Private emergency call downgrade - downgrade call TFP6 expiry ………........ 118

Figure 24: Private emergency call downgrade - downgrade call out of range ..................... 120 


\section{Introduction}

\subsection{Motivation}

Public safety agencies require real-time applications. Mission critical voice (MCV) real-time application remains the most critical means of communications for first responders in emergency situations and cannot be compromised. The major MCV communication device is using the mission critical push-to-talk (MCPTT) technology, which achieves public safety grade PTT voice quality. A few general public safety requirements/expectations of push-totalk communications over Long Term Evolution (LTE) may be found in [1]. The 3rd Generation Partnership Project (3GPP) working groups have defined a set of specifications [27] for MCPTT operations over LTE in its recent releases: Release-13, Release-14, and Release 15, for both on-network mode and off-network mode.

When mission critical voice communication is desired while first responders are out of LTE network coverage or when the network coverage is very weak, it is a very attractive or the only feasible option for public safety users to operate in MCPTT off-network mode. However, due to the lack of time or effort spent on off-network scenarios by the 3GPP working groups, the completeness and correctness of MCPTT off-network mode are yet to be fully verified. In fact, although the relevant 3GPP Release-13 specifications were officially completed in March 2016, quite a number of change requests (CRs) were submitted to LTE work groups to correct errors. New features and enhancements of existing functionalities were discussed for Release14 and Release 15, as well. Therefore, evaluating the performance and limitations of MCPTT off-network operations is of primary interest to the public safety community. For this reason, it is one of the main research focuses of our division. Unless noted otherwise, the MCPTT protocols, research findings, or both that will be discussed in the remainder of the document all refer to MCPTT off-network mode.

Since the spring of 2016, our group has been developing an MCPTT off-network mode model in ns-3. Major features of this model include basic group call capability, broadcast group call capability, private call capability, floor control, call control, call type control, and emergency alert. In order to check the correctness of protocol implementation defined in that ns-3 model, our group has designed 61 test scenarios for MCPTT off-network mode operations. Future revisions of this document may include additional scenarios. These test scenarios were used to check our implementation of the MCPTT off-network mode protocols using ns-3 simulation tool. As can be seen from the example shown in Section 1.3, as well as throughout the remaining document, these test scenarios are based on the 3GPP MCPTT off-network mode protocols and are not ns-3 specific. Therefore, there are potential benefits of utilizing these test scenarios to verify the MCPTT off-network mode protocol implementations in other embodiments, e.g., prototype or commercial devices. Utilizing the information provided in these scenarios, one could create conformance tests or interoperability tests, as shown through examples in the Appendix.

\subsection{Overview}

The remainder of the document is organized as follows. Example test scenarios are given in Section 1.3, so that the interpretation and utilization of a test scenario may be illustrated in 
more details. Section 2 is the collection of all test scenarios that our group has designed for MCPTT off-network mode basic group call, including 14 test scenarios on floor control (Section 2.1), 12 test scenarios on call control (Section 2.2), and 21 test scenarios on call type control (Section 2.3). Section 3 summarizes all test scenarios we designed for MCPTT offnetwork broadcast group call, including 4 scenarios on call control (Section 3.1). Section 4 presents test scenarios developed for MCPTT off-network private call, including 8 scenarios on call control (Section 4.1) and 8 scenarios on call type control (Section 4.2). Summary and future work is discussed in Section 5.

Test scenarios are designed to cover possible state transitions, as well as the main procedures of MCPTT off-network mode operations.

\subsection{Test scenario examples}

In this section, a test scenario example will be explained in detail, in order to help the interpretation and utilization of other test scenarios listed in the remainder of the document.

Each test scenario is presented in a table format. The table captures important information that needs to be checked for each test scenario, such as event triggers, sequences, message exchanges, timers, counters, and states. In addition to the table, a corresponding figure is included in some of the test scenarios. The figure is provided to highlight the sequence of MCPTT messages exchanged over the air, and thus can be used as a reference for the design of 3GPP working group, RAN5, test scenarios. Note that the information contained in a figure is a subset of information already included in the table of the same scenario. However, a figure provides a better visualization of the interaction between users and the timeline of events. For those test scenarios where there are none or very few over-the-air messages involved, a figure is not shown.

As an example, Table 1 is the corresponding table of the "Floor Release" test scenario with 2 User Equipments (UEs) in the MCPTT group call. The $1^{\text {st }}$ row names the columns, which will be explained in more details later in this section. The $2^{\text {nd }}$ row specifies the initial states of each UE when the test scenario starts. Starting from the $3^{\text {rd }}$ row, the table lists a sequence of events organized chronologically, from the earliest (at the top of the table) to the latest (at the bottom of the table). Each row corresponds to an event/step of the test scenario, and there are 2 steps in this example, shown as $3^{\text {rd }}$ and $4^{\text {th }}$ row in Table 1.

Table 1: Floor release, 2 UEs (example)

\begin{tabular}{|c|c|c|c|c|c|c|}
\hline & Timeline & Triggering event & $\begin{array}{c}\text { UE A state } \\
\text { transition }\end{array}$ & $\begin{array}{c}\text { UE B state } \\
\text { transition }\end{array}$ & $\begin{array}{c}\text { Consequent } \\
\text { behavior }\end{array}$ & $\begin{array}{c}\text { Reference } \\
\text { for details }\end{array}$ \\
\hline 0 & - & - & $\begin{array}{c}\text { O: has } \\
\text { permission }\end{array}$ & $\begin{array}{c}\text { O: has no } \\
\text { permission }\end{array}$ & - & - \\
\hline
\end{tabular}




\begin{tabular}{|c|c|c|c|c|c|c|}
\hline & Timeline & Triggering event & $\begin{array}{l}\text { UE A state } \\
\text { transition }\end{array}$ & $\begin{array}{l}\text { UE B state } \\
\text { transition }\end{array}$ & $\begin{array}{c}\text { Consequent } \\
\text { behavior }\end{array}$ & $\begin{array}{l}\text { Reference } \\
\text { for details }\end{array}$ \\
\hline 1 & T0 & $\begin{array}{c}\text { UE A receives } \\
\text { MCPTT } \\
\text { indication to stop } \\
\text { sending the Real- } \\
\text { time Transport } \\
\text { Protocol (RTP) } \\
\text { packets } \\
\text { (No Queued Floor } \\
\text { Requests at UE A) }\end{array}$ & O: silence & - & $\begin{array}{c}\text { UE A sends Floor } \\
\text { Release, starts T230, } \\
\text { stops T206 and T207 } \\
\text { (if running), and } \\
\text { clears } \\
\text { Synchronization } \\
\text { SouRCe (SSRC) of } \\
\text { current arbitrator }\end{array}$ & 7.2.3.5.5 \\
\hline 2 & $\begin{array}{l}\mathrm{T} 0+ \\
\mathrm{t} \_\mathrm{AB} 1\end{array}$ & $\begin{array}{l}\text { UE B receives } \\
\text { Floor Release }\end{array}$ & - & O: silence & $\begin{array}{c}\text { UE B stops } \\
\text { rendering RTP } \\
\text { packets, stops T203 } \\
\text { (if running), starts } \\
\text { T230, may send } \\
\text { Floor Idle } \\
\text { notification to } \\
\text { MCPTT user, and } \\
\text { clears SSRC of } \\
\text { current arbitrator }\end{array}$ & 7.2.3.4.3 \\
\hline
\end{tabular}

Each column of the table focuses on a specific aspect of the test scenario:

- The first unlabeled column numbers the rows in the table for easy referencing. The row labeled zero provides the initial starting states for each scenario. The row numbers provide the ordering of the events/steps in the scenario.

- "Timeline" is the timing of the event described in the row. T0 denotes the start time of every scenario, and the timing of each event is indicated in the form of a time increment in addition to the timing of the previous event wherever possible, e.g., T0 + $\mathrm{t}$-AB1. The letters $\mathrm{A}, \mathrm{B}$ or $\mathrm{C}$ in the time increment symbol indicate the main contributor of the time increment. t_ABx represents the transmission delay from UE A to UE B. t_BAx represents the transmission delay from UE B to UE A. t_Ax represents the time between T0 and the moment the stated action is performed at UE A. The " $x$ " represents the ordered instance, e.g., $t$ _AB 1 is the first instance of the transmission delay between UE A and UE B and t_AB2 is the second instance of the transmission delay between UE A and UE B.

- "Triggering event" indicates the event that occurs at the time specified by "Timeline" of the same row. The triggering of such an event may result in a UE state transition, as well as consequent behavior, which are specified in other columns. A triggering event can be a timer expiration, a counter reaching its upper bound, the reception of a specific message (e.g., "UE B receives Floor Release”), the sending of RTP packets, etc. A triggering event may also include certain conditions that need to be met, e.g., "No Queued Floor Request at UE A" in row 3; such conditions shall be displayed between parentheses in an italic font.

- “UE A state transition” and "UE B state transition” indicate whether there are any changes in the UEs' states during this event, and if yes, to which state. In Table 1, UE A starts in the ' $O$ : has permission' state, and then transitions to the ' $O$ : silence' state after the first event. UE B starts in the 'O: has no permission' state, and then transitions to the 'O: silence' state after two events.

- "Consequent behavior" lists the actions that shall be taken immediately as the consequence of the "Triggering event". These actions focus on timer start/stop, counter maintenance, 
the type of control messages to be generated, the indication to MCPTT user, the handling of RTP packets in general, and the update of certain key parameters. Further details of how to set control message fields, update other parameters or both can be found by referring to the corresponding clause(s) provided in column "Reference for details". Note that if more than one action is present per UE, they are separated by commas. If there are more than one UE, a semicolon is used to separate the list of actions for UE from the list of actions for another UE. If several UEs share the same set of action(s) (e.g., share the same "Reference for details" clause), they may be grouped together.

- "Reference for details" provides references to the corresponding clause(s) in 3GPP specifications which are used as the basis when designing the test scenario and which are associated with that particular event. For floor control, test scenarios are designed based on 3GPP TS 24.380 v14.3.0 [5]. For call control and call type control, test scenarios are designed based on 3GPP TS 24.379 v14.2.0 [4]. These references are listed in the same order as that of the UEs described in the "Consequent behavior" section.

Additionally, throughout the table, several other color and font schemes are used:

- A UE represented in a bold font (such as "UE A") indicates that this UE is the main protagonist at play in a given cell description: in a "Triggering event" column, that means the embolden UE is the one for which we expect an action to occur before the scenario continues to unfold; in a "Consequent behavior" column, that means the text description represents the behavior for that embolden UE.

- A blue-font text indicates an MCPTT message (such as "Floor Request”)

- A green-font text indicates an MCPTT timer or counter (such as "T205” or "C205", respectively)

- Supplemental conditions and/or explanations can be encountered in various columns (such as already mentioned in the "Triggering event" column) by being displayed between parentheses using an italic font.

As another example, Fig. 1 is the figure corresponding to test scenario "Floor request - deny". The interpretation of a figure follows the following practice:

- State transitions of UEs, e.g., UE A and UE B in Fig. 1, are shown on the vertical axes.

- Events that occur during the tests scenario are organized temporally in a "top-to-bottom" fashion.

- Directional arrows between MCPTT clients represent messages exchanged between network entities, with the message name labeled. Arrows with solid lines indicate that messages are received successfully, whereas dotted lined arrows represent messages that cannot be received successfully by the MCPTT entity at the receiving UE, e.g., due to channel loss.

- Additional information may be included as well, such as timer start/stop, message preparation. Those action descriptions follow a color code: red for UE A, blue for UE B and orange for UE C. 


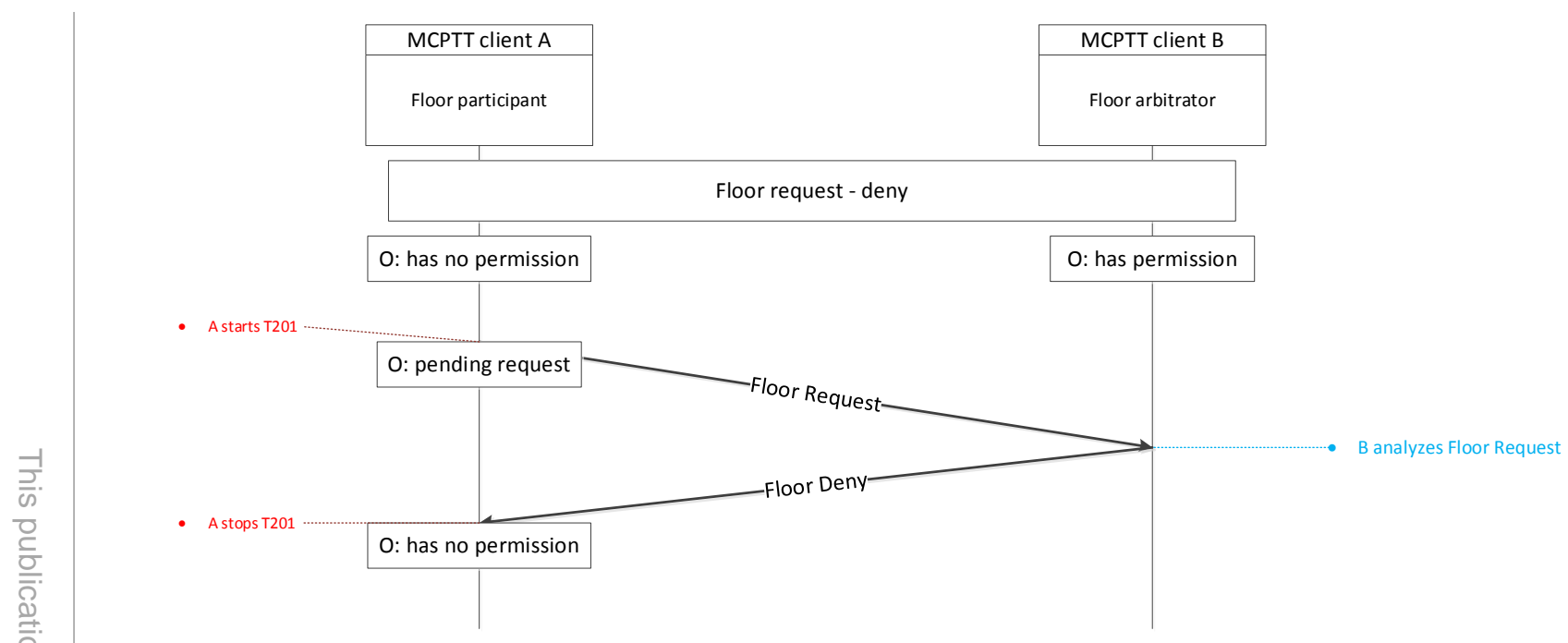

Figure 1: Floor request - deny, 2 UEs (example) 


\section{Test scenarios details - basic group call}

A brief overview of test scenarios to be discussed in this section is summarized by Table 2:

Table 2: Test scenarios overview

\begin{tabular}{|c|c|c|c|}
\hline Subject & Sub-area & $\begin{array}{c}\text { Number of } \\
\text { scenarios }\end{array}$ & $\begin{array}{l}\text { Reference 3GPP } \\
\text { specifications }\end{array}$ \\
\hline \multirow{4}{*}{$\begin{array}{l}\text { Basic Group Call } \\
\text { - Floor Control }\end{array}$} & Floor Request & 5 & \multirow[t]{4}{*}{ TS 24.380 v14.3.0 } \\
\hline & Floor Release & 4 & \\
\hline & Session Initialization & 4 & \\
\hline & Session Release & 1 & \\
\hline \multirow{4}{*}{$\begin{array}{l}\text { Basic Group Call } \\
\text { - Call Control }\end{array}$} & Call Setup & 3 & \multirow[t]{4}{*}{ TS 24.379 v14.2.0 } \\
\hline & Call Merge & 1 & \\
\hline & Call Release & 7 & \\
\hline & Call Reject & 1 & \\
\hline \multirow{5}{*}{$\begin{array}{l}\text { Basic Group Call } \\
\text { - Call Type } \\
\text { Control }\end{array}$} & Call Type Initialization & 10 & \multirow[t]{5}{*}{ TS 24.379 v14.2.0 } \\
\hline & Call Type Upgrade & 3 & \\
\hline & Call Type Downgrade & 4 & \\
\hline & Call Release & 2 & \\
\hline & Call Merge & 2 & \\
\hline \multirow{2}{*}{$\begin{array}{l}\text { Broadcast Group } \\
\text { Call - Call } \\
\text { Control }\end{array}$} & Call Setup & 2 & \multirow[t]{2}{*}{ TS 24.379 v14.2.0 } \\
\hline & Call Release & 2 & \\
\hline \multirow{3}{*}{$\begin{array}{l}\text { Private Call - } \\
\text { Call Control }\end{array}$} & Call Setup & 5 & \multirow[t]{3}{*}{ TS 24.379 v14.2.0 } \\
\hline & Call Cancellation & 2 & \\
\hline & Call Expiration & 1 & \\
\hline \multirow{4}{*}{$\begin{array}{l}\text { Private Call - } \\
\text { Call Type } \\
\text { Control }\end{array}$} & Enter Private Call & 1 & \multirow[t]{4}{*}{ TS 24.379 v14.2.0 } \\
\hline & Enter Private Emergency Call & 1 & \\
\hline & Private Call Upgrade & 3 & \\
\hline & Private Call Downgrade & 3 & \\
\hline
\end{tabular}

\subsection{Basic group call - floor control}

Floor control is the arbitration system in an MCPTT Service that determines who has the authority to transmit (talk) at a point in time during an MCPTT call [2].

The state transitions that are analyzed in each test scenario of Section 2.1 are based on Fig. 7.2.3.1-1 of [5]. Floor control test scenarios can be categorized into 4 groups: floor request, floor release, session initialization, and session release.

\subsubsection{Floor request}

\subsubsection{Floor request - idle}


This scenario describes the process of requesting the floor, with all (e.g., 3) participants idle, i.e., beginning in the ' $\mathrm{O}$ : silence' state. In this scenario, no other user intends to take the floor, except the one (UE A), which sends the floor request message. After multiple rounds of sending floor request messages, UE A assumes the floor. The other UEs' actions to the received floor control messages are shown.

Scenario Purpose: To observe that one and only one UE will become floor arbitrator and begin transmitting (talking) when there is currently no active floor arbitrator and the details of the protocol that allow this to happen.

Table 3: Floor request - idle

\begin{tabular}{|c|c|c|c|c|c|c|c|}
\hline & Timeline & $\begin{array}{c}\text { Triggering } \\
\text { event }\end{array}$ & $\begin{array}{l}\text { UE A state } \\
\text { transition }\end{array}$ & $\begin{array}{l}\text { UE B state } \\
\text { transition }\end{array}$ & $\begin{array}{l}\text { UE C state } \\
\text { transition }\end{array}$ & $\begin{array}{c}\text { Consequent } \\
\text { behavior }\end{array}$ & $\begin{array}{l}\text { Reference } \\
\text { for details }\end{array}$ \\
\hline 0 & - & - & O: silence & O: silence & O: silence & - & - \\
\hline 1 & T0 & $\begin{array}{c}\text { UE A } \\
\text { pushes PTT } \\
\text { button }\end{array}$ & $\begin{array}{c}\text { O: } \\
\text { pending } \\
\text { request }\end{array}$ & - & - & $\begin{array}{l}\text { UE A sends Floor } \\
\text { Request, stops } \\
\text { T230, starts T201 } \\
\text { and initializes } \\
\text { C201 }\end{array}$ & 7.2.3.3.2 \\
\hline 2 & $\begin{array}{l}\mathrm{T} 0+ \\
\mathrm{t} \_\mathrm{AB} 1\end{array}$ & $\begin{array}{l}\text { UE B and } \\
\text { UE C } \\
\text { receive } \\
\text { Floor } \\
\text { Request }\end{array}$ & - & - & - & $\begin{array}{l}\text { UE B and UE C } \\
\text { discard the floor } \\
\text { control message }\end{array}$ & $\begin{array}{r}\text { 7.2.3.1 } \\
\text { (7.2.3.3.5 } \\
\text { applicable } \\
\text { to private } \\
\text { call only) }\end{array}$ \\
\hline 3 & $\mathrm{~T} 0+\mathrm{T} 201$ & $\begin{array}{c}\text { UE A T201 } \\
\text { expires }\end{array}$ & - & - & - & $\begin{array}{c}\text { UE A resends } \\
\text { Floor Request, } \\
\text { starts T201 and } \\
\text { increments C201 }\end{array}$ & 7.2.3.6.9 \\
\hline 4 & $\begin{array}{c}\text { T0 + T201 } \\
+ \text { t_AB2 }\end{array}$ & $\begin{array}{l}\text { UE B and } \\
\text { UE C } \\
\text { receive } \\
\text { Floor } \\
\text { Request }\end{array}$ & - & - & - & $\begin{array}{l}\text { UE B and UE C } \\
\text { discard the floor } \\
\text { control message }\end{array}$ & $\begin{array}{r}\text { 7.2.3.1 } \\
\text { (7.2.3.3.5 } \\
\text { applicable } \\
\text { to private } \\
\text { call only) }\end{array}$ \\
\hline 5 & $\begin{array}{c}\mathrm{T} 0+ \\
2 * \mathrm{~T} 201\end{array}$ & $\begin{array}{c}\text { UE A T201 } \\
\text { expires }\end{array}$ & - & - & - & $\begin{array}{l}\text { UE A resends } \\
\text { Floor Request, } \\
\text { and starts T201 } \\
\text { and increments } \\
\quad \text { C201 }\end{array}$ & 7.2.3.6.9 \\
\hline
\end{tabular}




\begin{tabular}{|c|c|c|c|c|c|c|c|}
\hline & Timeline & $\begin{array}{c}\text { Triggering } \\
\text { event }\end{array}$ & $\begin{array}{l}\text { UE A state } \\
\text { transition }\end{array}$ & $\begin{array}{l}\text { UE B state } \\
\text { transition }\end{array}$ & $\begin{array}{l}\text { UE C state } \\
\text { transition }\end{array}$ & $\begin{array}{c}\text { Consequent } \\
\text { behavior }\end{array}$ & $\begin{array}{l}\text { Reference } \\
\text { for details }\end{array}$ \\
\hline 6 & $\begin{array}{c}\text { T0 + } \\
(\mathrm{n}-1) * \\
\text { T201 + } \\
\text { t_ABn }\end{array}$ & $\begin{array}{l}\text { UE B and } \\
\text { UE C } \\
\text { receive } \\
\text { Floor } \\
\text { Request } \\
\end{array}$ & - & - & - & $\begin{array}{l}\text { UE B and UE C } \\
\text { discard the floor } \\
\text { control message }\end{array}$ & $\begin{array}{r}\text { 7.2.3.1 } \\
\text { (7.2.3.3.5 } \\
\text { applicable } \\
\text { to private } \\
\text { call only) } \\
\end{array}$ \\
\hline 7 & $\begin{array}{c}\mathrm{T} 0+ \\
\mathrm{n} * \mathrm{~T} 201\end{array}$ & $\begin{array}{c}\text { UE A T201 } \\
\text { expires } \\
\text { (C201 at } \\
\text { upper limit) }\end{array}$ & $\begin{array}{c}\text { O: has } \\
\text { permission }\end{array}$ & - & - & $\begin{array}{c}\text { UE A sends Floor } \\
\text { Taken }\end{array}$ & 7.2.3.6.6 \\
\hline 8 & $\begin{array}{c}\mathrm{T} 0+ \\
\text { n*T201 + } \\
\text { t_AB } \\
(\mathrm{n}+1)\end{array}$ & $\begin{array}{l}\text { UE B and } \\
\text { UE C } \\
\text { receive } \\
\text { Floor Taken }\end{array}$ & - & $\begin{array}{l}\text { O: has no } \\
\text { permission }\end{array}$ & $\begin{array}{c}\text { O: has no } \\
\text { permission }\end{array}$ & $\begin{array}{c}\text { UE B and UE C } \\
\text { stop T230, start } \\
\text { T203 and set } \\
\text { SSRC of granted } \\
\text { floor arbitrator }\end{array}$ & 7.2.3.3.6 \\
\hline
\end{tabular}

And the corresponding figure is Fig. 2:

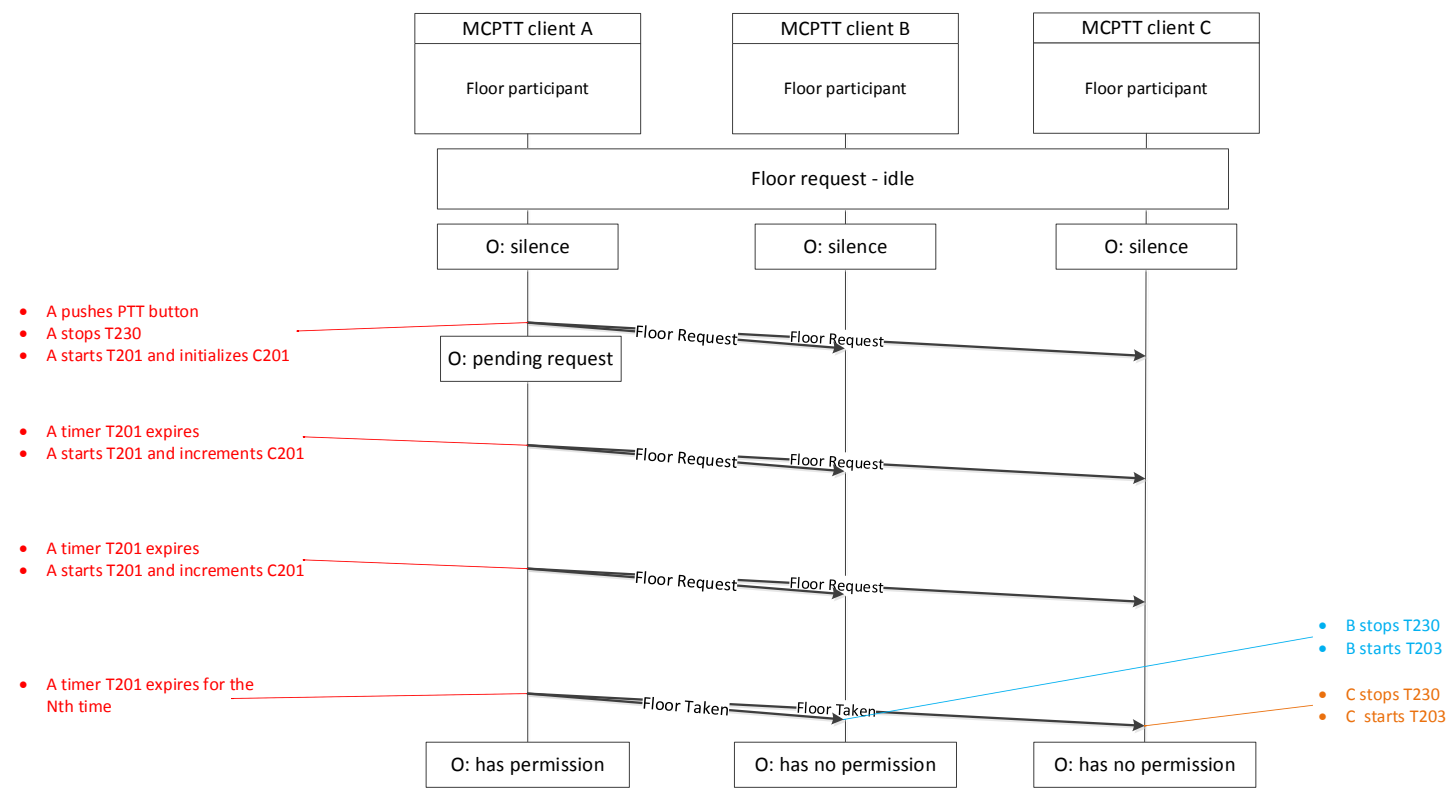

Figure 2: Floor request - idle

\subsubsection{Floor request - idle multiple floor requests}

This scenario describes the process of requesting the floor, in an alternative way compared to the previous scenario: all three participants begin in the ' $\mathrm{O}$ : silence' state and are at first not interested in taking the floor, hence transitioning to the 'O: start-stop' state. After a while, two users - UE A and UE C - declare their intention to take the floor. 
Scenario Purpose: To observe that a UE will become floor arbitrator when there is currently no active floor arbitrator and when two UEs requested the floor at a close time; to also observe that after being granted the floor and once done talking, the floor arbitrator tries to pass on the floor to the other queued user.

Table 4: Floor request - idle multiple floor requests

\begin{tabular}{|c|c|c|c|c|c|c|c|}
\hline & Timeline & $\begin{array}{c}\text { Triggering } \\
\text { event }\end{array}$ & $\begin{array}{l}\text { UE A state } \\
\text { transition }\end{array}$ & $\begin{array}{l}\text { UE B state } \\
\text { transition }\end{array}$ & $\begin{array}{l}\text { UE C state } \\
\text { transition }\end{array}$ & $\begin{array}{c}\text { Consequent } \\
\text { behavior }\end{array}$ & $\begin{array}{c}\text { Reference } \\
\text { for } \\
\text { details }\end{array}$ \\
\hline 0 & - & - & O: silence & O: silence & O: silence & - & - \\
\hline 1 & T0 & $\begin{array}{c}\text { UE A T230 } \\
\text { expires }\end{array}$ & $\begin{array}{l}\text { O: start- } \\
\text { stop }\end{array}$ & - & - & $\begin{array}{l}\text { UE A terminates } \\
\text { the floor } \\
\text { participant state } \\
\text { transition diagram }\end{array}$ & 7.2.3.3.7 \\
\hline 2 & $\begin{array}{l}\text { T0 + } \\
\text { t_B1 }\end{array}$ & $\begin{array}{c}\text { UE B T230 } \\
\text { expires }\end{array}$ & - & $\begin{array}{l}\text { O: start- } \\
\text { stop }\end{array}$ & - & $\begin{array}{l}\text { UE B terminates } \\
\text { the floor } \\
\text { participant state } \\
\text { transition diagram }\end{array}$ & 7.2.3.3.7 \\
\hline 3 & $\begin{array}{c}\text { T0 + } \\
\mathrm{t} \_\mathrm{C} 1 \\
(\text { with } \\
t \_C 1> \\
\left.t \_B 1\right)\end{array}$ & $\begin{array}{c}\text { UE C T230 } \\
\text { expires }\end{array}$ & - & - & $\begin{array}{l}\text { O: start- } \\
\text { stop }\end{array}$ & $\begin{array}{l}\text { UE } \mathbf{C} \text { terminates } \\
\text { the floor } \\
\text { participant state } \\
\text { transition diagram }\end{array}$ & 7.2.3.3.7 \\
\hline 4 & $\begin{array}{l}\text { T0 + } \\
\text { t_C2 } \\
(\text { with } \\
t \_C 2> \\
\left.t \_C 1\right)\end{array}$ & $\begin{array}{c}\text { UE C } \\
\text { presses } \\
\text { PTT button }\end{array}$ & - & - & $\begin{array}{c}\text { O: } \\
\text { pending } \\
\text { request }\end{array}$ & $\begin{array}{c}\text { UE C creates an } \\
\text { instance of floor } \\
\text { participant, sends } \\
\text { Floor Request, } \\
\text { starts T201 and } \\
\text { initializes C201 }\end{array}$ & 7.2.3.2.5 \\
\hline 5 & $\begin{array}{l}\mathrm{T} 0+ \\
\mathrm{t} \_\mathrm{C} 2+ \\
\mathrm{t} \_\mathrm{CA} 1\end{array}$ & $\begin{array}{l}\text { UE A and } \\
\text { UE B } \\
\text { receive } \\
\text { Floor } \\
\text { Request }\end{array}$ & - & - & - & $\begin{array}{l}\text { UE A and UE B } \\
\text { discard the floor } \\
\text { control message }\end{array}$ & 7.2.3.1 \\
\hline
\end{tabular}




\begin{tabular}{|c|c|c|c|c|c|c|c|}
\hline & Timeline & $\begin{array}{c}\text { Triggering } \\
\text { event }\end{array}$ & $\begin{array}{l}\text { UE A state } \\
\text { transition }\end{array}$ & $\begin{array}{l}\text { UE B state } \\
\text { transition }\end{array}$ & $\begin{array}{l}\text { UE C state } \\
\text { transition }\end{array}$ & $\begin{array}{c}\text { Consequent } \\
\text { behavior }\end{array}$ & $\begin{array}{c}\text { Reference } \\
\text { for } \\
\text { details }\end{array}$ \\
\hline 6 & $\begin{array}{c}\text { T0 + } \\
\text { t_A1 } \\
\text { (with } \\
t \_A 1> \\
t \_C 2+ \\
\left.t \_C A 1\right)\end{array}$ & $\begin{array}{c}\text { UE A } \\
\text { presses } \\
\text { PTT button }\end{array}$ & $\begin{array}{c}\text { O: } \\
\text { pending } \\
\text { request }\end{array}$ & - & - & $\begin{array}{l}\text { UE A creates an } \\
\text { instance of floor } \\
\text { participant, sends } \\
\text { Floor Request, } \\
\text { starts T201 and } \\
\text { initializes C201 }\end{array}$ & 7.2.3.2.5 \\
\hline 7 & $\begin{array}{c}\mathrm{T} 0+ \\
\mathrm{t} \_\mathrm{A} 1+ \\
\mathrm{t} \_\mathrm{AB} 1\end{array}$ & $\begin{array}{l}\text { UE B and } \\
\text { UE C } \\
\text { receive } \\
\text { Floor } \\
\text { Request }\end{array}$ & - & - & - & $\begin{array}{l}\text { UE B discards the } \\
\text { floor control } \\
\text { message; } \\
\text { UE C restarts T201 } \\
\text { and resets C201 }\end{array}$ & $\begin{array}{r}\text { 7.2.3.1 } \\
7.2 .3 .6 .10\end{array}$ \\
\hline 8 & $\begin{array}{c}\text { T0 + } \\
\text { t_A1 + } \\
\text { T201 }\end{array}$ & $\begin{array}{l}\text { UE A T201 } \\
\text { expires }\end{array}$ & - & - & - & $\begin{array}{l}\text { UE A sends Floor } \\
\text { Request, starts } \\
\text { T201 and } \\
\text { increments C201 }\end{array}$ & 7.2.3.6.9 \\
\hline 9 & $\begin{array}{c}\text { T0 + } \\
\text { t_A1 + } \\
\text { T201 + } \\
\text { t_AB2 + }\end{array}$ & $\begin{array}{l}\text { UE B and } \\
\text { UE C } \\
\text { receive } \\
\text { Floor } \\
\text { Request }\end{array}$ & - & - & - & $\begin{array}{l}\text { UE B discards the } \\
\text { floor control } \\
\text { message; } \\
\text { UE C restarts T201 } \\
\text { and resets C201 }\end{array}$ & $\begin{array}{r}\text { 7.2.3.1 } \\
7.2 .3 .6 .10\end{array}$ \\
\hline 10 & $\begin{array}{c}\text { T0 + } \\
\text { t_A1 + } \\
\text { n*T201 }\end{array}$ & $\begin{array}{l}\text { UE A T201 } \\
\text { expires } \\
\text { (upper limit } \\
\text { of C201 } \\
\text { reached) }\end{array}$ & $\begin{array}{c}\text { O: has } \\
\text { permission }\end{array}$ & - & - & $\begin{array}{c}\text { UE A sends Floor } \\
\text { Taken }\end{array}$ & 7.2.3.6.6 \\
\hline 11 & $\begin{array}{c}\text { T0+ } \\
\text { t_A1 + } \\
\text { n*T201 } \\
+\mathrm{t} \_A B 3\end{array}$ & $\begin{array}{l}\text { UE B and } \\
\text { UE C } \\
\text { receive } \\
\text { Floor } \\
\text { Taken }\end{array}$ & - & $\begin{array}{l}\text { O: has no } \\
\text { permission }\end{array}$ & - & $\begin{array}{c}\text { UE B creates an } \\
\text { instance of floor } \\
\text { participant, sets } \\
\text { SSRC of current } \\
\text { floor arbitrator to } \\
\text { SSRC of UE A, } \\
\text { and starts T203; } \\
\text { UE C sets SSRC of } \\
\text { current floor } \\
\text { arbitrator to SSRC } \\
\text { of UE A, restarts } \\
\text { T201 and resets } \\
\text { C201 }\end{array}$ & $\begin{array}{r}7.2 .3 .2 .6 \\
7.2 .3 .6 .11\end{array}$ \\
\hline
\end{tabular}




\begin{tabular}{|c|c|c|c|c|c|c|c|}
\hline & Timeline & $\begin{array}{c}\text { Triggering } \\
\text { event }\end{array}$ & $\begin{array}{l}\text { UE A state } \\
\text { transition }\end{array}$ & $\begin{array}{l}\text { UE B state } \\
\text { transition }\end{array}$ & $\begin{array}{l}\text { UE C state } \\
\text { transition }\end{array}$ & $\begin{array}{c}\text { Consequent } \\
\text { behavior }\end{array}$ & $\begin{array}{c}\begin{array}{c}\text { Reference } \\
\text { for } \\
\text { details }\end{array} \\
\end{array}$ \\
\hline 12 & $\begin{array}{c}\text { T0 + } \\
\text { t_A1 + } \\
\text { n*T201 } \\
+ \text { t_AB3 } \\
+ \text { T201 }\end{array}$ & $\begin{array}{l}\text { UE C T201 } \\
\text { expires }\end{array}$ & - & - & - & $\begin{array}{l}\text { UE C sends Floor } \\
\text { Request, starts } \\
\text { T201 and } \\
\text { increments C201 }\end{array}$ & 7.2.3.6.9 \\
\hline 13 & $\begin{array}{c}\text { T0 + } \\
\text { t_A1 + } \\
\text { n*T201 } \\
+ \text { t_AB3 } \\
+ \text { T201+ } \\
\text { t_CA2 }\end{array}$ & $\begin{array}{l}\text { UE A and } \\
\text { UE B } \\
\text { receive } \\
\text { Floor } \\
\text { Request }\end{array}$ & - & - & - & $\begin{array}{l}\text { UE A stores the } \\
\text { request and sends } \\
\text { Floor Queue } \\
\text { Position Info; } \\
\text { UE B discards the } \\
\text { floor control } \\
\text { message }\end{array}$ & $\begin{array}{r}7.2 .3 .5 .4 \\
7.2 .3 .1\end{array}$ \\
\hline 14 & $\begin{array}{c}\text { T0+ } \\
\text { t_A1 + } \\
\text { n*T201 } \\
+ \text { t_AB3 } \\
+ \text { T201+ } \\
\text { t_CA2 + } \\
\text { t_AB4 }\end{array}$ & $\begin{array}{l}\text { UE B and } \\
\text { UE C } \\
\text { receive } \\
\text { Floor } \\
\text { Queue } \\
\text { Position } \\
\text { Info }\end{array}$ & - & - & O: queued & $\begin{array}{l}\text { UE B discards the } \\
\text { floor control } \\
\text { message; } \\
\text { UE C updates the } \\
\text { queue status, and } \\
\text { stops T201 }\end{array}$ & $\begin{array}{r}7.2 .3 .1 \\
7.2 .3 .6 .3\end{array}$ \\
\hline 15 & $\begin{array}{c}\text { T0 + } \\
\text { t_A2 } \\
\text { (with } \\
t \_A 2> \\
t \_A 1+ \\
n * T 201 \\
+t \_A B 3 \\
+T 201+ \\
t \_C A 2+ \\
\left.t \_A B 4\right) \\
\end{array}$ & $\begin{array}{c}\text { UE A } \\
\text { sends RTP } \\
\text { media }\end{array}$ & - & - & - & UE A starts T206 & 7.2.3.5.2 \\
\hline 16 & $\begin{array}{c}\text { T0 + } \\
\text { t_A2 + } \\
\text { t_AB5 }\end{array}$ & $\begin{array}{l}\text { UE B and } \\
\text { UE C } \\
\text { receive } \\
\text { RTP media }\end{array}$ & - & - & - & $\begin{array}{c}\text { UE B renders RTP } \\
\text { packets, starts } \\
\text { T203; } \\
\text { UE C renders RTP } \\
\text { media, and starts } \\
\text { T203 }\end{array}$ & $\begin{array}{l}7.2 .3 .4 .6 \\
7.2 .3 .8 .2\end{array}$ \\
\hline 17 & $\begin{array}{c}\mathrm{T} 0+ \\
\mathrm{t} \_\mathrm{C} 3 \\
(\text { with } \\
t \_C 3> \\
t \_A 2+ \\
\left.t_{-} A B 5\right)\end{array}$ & $\begin{array}{l}\text { UE C } \\
\text { requests the } \\
\text { queue } \\
\text { position } \\
\text { information }\end{array}$ & - & - & - & $\begin{array}{c}\text { UE C sends Floor } \\
\text { Queue Position } \\
\text { Request, starts } \\
\text { T204 and } \\
\text { initializes C204 }\end{array}$ & 7.2.3.8.11 \\
\hline
\end{tabular}




\begin{tabular}{|c|c|c|c|c|c|c|c|}
\hline & Timeline & $\begin{array}{c}\text { Triggering } \\
\text { event }\end{array}$ & $\begin{array}{l}\text { UE A state } \\
\text { transition }\end{array}$ & $\begin{array}{l}\text { UE B state } \\
\text { transition }\end{array}$ & $\begin{array}{l}\text { UE C state } \\
\text { transition }\end{array}$ & $\begin{array}{c}\text { Consequent } \\
\text { behavior }\end{array}$ & $\begin{array}{c}\text { Reference } \\
\text { for } \\
\text { details } \\
\end{array}$ \\
\hline 18 & $\begin{array}{c}\mathrm{T} 0+ \\
\text { t_C3 + } \\
\text { t_CA3 }\end{array}$ & $\begin{array}{l}\text { UE A and } \\
\text { UE B } \\
\text { receive } \\
\text { Floor } \\
\text { Queue } \\
\text { Position } \\
\text { Request }\end{array}$ & - & - & - & $\begin{array}{l}\text { UE A sends Floor } \\
\text { Queue Position } \\
\text { Info; } \\
\text { UE B discards the } \\
\text { floor control } \\
\text { message }\end{array}$ & $\begin{array}{r}7.2 .3 .5 .8 \\
7.2 .3 .1\end{array}$ \\
\hline 19 & $\begin{array}{c}\mathrm{T} 0+ \\
\mathrm{t} \_\mathrm{C} 3+ \\
\mathrm{t} \_\mathrm{CA} 3+ \\
\mathrm{t} \_\mathrm{AB} 6\end{array}$ & $\begin{array}{l}\text { UE B and } \\
\text { UE C } \\
\text { receive } \\
\text { Floor } \\
\text { Queue } \\
\text { Position } \\
\text { Info } \\
\end{array}$ & - & - & - & $\begin{array}{l}\text { UE B discards the } \\
\text { floor control } \\
\text { message; } \\
\text { UE } \mathbf{C} \text { updates the } \\
\text { queue position, and } \\
\text { stops T204 }\end{array}$ & $\begin{array}{r}7.2 .3 .1 \\
7.2 .3 .8 .3\end{array}$ \\
\hline 20 & $\begin{array}{c}\text { T0 + } \\
\text { t_A3 } \\
(\text { with } \\
t \_A 3> \\
t \_C 3+ \\
t \_C A 3+ \\
\left.t \_A B 6\right)\end{array}$ & $\begin{array}{l}\text { UE A stops } \\
\text { sending } \\
\text { RTP media }\end{array}$ & $\begin{array}{c}\text { O: } \\
\text { pending } \\
\text { granted }\end{array}$ & - & - & $\begin{array}{l}\text { UE A stops T206 } \\
\text { and T207, sends } \\
\text { Floor Granted to } \\
\text { UE C, sets stored } \\
\text { SSRC of current } \\
\text { floor arbitrator to } \\
\text { SSRC of UE C, } \\
\text { starts T205 and } \\
\text { initializes C205 }\end{array}$ & 7.2.3.5.6 \\
\hline 21 & $\begin{array}{l}\text { T0 + } \\
\text { t_A3 + } \\
\text { t_AB7 }\end{array}$ & $\begin{array}{l}\text { UE B and } \\
\text { UE C } \\
\text { receive } \\
\text { Floor } \\
\text { Granted }\end{array}$ & - & - & - & $\begin{array}{c}\text { UE B stops } \\
\text { rendering RTP } \\
\text { packets, sets stored } \\
\text { SSRC of candidate } \\
\text { floor arbitrator to } \\
\text { SSRC of UE C, } \\
\text { and starts T203; } \\
\text { UE C stops } \\
\text { rendering RTP } \\
\text { packets, notifies } \\
\text { the user about the } \\
\text { floor grant, and } \\
\text { starts T233 } \\
\end{array}$ & $\begin{array}{l}7.2 .3 .4 .5 \\
7.2 .3 .8 .6\end{array}$ \\
\hline 22 & $\begin{array}{c}\text { T0 + } \\
\text { t_A3 + } \\
\text { t_AB7 + } \\
\text { T233 }\end{array}$ & $\begin{array}{c}\text { UE C T233 } \\
\text { expires }\end{array}$ & - & - & O: silence & UE C starts T230 & 7.2.3.8.7 \\
\hline
\end{tabular}

And the corresponding figure is Fig. 3: 


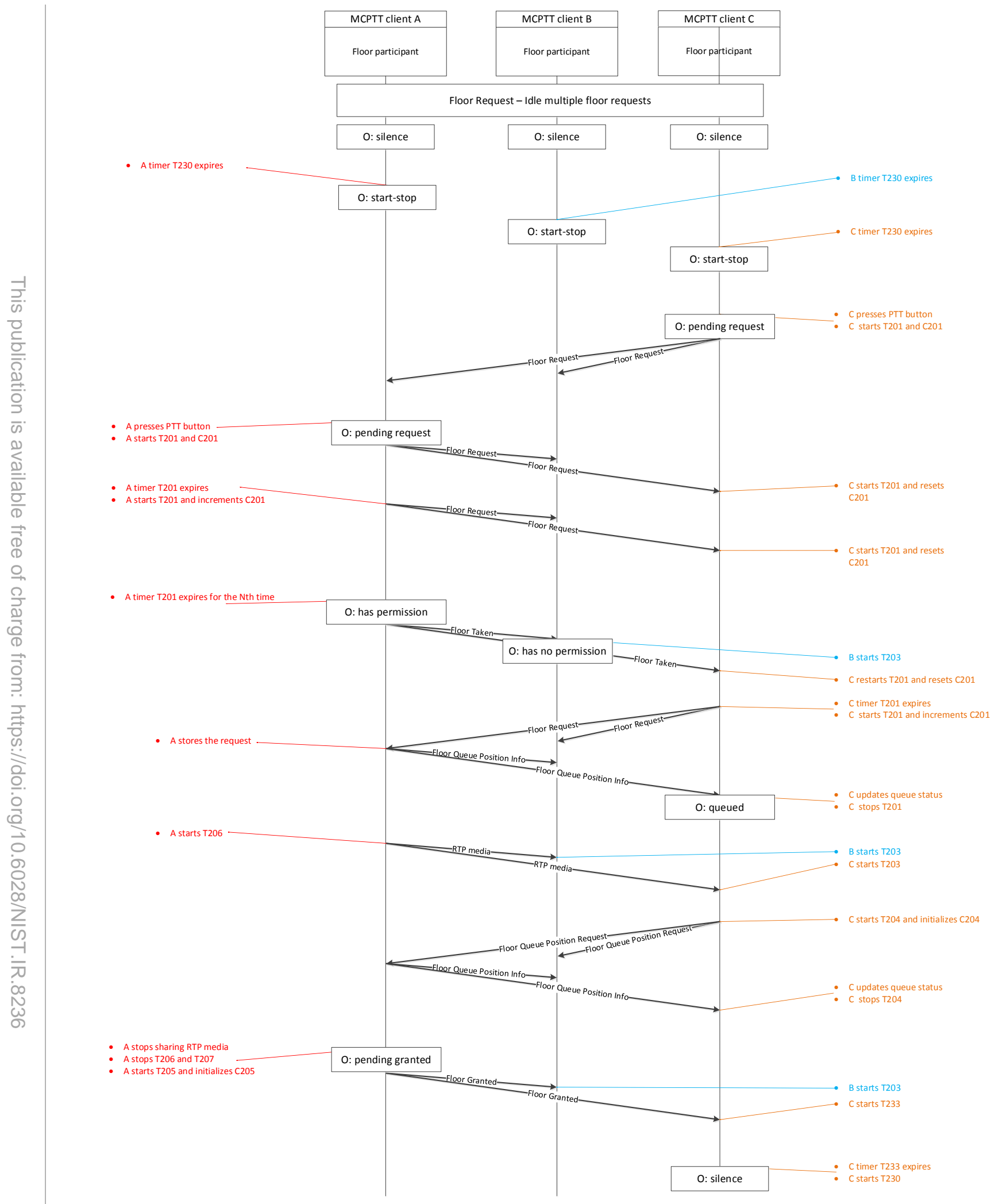

Figure 3: Floor request - idle multiple floor requests 


\subsubsection{Floor request - queued}

This scenario describes another possible process of requesting the floor, with participants beginning in the ' $\mathrm{O}$ : has permission' and the ' $\mathrm{O}$ : has no permission' state. Queuing is supported and used in this scenario: a floor participant will ask for the floor, wait in queue and finally be granted the floor. For the first few steps of this scenario, it is assumed that UE B does not receive the various floor request messages until a later time $\left(\mathrm{T} 0+4 * \mathrm{~T} 201+\mathrm{t} \_\mathrm{AB} 5\right)$. In this scenario, in addition to the multiple transmissions and receptions of floor request messages, several timer expirations (timers T201 and T205) are observed.

Scenario Purpose: To observe that a floor request is properly handled by the floor arbitrator. That is the floor request is queued and later the floor is granted.

Table 5: Floor request - queued

\begin{tabular}{|c|c|c|c|c|c|c|c|}
\hline & Timeline & $\begin{array}{c}\text { Triggering } \\
\text { event }\end{array}$ & $\begin{array}{l}\text { UE A state } \\
\text { transition }\end{array}$ & $\begin{array}{l}\text { UE B state } \\
\text { transition }\end{array}$ & $\begin{array}{l}\text { UE C state } \\
\text { transition }\end{array}$ & $\begin{array}{c}\text { Consequent } \\
\text { behavior }\end{array}$ & $\begin{array}{c}\text { Reference } \\
\text { for } \\
\text { details }\end{array}$ \\
\hline 0 & - & - & $\begin{array}{c}\text { O: has no } \\
\text { permission }\end{array}$ & $\begin{array}{c}\text { O: has } \\
\text { permission }\end{array}$ & $\begin{array}{c}\text { O: has no } \\
\text { permission }\end{array}$ & - & - \\
\hline 1 & T0 & $\begin{array}{c}\text { UE A } \\
\text { pushes PTT } \\
\text { button }\end{array}$ & $\begin{array}{c}\text { O: } \\
\text { pending } \\
\text { request }\end{array}$ & - & - & $\begin{array}{c}\text { UE A sends Floor } \\
\text { Request, starts } \\
\text { T201 and initializes } \\
\text { C201 }\end{array}$ & 7.2.3.4.2 \\
\hline 2 & $\begin{array}{l}\text { T0 + } \\
\text { T201 }\end{array}$ & $\begin{array}{c}\text { UE A T201 } \\
\text { expires }\end{array}$ & - & - & - & $\begin{array}{c}\text { UE A resends } \\
\text { Floor Request, } \\
\text { starts T201 and } \\
\text { increments C201 }\end{array}$ & 7.2.3.6.9 \\
\hline 3 & $\begin{array}{c}\mathrm{T} 0+ \\
2 * \mathrm{~T} 201\end{array}$ & $\begin{array}{c}\text { UE A T201 } \\
\text { expires }\end{array}$ & - & - & - & $\begin{array}{c}\text { UE A resends } \\
\text { Floor Request, } \\
\text { starts T201 and } \\
\text { increments C201 }\end{array}$ & 7.2.3.6.9 \\
\hline 4 & $\begin{array}{c}\text { T0+ } \\
\text { t_B1 } \\
(\text { with } \\
t \_B 1> \\
2 * T 201)\end{array}$ & $\begin{array}{c}\text { UE B } \\
\text { sends RTP } \\
\text { packets }\end{array}$ & - & - & - & UE B starts T206 & 7.2.3.5.2 \\
\hline 5 & $\begin{array}{l}\text { T0 + } \\
\text { t_B1 + } \\
\text { t_BA1 }\end{array}$ & $\begin{array}{c}\text { UE A and } \\
\text { UE C } \\
\text { receive } \\
\text { RTP } \\
\text { packets }\end{array}$ & - & - & - & $\begin{array}{c}\text { UE A restarts } \\
\text { T203, and resets } \\
\text { C201; } \\
\text { UE C restarts T203 }\end{array}$ & $\begin{array}{l}\text { 7.2.3.6.2 } \\
\text { 7.2.3.4.6 }\end{array}$ \\
\hline 6 & $\begin{array}{c}\text { T0 + } \\
\text { 3*T201 }\end{array}$ & $\begin{array}{c}\text { UE A T201 } \\
\text { expires }\end{array}$ & - & - & - & $\begin{array}{c}\text { UE A sends Floor } \\
\text { Request, and starts } \\
\text { T201 and } \\
\text { increments C201 }\end{array}$ & 7.2.3.6.9 \\
\hline
\end{tabular}




\begin{tabular}{|c|c|c|c|c|c|c|c|}
\hline & Timeline & $\begin{array}{c}\text { Triggering } \\
\text { event }\end{array}$ & $\begin{array}{l}\text { UE A state } \\
\text { transition }\end{array}$ & $\begin{array}{l}\text { UE B state } \\
\text { transition }\end{array}$ & $\begin{array}{l}\text { UE C state } \\
\text { transition }\end{array}$ & $\begin{array}{c}\text { Consequent } \\
\text { behavior }\end{array}$ & $\begin{array}{c}\text { Reference } \\
\text { for } \\
\text { details }\end{array}$ \\
\hline 7 & $\begin{array}{c}\mathrm{T} 0+ \\
4 * \mathrm{~T} 201\end{array}$ & $\begin{array}{c}\text { UE A T201 } \\
\text { expires }\end{array}$ & - & - & - & $\begin{array}{l}\text { UE A sends Floor } \\
\text { Request, starts } \\
\text { T201 and } \\
\text { increments C201 }\end{array}$ & 7.2.3.6.9 \\
\hline 8 & $\begin{array}{c}\text { T0+ } \\
4 * \mathrm{~T} 201 \\
+\mathrm{t} \_\mathrm{AB} 1\end{array}$ & $\begin{array}{c}\text { UE B } \\
\text { receives } \\
\text { Floor } \\
\text { Request } \\
\end{array}$ & - & - & - & $\begin{array}{l}\text { UE B stores the } \\
\text { request, and sends } \\
\text { Floor Queue } \\
\text { Position Info }\end{array}$ & 7.2.3.5.4 \\
\hline 9 & $\begin{array}{c}\text { T0+ } \\
4 * \mathrm{~T} 201 \\
+ \text { t_AB1 } \\
+ \text { t_BA2 }\end{array}$ & $\begin{array}{c}\text { UE A and } \\
\text { UE C } \\
\text { receive } \\
\text { Floor } \\
\text { Queue } \\
\text { Position } \\
\text { Info } \\
\end{array}$ & O: queued & - & - & $\begin{array}{l}\text { UE A updates the } \\
\text { queue status and } \\
\text { stops T201 }\end{array}$ & 7.2.3.6.3 \\
\hline 10 & $\begin{array}{c}\text { T0+ } \\
\text { t_B2 } \\
\text { (with }+ \\
4 * T 201 \\
+t \_A B 1 \\
\left.+t \_B A 2\right)\end{array}$ & $\begin{array}{c}\text { UE B } \\
\text { sends RTP } \\
\text { packets }\end{array}$ & - & - & - & UE B starts T206 & 7.2.3.5.2 \\
\hline 11 & $\begin{array}{l}\text { T0 + } \\
\text { t_B2 + } \\
\text { t_BA3 }\end{array}$ & $\begin{array}{l}\text { UE A and } \\
\text { UE C } \\
\text { receive } \\
\text { RTP } \\
\text { packets } \\
\end{array}$ & - & - & - & $\begin{array}{c}\text { UE A restarts } \\
\text { T203; } \\
\text { UE C restarts T203 }\end{array}$ & $\begin{array}{l}7.2 .3 .8 .2 \\
7.2 .3 .4 .6\end{array}$ \\
\hline 12 & $\begin{array}{l}\mathrm{T} 0+ \\
\mathrm{t} \_\mathrm{B} 3 \\
\text { (with } \\
t \_B 3> \\
t \_B 2+ \\
\left.t \_B A 3\right)\end{array}$ & $\begin{array}{c}\text { UE B } \\
\text { releases } \\
\text { PTT button }\end{array}$ & - & $\begin{array}{c}\text { O: } \\
\text { pending } \\
\text { granted }\end{array}$ & - & $\begin{array}{l}\text { UE B stops sending } \\
\text { RTP packets, sends } \\
\text { Floor Granted, } \\
\text { stops T206 and } \\
\text { T207 (if running), } \\
\text { sets SSRC of } \\
\text { current arbitrator to } \\
\text { SSRC of user (UE } \\
\text { A) to whom the } \\
\text { floor was granted, } \\
\text { starts T205 and } \\
\text { initializes C205 } \\
\text { (the upper limit } \\
\text { value of C205 is 3) }\end{array}$ & 7.2.3.5.6 \\
\hline 13 & $\begin{array}{l}\text { T0+ } \\
\text { t_B3 }+ \\
\text { t_BA4 }\end{array}$ & $\begin{array}{l}\text { UE A and } \\
\text { UE C } \\
\text { receive } \\
\text { Floor } \\
\text { Granted }\end{array}$ & - & - & - & $\begin{array}{l}\text { UE A starts T233, } \\
\text { and shall notify the } \\
\text { MCPTT user of } \\
\text { Floor Grant; } \\
\text { UE C restarts T203 } \\
\text { and sets the stored } \\
\text { SSRC of the } \\
\text { current arbitrator to } \\
\text { the SSRC of UE A }\end{array}$ & $\begin{array}{l}7.2 .3 .8 .6 \\
7.2 .3 .4 .5\end{array}$ \\
\hline
\end{tabular}




\begin{tabular}{|c|c|c|c|c|c|c|c|}
\hline & Timeline & $\begin{array}{c}\text { Triggering } \\
\text { event }\end{array}$ & $\begin{array}{l}\text { UE A state } \\
\text { transition }\end{array}$ & $\begin{array}{l}\text { UE B state } \\
\text { transition }\end{array}$ & $\begin{array}{l}\text { UE C state } \\
\text { transition }\end{array}$ & $\begin{array}{c}\text { Consequent } \\
\text { behavior }\end{array}$ & $\begin{array}{l}\text { Reference } \\
\text { for } \\
\text { details }\end{array}$ \\
\hline 14 & $\begin{array}{c}\text { T0 + } \\
\text { t_B3 + } \\
\text { T205 }\end{array}$ & $\begin{array}{l}\text { UE B T205 } \\
\text { expires }\end{array}$ & - & - & - & $\begin{array}{c}\text { UE B sends Floor } \\
\text { Granted, restarts } \\
\text { T205 and } \\
\text { increments C205 } \\
\end{array}$ & 7.2.3.7.3 \\
\hline 15 & $\begin{array}{l}\text { T0+ } \\
\text { t_B3+ } \\
\text { T205+ } \\
\text { t_BA5 }\end{array}$ & $\begin{array}{l}\text { UE A and } \\
\text { UE C } \\
\text { receive } \\
\text { Floor } \\
\text { Granted for } \\
\text { the 2nd } \\
\text { time }\end{array}$ & - & - & - & $\begin{array}{l}\text { UE A shall notify } \\
\text { the MCPTT user of } \\
\text { Floor Grant; } \\
\text { UE C restarts T203 }\end{array}$ & $\begin{array}{l}\text { 7.2.3.8.6 } \\
\text { 7.2.3.4.5 }\end{array}$ \\
\hline 16 & $\begin{array}{c}\text { T0 + } \\
\text { t_B3 + } \\
\text { 2*T205 }\end{array}$ & $\begin{array}{l}\text { UE B T205 } \\
\text { expires (for } \\
\text { the second } \\
\text { time) }\end{array}$ & - & - & - & $\begin{array}{l}\text { UE B sends Floor } \\
\text { Granted, restarts } \\
\text { T205 and } \\
\text { increments C205 }\end{array}$ & 7.2.3.7.3 \\
\hline 17 & $\begin{array}{c}\text { T0+ } \\
\text { t_B3+ } \\
\text { 2*T205 } \\
+ \text { t_BA6 }\end{array}$ & $\begin{array}{l}\text { UE A and } \\
\text { UE C } \\
\text { receive } \\
\text { Floor } \\
\text { Granted }\end{array}$ & - & - & - & $\begin{array}{l}\text { UE A shall notify } \\
\text { the MCPTT user of } \\
\text { Floor Grant; } \\
\text { UE C restarts T203 }\end{array}$ & $\begin{array}{l}7.2 .3 .8 .6 \\
7.2 .3 .4 .5\end{array}$ \\
\hline 18 & $\begin{array}{c}\text { T0 + } \\
\text { t_B3 + } \\
\text { 3*T205 }\end{array}$ & $\begin{array}{l}\text { UE B T205 } \\
\text { expires (the } \\
\text { upper limit } \\
\text { value of } \\
\text { C205 is 3) }\end{array}$ & - & - & - & $\begin{array}{l}\text { UE } \mathbf{B} \text { starts T233, } \\
\text { resets C205 }\end{array}$ & 7.2.3.7.4 \\
\hline 19 & $\begin{array}{c}\text { T0+ } \\
\text { t_A1 } \\
(\text { with } \\
t \_A 1> \\
t \_B 3+ \\
2 * T 205 \\
\left.+t \_B A 6\right) \\
\end{array}$ & $\begin{array}{c}\text { UE A } \\
\text { pushes PTT } \\
\text { button }\end{array}$ & $\begin{array}{c}\text { O: has } \\
\text { permission }\end{array}$ & - & - & UE A stops T233 & 7.2.3.8.8 \\
\hline 20 & $\begin{array}{c}\mathrm{T} 0+ \\
\mathrm{t} \_\mathrm{A} 2 \\
(\text { with } \\
t \_A 2> \\
\left.t \_A 1\right)\end{array}$ & $\begin{array}{c}\text { UE A } \\
\text { sends RTP } \\
\text { packets }\end{array}$ & - & - & - & UE A starts T206 & 7.2.3.5.2 \\
\hline 21 & $\begin{array}{l}\mathrm{T} 0+ \\
\mathrm{t} \_\mathrm{A} 2+ \\
\mathrm{t} \_\mathrm{AB} 2\end{array}$ & $\begin{array}{l}\text { UE B and } \\
\text { UE C } \\
\text { receive } \\
\text { RTP } \\
\text { packets }\end{array}$ & - & $\begin{array}{l}\text { O: has no } \\
\text { permission }\end{array}$ & - & $\begin{array}{c}\text { UE B starts T203, } \\
\text { and stops T233 and } \\
\text { T205; } \\
\text { UE C restarts T203 }\end{array}$ & $\begin{array}{l}7.2 .3 .7 .2 \\
7.2 .3 .4 .6\end{array}$ \\
\hline
\end{tabular}

And the corresponding figure is Fig. 4: 


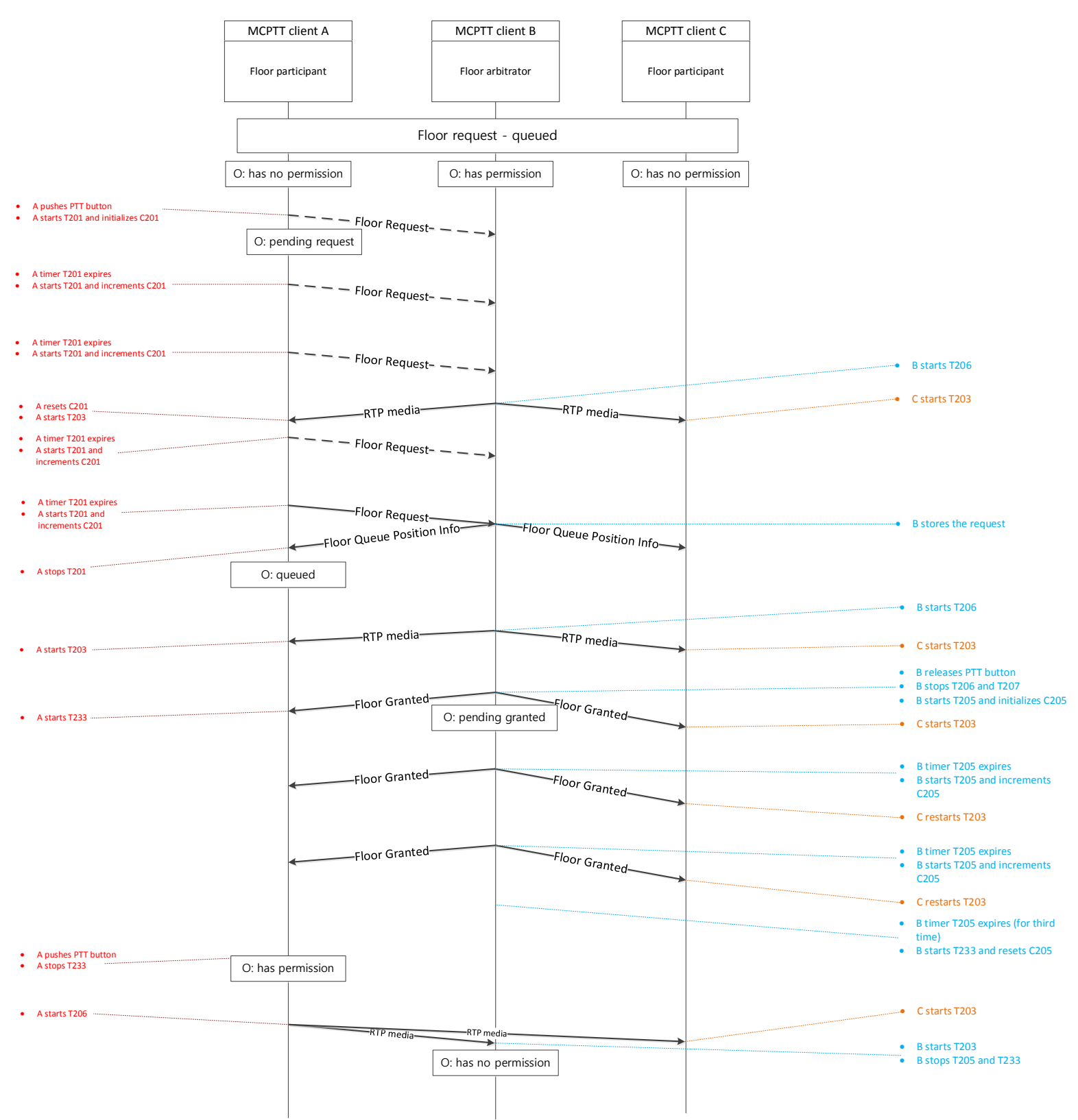

Figure 4: Floor request - queued

\subsubsection{Floor request - denied}

This scenario describes yet another possible outcome of requesting the floor, with participants beginning in the ' $\mathrm{O}$ : has permission' and the ' $\mathrm{O}$ : has no permission' state. Queuing is not supported here or the queue is already full or that the Floor Request message does not contain information that queuing is supported, so the floor participant asking for the floor is denied permission to take the floor and because the floor request is not pre-emptive.

Scenario Purpose: To observe that the floor request from UE A is denied by the floor arbitrator and UE A makes no further floor requests. 
Table 6: Floor request - denied

\begin{tabular}{|c|c|c|c|c|c|c|c|}
\hline & Timeline & $\begin{array}{c}\text { Triggering } \\
\text { event }\end{array}$ & $\begin{array}{l}\text { UE A state } \\
\text { transition }\end{array}$ & $\begin{array}{l}\text { UE B state } \\
\text { transition }\end{array}$ & $\begin{array}{l}\text { UE C state } \\
\text { transition }\end{array}$ & $\begin{array}{c}\begin{array}{c}\text { Consequent } \\
\text { behavior }\end{array} \\
\end{array}$ & $\begin{array}{l}\text { Reference } \\
\text { for details }\end{array}$ \\
\hline 0 & - & - & $\begin{array}{l}\text { O: has no } \\
\text { permission }\end{array}$ & $\begin{array}{c}\text { O: has } \\
\text { permission }\end{array}$ & $\begin{array}{c}\text { O: has no } \\
\text { permission }\end{array}$ & - & - \\
\hline 1 & T0 & $\begin{array}{c}\text { UE A } \\
\text { pushes PTT } \\
\text { button }\end{array}$ & $\begin{array}{l}\text { O: pending } \\
\text { request }\end{array}$ & - & - & $\begin{array}{c}\text { UE A sends } \\
\text { Floor Request, } \\
\text { starts T201 } \\
\text { and initializes } \\
\text { C201 }\end{array}$ & 7.2.3.4.2 \\
\hline 2 & $\begin{array}{c}\mathrm{T} 0+ \\
\mathrm{t} \_\mathrm{AB} 1\end{array}$ & $\begin{array}{l}\text { UE B and } \\
\text { UE C } \\
\text { receive } \\
\text { Floor } \\
\text { Request }\end{array}$ & - & - & - & $\begin{array}{l}\text { UE B analyzes } \\
\text { Floor Request, } \\
\text { generates and } \\
\text { sends Floor } \\
\text { Deny; } \\
\text { UE C discards } \\
\text { the floor } \\
\text { control } \\
\text { message }\end{array}$ & $\begin{array}{r}7.2 .3 .5 .4 \\
7.2 .3 .1\end{array}$ \\
\hline 3 & $\begin{array}{c}\mathrm{T} 0+ \\
\mathrm{t} \_\mathrm{AB} 1+ \\
\mathrm{t} \_\mathrm{BA} 1\end{array}$ & $\begin{array}{l}\text { UE A and } \\
\text { UE C } \\
\text { receive } \\
\text { Floor Deny }\end{array}$ & $\begin{array}{l}\text { O: has no } \\
\text { permission }\end{array}$ & - & - & $\begin{array}{l}\text { UE A provides } \\
\text { Floor Deny } \\
\text { notification to } \\
\text { the user, stops } \\
\text { T201, and } \\
\text { starts T203; } \\
\text { UE C discards } \\
\text { the floor } \\
\text { control } \\
\text { message }\end{array}$ & $\begin{array}{r}7.2 .3 .6 .4 \\
7.2 .3 .1\end{array}$ \\
\hline
\end{tabular}

And the corresponding figure is Fig. 5:

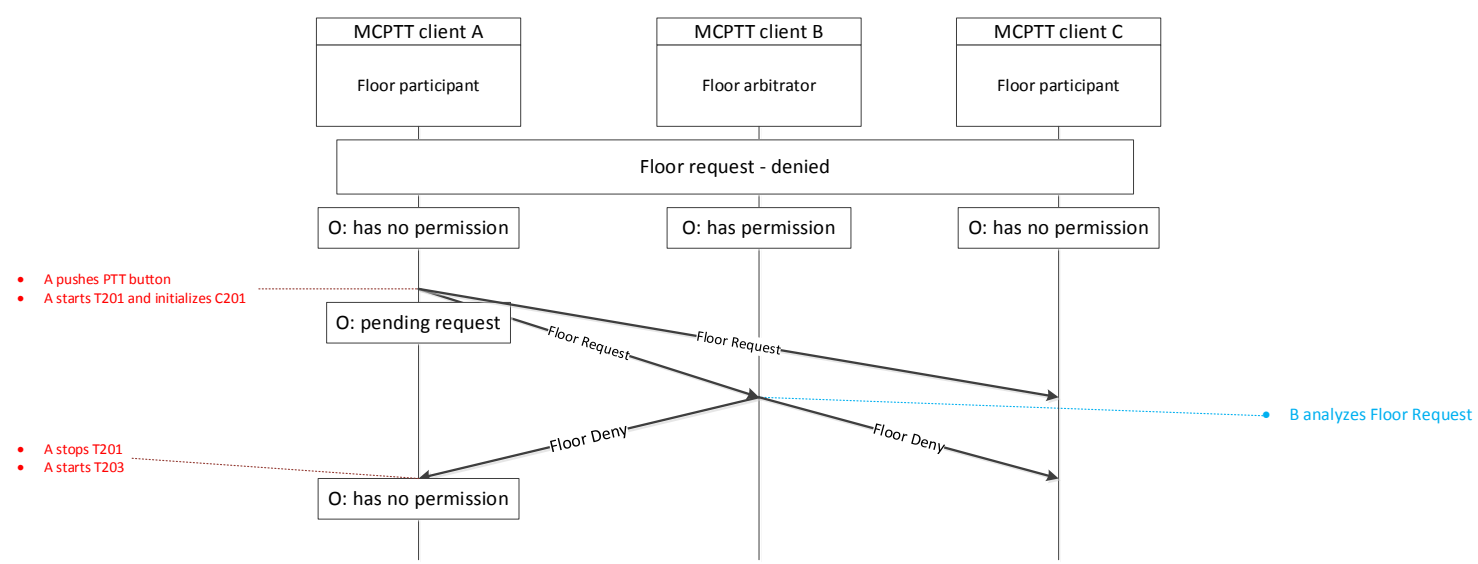

Figure 5: Floor request - denied

\subsubsection{Floor request - pre-emptive}

This scenario describes the process of requesting the floor, when the user requesting the floor has a higher floor priority than the current speaker. 
Scenario Purpose: To observe that the current floor arbitrator relinquishes the floor when another user requests the floor with a higher floor priority (pre-emptive).

Table 7: Floor request - pre-emptive

\begin{tabular}{|c|c|c|c|c|c|c|c|}
\hline & Timeline & $\begin{array}{c}\text { Triggering } \\
\text { event }\end{array}$ & $\begin{array}{l}\text { UE A state } \\
\text { transition }\end{array}$ & $\begin{array}{l}\text { UE B state } \\
\text { transition }\end{array}$ & $\begin{array}{c}\text { UE C state } \\
\text { transition }\end{array}$ & $\begin{array}{c}\text { Consequent } \\
\text { behavior }\end{array}$ & $\begin{array}{c}\text { Reference } \\
\text { for } \\
\text { details } \\
\end{array}$ \\
\hline 0 & & & $\begin{array}{c}\text { O: has no } \\
\text { permission }\end{array}$ & $\begin{array}{c}\text { O: has } \\
\text { permission }\end{array}$ & $\begin{array}{c}\text { O: has no } \\
\text { permission }\end{array}$ & & \\
\hline 1 & T0 & $\begin{array}{c}\text { UE B } \\
\text { sends RTP } \\
\text { packet } \\
\end{array}$ & - & - & - & $\begin{array}{c}\text { UE B starts } \\
\text { T206 }\end{array}$ & 7.2.3.5.2 \\
\hline 2 & $\begin{array}{l}\text { T0 + } \\
\text { t_BA1 }\end{array}$ & $\begin{array}{l}\text { UE A and } \\
\text { UE C } \\
\text { receive } \\
\text { RTP } \\
\text { packets } \\
\end{array}$ & - & - & - & $\begin{array}{c}\text { UE A and UE C } \\
\text { start T203 }\end{array}$ & 7.2.3.4.6 \\
\hline 3 & $\begin{array}{l}\text { T0 + } \\
\text { t_B1 }\end{array}$ & $\begin{array}{c}\text { UE B } \\
\text { sends RTP } \\
\text { packet }\end{array}$ & - & - & - & $\begin{array}{c}\text { UE B starts } \\
\text { T206 }\end{array}$ & 7.2.3.5.2 \\
\hline 4 & $\begin{array}{c}\text { T0 + } \\
\text { t_B1 + } \\
\text { t_BA2 }\end{array}$ & $\begin{array}{l}\text { UE A and } \\
\text { UE C } \\
\text { receive } \\
\text { RTP packet }\end{array}$ & - & - & - & $\begin{array}{c}\text { UE A and UE C } \\
\text { start T203 }\end{array}$ & 7.2.3.4.6 \\
\hline 5 & $\begin{array}{c}\mathrm{T} 0+ \\
\mathrm{t} \_\mathrm{A} 1 \\
(\text { with } \\
t \_A 1> \\
t \_B 1+ \\
\left.t \_B A 2\right)\end{array}$ & $\begin{array}{c}\text { UE A } \\
\text { receives } \\
\text { indication } \\
\text { from } \\
\text { MCPTT } \\
\text { user that } \\
\text { the user } \\
\text { wants to } \\
\text { send media }\end{array}$ & $\begin{array}{l}\text { O: pending } \\
\text { request }\end{array}$ & - & - & $\begin{array}{l}\text { UE A sends } \\
\text { Floor Request, } \\
\text { starts T201 and } \\
\text { initializes C201 }\end{array}$ & 7.2.3.4.2 \\
\hline 6 & $\begin{array}{l}\text { T0 + } \\
\text { t_A1 + } \\
\text { t_AB1 }\end{array}$ & $\begin{array}{l}\text { UE B and } \\
\text { UE C } \\
\text { receive } \\
\text { Floor } \\
\text { Request }\end{array}$ & - & $\begin{array}{c}\text { O: } \\
\text { pending } \\
\text { granted }\end{array}$ & - & $\begin{array}{c}\text { UE B } \\
\text { determines } \\
\text { UE A's pre- } \\
\text { emption priority } \\
\text { is higher than its } \\
\text { own priority, } \\
\text { stops sending } \\
\text { RTP packets, } \\
\text { stops T206 and } \\
\text { T207(if } \\
\text { running), sends } \\
\text { Floor Granted, } \\
\text { starts T205 and } \\
\text { initializes C205; } \\
\text { UE C discards } \\
\text { the floor control } \\
\text { message }\end{array}$ & $\begin{array}{r}\text { 7.2.3.5.7 } \\
7.2 .3 .1\end{array}$ \\
\hline
\end{tabular}




\begin{tabular}{|c|c|c|c|c|c|c|c|}
\hline & Timeline & $\begin{array}{c}\text { Triggering } \\
\text { event }\end{array}$ & $\begin{array}{l}\text { UE A state } \\
\text { transition }\end{array}$ & $\begin{array}{l}\text { UE B state } \\
\text { transition }\end{array}$ & $\begin{array}{l}\text { UE C state } \\
\text { transition }\end{array}$ & $\begin{array}{c}\text { Consequent } \\
\text { behavior }\end{array}$ & $\begin{array}{c}\text { Reference } \\
\text { for } \\
\text { details }\end{array}$ \\
\hline 7 & $\begin{array}{c}\text { T0 + } \\
\text { t_A1 + } \\
\text { t_AB1 + } \\
\text { t_BA3 }\end{array}$ & $\begin{array}{l}\text { UE A and } \\
\text { UE C } \\
\text { receive } \\
\text { Floor } \\
\text { Granted }\end{array}$ & $\begin{array}{c}\text { O: has } \\
\text { permission }\end{array}$ & - & - & $\begin{array}{l}\text { UE A stops } \\
\text { rendering RTP } \\
\text { packets, stops } \\
\text { T201 and T203, } \\
\text { and may provide } \\
\text { floor granted } \\
\text { notification to } \\
\text { MCPTT user; } \\
\text { UE C stops } \\
\text { rendering RTP } \\
\text { packets, restarts } \\
\text { T203, sets the } \\
\text { stored SSRC of } \\
\text { candidate } \\
\text { arbitrator to the } \\
\text { SSRC of UE A, } \\
\text { and may provide } \\
\text { floor taken } \\
\text { notification } \\
\end{array}$ & $\begin{array}{l}\text { 7.2.3.6.7 } \\
\text { 7.2.3.4.5 }\end{array}$ \\
\hline 8 & $\begin{array}{c}\text { T0+ } \\
\mathrm{t} \_\mathrm{A} 1+ \\
\mathrm{t} \_\mathrm{AB} 1+ \\
\mathrm{T} 205\end{array}$ & $\begin{array}{c}\text { UE B T205 } \\
\text { expires }\end{array}$ & - & - & - & $\begin{array}{l}\text { UE B resends } \\
\text { Floor Granted, } \\
\text { restarts T205 } \\
\text { and increments } \\
\text { C205 }\end{array}$ & 7.2.3.7.3 \\
\hline 9 & $\begin{array}{c}\text { T0 + } \\
\text { t_A1 + } \\
\text { t_AB1 + } \\
\text { T205 + } \\
\text { t_BA4 }\end{array}$ & $\begin{array}{l}\text { UE A and } \\
\text { UE C } \\
\text { receive } \\
\text { Floor } \\
\text { Granted }\end{array}$ & - & - & - & $\begin{array}{l}\text { UE A discards } \\
\text { the floor control } \\
\text { message; } \\
\text { UE C checks if } \\
\text { SSRC of Floor } \\
\text { Participant (UE } \\
\text { B) sending the } \\
\text { Floor Granted } \\
\text { matches SSRC } \\
\text { of current } \\
\text { arbitrator (UE } \\
\text { A) }\end{array}$ & $\begin{array}{r}\text { 7.2.3.1 } \\
7.2 .3 .4 .5\end{array}$ \\
\hline 10 & $\begin{array}{c}\text { T0 + } \\
\text { t_A2 } \\
\text { (with } \\
t \_A 2> \\
t \_A 1+ \\
t \_A B 1+ \\
T 205+ \\
\left.t \_B A 4\right) \\
\end{array}$ & $\begin{array}{c}\text { UE A } \\
\text { sends RTP } \\
\text { packets }\end{array}$ & - & - & - & $\begin{array}{c}\text { UE A starts } \\
\text { T206 }\end{array}$ & 7.2.3.5.2 \\
\hline 11 & $\begin{array}{c}\text { T0 + } \\
\text { t_A2 + } \\
\text { t_AB2 }\end{array}$ & $\begin{array}{l}\text { UE B and } \\
\text { UE C } \\
\text { receive } \\
\text { RTP } \\
\text { Packets }\end{array}$ & - & $\begin{array}{c}\text { O: has no } \\
\text { permission }\end{array}$ & - & $\begin{array}{c}\text { UE B stops } \\
\text { T205 and T233, } \\
\text { and starts T203; } \\
\text { UE C starts } \\
\text { T203 }\end{array}$ & $\begin{array}{l}7.2 .3 .7 .2 \\
7.2 .3 .4 .6\end{array}$ \\
\hline
\end{tabular}


And the corresponding figure is Fig. 6:

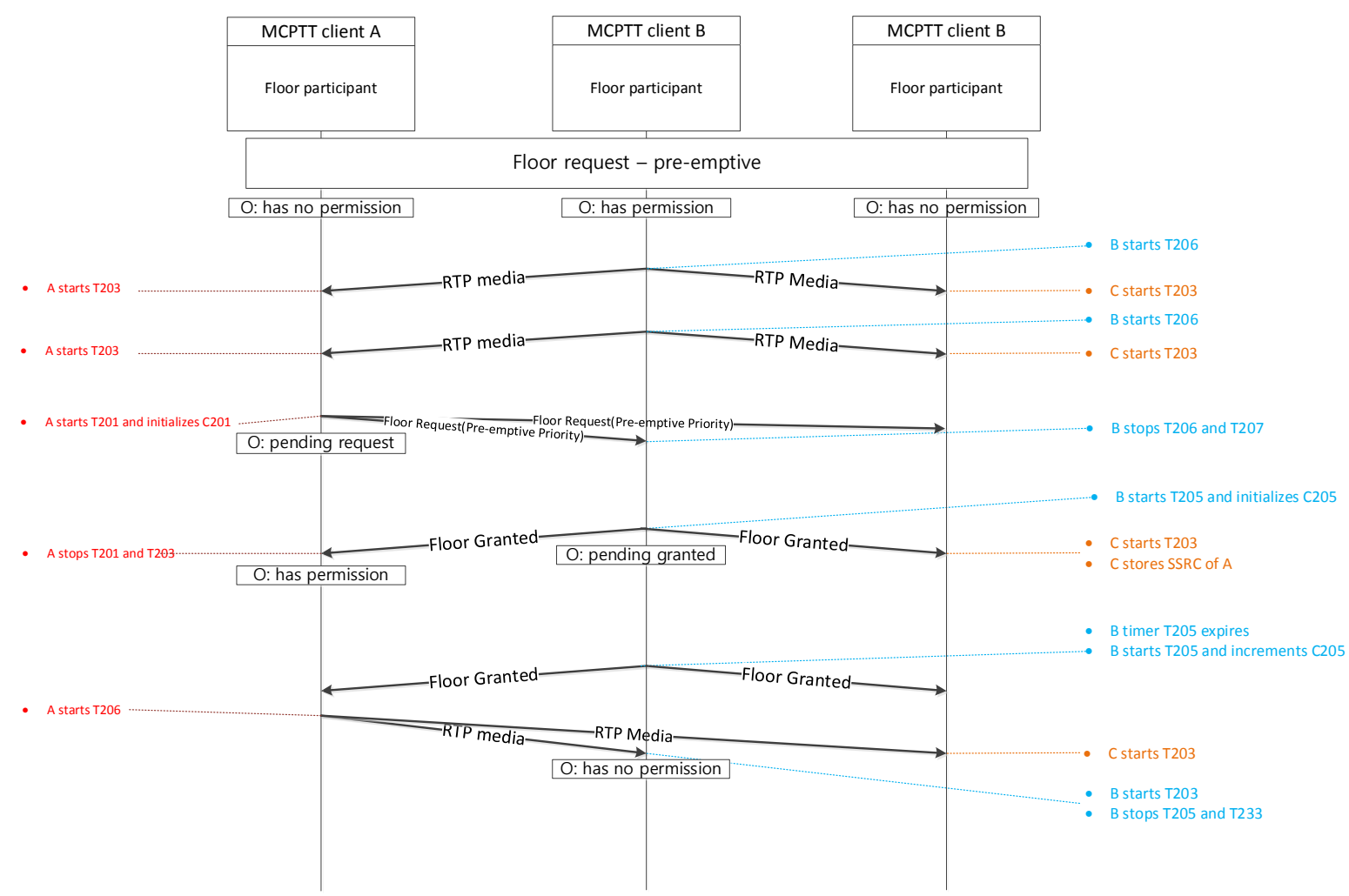

Figure 6: Floor request - pre-emptive

\subsubsection{Floor release}

\subsubsection{Floor release by the floor arbitrator}

In this scenario, one user has the permission to talk (floor arbitrator), while the other one does not. The floor arbitrator (UE A) stops talking and releases the floor and there is no other user waiting in the queue. It is assumed in this scenario that the floor participant (UE B) receives Floor Release message before the expiry of its own T203 timer.

Scenario Purpose: To observe that when the current arbitrator stops transmitting with an empty queue, that it sends a Floor Release message and that the participants go back to the " $\mathrm{O}$ : silence” state.

Table 8: Floor release by the floor arbitrator

\begin{tabular}{|c|c|c|c|c|c|c|}
\hline & Timeline & $\begin{array}{c}\text { Triggering } \\
\text { event }\end{array}$ & $\begin{array}{c}\text { UE A state } \\
\text { transition }\end{array}$ & $\begin{array}{c}\text { UE B state } \\
\text { transition }\end{array}$ & Consequent behavior & $\begin{array}{c}\text { Reference } \\
\text { for details }\end{array}$ \\
\hline 0 & - & - & $\begin{array}{c}\text { O: has } \\
\text { permission }\end{array}$ & $\begin{array}{c}\text { O: has no } \\
\text { permission }\end{array}$ & & - \\
\hline
\end{tabular}




\begin{tabular}{|c|c|c|c|c|c|c|}
\hline & Timeline & $\begin{array}{c}\text { Triggering } \\
\text { event }\end{array}$ & $\begin{array}{l}\text { UE A state } \\
\text { transition }\end{array}$ & $\begin{array}{l}\text { UE B state } \\
\text { transition }\end{array}$ & Consequent behavior & $\begin{array}{l}\text { Reference } \\
\text { for details }\end{array}$ \\
\hline 1 & T0 & $\begin{array}{c}\text { UE A } \\
\text { sends RTP } \\
\text { packet }\end{array}$ & - & - & UE A starts T206 & 7.2.3.5.2 \\
\hline 2 & $\mathrm{~T} 0+\mathrm{t} \_\mathrm{AB} 1$ & $\begin{array}{l}\mathbf{U E} \mathbf{B} \\
\text { receives } \\
\text { RTP } \\
\text { packet }\end{array}$ & - & - & UE B restarts T203 & 7.2.3.4.6 \\
\hline 3 & $\begin{array}{c}\mathrm{T} 0+\mathrm{t} \_\mathrm{A} 1 \\
\left(\text { with } t \_A 1>t \_A B 1\right)\end{array}$ & $\begin{array}{c}\text { UE A } \\
\text { receives } \\
\text { MCPTT } \\
\text { indication } \\
\text { to stop } \\
\text { sending } \\
\text { RTP } \\
\text { packets } \\
\end{array}$ & O: silence & - & $\begin{array}{l}\text { UE A sends Floor } \\
\text { Release, starts T230, } \\
\text { stops T206 and T207 (if } \\
\text { running), and clears } \\
\text { SSRC of current } \\
\text { arbitrator }\end{array}$ & 7.2.3.5.5 \\
\hline 4 & $\begin{array}{c}\mathrm{T} 0+\mathrm{t} \_\mathrm{A} 1+\mathrm{t} \_\mathrm{AB} 2 \\
\left(\text { with }\left(t \_A 1+t \_A B 2\right)\right. \\
\left.<\left(t \_A B 1+T 203\right)\right)\end{array}$ & $\begin{array}{l}\text { UE B } \\
\text { receives } \\
\text { Floor } \\
\text { Release }\end{array}$ & - & O: silence & $\begin{array}{l}\text { UE B stops rendering } \\
\text { RTP packets, stops T203 } \\
\text { and starts T230, may } \\
\text { send floor idle } \\
\text { notification to MCPTT } \\
\text { user, and clears SSRC of } \\
\text { current arbitrator }\end{array}$ & 7.2.3.4.3 \\
\hline
\end{tabular}

\subsubsection{Floor release by a queued floor participant}

In this scenario, one user has permission to talk, and another is queued. The queued user removes his request at the beginning of the test scenario.

Scenario Purpose: To observe that a queued user can cancel its floor request during a call.

Table 9: Floor release by a queued floor participant

\begin{tabular}{|c|c|c|c|c|c|c|c|}
\hline & Timeline & $\begin{array}{c}\text { Triggering } \\
\text { event }\end{array}$ & $\begin{array}{c}\text { UE A state } \\
\text { transition }\end{array}$ & $\begin{array}{c}\text { UE B state } \\
\text { transition }\end{array}$ & $\begin{array}{c}\text { UE C state } \\
\text { transition }\end{array}$ & $\begin{array}{c}\text { Consequent } \\
\text { behavior }\end{array}$ & $\begin{array}{c}\text { Reference for } \\
\text { details }\end{array}$ \\
\hline 0 & - & - & O: queued & $\begin{array}{c}\text { O: has } \\
\text { permission }\end{array}$ & $\begin{array}{c}\text { O: has no } \\
\text { permission }\end{array}$ & - & - \\
\hline
\end{tabular}




\begin{tabular}{|c|c|c|c|c|c|c|c|}
\hline & Timeline & $\begin{array}{c}\text { Triggering } \\
\text { event }\end{array}$ & $\begin{array}{l}\text { UE A state } \\
\text { transition }\end{array}$ & $\begin{array}{l}\text { UE B state } \\
\text { transition }\end{array}$ & $\begin{array}{l}\text { UE C state } \\
\text { transition }\end{array}$ & $\begin{array}{c}\text { Consequent } \\
\text { behavior }\end{array}$ & $\begin{array}{c}\begin{array}{c}\text { Reference for } \\
\text { details }\end{array} \\
\end{array}$ \\
\hline 1 & T0 & $\begin{array}{c}\text { UE A } \\
\text { receives } \\
\text { MCPTT } \\
\text { indication } \\
\text { to release } \\
\text { the pending } \\
\text { request } \\
\end{array}$ & $\begin{array}{c}\text { O: has no } \\
\text { permission }\end{array}$ & - & - & $\begin{array}{l}\text { UE A sends } \\
\text { Floor Release, } \\
\text { stops T233 (if } \\
\text { running) }\end{array}$ & 7.2.3.8.5 \\
\hline 2 & $\begin{array}{l}\text { T0 + } \\
\text { t_AB1 }\end{array}$ & $\begin{array}{l}\text { UE B and } \\
\text { UE C } \\
\text { receive } \\
\text { Floor } \\
\text { Release }\end{array}$ & - & - & - & $\begin{array}{l}\text { UE B removes } \\
\text { UE A from the } \\
\text { queue; } \\
\text { UE C discards } \\
\text { the floor } \\
\text { control } \\
\text { message }\end{array}$ & $\begin{array}{r}7.2 .3 .5 .3 \\
7.2 .3 .1\end{array}$ \\
\hline
\end{tabular}

\subsubsection{Floor release by the floor arbitrator with queued participants}

In this scenario, one user has the permission to talk (floor arbitrator), while the other ones are waiting in the queue. The first floor arbitrator stops talking, releases the floor, and grants the floor to the first floor participant in the queue. The new floor arbitrator takes the floor and talks, then stops talking for quite a while without releasing floor explicitly. After a timer (T203) expires, other floor participants assume there is no floor arbitrator.

Scenario Purpose: To observe that a floor arbitrator can release the medium and that the floor is correctly given to the next queued user, who becomes the new floor arbitrator. When the new floor arbitrator stops talking but does not release the floor explicitly, observe that the other floor participants assume that there is no longer a floor arbitrator (i.e., T203 expires).

Table 10: Floor release by the floor participant with queued participants

\begin{tabular}{|c|c|c|c|c|c|c|c|}
\hline & Timeline & $\begin{array}{c}\text { Triggering } \\
\text { event }\end{array}$ & $\begin{array}{c}\text { UE A state } \\
\text { transition }\end{array}$ & $\begin{array}{c}\text { UE B State } \\
\text { transition }\end{array}$ & $\begin{array}{c}\text { UE C State } \\
\text { transition }\end{array}$ & $\begin{array}{c}\text { Consequent } \\
\text { behavior }\end{array}$ & $\begin{array}{c}\text { Reference } \\
\text { for details }\end{array}$ \\
\hline 0 & - & - & O: queued & $\begin{array}{c}\text { O: has } \\
\text { permission }\end{array}$ & O: queued & - & - \\
\hline
\end{tabular}




\begin{tabular}{|c|c|c|c|c|c|c|c|}
\hline & Timeline & $\begin{array}{c}\text { Triggering } \\
\text { event }\end{array}$ & $\begin{array}{l}\text { UE A state } \\
\text { transition }\end{array}$ & $\begin{array}{l}\text { UE B State } \\
\text { transition }\end{array}$ & $\begin{array}{l}\text { UE C State } \\
\text { transition }\end{array}$ & $\begin{array}{c}\text { Consequent } \\
\text { behavior }\end{array}$ & $\begin{array}{l}\text { Reference } \\
\text { for details }\end{array}$ \\
\hline 1 & T0 & $\begin{array}{c}\text { UE B } \\
\text { releases } \\
\text { PTT button }\end{array}$ & - & $\begin{array}{c}\text { O: } \\
\text { pending } \\
\text { granted }\end{array}$ & - & $\begin{array}{c}\text { UE B stops } \\
\text { sending RTP } \\
\text { packets, sends } \\
\text { Floor Granted } \\
\text { (for UE A), } \\
\text { stops T206 and } \\
\text { T207 (if } \\
\text { running), sets } \\
\text { SSRC of current } \\
\text { arbitrator to } \\
\text { SSRC of user to } \\
\text { whom the floor } \\
\text { was granted (UE } \\
\text { A), starts T205 } \\
\text { and initializes } \\
\text { C205 }\end{array}$ & 7.2.3.5.6 \\
\hline 2 & $\begin{array}{l}\text { T0 + } \\
\text { t_BA1 }\end{array}$ & $\begin{array}{l}\text { UE A and } \\
\text { UE C } \\
\text { receive } \\
\text { Floor } \\
\text { Granted }\end{array}$ & - & - & - & $\begin{array}{l}\text { UE A stops } \\
\text { rendering RTP } \\
\text { packets, starts } \\
\text { T233 (if not } \\
\text { running), shall } \\
\text { notify the } \\
\text { MCPTT user of } \\
\text { Floor Grant; } \\
\text { UE C stops } \\
\text { rendering RTP } \\
\text { packets, restarts } \\
\text { T203 and stores } \\
\text { SSRC of } \\
\text { candidate } \\
\text { arbitrator to } \\
\text { SSRC of UE A } \\
\end{array}$ & $\begin{array}{l}\text { 7.2.3.8.6 } \\
\text { 7.2.3.8.9 }\end{array}$ \\
\hline 3 & $\begin{array}{l}\text { T0+ } \\
\text { t_A1 } \\
\text { (with } \\
t \_A 1> \\
\left.t \_B A 1\right) \\
\end{array}$ & $\begin{array}{c}\text { UE A } \\
\text { pushes PTT } \\
\text { button }\end{array}$ & $\begin{array}{c}\text { O: has } \\
\text { permission }\end{array}$ & - & - & $\begin{array}{c}\text { UE A stops } \\
\text { T233 }\end{array}$ & 7.2.3.8.8 \\
\hline 4 & $\begin{array}{c}\text { T0+ } \\
\text { t_A2 } \\
\text { (with } \\
t \_A 2> \\
\left.t \_A 1\right) \\
\end{array}$ & $\begin{array}{c}\text { UE A } \\
\text { sends RTP } \\
\text { packets }\end{array}$ & - & - & - & $\begin{array}{l}\text { UE A starts } \\
\text { T206 }\end{array}$ & 7.2.3.5.2 \\
\hline 5 & $\begin{array}{c}\mathrm{T} 0+ \\
\mathrm{t} \_\mathrm{A} 2+ \\
\mathrm{t} \_\mathrm{AB} 1\end{array}$ & $\begin{array}{l}\text { UE B and } \\
\text { UE C } \\
\text { receive } \\
\text { RTP } \\
\text { packets } \\
\end{array}$ & - & $\begin{array}{c}\text { O: has no } \\
\text { permission }\end{array}$ & - & $\begin{array}{c}\text { UE B stops } \\
\text { T205 and starts } \\
\text { T203; } \\
\text { UE C restarts } \\
\text { T203 } \\
\end{array}$ & $\begin{array}{l}\text { 7.2.3.7.2 } \\
\text { 7.2.3.8.2 }\end{array}$ \\
\hline
\end{tabular}




\begin{tabular}{|c|c|c|c|c|c|c|c|}
\hline & Timeline & $\begin{array}{c}\text { Triggering } \\
\text { event }\end{array}$ & $\begin{array}{l}\text { UE A state } \\
\text { transition }\end{array}$ & $\begin{array}{l}\text { UE B State } \\
\text { transition }\end{array}$ & $\begin{array}{l}\text { UE C State } \\
\text { transition }\end{array}$ & $\begin{array}{c}\text { Consequent } \\
\text { behavior }\end{array}$ & $\begin{array}{l}\text { Reference } \\
\text { for details }\end{array}$ \\
\hline 6 & $\begin{array}{c}\mathrm{T} 0+ \\
\mathrm{t} \_\mathrm{A} 2+ \\
\mathrm{t} \_\mathrm{AB} 1+ \\
\mathrm{T} 203\end{array}$ & $\begin{array}{c}\text { UE C timer } \\
\text { T203 } \\
\text { expires }\end{array}$ & - & - & $\begin{array}{l}\text { O: pending } \\
\text { request }\end{array}$ & $\begin{array}{l}\text { UE C stops } \\
\text { rendering RTP } \\
\text { packets, clears } \\
\text { SSRC of floor } \\
\text { arbitrator, sends } \\
\text { Floor Request, } \\
\text { starts T201 and } \\
\text { initializes C201 }\end{array}$ & 7.2.3.8.10 \\
\hline 7 & $\begin{array}{c}\text { T0 + } \\
\text { t_A2 + } \\
\text { t_AB1 + } \\
\text { T203 }\end{array}$ & $\begin{array}{c}\text { UE B timer } \\
\text { T203 } \\
\text { expires }\end{array}$ & - & O: silence & - & $\begin{array}{c}\text { UE B stops } \\
\text { rendering RTP } \\
\text { packets, clears } \\
\text { stored SSRC of } \\
\text { current } \\
\text { arbitrator, starts } \\
\text { T230 }\end{array}$ & 7.2.3.4.4 \\
\hline
\end{tabular}

Note: Due to the independence (not synchronized) of the timers T203 in the UEs, the ordering of step 6 and step 7 may be reversed. We intentionally terminate the scenario at this point and do not follow through with the reception of UE C's Floor Request message by UE A or UE B.

\subsubsection{Floor release by pre-empted floor arbitrator}

In this scenario, two users request the floor. Queuing is not supported. The floor is preemptively granted to one of them, who shortly afterwards releases it (before sending any RTP media).

Scenario Purpose: To observe that a user can pre-emptively request the floor and have it granted to him, that a new user tries to request it as well, and then finally that the floor arbitrator eventually releases the floor (before sending RTP media).

Table 11: Floor release by pre-empted floor arbitrator

\begin{tabular}{|c|c|c|c|c|c|c|c|}
\hline & Timeline & $\begin{array}{c}\text { Triggering } \\
\text { Event }\end{array}$ & $\begin{array}{c}\text { UE A state } \\
\text { transition }\end{array}$ & $\begin{array}{c}\text { UE B State } \\
\text { transition }\end{array}$ & $\begin{array}{c}\text { UE C State } \\
\text { transition }\end{array}$ & $\begin{array}{c}\text { Consequent } \\
\text { behavior }\end{array}$ & $\begin{array}{c}\text { Reference } \\
\text { for details }\end{array}$ \\
\hline 0 & - & - & $\begin{array}{c}\text { O: has } \\
\text { permission }\end{array}$ & $\begin{array}{c}\text { O: has no } \\
\text { permission }\end{array}$ & $\begin{array}{c}\text { O: has no } \\
\text { permission }\end{array}$ & - & - \\
\hline 1 & T0 & $\begin{array}{c}\text { UE B } \\
\text { pushes PTT } \\
\text { button }\end{array}$ & - & $\begin{array}{c}\text { O: } \\
\text { pending } \\
\text { request }\end{array}$ & - & $\begin{array}{c}\text { UE B sends } \\
\text { Floor Request, } \\
\text { starts T201 and } \\
\text { initializes C201 }\end{array}$ & $\mathbf{7 . 2 . 3 . 4 . 2}$ \\
\hline
\end{tabular}




\begin{tabular}{|c|c|c|c|c|c|c|c|}
\hline & Timeline & $\begin{array}{c}\text { Triggering } \\
\text { Event }\end{array}$ & $\begin{array}{l}\text { UE A state } \\
\text { transition }\end{array}$ & $\begin{array}{l}\text { UE B State } \\
\text { transition }\end{array}$ & $\begin{array}{l}\text { UE C State } \\
\text { transition }\end{array}$ & $\begin{array}{c}\text { Consequent } \\
\text { behavior }\end{array}$ & $\begin{array}{l}\text { Reference } \\
\text { for details }\end{array}$ \\
\hline 2 & $\begin{array}{l}\text { T0 + } \\
\text { t_BA1 }\end{array}$ & $\begin{array}{l}\text { UE A and } \\
\text { UE C } \\
\text { receive } \\
\text { Floor } \\
\text { Request }\end{array}$ & $\begin{array}{c}\text { O: } \\
\text { pending } \\
\text { granted }\end{array}$ & - & - & $\begin{array}{c}\text { UE A stops } \\
\text { sending RTP } \\
\text { packets, sends } \\
\text { Floor Granted, } \\
\text { stops T206 and } \\
\text { T207, starts } \\
\text { T205 and } \\
\text { initializes C205; } \\
\text { UE C discards } \\
\text { the floor control } \\
\text { message }\end{array}$ & $\begin{array}{c}\text { 7.2.3.5.7 } \\
\text { 7.2.3.1 }\end{array}$ \\
\hline 3 & $\begin{array}{c}\text { T0 + } \\
\mathrm{t} \_\mathrm{BA} 1+ \\
\mathrm{t} \_\mathrm{AB} 1\end{array}$ & $\begin{array}{l}\text { UE B and } \\
\text { UE C } \\
\text { receive } \\
\text { Floor } \\
\text { Granted }\end{array}$ & - & $\begin{array}{c}\text { O: has } \\
\text { permission }\end{array}$ & - & $\begin{array}{l}\text { UE B stops } \\
\text { rendering RTP } \\
\text { packets, and } \\
\text { stops T201 and } \\
\text { T203; } \\
\text { UE C stops } \\
\text { rendering RTP } \\
\text { packets, sets } \\
\text { stored SSRC of } \\
\text { candidate floor } \\
\text { arbitrator to } \\
\text { SSRC of UE B, } \\
\text { and starts T203 }\end{array}$ & $\begin{array}{l}\text { 7.2.3.6.7 } \\
\text { 7.2.3.4.5 }\end{array}$ \\
\hline 4 & $\begin{array}{c}\text { T0+ } \\
\text { t_C1 } \\
(\text { with } \\
t \_C 1> \\
t \_B A 1+ \\
\left.t \_A B 1\right)\end{array}$ & $\begin{array}{c}\text { UE C } \\
\text { pushes PTT } \\
\text { button }\end{array}$ & - & - & $\begin{array}{l}\text { O: pending } \\
\text { request }\end{array}$ & $\begin{array}{l}\text { UE C sends } \\
\text { Floor Request, } \\
\text { starts T201 and } \\
\text { initializes C201 }\end{array}$ & 7.2.3.4.2 \\
\hline 5 & $\begin{array}{l}\mathrm{T} 0+ \\
\mathrm{t} \_\mathrm{C} 1+ \\
\mathrm{t} \_\mathrm{CA} 1\end{array}$ & $\begin{array}{l}\text { UE A and } \\
\text { UE B } \\
\text { receive } \\
\text { Floor } \\
\text { Request } \\
\end{array}$ & - & - & - & $\begin{array}{l}\text { UE A sends } \\
\text { Floor Deny; } \\
\text { UE B sends } \\
\text { Floor Deny }\end{array}$ & $\begin{array}{c}\text { 7.2.3.7.10 } \\
\text { 7.2.3.5.4 }\end{array}$ \\
\hline 6 & $\begin{array}{c}\text { T0 + } \\
\text { t_C1 + } \\
\text { t_CA1 + } \\
\text { t_AB2 }\end{array}$ & $\begin{array}{l}\text { UE B and } \\
\text { UE C } \\
\text { receive } \\
\text { Floor Deny } \\
\text { (from } \boldsymbol{U E} \\
\boldsymbol{A}) \\
\end{array}$ & - & - & $\begin{array}{c}\text { O: has no } \\
\text { permission }\end{array}$ & $\begin{array}{l}\text { UE B discards } \\
\text { the floor control } \\
\text { message; } \\
\text { UE C stops } \\
\text { T201, and starts } \\
\text { T203 }\end{array}$ & $\begin{array}{c}\text { 7.2.3.1 } \\
\text { 7.2.3.6.4 }\end{array}$ \\
\hline 7 & $\begin{array}{c}\text { T0 + } \\
\text { t_C1 + } \\
\text { t_CA1 + } \\
\text { t_BA2 } \\
(\text { with } \\
t \_B A 2> \\
\left.t \_A B 2\right)\end{array}$ & $\begin{array}{l}\mathbf{U E} \mathbf{A} \text { and } \\
\text { UE C } \\
\text { receive } \\
\text { Floor Deny } \\
\text { (from } \boldsymbol{U E} \\
\boldsymbol{B} \text { ) }\end{array}$ & - & - & - & $\begin{array}{l}\text { UE A and UE C } \\
\text { discard the floor } \\
\text { control message }\end{array}$ & 7.2.3.1 \\
\hline
\end{tabular}




\begin{tabular}{|c|c|c|c|c|c|c|c|}
\hline & Timeline & $\begin{array}{c}\text { Triggering } \\
\text { Event }\end{array}$ & $\begin{array}{l}\text { UE A state } \\
\text { transition }\end{array}$ & $\begin{array}{c}\text { UE B State } \\
\text { transition }\end{array}$ & $\begin{array}{l}\text { UE C State } \\
\text { transition }\end{array}$ & $\begin{array}{c}\text { Consequent } \\
\text { behavior }\end{array}$ & $\begin{array}{l}\text { Reference } \\
\text { for details }\end{array}$ \\
\hline 8 & $\begin{array}{c}\text { T0 + } \\
\text { t_B1 } \\
(\text { with } \\
t \_B 1> \\
\text { max } \\
\left(t \_C 1+\right. \\
t \_C A 1+ \\
t \_A B 2 ; \\
t \_C 1+ \\
t \_C A 1+ \\
\left.\left.t \_B A 2\right)\right) \\
\end{array}$ & $\begin{array}{c}\text { UE B } \\
\text { releases } \\
\text { PTT button }\end{array}$ & - & O: silence & - & $\begin{array}{c}\text { UE B clears } \\
\text { stored SSRC of } \\
\text { current } \\
\text { arbitrator, sends } \\
\text { Floor Release, } \\
\text { stops T206 and } \\
\text { T207, and starts } \\
\text { T230 }\end{array}$ & 7.2.3.5.5 \\
\hline 9 & $\begin{array}{l}\text { T0 + } \\
\text { t_B1 + } \\
\text { t_BA3 }\end{array}$ & $\begin{array}{l}\text { UE A and } \\
\text { UE C } \\
\text { receive } \\
\text { Floor } \\
\text { Release }\end{array}$ & - & - & O: silence & $\begin{array}{l}\text { Since queuing is } \\
\text { not supported, } \\
\text { UE A remains } \\
\text { in its current } \\
\text { state; } \\
\text { UE C clears } \\
\text { SSRC of current } \\
\text { floor arbitrator } \\
\text { and candidate } \\
\text { arbitrator, stops } \\
\text { T203, and starts } \\
\text { T230 } \\
\end{array}$ & $\begin{array}{l}\text { 7.2.3.7.9 } \\
\text { 7.2.3.4.3 }\end{array}$ \\
\hline 10 & $\begin{array}{c}\text { T0 + } \\
\text { t_BA1 + } \\
\text { T205 }\end{array}$ & $\begin{array}{c}\text { UE A T205 } \\
\text { expires }\end{array}$ & - & - & - & $\begin{array}{l}\text { UE A sends } \\
\text { Floor Granted, } \\
\text { starts T205 and } \\
\text { increments } \\
\text { C205 }\end{array}$ & 7.2.3.7.3 \\
\hline 11 & $\begin{array}{c}\text { T0 + } \\
\text { t_BA1 + } \\
\text { T205 + } \\
\text { t_AB3 }\end{array}$ & $\begin{array}{l}\text { UE B and } \\
\text { UE C } \\
\text { receive } \\
\text { Floor } \\
\text { Granted }\end{array}$ & - & - & $\begin{array}{c}\text { O: has no } \\
\text { permission }\end{array}$ & $\begin{array}{l}\text { UE B discards } \\
\text { the floor control } \\
\text { message; } \\
\text { UE C sets } \\
\text { SSRC of } \\
\text { candidate floor } \\
\text { arbitrator to } \\
\text { SSRC of UE B, } \\
\text { stops T230, and } \\
\text { starts T203 }\end{array}$ & $\begin{array}{c}\text { 7.2.3.1 } \\
7.2 .3 .3 .4\end{array}$ \\
\hline 12 & $\begin{array}{c}\mathrm{T} 0+ \\
\mathrm{t} \_\mathrm{BA} 1+ \\
\mathrm{n} * \mathrm{~T} 205\end{array}$ & $\begin{array}{l}\text { UE A T205 } \\
\text { expires } \\
\text { (C205 at its } \\
\text { upper limit) }\end{array}$ & O: silence & - & - & $\begin{array}{l}\text { UE A clears } \\
\text { SSRC of current } \\
\text { floor arbitrator, } \\
\text { starts T230, and } \\
\text { resets C205 }\end{array}$ & 7.2.3.7.5 \\
\hline 13 & $\begin{array}{c}\text { T0 + } \\
\text { t_BA1 + } \\
\text { T205 + } \\
\text { t_AB3 + } \\
\text { T203 }\end{array}$ & $\begin{array}{c}\text { UE C T203 } \\
\text { expires }\end{array}$ & - & - & O: silence & $\begin{array}{l}\text { UE C stops } \\
\text { rendering RTP } \\
\text { packets, clears } \\
\text { SSRC of current } \\
\text { floor arbitrator, } \\
\text { and starts T230 }\end{array}$ & 7.2.3.4.4 \\
\hline
\end{tabular}

And the corresponding figure is Fig. 7: 


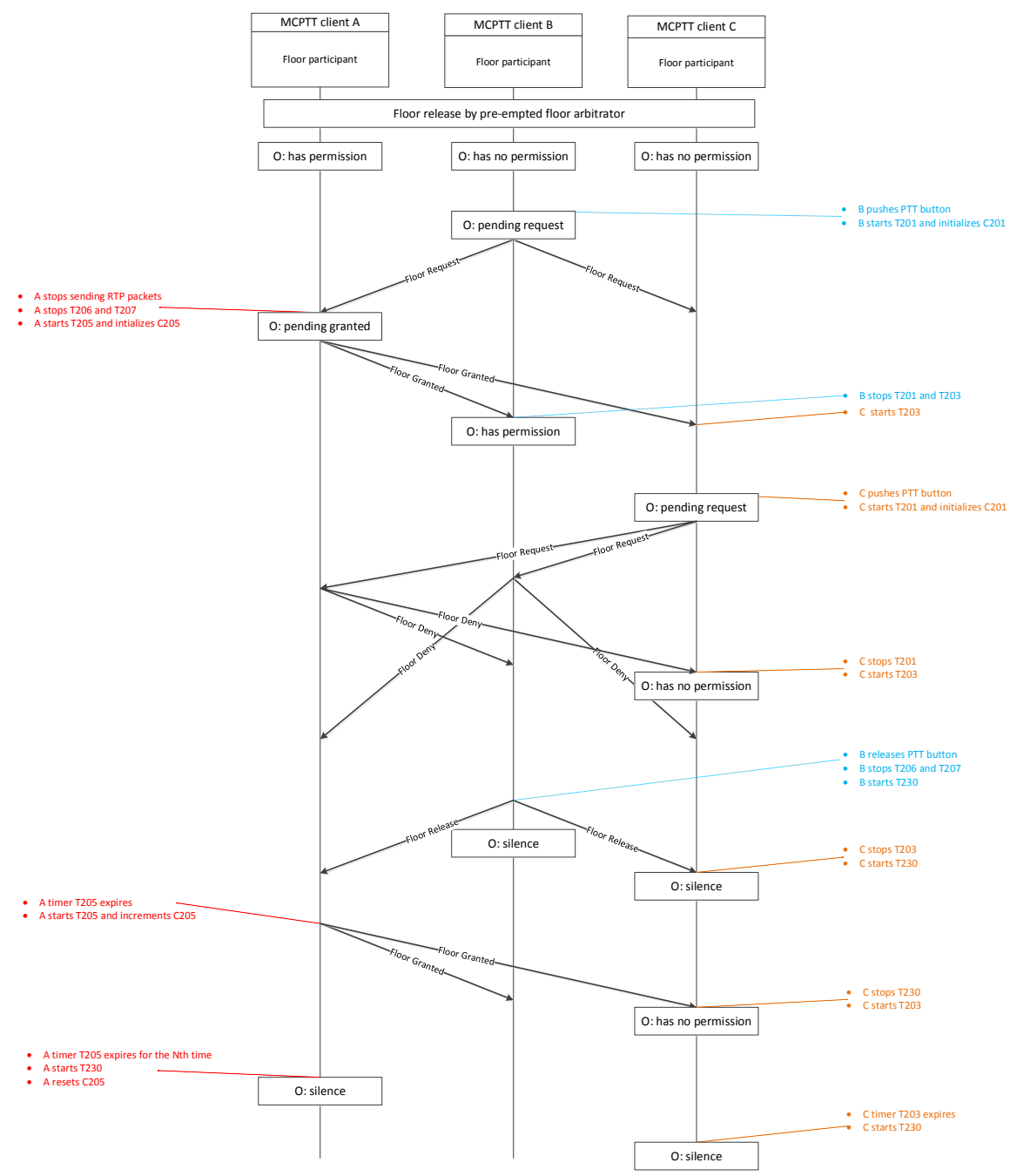

Figure 7: Floor release by pre-empted floor arbitrator

\subsubsection{Session initialization}

\subsubsection{Session initialization - normal}

This scenario focuses on the initialization of the MCPTT session. It covers the creation of the floor control entity and the sending of the first data packet after the call starts.

Scenario Purpose: To observe that the originating MCPTT call participant becomes arbitrator (Floor Grant) and starts transmitting (RTP packets). 
Table 12: Session initialization - normal

\begin{tabular}{|c|c|c|c|c|c|c|c|}
\hline & Timeline & $\begin{array}{c}\text { Triggering } \\
\text { event }\end{array}$ & $\begin{array}{l}\text { UE A state } \\
\text { transition }\end{array}$ & $\begin{array}{l}\text { UE B state } \\
\text { transition }\end{array}$ & $\begin{array}{l}\text { UE C state } \\
\text { transition }\end{array}$ & $\begin{array}{c}\text { Consequent } \\
\text { behavior }\end{array}$ & $\begin{array}{c}\text { Reference } \\
\text { for } \\
\text { details } \\
\end{array}$ \\
\hline 0 & & & Start-stop & Start-stop & Start-stop & - & - \\
\hline 1 & T0 & $\begin{array}{l}\text { UE A, UE } \\
\text { B and UE } \\
\text { C receive } \\
\text { from call } \\
\text { control } \\
\text { entity a } \\
\text { request to } \\
\text { initiate } \\
\text { Floor } \\
\text { Control }\end{array}$ & $\begin{array}{c}\text { O: has } \\
\text { permission }\end{array}$ & O: silence & O: silence & $\begin{array}{l}\text { UE A creates an } \\
\text { instance of Floor } \\
\text { Control } \\
\text { Participant, and } \\
\text { sends Floor } \\
\text { Granted; } \\
\text { UE B and UE C } \\
\text { create an } \\
\text { instance of Floor } \\
\text { Control } \\
\text { Participant, and } \\
\text { start T230 } \\
\end{array}$ & $\begin{array}{l}7.2 .3 .2 .2 \\
7.2 .3 .2 .3\end{array}$ \\
\hline 2 & $\begin{array}{c}\mathrm{T} 0+ \\
\mathrm{t} \_\mathrm{AB} 1\end{array}$ & $\begin{array}{l}\text { UE B and } \\
\text { UE C } \\
\text { receives } \\
\text { Floor } \\
\text { Granted }\end{array}$ & - & $\begin{array}{l}\text { O: has no } \\
\text { permission }\end{array}$ & $\begin{array}{l}\text { O: has no } \\
\text { permission }\end{array}$ & $\begin{array}{l}\text { UE B and UE C } \\
\text { start T203, stop } \\
\text { T230, and set } \\
\text { SSRC of current } \\
\text { arbitrator to the } \\
\text { SSRC of user } \\
\text { (UE A) whom } \\
\text { the floor was } \\
\text { granted to }\end{array}$ & 7.2.3.3.4 \\
\hline 3 & $\begin{array}{l}\text { T0+ } \\
\mathrm{t} \_\mathrm{A} 1 \\
\text { (with } \\
\text { t_A1 > } \\
\left.t \_A B 1\right) \\
\end{array}$ & $\begin{array}{c}\text { UE A } \\
\text { sends RTP } \\
\text { packets }\end{array}$ & - & - & - & $\begin{array}{c}\text { UE A starts } \\
\text { T206 }\end{array}$ & 7.2.3.5.2 \\
\hline 4 & $\begin{array}{c}\text { T0 + } \\
\text { t_A1 }+ \\
\text { t_AB2 }\end{array}$ & $\begin{array}{l}\text { UE B and } \\
\text { UE C } \\
\text { receive } \\
\text { RTP } \\
\text { packets }\end{array}$ & - & - & - & $\begin{array}{c}\text { UE B and UE C } \\
\text { restart T203 and } \\
\text { set SSRC of } \\
\text { current arbitrator } \\
\text { to SSRC of RTP } \\
\text { packet } \\
\end{array}$ & 7.2.3.4.6 \\
\hline
\end{tabular}

And the corresponding figure is Fig. 8: 


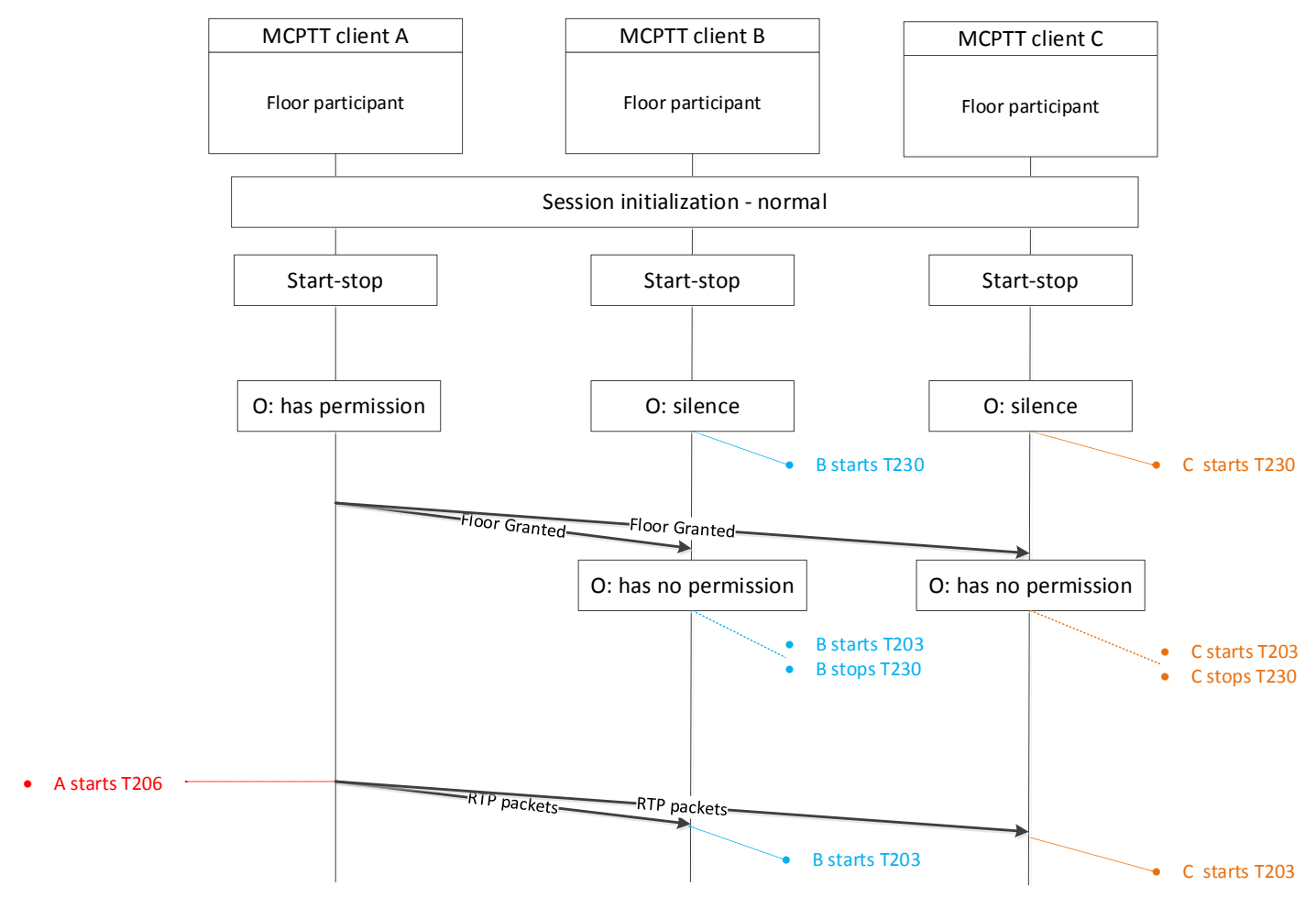

Figure 8: Session initialization - normal

\subsubsection{Session initialization - message lost}

Similar to the scenario in Section 2.1.3.1, this scenario focuses on the initialization of the MCPTT session. It covers the creation of the floor control entity and the sending of the first data packet after the call starts. However, the floor granted message sent by UE A at time T0 is lost and does not reach UE B or UE C. UE B and UE C receive the RTP packet first instead.

Scenario Purpose: To observe that a UE can start receiving and rendering RTP packets from arbitrator.

Table 13: Session initialization - message lost

\begin{tabular}{|c|c|c|c|c|c|c|c|}
\hline & Timeline & $\begin{array}{c}\text { Triggering } \\
\text { event }\end{array}$ & $\begin{array}{c}\text { UE A state } \\
\text { transition }\end{array}$ & $\begin{array}{c}\text { UE B state } \\
\text { transition }\end{array}$ & $\begin{array}{c}\text { UE C state } \\
\text { transition }\end{array}$ & $\begin{array}{c}\text { Consequent } \\
\text { behavior }\end{array}$ & $\begin{array}{c}\text { Reference } \\
\text { for details }\end{array}$ \\
\hline 0 & - & - & Start-stop & Start-stop & Start-stop & - & - \\
\hline
\end{tabular}




\begin{tabular}{|c|c|c|c|c|c|c|c|}
\hline & Timeline & $\begin{array}{c}\text { Triggering } \\
\text { event }\end{array}$ & $\begin{array}{l}\text { UE A state } \\
\text { transition }\end{array}$ & $\begin{array}{l}\text { UE B state } \\
\text { transition }\end{array}$ & $\begin{array}{l}\text { UE C state } \\
\text { transition }\end{array}$ & $\begin{array}{c}\text { Consequent } \\
\text { behavior }\end{array}$ & $\begin{array}{l}\text { Reference } \\
\text { for details }\end{array}$ \\
\hline 1 & T0 & $\begin{array}{c}\text { UE A, UE } \\
\text { B and UE C } \\
\text { receive from } \\
\text { call control } \\
\text { entity } \\
\text { request to } \\
\text { initiate } \\
\text { Floor } \\
\text { Control }\end{array}$ & $\begin{array}{c}\text { O: has } \\
\text { permission }\end{array}$ & O: silence & O: silence & $\begin{array}{c}\text { UE A creates an } \\
\text { instance of Floor } \\
\text { Control } \\
\text { Participant, and } \\
\text { sends Floor } \\
\text { Granted; } \\
\text { UE B and UE C } \\
\text { create an } \\
\text { instance of Floor } \\
\text { Control } \\
\text { Participant, and } \\
\text { start T230 } \\
\end{array}$ & $\begin{array}{l}\text { 7.2.3.2.2 } \\
\text { 7.2.3.2.3 }\end{array}$ \\
\hline 2 & $\begin{array}{l}\text { T0 + } \\
\text { t_A1 }\end{array}$ & $\begin{array}{l}\text { UE A sends } \\
\text { RTP packet }\end{array}$ & - & - & - & $\begin{array}{c}\text { UE A starts } \\
\text { T206 }\end{array}$ & 7.2.3.5.2 \\
\hline 3 & $\begin{array}{c}\mathrm{T} 0+ \\
\text { t_A1 + } \\
\text { t_AB1 }\end{array}$ & $\begin{array}{l}\text { UE B and } \\
\text { UE C } \\
\text { receive RTP } \\
\text { packet }\end{array}$ & - & $\begin{array}{l}\text { O: has no } \\
\text { permission }\end{array}$ & $\begin{array}{l}\text { O: has no } \\
\text { permission }\end{array}$ & $\begin{array}{c}\text { UE B and UE C } \\
\text { stop T230, start } \\
\text { T203, and set } \\
\text { SSRC of current } \\
\text { arbitrator to } \\
\text { SSRC (UE A) of } \\
\text { RTP packet } \\
\end{array}$ & 7.2.3.3.3 \\
\hline
\end{tabular}

And the corresponding figure is Fig. 9:

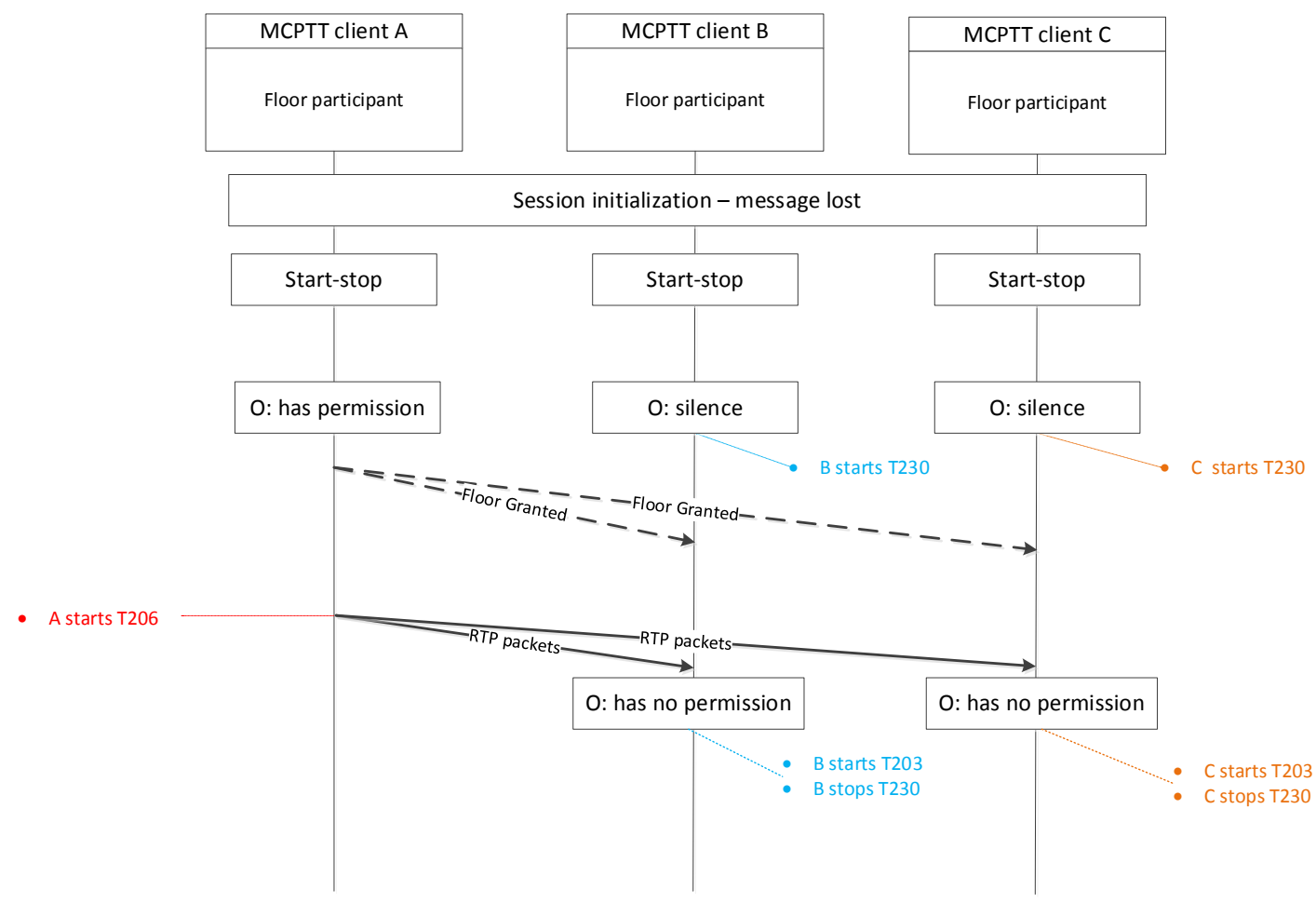

Figure 9: Session initialization - message lost 


\subsubsection{Session initialization - private call}

This scenario focuses on the initialization of the MCPTT session for a private call. It covers the creation of the floor control entity and the sending of the first data packet after the call starts, as well as the floor arbitrator giving back the floor to the second user once it is done talking.

Scenario purpose: To observe that the originating MCPTT call participant becomes arbitrator (Floor Grant) of the private call and starts transmitting (RTP packets), then once done talking, hands over the floor to the other user.

Table 14: Session initialization - private call

\begin{tabular}{|c|c|c|c|c|c|c|}
\hline & Timeline & $\begin{array}{c}\text { Triggering } \\
\text { event }\end{array}$ & $\begin{array}{l}\text { UE A state } \\
\text { transition }\end{array}$ & $\begin{array}{l}\text { UE B state } \\
\text { transition }\end{array}$ & Consequent behavior & $\begin{array}{l}\text { Reference } \\
\text { for details }\end{array}$ \\
\hline 0 & & & Start-stop & Start-stop & - & - \\
\hline 1 & T0 & $\begin{array}{c}\text { UE } \mathbf{A} \text { and } \mathbf{U E} \\
\mathbf{B} \text { receive from } \\
\text { call control } \\
\text { entity a request } \\
\text { to initiate } \\
\text { Floor Control }\end{array}$ & $\begin{array}{c}\text { O: has } \\
\text { permission }\end{array}$ & $\begin{array}{c}\text { O: has no } \\
\text { permission }\end{array}$ & $\begin{array}{c}\text { UE A creates an } \\
\text { instance of Floor } \\
\text { Control Participant, } \\
\text { and sends Floor } \\
\text { Granted; } \\
\text { UE B creates an } \\
\text { instance of Floor } \\
\text { Control Participant, } \\
\text { and starts T203 } \\
\end{array}$ & $\begin{array}{l}7.2 .3 .2 .2 \\
7.2 .3 .2 .4\end{array}$ \\
\hline 2 & $\mathrm{~T} 0+\mathrm{t} \_\mathrm{AB} 1$ & $\begin{array}{l}\text { UE B receives } \\
\text { Floor Granted }\end{array}$ & - & - & $\begin{array}{l}\text { UE B stops rendering } \\
\text { RTP packets, sets } \\
\text { SSRC of candidate } \\
\text { arbitrator to SSRC of } \\
\text { UE A, and restarts } \\
\text { T203 } \\
\end{array}$ & 7.2.3.4.5 \\
\hline 3 & $\begin{array}{l}\mathrm{T} 0+\mathrm{t} \_\mathrm{A} 1 \\
\left(\text { with } t \_A 1\right. \\
\left.>+t \_A B 1\right)\end{array}$ & $\begin{array}{l}\text { UE A sends } \\
\text { RTP media } \\
\text { packets }\end{array}$ & - & - & UE A starts T206 & 7.2.3.5.2 \\
\hline 4 & $\begin{array}{l}\text { T0 + t_A1 } \\
+\mathrm{t} \_\mathrm{AB} 2\end{array}$ & $\begin{array}{l}\text { UE B receives } \\
\text { RTP packets }\end{array}$ & - & - & $\begin{array}{l}\text { UE B renders receives } \\
\text { RTP packets, sets } \\
\text { stored SSRC of } \\
\text { current arbitrator to } \\
\text { SSRC of UE A, clears } \\
\text { stores SSRC of } \\
\text { candidate arbitrator, } \\
\text { and restarts T203 } \\
\end{array}$ & 7.2.3.4.6 \\
\hline 5 & $\begin{array}{c}\mathrm{T} 0+\mathrm{t} \_\mathrm{A} 2 \\
\left(\text { with } t \_A 2\right. \\
>+t \_A 1+ \\
\left.t \_A B 2\right)\end{array}$ & $\begin{array}{l}\text { UE A releases } \\
\text { PTT button }\end{array}$ & O: silence & - & $\begin{array}{c}\text { UE A sends Floor } \\
\text { Release, stops T206 } \\
\text { and T207, clears } \\
\text { SSRC of current floor } \\
\text { arbitrator, and starts } \\
\text { T230 }\end{array}$ & 7.2.3.5.5 \\
\hline
\end{tabular}




\begin{tabular}{|c|c|c|c|c|c|c|}
\hline & Timeline & $\begin{array}{c}\text { Triggering } \\
\text { event }\end{array}$ & $\begin{array}{l}\text { UE A state } \\
\text { transition }\end{array}$ & $\begin{array}{l}\text { UE B state } \\
\text { transition }\end{array}$ & Consequent behavior & $\begin{array}{l}\text { Reference } \\
\text { for details }\end{array}$ \\
\hline 6 & $\begin{array}{c}\text { T0 + t_A2 } \\
+ \text { t_AB3 }\end{array}$ & $\begin{array}{l}\text { UE B receives } \\
\text { Floor Release }\end{array}$ & - & O: silence & $\begin{array}{l}\text { UE B stops rendering } \\
\text { RTP packets, clears } \\
\text { SSRC of current floor } \\
\text { arbitrator, stops T203 } \\
\text { and starts T230 }\end{array}$ & 7.2.3.4.3 \\
\hline 7 & $\begin{array}{c}\mathrm{T} 0+\mathrm{t} \_\mathrm{B} 1 \\
\left(\text { with } t \_B 1\right. \\
>+t \_A 2+ \\
\left.t \_A B 3\right)\end{array}$ & $\begin{array}{c}\text { UE B presses } \\
\text { PTT button }\end{array}$ & - & $\begin{array}{l}\text { O: pending } \\
\text { request }\end{array}$ & $\begin{array}{l}\text { UE B sends Floor } \\
\text { Request, starts T201 } \\
\text { and initializes C201, } \\
\text { and stops T230 }\end{array}$ & 7.2.3.3.2 \\
\hline 8 & $\begin{array}{c}\text { T0 + t_B1 } \\
+ \text { t_BA1 }\end{array}$ & $\begin{array}{l}\text { UE A receives } \\
\text { Floor Request }\end{array}$ & $\begin{array}{l}\text { O: pending } \\
\text { granted }\end{array}$ & - & $\begin{array}{l}\text { UE A sends Floor } \\
\text { Granted, stops T230 } \\
\text { and starts T205 }\end{array}$ & 7.2.3.3.5 \\
\hline 9 & $\begin{array}{l}\text { T0 + t_B1 } \\
+\mathrm{t} \_\mathrm{BA} 1+ \\
\text { t_AB4 }\end{array}$ & $\begin{array}{l}\text { UE B receives } \\
\text { Floor Granted }\end{array}$ & - & $\begin{array}{c}\text { O: has } \\
\text { permission }\end{array}$ & $\begin{array}{l}\text { UE B stops rendering } \\
\text { RTP packets, sets } \\
\text { SSRC of current floor } \\
\text { arbitrator to own } \\
\text { SSRC, and stops T201 } \\
\text { and T203 } \\
\end{array}$ & 7.2.3.6.7 \\
\hline
\end{tabular}

And the corresponding figure is Fig. 10: 


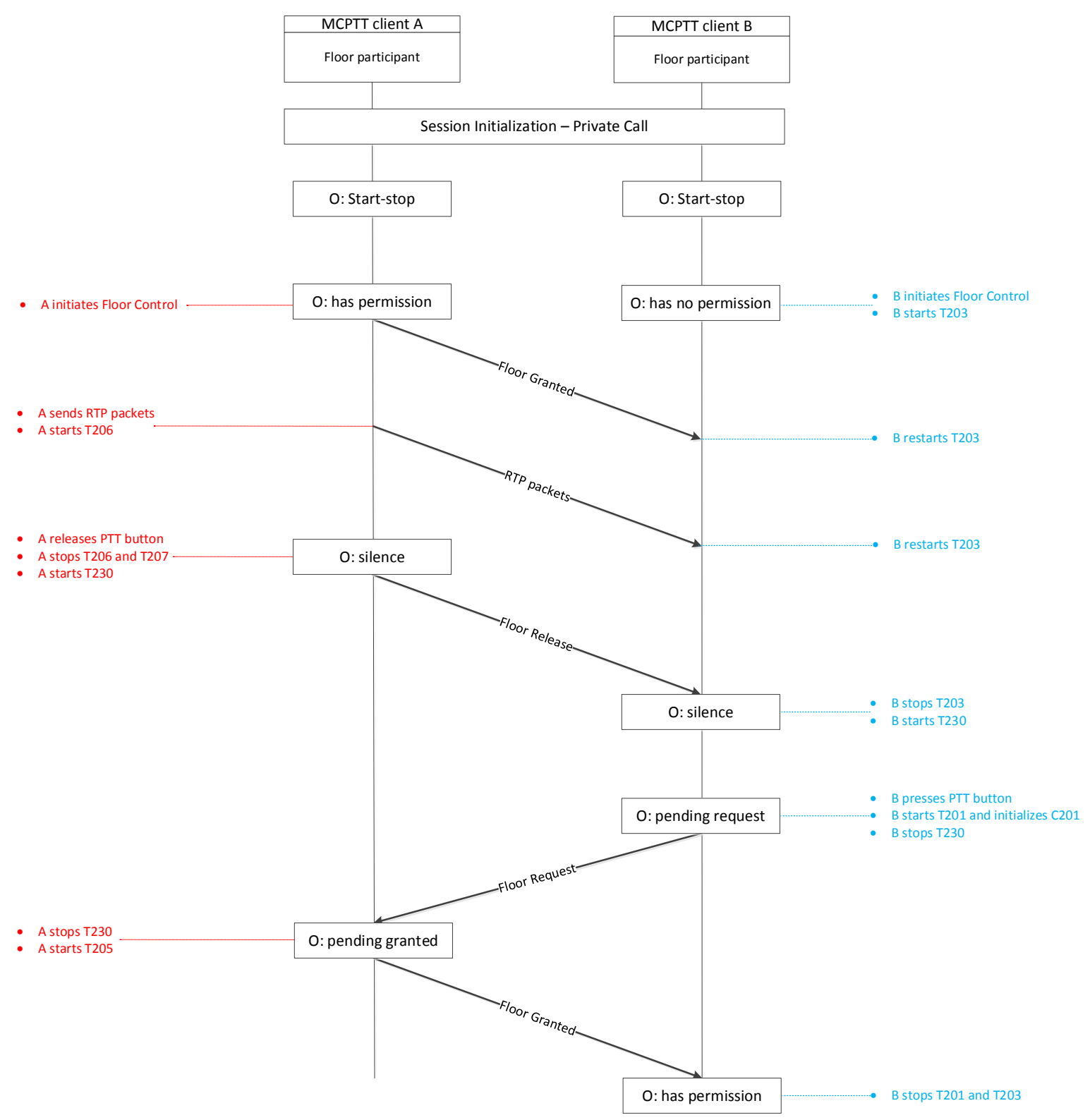

Figure 10: Session initialization - private call

\subsubsection{Session initialization - broadcast call}

This scenario focuses on the initialization of the MCPTT session for a broadcast call.

Scenario purpose: To observe that the originating MCPTT call participant becomes arbitrator (Floor Grant) of the broadcast call. 
Table 15: Session initialization - broadcast call

\begin{tabular}{|c|c|c|c|c|c|c|c|}
\hline & Timeline & $\begin{array}{c}\text { Triggering } \\
\text { event }\end{array}$ & $\begin{array}{l}\text { UE A state } \\
\text { transition }\end{array}$ & $\begin{array}{l}\text { UE B state } \\
\text { transition }\end{array}$ & $\begin{array}{l}\text { UE C state } \\
\text { transition }\end{array}$ & $\begin{array}{c}\text { Consequent } \\
\text { behavior }\end{array}$ & $\begin{array}{c}\text { Reference } \\
\text { for } \\
\text { details }\end{array}$ \\
\hline 0 & & & Start-stop & Start-stop & Start-stop & - & - \\
\hline 1 & T0 & $\begin{array}{l}\text { UE A, UE } \\
\text { B and UE } \\
\text { C receive } \\
\text { from call } \\
\text { control } \\
\text { entity a } \\
\text { request to } \\
\text { initiate } \\
\text { Floor } \\
\text { Control }\end{array}$ & $\begin{array}{c}\text { O: has } \\
\text { permission }\end{array}$ & $\begin{array}{c}\text { O: has no } \\
\text { permission }\end{array}$ & $\begin{array}{l}\text { O: has no } \\
\text { permission }\end{array}$ & $\begin{array}{c}\text { UE A } \\
\text { creates an } \\
\text { instance of } \\
\text { Floor } \\
\text { Control } \\
\text { Participant, } \\
\text { and sends } \\
\text { Floor } \\
\text { Granted; } \\
\text { UE B and } \\
\text { UE C create } \\
\text { an instance } \\
\text { of Floor } \\
\text { Control } \\
\text { Participant, } \\
\text { and start } \\
\text { T203 }\end{array}$ & $\begin{array}{l}\text { 7.2.3.2.2 } \\
\text { 7.2.3.2.9 }\end{array}$ \\
\hline 2 & $\mathrm{~T} 0+\mathrm{t} \_\mathrm{AB} 1$ & $\begin{array}{l}\text { UE B and } \\
\text { UE C } \\
\text { receive } \\
\text { Floor } \\
\text { Granted }\end{array}$ & - & - & & $\begin{array}{c}\text { UE B and } \\
\text { UE C set } \\
\text { SSRC of } \\
\text { candidate } \\
\text { arbitrator to } \\
\text { SSRC of } \\
\text { UE A, and } \\
\text { restart T203 }\end{array}$ & 7.2.3.4.5 \\
\hline
\end{tabular}

\subsubsection{Session release}

This scenario focuses on the release of the MCPTT session, including the deletion of the floor control entity.

Scenario Purpose: To observe that the floor control state machine is terminated when the call is terminated.

Table 16: Session release

\begin{tabular}{|c|c|c|c|c|c|c|c|}
\hline Timeline & $\begin{array}{c}\text { Triggering } \\
\text { event }\end{array}$ & $\begin{array}{c}\text { UE A } \\
\text { state } \\
\text { transition }\end{array}$ & $\begin{array}{c}\text { UE B state } \\
\text { transition }\end{array}$ & $\begin{array}{c}\text { UE C state } \\
\text { transition }\end{array}$ & $\begin{array}{c}\text { Consequent } \\
\text { behavior }\end{array}$ & $\begin{array}{c}\text { Reference } \\
\text { for } \\
\text { details }\end{array}$ \\
\hline 0 & - & - & Any state & Any state & Any state & - & - \\
\hline
\end{tabular}




\begin{tabular}{|c|c|c|c|c|c|c|c|}
\hline & Timeline & $\begin{array}{c}\text { Triggering } \\
\text { event }\end{array}$ & $\begin{array}{c}\text { UE A } \\
\text { state } \\
\text { transition }\end{array}$ & $\begin{array}{l}\text { UE B state } \\
\text { transition }\end{array}$ & $\begin{array}{l}\text { UE C state } \\
\text { transition }\end{array}$ & $\begin{array}{c}\text { Consequent } \\
\text { behavior }\end{array}$ & $\begin{array}{c}\text { Reference } \\
\text { for } \\
\text { details }\end{array}$ \\
\hline 1 & T0 & $\begin{array}{c}\text { UE A, UE } \\
\text { B, and UE } \\
\text { C receive } \\
\text { an MCPTT } \\
\text { call release } \\
\text { request }\end{array}$ & Start-stop & Start-stop & Start-stop & $\begin{array}{l}\text { UE A, UE B, and } \\
\text { UE C stop sending } \\
\text { Floor Control } \\
\text { messages towards } \\
\text { other Floor } \\
\text { participants, } \\
\text { request the } \\
\text { MCPTT client to } \\
\text { stop sending and } \\
\text { receiving RTP } \\
\text { media packets, } \\
\text { release every } \\
\text { resource and stop } \\
\text { every running } \\
\text { timer, and } \\
\text { terminate the } \\
\text { instance of Floor } \\
\text { Participant state } \\
\text { diagram }\end{array}$ & 7.2.3.9.2 \\
\hline
\end{tabular}

\subsection{Basic group call - call control}

The call control protocol is the protocol used to control the session needed to support MCPTT. It addresses issues such as how to initiate a call, how to terminate a call, and how to respond when receiving a call. An MCPTT off-network mode call may be a basic group call, a private call, or broadcast group call. Test scenarios presented in Section 2.2 and Section 2.3 are designed for basic group calls.

The state transitions that are analyzed in each test scenario of Section 2.2 are based on Figure 10.2.2.2-1 of [4]. Call Control test scenarios can be categorized into 4 groups: call setup, call merge, call release, and call reject.

\subsubsection{Call setup}

\subsubsection{Call setup - join a call}

In this scenario, a user decides to join an ongoing basic group call. It receives an answer to its group call probe and joins the call as a terminating participant.

Scenario purpose: To observe that a user joins an ongoing group call.

Table 17: Call setup - join a call

\begin{tabular}{|c|c|c|c|c|c|c|c|}
\hline & Timeline & $\begin{array}{c}\text { Triggering } \\
\text { event }\end{array}$ & $\begin{array}{c}\text { UE A state } \\
\text { transition }\end{array}$ & $\begin{array}{c}\text { UE B state } \\
\text { transition }\end{array}$ & $\begin{array}{c}\text { UE C state } \\
\text { transition }\end{array}$ & $\begin{array}{c}\text { Consequent } \\
\text { behavior }\end{array}$ & $\begin{array}{c}\text { Reference } \\
\text { for details }\end{array}$ \\
\hline 0 & - & - & $\begin{array}{c}\text { S1: start- } \\
\text { stop }\end{array}$ & $\begin{array}{c}\text { S3: part of } \\
\text { ongoing } \\
\text { call }\end{array}$ & $\begin{array}{c}\text { S3: part of } \\
\text { ongoing } \\
\text { call }\end{array}$ & - & - \\
\hline
\end{tabular}




\begin{tabular}{|c|c|c|c|c|c|c|c|}
\hline & Timeline & $\begin{array}{c}\text { Triggering } \\
\text { event }\end{array}$ & $\begin{array}{l}\text { UE A state } \\
\text { transition }\end{array}$ & $\begin{array}{l}\text { UE B state } \\
\text { transition }\end{array}$ & $\begin{array}{l}\text { UE C state } \\
\text { transition }\end{array}$ & $\begin{array}{c}\text { Consequent } \\
\text { behavior }\end{array}$ & $\begin{array}{l}\text { Reference } \\
\text { for details }\end{array}$ \\
\hline 1 & T0 & $\begin{array}{c}\text { Indication } \\
\text { from UE A } \\
\text { to join a } \\
\text { group call }\end{array}$ & $\begin{array}{c}\text { S2: } \\
\text { waiting for } \\
\text { call } \\
\text { announce } \\
\text { ment }\end{array}$ & 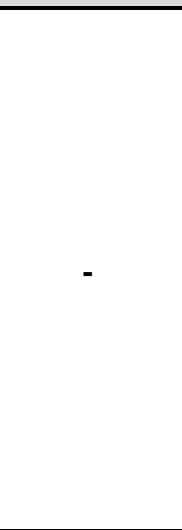 & 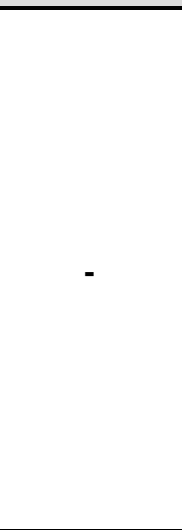 & $\begin{array}{c}\text { UE A stores } \\
\text { MCPTT group ID } \\
\text { as MCPTT group } \\
\text { ID of the call, } \\
\text { creates a Call } \\
\text { Type Control state } \\
\text { machine, } \\
\text { generates and } \\
\text { sends a GROUP } \\
\text { CALL PROBE } \\
\text { message, and } \\
\text { starts TFG1 and } \\
\text { TFG3 }\end{array}$ & 10.2 .2 .4 .2 .1 \\
\hline 2 & $\begin{array}{l}\mathrm{T} 0+ \\
\mathrm{t} \_\mathrm{AB} 1\end{array}$ & $\begin{array}{l}\text { UE B and } \\
\text { UE C } \\
\text { receive } \\
\text { GROUP } \\
\text { CALL } \\
\text { PROBE }\end{array}$ & - & - & - & $\begin{array}{l}\text { UE B and UE C } \\
\text { check if MCPTT } \\
\text { group ID IE } \\
\text { matches stored } \\
\text { MCPTT Group ID } \\
\text { of the call, stop } \\
\text { TFG2, start } \\
\text { TFG2, and set } \\
\text { stored probe } \\
\text { response of the } \\
\text { call to "true" }\end{array}$ & 10.2 .2 .4 .2 .3 \\
\hline 3 & $\begin{array}{c}\text { T0 + } \\
\text { t_AB1 + } \\
\text { TFG2 } \\
\text { (with } \\
\text { TFG2 in } \\
\text { UE B < } \\
\text { TFG2 in } \\
\text { UE C) }\end{array}$ & $\begin{array}{c}\text { UE B } \\
\text { TFG2 } \\
\text { expires }\end{array}$ & - & - & - & $\begin{array}{c}\text { UE B generates } \\
\text { and sends } \\
\text { GROUP CALL } \\
\text { ANNOUNCEME } \\
\text { NT, sets stored } \\
\text { probe response } \\
\text { value of the call to } \\
\text { "false", and starts } \\
\text { TFG2 }\end{array}$ & 10.2 .2 .4 .4 .1 \\
\hline
\end{tabular}




\begin{tabular}{|c|c|c|c|c|c|c|c|}
\hline & Timeline & $\begin{array}{c}\text { Triggering } \\
\text { event }\end{array}$ & $\begin{array}{l}\text { UE A state } \\
\text { transition }\end{array}$ & $\begin{array}{l}\text { UE B state } \\
\text { transition }\end{array}$ & $\begin{array}{l}\text { UE C state } \\
\text { transition }\end{array}$ & $\begin{array}{c}\begin{array}{c}\text { Consequent } \\
\text { behavior }\end{array} \\
\end{array}$ & $\begin{array}{l}\text { Reference } \\
\text { for details }\end{array}$ \\
\hline 4 & $\begin{array}{c}\text { T0 + } \\
\text { t_AB1 + } \\
\text { TFG2 + } \\
\text { t_BA1 }\end{array}$ & $\begin{array}{l}\text { UE A and } \\
\text { UE C } \\
\text { receive } \\
\text { GROUP } \\
\text { CALL } \\
\text { ANNOUN } \\
\text { CEMENT }\end{array}$ & $\begin{array}{l}\text { S3: part of } \\
\text { ongoing } \\
\text { call }\end{array}$ & - & - & $\begin{array}{l}\text { UE A checks if } \\
\text { MCPTT Group ID } \\
\text { IE matches stored } \\
\text { MCPTT Group ID } \\
\text { of the call, stops } \\
\text { TFG1 and TFG3, } \\
\text { stores the values } \\
\text { of Session } \\
\text { Description } \\
\text { Protocol (SDP) } \\
\text { IE, Call Identifier } \\
\text { IE, originating } \\
\text { MCPTT user ID } \\
\text { IE, Refresh } \\
\text { interval IE, Call } \\
\text { start time IE of } \\
\text { the GROUP } \\
\text { CALL } \\
\text { ANNOUNCEME } \\
\text { NT as } \\
\text { corresponding } \\
\text { values, establishes } \\
\text { media session, } \\
\text { starts Floor } \\
\text { Control as } \\
\text { terminating Floor } \\
\text { Participant, and } \\
\text { starts TFG2 and } \\
\text { TFG6; } \\
\text { UE C stops } \\
\text { TFG2, starts } \\
\text { TFG2, and sets } \\
\text { the stored probe } \\
\text { response of the } \\
\text { call to "false”. }\end{array}$ & $\begin{array}{l}10.2 .2 .4 .3 .2 \\
10.2 .2 .4 .4 .2\end{array}$ \\
\hline
\end{tabular}

And the corresponding figure is Fig. 11: 


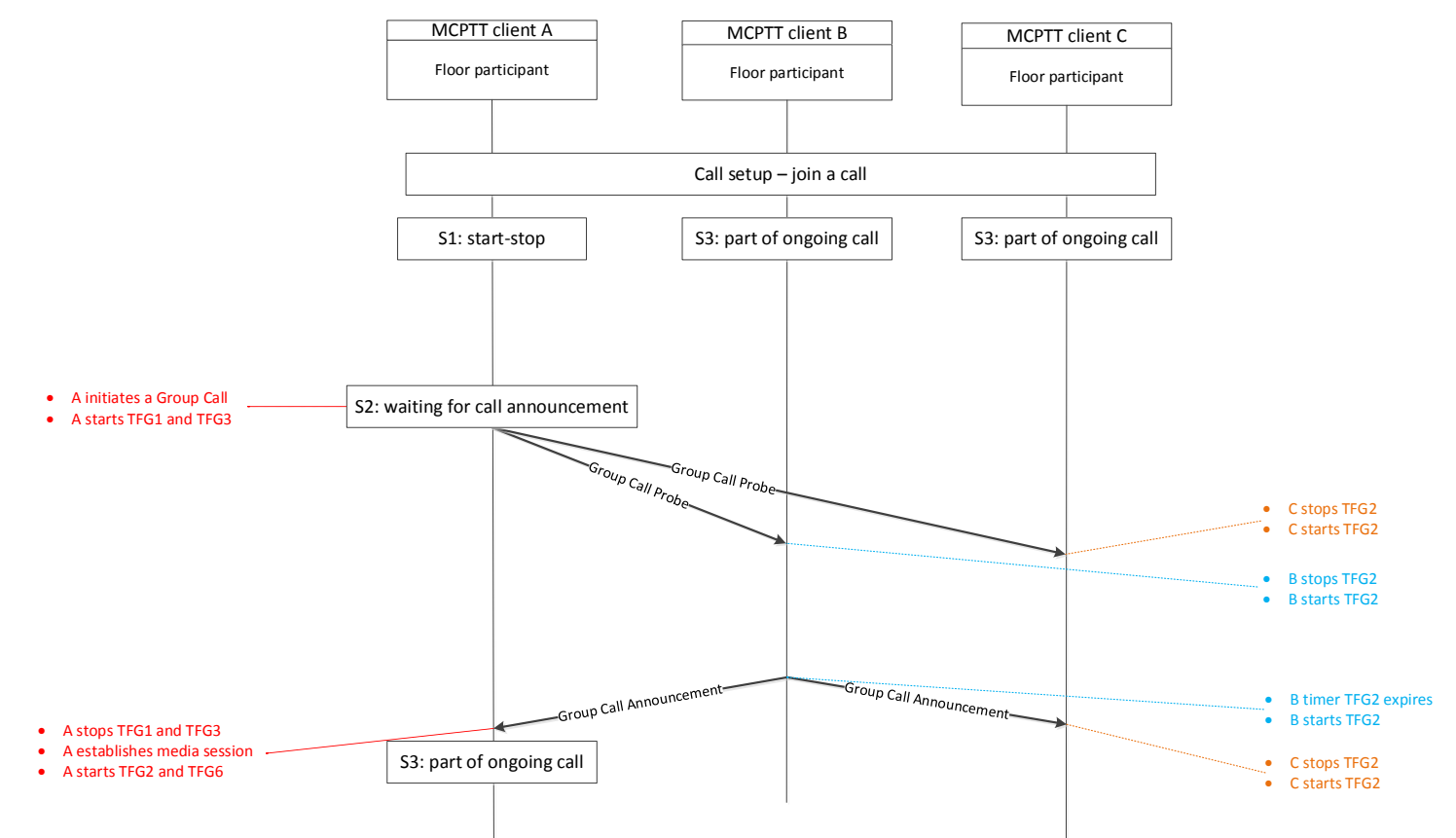

Figure 11: Call setup - join a call

\subsubsection{Call setup - establish a new call with confirm mode indication IE in the Call Announcement message}

In this scenario, a user decides to establish a new group call, and initiates the call as the originating participant. The confirm mode indication IE is included in the Call Announcement message.

Scenario purpose: To observe that a user can establish a new group call with confirm mode indication IE in the Call Announcement message and be joined by other members of the same group.

In this scenario, UE B is configured as "MCPTT User acknowledgement is required upon a terminating call request reception". while UE C is configured as "MCPTT User acknowledgement not required upon a terminating call request reception”.

Table 18: Call setup - establish a new call with confirm mode indication IE in the Call Announcement message

\begin{tabular}{|c|c|c|c|c|c|c|c|}
\hline & Timeline & $\begin{array}{c}\text { Triggering } \\
\text { event }\end{array}$ & $\begin{array}{c}\text { UE A state } \\
\text { transition }\end{array}$ & $\begin{array}{c}\text { UE B state } \\
\text { transition }\end{array}$ & $\begin{array}{c}\text { UE C state } \\
\text { transition }\end{array}$ & $\begin{array}{c}\text { Consequent } \\
\text { behavior }\end{array}$ & $\begin{array}{c}\text { Reference } \\
\text { for details }\end{array}$ \\
\hline 0 & - & - & $\begin{array}{c}\text { S1: start- } \\
\text { stop }\end{array}$ & $\begin{array}{c}\text { S1: start- } \\
\text { stop }\end{array}$ & $\begin{array}{c}\text { S1: start- } \\
\text { stop }\end{array}$ & - & - \\
\hline
\end{tabular}




\begin{tabular}{|c|c|c|c|c|c|c|c|}
\hline & Timeline & $\begin{array}{c}\text { Triggering } \\
\text { event }\end{array}$ & $\begin{array}{l}\text { UE A state } \\
\text { transition }\end{array}$ & $\begin{array}{l}\text { UE B state } \\
\text { transition }\end{array}$ & $\begin{array}{c}\text { UE C state } \\
\text { transition }\end{array}$ & $\begin{array}{c}\text { Consequent } \\
\text { behavior }\end{array}$ & $\begin{array}{l}\text { Reference } \\
\text { for details }\end{array}$ \\
\hline 1 & T0 & $\begin{array}{c}\text { Indication } \\
\text { from UE A } \\
\text { to initiate a } \\
\text { group call }\end{array}$ & $\begin{array}{c}\text { S2: } \\
\text { waiting for } \\
\text { call } \\
\text { announce } \\
\text { ment }\end{array}$ & - & - & $\begin{array}{c}\text { UE A stores } \\
\text { MCPTT group ID } \\
\text { as MCPTT group } \\
\text { ID of the call, } \\
\text { creates a Call type } \\
\text { Control state } \\
\text { machine, } \\
\text { generates and } \\
\text { sends a GROUP } \\
\text { CALL PROBE } \\
\text { message, and } \\
\text { starts TFG1 and } \\
\text { TFG3 } \\
\end{array}$ & 10.2 .2 .4 .2 .1 \\
\hline 2 & $\begin{array}{c}\text { T0 + } \\
\text { TFG3 }\end{array}$ & $\begin{array}{l}\text { UE A } \\
\text { TFG3 } \\
\text { expires }\end{array}$ & - & - & - & $\begin{array}{l}\text { UE A generates } \\
\text { and sends GROUP } \\
\text { CALL PROBE, } \\
\text { and starts TFG3 }\end{array}$ & 10.2 .2 .4 .2 .2 \\
\hline 3 & $\begin{array}{c}\text { T0 + } \\
\text { TFG3 + } \\
\text { t_AB1 }\end{array}$ & $\begin{array}{l}\text { UE B and } \\
\text { UE C } \\
\text { receive } \\
\text { GROUP } \\
\text { CALL } \\
\text { PROBE } \\
\end{array}$ & - & - & - & $\begin{array}{c}\text { UE } \mathbf{B} \text { and } \mathbf{U E} \mathbf{C} \\
\text { discard the } \\
\text { message }\end{array}$ & 10.2 .2 .4 .7 .1 \\
\hline 4 & $\begin{array}{c}\text { T0 + } \\
\text { n*TFG3 }\end{array}$ & $\begin{array}{l}\text { UE A } \\
\text { TFG3 } \\
\text { expires }\end{array}$ & - & - & - & $\begin{array}{c}\text { UE A generates } \\
\text { and sends GROUP } \\
\text { CALL PROBE, } \\
\text { and starts TFG3 }\end{array}$ & 10.2 .2 .4 .2 .2 \\
\hline 5 & $\begin{array}{c}\text { T0 + } \\
\text { nTFG3 + } \\
\text { t_ABn }\end{array}$ & $\begin{array}{l}\text { UE B and } \\
\text { UE C } \\
\text { receive } \\
\text { GROUP } \\
\text { CALL } \\
\text { PROBE }\end{array}$ & - & - & - & $\begin{array}{c}\text { UE } \mathbf{B} \text { and UE C } \\
\text { discard the } \\
\text { message }\end{array}$ & 10.2 .2 .4 .7 .1 \\
\hline
\end{tabular}




\begin{tabular}{|c|c|c|c|c|c|c|c|}
\hline & Timeline & $\begin{array}{c}\text { Triggering } \\
\text { event }\end{array}$ & $\begin{array}{l}\text { UE A state } \\
\text { transition }\end{array}$ & $\begin{array}{l}\text { UE B state } \\
\text { transition }\end{array}$ & $\begin{array}{l}\text { UE C state } \\
\text { transition }\end{array}$ & $\begin{array}{c}\text { Consequent } \\
\text { behavior }\end{array}$ & $\begin{array}{l}\text { Reference } \\
\text { for details }\end{array}$ \\
\hline 6 & $\begin{array}{c}\text { T0 + } \\
\text { TFG1 }\end{array}$ & $\begin{array}{c}\text { UE A } \\
\text { TFG1 } \\
\text { expires }\end{array}$ & $\begin{array}{l}\text { S3: part of } \\
\text { ongoing } \\
\text { call }\end{array}$ & - & - & $\begin{array}{l}\text { UE A stops TFG3, } \\
\text { generates and } \\
\text { stores SDP body, } \\
\text { generates and } \\
\text { stores the call } \\
\text { identifier, selects } \\
\text { and stores refresh } \\
\text { interval, stores its } \\
\text { own MCPTT ID } \\
\text { as originating } \\
\text { MCPTT user ID, } \\
\text { stores current } \\
\text { UTC time as call } \\
\text { start time, } \\
\text { generates and } \\
\text { sends GROUP } \\
\text { CALL } \\
\text { ANNOUNCEME } \\
\text { NT with confirm } \\
\text { mode indication } \\
\text { IE, establishes } \\
\text { media session, } \\
\text { starts Floor } \\
\text { Control as } \\
\text { originating floor } \\
\text { participant, and } \\
\text { starts TFG2 and } \\
\text { TFG6 } \\
\end{array}$ & 10.2 .2 .4 .3 .1 \\
\hline
\end{tabular}




\begin{tabular}{|c|c|c|c|c|c|c|c|}
\hline & Timeline & $\begin{array}{c}\text { Triggering } \\
\text { event }\end{array}$ & $\begin{array}{l}\text { UE A state } \\
\text { transition }\end{array}$ & $\begin{array}{l}\text { UE B state } \\
\text { transition }\end{array}$ & $\begin{array}{l}\text { UE C state } \\
\text { transition }\end{array}$ & $\begin{array}{c}\text { Consequent } \\
\text { behavior }\end{array}$ & $\begin{array}{l}\text { Reference } \\
\text { for details }\end{array}$ \\
\hline 7 & $\begin{array}{c}\text { T0 + } \\
\text { TFG1 + } \\
\text { t_AB(n+ } \\
1)\end{array}$ & $\begin{array}{c}\text { UE B and } \\
\text { UE C } \\
\text { receive } \\
\text { GROUP } \\
\text { CALL } \\
\text { ANNOUN } \\
\text { CEMENT } \\
\text { (contains } \\
\text { Confirm } \\
\text { mode } \\
\text { Indication } \\
\text { IE) }\end{array}$ & - & $\begin{array}{c}\text { S5: } \\
\text { pending } \\
\text { user action } \\
\text { with } \\
\text { confirm } \\
\text { indication } \\
\text { (UE B is } \\
\text { configured } \\
\text { as } \\
\text { “MCPTT } \\
\text { User } \\
\text { acknowled } \\
\text { gement is } \\
\text { required } \\
\text { upon a } \\
\text { terminating } \\
\text { call request } \\
\text { reception”) }\end{array}$ & $\begin{array}{l}\text { S3: part of } \\
\text { ongoing } \\
\text { call } \\
\text { (UE C is } \\
\text { configured } \\
\text { as } \\
\text { "MCPTT } \\
\text { User } \\
\text { acknowled } \\
\text { gement not } \\
\text { required } \\
\text { upon a } \\
\text { terminating } \\
\text { call request } \\
\text { reception") }\end{array}$ & $\begin{array}{l}\text { UE B and UE C } \\
\text { check if MCPTT } \\
\text { group ID IE from } \\
\text { GROUP CALL } \\
\text { ANNOUNCEME } \\
\text { NT does not match } \\
\text { MCPTT group ID } \\
\text { of the call stored } \\
\text { for other state } \\
\text { machines, store } \\
\text { value of SDP IE, } \\
\text { Call Identifier IE, } \\
\text { Originating } \\
\text { MCPTT user ID } \\
\text { IE, Refresh } \\
\text { Interval IE, } \\
\text { MCPTT Group ID } \\
\text { IE, Call start time } \\
\text { IE of the GROUP } \\
\text { CALL } \\
\text { ANNOUNCEME } \\
\text { NT as } \\
\text { corresponding } \\
\text { values, create Call } \\
\text { Type Control state } \\
\text { machine; } \\
\text { UE B starts TFG4; } \\
\text { UE C establishes } \\
\text { media session } \\
\text { based on stored } \\
\text { SDP body of the } \\
\text { call, starts Floor } \\
\text { Control as } \\
\text { terminating floor } \\
\text { participant, } \\
\text { generates and } \\
\text { sends GROUP } \\
\text { CALL ACCEPT } \\
\text { message, and } \\
\text { starts TFG2 and } \\
\text { TFG6 }\end{array}$ & 10.2 .2 .4 .3 .3 \\
\hline
\end{tabular}




\begin{tabular}{|c|c|c|c|c|c|c|c|}
\hline & Timeline & $\begin{array}{c}\text { Triggering } \\
\text { event }\end{array}$ & $\begin{array}{l}\text { UE A state } \\
\text { transition }\end{array}$ & $\begin{array}{l}\text { UE B state } \\
\text { transition }\end{array}$ & $\begin{array}{l}\text { UE C state } \\
\text { transition }\end{array}$ & $\begin{array}{c}\text { Consequent } \\
\text { behavior }\end{array}$ & $\begin{array}{l}\text { Reference } \\
\text { for details }\end{array}$ \\
\hline 8 & $\begin{array}{c}\text { T0 + } \\
\text { TFG1 + } \\
\text { t_AB(n+ } \\
\text { 1) + } \\
\text { t_CA1 }\end{array}$ & $\begin{array}{l}\text { UE A and } \\
\text { UE B } \\
\text { receive } \\
\text { GROUP } \\
\text { CALL } \\
\text { ACCEPT } \\
\text { from UE C }\end{array}$ & - & - & - & $\begin{array}{l}\text { UE A checks if } \\
\text { MCPTT group ID } \\
\text { IE of GROUP } \\
\text { CALL ACCEPT } \\
\text { message matches } \\
\text { stored MCPTT } \\
\text { group ID of the } \\
\text { call, and informs } \\
\text { MCPTT user } \\
\text { about call } \\
\text { acceptance; } \\
\text { UE B discards the } \\
\text { group call control } \\
\text { message } \\
\end{array}$ & $\begin{array}{l}10.2 .2 .4 .3 .6 \\
10.2 .2 .4 .7 .1\end{array}$ \\
\hline 9 & $\begin{array}{c}\text { T0 + } \\
\text { t_B1 } \\
\text { (with } \\
(T F G 1+ \\
t \_A B(n+ \\
1)+ \\
\left.t \_C A 1\right)< \\
\left.t \_B 1\right)\end{array}$ & $\begin{array}{l}\text { UE B } \\
\text { accepts the } \\
\text { terminating } \\
\text { call with } \\
\text { confirm } \\
\text { indication }\end{array}$ & - & $\begin{array}{l}\text { S3: part of } \\
\text { ongoing } \\
\text { call }\end{array}$ & - & $\begin{array}{c}\text { UE B establishes } \\
\text { media session } \\
\text { based on stored } \\
\text { SDP body of the } \\
\text { call, starts Floor } \\
\text { Control as } \\
\text { terminating floor } \\
\text { participant, } \\
\text { generates and } \\
\text { sends GROUP } \\
\text { CALL ACCEPT } \\
\text { message, and } \\
\text { starts TFG2 and } \\
\text { TFG6 } \\
\end{array}$ & 10.2.2.4.3.4 \\
\hline 10 & $\begin{array}{c}\text { T0 + } \\
\text { t_B1 + } \\
\text { t_BA1 }\end{array}$ & $\begin{array}{l}\text { UE A and } \\
\text { UE C } \\
\text { receive } \\
\text { GROUP } \\
\text { CALL } \\
\text { ACCEPT } \\
\text { from UE B }\end{array}$ & - & - & - & $\begin{array}{l}\text { UE A and UE C } \\
\text { check if MCPTT } \\
\text { group ID IE of } \\
\text { GROUP CALL } \\
\text { ACCEPT message } \\
\text { matches stored } \\
\text { MCPTT group ID } \\
\text { of the call, and } \\
\text { inform MCPTT } \\
\text { user about call } \\
\text { acceptance }\end{array}$ & 10.2.2.4.3.6 \\
\hline
\end{tabular}

And the corresponding figure is Fig. 12: 


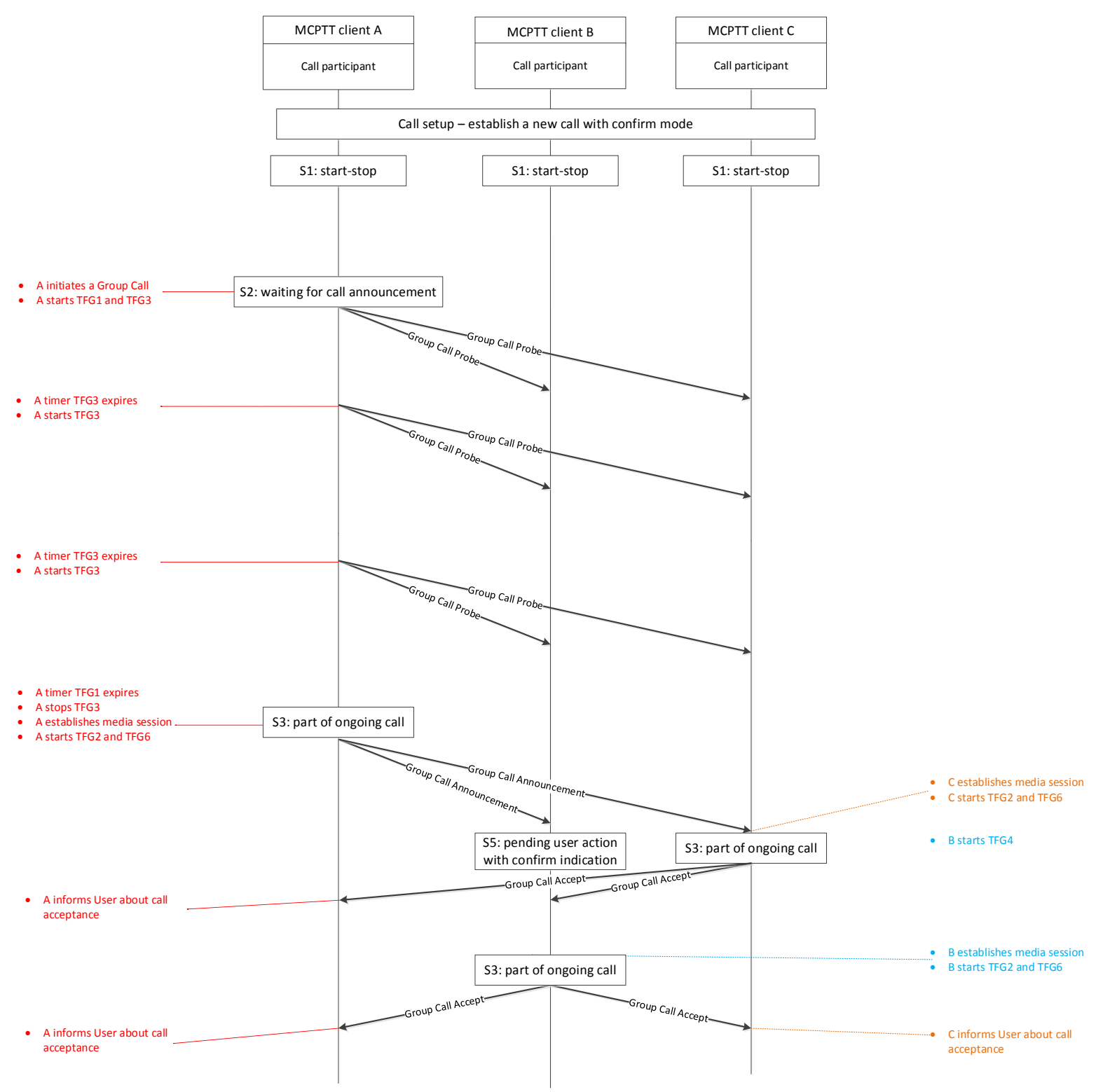

Figure 12: Call setup - establish a new call with confirm mode

\subsubsection{Call setup - establish a new call without confirm mode indication IE in the Call Announcement message}

In this scenario, a user decides to establish a new group call, and initiates the call as the originating participant. The confirm mode indication IE is not included in the Call Announcement message.

Scenario purpose: To observe that a user can establish a new group call without confirm mode indication IE in the Call Announcement message and be joined by other members of the same group. 
In this scenario, UE B is configured as "MCPTT User acknowledgement is required upon a terminating call request reception", while UE C is configured as "MCPTT User acknowledgement not required upon a terminating call request reception”.

Table 19: Call setup - establish a new call without confirm mode indication IE in the Call Announcement message

\begin{tabular}{|c|c|c|c|c|c|c|c|}
\hline & Timeline & $\begin{array}{c}\text { Triggering } \\
\text { event }\end{array}$ & $\begin{array}{l}\text { UE A state } \\
\text { transition }\end{array}$ & $\begin{array}{l}\text { UE B state } \\
\text { transition }\end{array}$ & $\begin{array}{l}\text { UE C state } \\
\text { transition }\end{array}$ & $\begin{array}{c}\text { Consequent } \\
\text { behavior }\end{array}$ & $\begin{array}{l}\text { Reference } \\
\text { for details }\end{array}$ \\
\hline 0 & - & 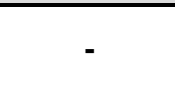 & $\begin{array}{l}\text { S1: start- } \\
\text { stop }\end{array}$ & $\begin{array}{l}\text { S1: start- } \\
\text { stop }\end{array}$ & $\begin{array}{l}\text { S1: start- } \\
\text { stop }\end{array}$ & 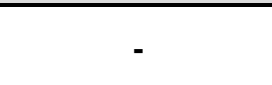 & \\
\hline 1 & T0 & $\begin{array}{c}\text { Indication } \\
\text { from UE A } \\
\text { to initiate a } \\
\text { group call }\end{array}$ & $\begin{array}{c}\text { S2: } \\
\text { waiting for } \\
\text { call } \\
\text { announce } \\
\text { ment }\end{array}$ & - & - & $\begin{array}{c}\text { UE A stores } \\
\text { MCPTT group ID } \\
\text { as MCPTT group } \\
\text { ID of the call, } \\
\text { creates a Call type } \\
\text { Control state } \\
\text { machine, } \\
\text { generates and } \\
\text { sends a GROUP } \\
\text { CALL PROBE } \\
\text { message, and } \\
\text { starts TFG1 and } \\
\text { TFG3 }\end{array}$ & 10.2 .2 .4 .2 .1 \\
\hline 2 & $\begin{array}{c}\text { T0 + } \\
\text { TFG3 }\end{array}$ & $\begin{array}{l}\text { UE A } \\
\text { TFG3 } \\
\text { expires }\end{array}$ & - & - & - & $\begin{array}{l}\text { UE A generates } \\
\text { and sends } \\
\text { GROUP CALL } \\
\text { PROBE, and } \\
\text { starts TFG3 } \\
\end{array}$ & 10.2 .2 .4 .2 .2 \\
\hline 3 & $\begin{array}{c}\text { T0 + } \\
\text { TFG3 + } \\
\text { t_AB1 }\end{array}$ & $\begin{array}{l}\text { UE B and } \\
\text { UE C } \\
\text { receive } \\
\text { GROUP } \\
\text { CALL } \\
\text { PROBE } \\
\end{array}$ & - & - & - & $\begin{array}{c}\text { UE } \mathbf{B} \text { and } \mathbf{U E} \mathbf{C} \\
\text { discard the } \\
\text { message }\end{array}$ & 10.2.2.4.7.1 \\
\hline 4 & $\begin{array}{c}\text { T0 + } \\
\text { n*TFG3 }\end{array}$ & $\begin{array}{l}\text { UE A } \\
\text { TFG3 } \\
\text { expires }\end{array}$ & - & - & - & $\begin{array}{l}\text { UE A generates } \\
\text { and sends } \\
\text { GROUP CALL } \\
\text { PROBE, and } \\
\text { starts TFG3 }\end{array}$ & 10.2 .2 .4 .2 .2 \\
\hline 5 & $\begin{array}{c}\text { T0 + } \\
\text { n*TFG3 } \\
+ \text { t_ABn }\end{array}$ & $\begin{array}{l}\text { UE B and } \\
\text { UE C } \\
\text { receive } \\
\text { GROUP } \\
\text { CALL } \\
\text { PROBE }\end{array}$ & - & - & - & $\begin{array}{c}\text { UE } \mathbf{B} \text { and } \mathbf{U E} \mathbf{C} \\
\text { discard the } \\
\text { message }\end{array}$ & 10.2 .2 .4 .7 .1 \\
\hline
\end{tabular}




\begin{tabular}{|c|c|c|c|c|c|c|c|}
\hline & Timeline & $\begin{array}{c}\text { Triggering } \\
\text { event }\end{array}$ & $\begin{array}{l}\text { UE A state } \\
\text { transition }\end{array}$ & $\begin{array}{l}\text { UE B state } \\
\text { transition }\end{array}$ & $\begin{array}{l}\text { UE C state } \\
\text { transition }\end{array}$ & $\begin{array}{c}\text { Consequent } \\
\text { behavior }\end{array}$ & $\begin{array}{l}\text { Reference } \\
\text { for details }\end{array}$ \\
\hline 6 & $\begin{array}{c}\text { T0 + } \\
\text { TFG1 }\end{array}$ & $\begin{array}{c}\text { UE A } \\
\text { TFG1 } \\
\text { expires }\end{array}$ & $\begin{array}{l}\text { S3: part of } \\
\text { ongoing } \\
\text { call }\end{array}$ & - & - & $\begin{array}{c}\text { UE A stops } \\
\text { TFG3, generates } \\
\text { and stores SDP } \\
\text { body, generates } \\
\text { and stores the call } \\
\text { identifier, selects } \\
\text { and stores refresh } \\
\text { interval, stores its } \\
\text { own MCPTT ID } \\
\text { as originating } \\
\text { MCPTT user ID, } \\
\text { stores current } \\
\text { UTC time as call } \\
\text { start time, } \\
\text { generates and } \\
\text { sends GROUP } \\
\text { CALL } \\
\text { ANNOUNCEME } \\
\text { NT with no } \\
\text { confirm mode } \\
\text { indication IE, } \\
\text { establishes media } \\
\text { session, starts } \\
\text { Floor Control as } \\
\text { originating floor } \\
\text { participant, and } \\
\text { starts TFG2 and } \\
\text { TFG6 }\end{array}$ & 10.2 .2 .4 .3 .1 \\
\hline
\end{tabular}




\begin{tabular}{|c|c|c|c|c|c|c|c|}
\hline & Timeline & $\begin{array}{c}\text { Triggering } \\
\text { event }\end{array}$ & $\begin{array}{l}\text { UE A state } \\
\text { transition }\end{array}$ & $\begin{array}{l}\text { UE B state } \\
\text { transition }\end{array}$ & $\begin{array}{l}\text { UE C state } \\
\text { transition }\end{array}$ & $\begin{array}{c}\text { Consequent } \\
\text { behavior }\end{array}$ & $\begin{array}{l}\text { Reference } \\
\text { for details }\end{array}$ \\
\hline 7 & $\begin{array}{c}\text { T0 + } \\
\text { TFG1 + } \\
\text { t_AB(n+ } \\
1)\end{array}$ & $\begin{array}{c}\text { UE B and } \\
\text { UE C } \\
\text { receive } \\
\text { GROUP } \\
\text { CALL } \\
\text { ANNOUN } \\
\text { CEMENT } \\
\text { (does not } \\
\text { contain } \\
\text { Confirm } \\
\text { mode } \\
\text { Indication } \\
\text { IE) }\end{array}$ & - & $\begin{array}{c}\text { S4: } \\
\text { pending } \\
\text { User } \\
\text { action } \\
\text { without } \\
\text { confirm } \\
\text { indication } \\
\text { (UE B is } \\
\text { configured } \\
\text { as MCPTT } \\
\text { User } \\
\text { acknowled } \\
\text { gement } \\
\text { required } \\
\text { upon a } \\
\text { terminating } \\
\text { call request } \\
\text { reception) }\end{array}$ & $\begin{array}{l}\text { S3: part of } \\
\text { ongoing } \\
\text { call } \\
\text { (UE C is } \\
\text { configured } \\
\text { as MCPTT } \\
\text { User } \\
\text { acknowled } \\
\text { gement not } \\
\text { required } \\
\text { upon a } \\
\text { terminating } \\
\text { call request } \\
\text { reception) }\end{array}$ & $\begin{array}{c}\text { UE B and UE C } \\
\text { check if MCPTT } \\
\text { group ID IE from } \\
\text { GROUP CALL } \\
\text { ANNOUNCEME } \\
\text { NT does not } \\
\text { match MCPTT } \\
\text { group ID of the } \\
\text { call stored for } \\
\text { other state } \\
\text { machines, store } \\
\text { value of SDP IE, } \\
\text { Call Identifier IE, } \\
\text { Originating } \\
\text { MCPTT user ID } \\
\text { IE, Refresh } \\
\text { Interval IE, } \\
\text { MCPTT Group ID } \\
\text { IE, Call start time } \\
\text { IE of the GROUP } \\
\text { CALL } \\
\text { ANNOUNCEME } \\
\text { NT as } \\
\text { corresponding } \\
\text { values, create Call } \\
\text { Type Control state } \\
\text { machine; } \\
\text { UE B starts } \\
\text { TFG4; } \\
\text { UE C establishes } \\
\text { media session } \\
\text { based on stored } \\
\text { SDP body of the } \\
\text { call, starts Floor } \\
\text { Control as } \\
\text { terminating floor } \\
\text { participant, and } \\
\text { starts TFG2 and } \\
\text { TFG6 } \\
\end{array}$ & 10.2 .2 .4 .3 .3 \\
\hline 8 & $\begin{array}{c}\text { T0 + } \\
\text { t_C1 } \\
(\text { with } \\
t \_C 1> \\
T F G 1+ \\
t \_A B(n+ \\
1))\end{array}$ & $\begin{array}{c}\text { UE B } \\
\text { accepts the } \\
\text { terminating } \\
\text { call }\end{array}$ & - & $\begin{array}{l}\text { S3: part of } \\
\text { ongoing } \\
\text { call }\end{array}$ & - & $\begin{array}{c}\text { UE B establishes } \\
\text { media session } \\
\text { based on SDP } \\
\text { body of the call, } \\
\text { starts Floor } \\
\text { Control as } \\
\text { terminating floor } \\
\text { participant, and } \\
\text { starts TFG2 and } \\
\text { TFG6 }\end{array}$ & 10.2 .2 .4 .3 .5 \\
\hline
\end{tabular}

\subsubsection{Call merge}


In this scenario, there is already an ongoing group call with UE A and UE B. Although UE C tries to join the group call by sending a GROUP CALL PROBE, it does not receive a response from UE A or UE B (e.g., temporarily out of range), thus UE C starts a new group call for the same group. Once UE A, UE B and UE C are within transmission range of each other, UE C receives the GROUP CALL ANNOUNCEMENT message from UE A or UE $B$, and since the group IDs match and the starting time of UE C's group call is later than the original group call, UE $\mathrm{C}$ merges with the existing group call. There is no state change for UE A or UE $\mathrm{B}$ during the merging process.

Scenario purpose: To observe that a user, separated from other users already engaged in an ongoing group call, creates his own group call; once this user gets back in range of the other ongoing group call, the newly created group call merges with the older one.

Table 20: Call merge

\begin{tabular}{|c|c|c|c|c|c|c|c|}
\hline & Timeline & $\begin{array}{c}\text { Triggering } \\
\text { event }\end{array}$ & $\begin{array}{l}\text { UE A state } \\
\text { transition }\end{array}$ & $\begin{array}{l}\text { UE B state } \\
\text { transition }\end{array}$ & $\begin{array}{l}\text { UE C state } \\
\text { transition }\end{array}$ & $\begin{array}{c}\text { Consequent } \\
\text { behavior }\end{array}$ & $\begin{array}{l}\text { Reference } \\
\text { for details }\end{array}$ \\
\hline 0 & - & - & $\begin{array}{l}\text { S3: part of } \\
\text { ongoing call } \\
\text { (group } \\
I D=n) \\
\end{array}$ & $\begin{array}{l}\text { S3: part of } \\
\text { ongoing call } \\
\text { (group } \\
I D=n) \\
\end{array}$ & $\begin{array}{l}\text { S1: start- } \\
\text { stop }\end{array}$ & - & - \\
\hline 1 & T0 & $\begin{array}{c}\text { Indication } \\
\text { from UE C } \\
\text { to initiate a } \\
\text { group call } \\
\text { with group } \\
\text { ID=n }\end{array}$ & - & - & $\begin{array}{c}\text { S2: } \\
\text { waiting for } \\
\text { call } \\
\text { announce } \\
\text { ment }\end{array}$ & $\begin{array}{l}\text { UE C stores } \\
\text { MCPTT group ID } \\
\text { as MCPTT group } \\
\text { ID of the call, } \\
\text { creates a Call } \\
\text { Type Control } \\
\text { state machine, } \\
\text { generates and } \\
\text { sends a GROUP } \\
\text { CALL PROBE } \\
\text { message with } \\
\text { MCPTT group ID } \\
=\text { n, and starts } \\
\text { TFG1 and TFG3 } \\
\end{array}$ & 10.2.2.4.2.1 \\
\hline 2 & $\begin{array}{c}\text { T0 + } \\
\text { TFG3 }\end{array}$ & $\begin{array}{c}\text { UE C } \\
\text { TFG3 } \\
\text { expires }\end{array}$ & - & - & - & $\begin{array}{l}\text { UE C generates } \\
\text { and sends } \\
\text { GROUP CALL } \\
\text { PROBE, and } \\
\text { starts TFG3 } \\
\end{array}$ & 10.2.2.4.2.2 \\
\hline 3 & $\begin{array}{c}\mathrm{T} 0+ \\
\mathrm{n} * \mathrm{TFG} 3\end{array}$ & $\begin{array}{c}\text { UE C } \\
\text { TFG3 } \\
\text { expires }\end{array}$ & - & - & - & $\begin{array}{l}\text { UE C generates } \\
\text { and sends } \\
\text { GROUP CALL } \\
\text { PROBE, and } \\
\text { starts TFG3 } \\
\end{array}$ & 10.2 .2 .4 .2 .2 \\
\hline
\end{tabular}




\begin{tabular}{|c|c|c|c|c|c|c|c|}
\hline & Timeline & $\begin{array}{c}\text { Triggering } \\
\text { event }\end{array}$ & $\begin{array}{l}\text { UE A state } \\
\text { transition }\end{array}$ & $\begin{array}{l}\text { UE B state } \\
\text { transition }\end{array}$ & $\begin{array}{c}\text { UE C state } \\
\text { transition }\end{array}$ & $\begin{array}{c}\text { Consequent } \\
\text { behavior }\end{array}$ & $\begin{array}{l}\text { Reference } \\
\text { for details }\end{array}$ \\
\hline 4 & $\begin{array}{c}\text { T0 + } \\
\text { TFG1 }\end{array}$ & $\begin{array}{l}\text { UE C } \\
\text { TFG1 } \\
\text { expires }\end{array}$ & - & - & $\begin{array}{l}\text { S3: part of } \\
\text { ongoing } \\
\text { call (group } \\
I D=n \text {, but } \\
\text { not the } \\
\text { same group } \\
\text { call as } U E \\
\text { A and } U E \\
\text { B) }\end{array}$ & $\begin{array}{l}\text { UE C sets the } \\
\text { originating } \\
\text { MCPTT user ID } \\
\text { of the call to the } \\
\text { ID of UE C, sets } \\
\text { the call identifier, } \\
\text { the SDP body, the } \\
\text { refresh interval, } \\
\text { establishes media } \\
\text { session, starts } \\
\text { floor control as } \\
\text { originating } \\
\text { participant, } \\
\text { generates and } \\
\text { sends GROUP } \\
\text { CALL } \\
\text { ANNOUNCEME } \\
\text { NT, stops TFG3, } \\
\text { and starts TFG2 } \\
\text { and TFG6 }\end{array}$ & 10.2.2.4.3.1 \\
\hline 5 & $\begin{array}{c}\text { T0 + } \\
\text { TFG1 + } \\
\text { TFG2 }\end{array}$ & $\begin{array}{l}\text { UE C } \\
\text { TFG2 } \\
\text { expires }\end{array}$ & - & - & - & $\begin{array}{c}\text { UE C generates } \\
\text { and sends } \\
\text { GROUP CALL } \\
\text { ANNOUNCEME } \\
\text { NT, and starts } \\
\text { TFG2 }\end{array}$ & 10.2.2.4.4.1 \\
\hline 6 & $\begin{array}{c}\text { T0 + } \\
\text { TFG1 + } \\
\text { TFG2 + } \\
\text { t_CA1 }\end{array}$ & $\begin{array}{l}\text { UE A and } \\
\text { UE B } \\
\text { receive } \\
\text { GROUP } \\
\text { CALL } \\
\text { ANNOUN } \\
\text { CEMENT } \\
\text { (from UE C } \\
\text { with } \\
\text { MCPTT } \\
\text { group ID } \\
\text { matching } \\
\text { stored } \\
\text { MCPTT } \\
\text { group ID of } \\
\text { the call) } \\
\end{array}$ & - & - & - & $\begin{array}{l}\text { UE A and UE B } \\
\text { compare the Call } \\
\text { start time IE of } \\
\text { the GROUP } \\
\text { CALL } \\
\text { ANNOUNCEME } \\
\text { NT message from } \\
\text { UE C and } \\
\text { determine it is not } \\
\text { lower than the } \\
\text { stored call start } \\
\text { time of the call } \\
\text { and take no } \\
\text { further action }\end{array}$ & 10.2.2.4.6.1 \\
\hline 7 & $\begin{array}{c}\text { T0 + } \\
\text { t_A1 } \\
\text { (with } \\
t \_A 1> \\
\text { TFG1 + } \\
\text { TFG2 + } \\
\left.t \_C A 1\right)\end{array}$ & $\begin{array}{l}\text { UE A } \\
\text { TFG2 } \\
\text { expires }\end{array}$ & - & - & - & $\begin{array}{c}\text { UE A generates } \\
\text { and sends } \\
\text { GROUP CALL } \\
\text { ANNOUNCEME } \\
\text { NT, and starts } \\
\text { TFG2 }\end{array}$ & 10.2.2.4.4.1 \\
\hline
\end{tabular}




\begin{tabular}{|c|c|c|c|c|c|c|c|}
\hline & Timeline & $\begin{array}{c}\text { Triggering } \\
\text { event }\end{array}$ & $\begin{array}{l}\text { UE A state } \\
\text { transition }\end{array}$ & $\begin{array}{l}\text { UE B state } \\
\text { transition }\end{array}$ & $\begin{array}{l}\text { UE C state } \\
\text { transition }\end{array}$ & $\begin{array}{c}\text { Consequent } \\
\text { behavior }\end{array}$ & $\begin{array}{l}\text { Reference } \\
\text { for details }\end{array}$ \\
\hline 8 & $\begin{array}{c}\mathrm{T} 0+ \\
\mathrm{t} \_\mathrm{A} 1+ \\
\mathrm{t} \_\mathrm{AB} 1\end{array}$ & $\begin{array}{l}\text { UE C and } \\
\text { UE B } \\
\text { receive } \\
\text { GROUP } \\
\text { CALL } \\
\text { ANNOUN } \\
\text { CEMENT } \\
\text { (with } \\
\text { MCPTT } \\
\text { group ID } \\
\text { matching } \\
\text { stored } \\
\text { MCPTT } \\
\text { group ID of } \\
\text { the call) }\end{array}$ & - & - & - & $\begin{array}{c}\text { UE C stores } \\
\text { value of SDP IE, } \\
\text { Call Identifier IE, } \\
\text { Originating } \\
\text { MCPTT user ID } \\
\text { IE, Refresh } \\
\text { Interval IE and } \\
\text { Call start time IE, } \\
\text { adjusts the media } \\
\text { session, stops } \\
\text { TFG6 and TFG2, } \\
\text { and starts again } \\
\text { TFG6 and TFG2; } \\
\text { UE B stops TFG2 } \\
\text { and starts TFG2 }\end{array}$ & $\begin{array}{l}10.2 .2 .4 .6 .1 \\
10.2 .2 .4 .4 .2\end{array}$ \\
\hline
\end{tabular}

And the corresponding figure is Fig. 13: 


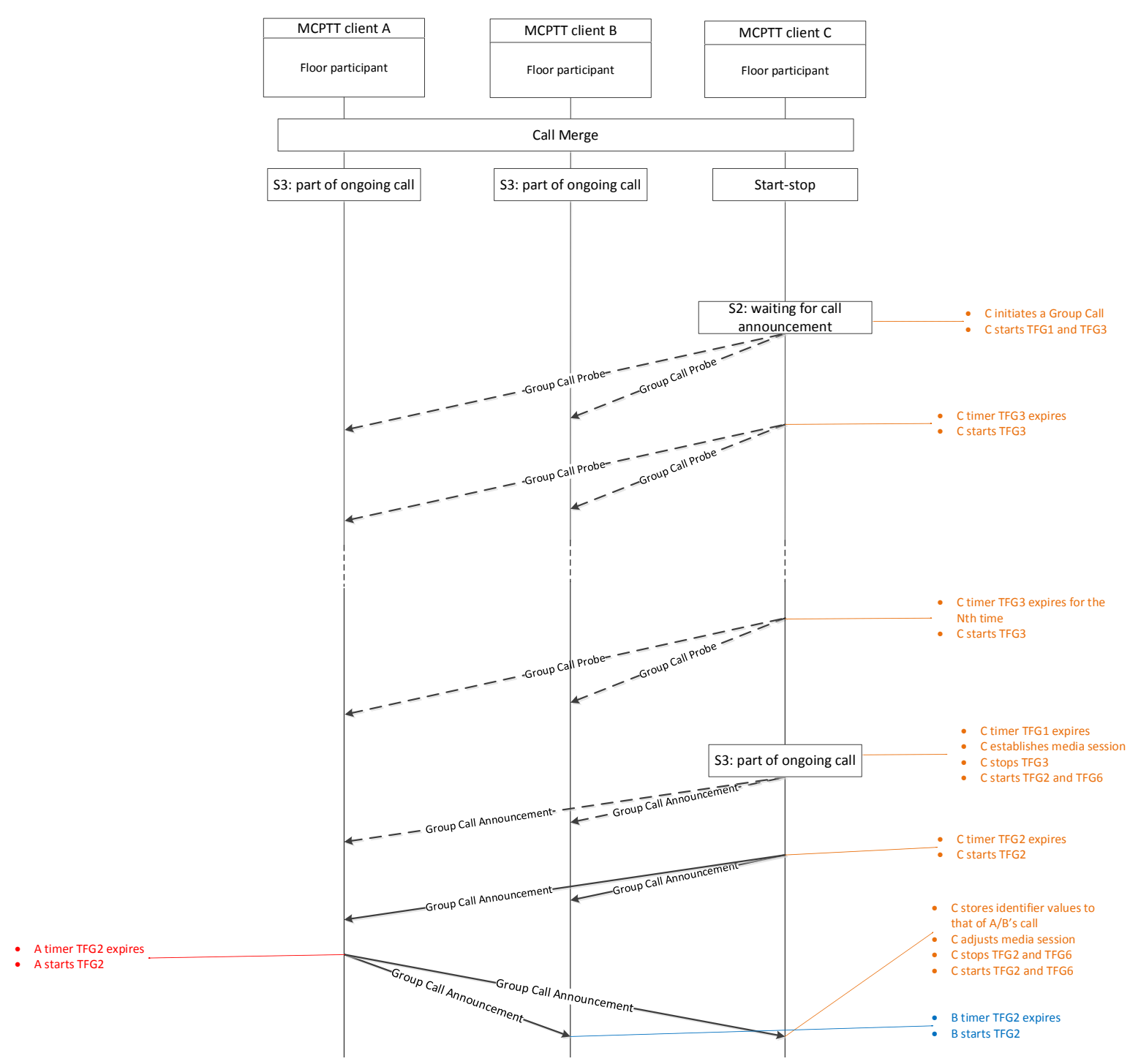

Figure 13: Call merge

\subsubsection{Call release}

\subsubsection{Call release while in the call}

In this scenario, UE A starts in the state, "S3: part of ongoing call”, and decides to withdraw from the call. We assume here that TFG5 < TFG2 for this particular scenario.

Scenario purpose: To observe that a user is able to release from an already ongoing call. 
Table 21: Call release while in the call

\begin{tabular}{|c|c|c|c|c|c|c|c|}
\hline & Timeline & $\begin{array}{c}\text { Triggering } \\
\text { event }\end{array}$ & $\begin{array}{l}\text { UE A state } \\
\text { transition }\end{array}$ & $\begin{array}{l}\text { UE B state } \\
\text { transition }\end{array}$ & $\begin{array}{l}\text { UE C state } \\
\text { transition }\end{array}$ & $\begin{array}{l}\text { Consequent } \\
\text { behavior }\end{array}$ & $\begin{array}{l}\text { Reference } \\
\text { for details }\end{array}$ \\
\hline 0 & - & - & $\begin{array}{l}\text { S3: part of } \\
\text { ongoing call }\end{array}$ & $\begin{array}{l}\text { S3: part of } \\
\text { ongoing call }\end{array}$ & $\begin{array}{l}\text { S3: part of } \\
\text { ongoing call }\end{array}$ & - & - \\
\hline 1 & T0 & $\begin{array}{c}\text { UE A } \\
\text { indication } \\
\text { from } \\
\text { MCPTT } \\
\text { user to } \\
\text { release }\end{array}$ & $\begin{array}{c}\text { S6: } \\
\text { ignoring } \\
\text { incoming } \\
\text { call } \\
\text { announcem } \\
\text { ents }\end{array}$ & - & - & $\begin{array}{c}\text { UE A } \\
\text { releases } \\
\text { media } \\
\text { session, } \\
\text { stops TFG2, } \\
\text { and starts } \\
\text { TFG5 }\end{array}$ & 10.2.2.4.5.1 \\
\hline 2 & $\begin{array}{l}\mathrm{T} 0+ \\
\mathrm{t} \_\mathrm{B} 1\end{array}$ & $\begin{array}{c}\text { UE B } \\
\text { TFG2 } \\
\text { expires }\end{array}$ & - & - & - & $\begin{array}{c}\text { UE B } \\
\text { generates } \\
\text { and sends } \\
\text { GROUP } \\
\text { CALL } \\
\text { ANNOUNC } \\
\text { EMENT, if } \\
\text { stored probe } \\
\text { response } \\
\text { value of the } \\
\text { call is set to } \\
\text { "true", sets } \\
\text { stored probe } \\
\text { response } \\
\text { value of the } \\
\text { call to } \\
\text { "false", and } \\
\text { starts TFG2 }\end{array}$ & 10.2.2.4.4.1 \\
\hline
\end{tabular}




\begin{tabular}{|c|c|c|c|c|c|c|c|}
\hline & Timeline & $\begin{array}{c}\text { Triggering } \\
\text { event }\end{array}$ & $\begin{array}{l}\text { UE A state } \\
\text { transition }\end{array}$ & $\begin{array}{l}\text { UE B state } \\
\text { transition }\end{array}$ & $\begin{array}{l}\text { UE C state } \\
\text { transition }\end{array}$ & $\begin{array}{c}\text { Consequent } \\
\text { behavior }\end{array}$ & $\begin{array}{l}\text { Reference } \\
\text { for details }\end{array}$ \\
\hline 3 & $\begin{array}{c}\text { T0 + } \\
\text { t_B1 + } \\
\text { t_BA1 }\end{array}$ & $\begin{array}{c}\text { UE A and } \\
\text { UE C } \\
\text { receive } \\
\text { GROUP } \\
\text { CALL } \\
\text { ANNOUN } \\
\text { CEMENT }\end{array}$ & - & - & - & $\begin{array}{l}\text { UE A stores } \\
\text { value of } \\
\text { SDP IE, Call } \\
\text { identifier IE, } \\
\text { Originating } \\
\text { MCPTT } \\
\text { user ID IE, } \\
\text { refresh } \\
\text { interval IE, } \\
\text { Call start } \\
\text { time IE of } \\
\text { the GROUP } \\
\text { CALL } \\
\text { ANNOUNC } \\
\text { EMENT as } \\
\text { correspondi } \\
\text { ng values, } \\
\text { stops TFG5, } \\
\text { and starts } \\
\text { TFG5; } \\
\text { UE C stops } \\
\text { TFG2, starts } \\
\text { TFG2 and } \\
\text { sets probe } \\
\text { response of } \\
\text { the call to } \\
\text { "false” }\end{array}$ & $\begin{array}{l}10.2 .2 .4 .5 .2 \\
10.2 .2 .4 .4 .2\end{array}$ \\
\hline$\overline{4}$ & $\begin{array}{c}\text { T0 + } \\
\text { TFG5 }\end{array}$ & $\begin{array}{c}\text { UE A } \\
\text { TFG5 } \\
\text { expires }\end{array}$ & $\begin{array}{l}\text { S1: start- } \\
\text { stop }\end{array}$ & - & - & $\begin{array}{c}\text { UE A } \\
\text { releases } \\
\text { stored SDP } \\
\text { body, call } \\
\text { identifier, } \\
\text { originating } \\
\text { MCPTT } \\
\text { user ID, } \\
\text { refresh } \\
\text { interval, } \\
\text { MCPTT } \\
\text { Group ID, } \\
\text { call start } \\
\text { time of the } \\
\text { call, and } \\
\text { destroys } \\
\text { Call Type } \\
\text { Control state } \\
\text { machine }\end{array}$ & 10.2 .2 .4 .5 .4 \\
\hline
\end{tabular}

\subsubsection{Call release after call probe and return to "S1: start-stop"}

In this scenario, a user in the "S2: waiting for call announcement" state decides to release its probe to join the call, and transitions to "S1: start-stop" state. The user does not receive any Group Call Announcement before transitioning to "S1: start-stop” state. 
Scenario purpose: To observe that a user can release its probe to find an ongoing call and returns to "S1: start-stop" state.

Table 22: Call release after call probe and return to "S1: start-stop"

\begin{tabular}{|c|c|c|c|c|c|c|c|}
\hline & Timeline & $\begin{array}{c}\text { Triggering } \\
\text { event }\end{array}$ & $\begin{array}{l}\text { UE A state } \\
\text { transition }\end{array}$ & $\begin{array}{l}\text { UE B state } \\
\text { transition }\end{array}$ & $\begin{array}{c}\text { UE C state } \\
\text { transition }\end{array}$ & $\begin{array}{c}\text { Consequent } \\
\text { behavior }\end{array}$ & $\begin{array}{l}\text { Reference } \\
\text { for details } \\
\end{array}$ \\
\hline 0 & - & - & S1: start-stop & $\begin{array}{l}\text { S3: part of } \\
\text { ongoing call }\end{array}$ & $\begin{array}{l}\text { S3: part of } \\
\text { ongoing call }\end{array}$ & - & - \\
\hline 1 & T0 & $\begin{array}{c}\text { Indication } \\
\text { from UE A } \\
\text { to join a } \\
\text { group call }\end{array}$ & $\begin{array}{l}\text { S2: waiting for } \\
\text { call } \\
\text { announcement }\end{array}$ & - & - & $\begin{array}{l}\text { UE A stores } \\
\text { MCPTT } \\
\text { group ID as } \\
\text { MCPTT } \\
\text { group ID of } \\
\text { the call, } \\
\text { creates a } \\
\text { Call Type } \\
\text { Control } \\
\text { state } \\
\text { machine, } \\
\text { generates } \\
\text { and sends a } \\
\text { GROUP } \\
\text { CALL } \\
\text { PROBE } \\
\text { message, } \\
\text { and starts } \\
\text { TFG1 and } \\
\text { TFG3 }\end{array}$ & 10.2.2.4.2.1 \\
\hline 2 & $\begin{array}{l}\mathrm{T} 0+ \\
\mathrm{t} \_\mathrm{AB} 1\end{array}$ & $\begin{array}{l}\mathbf{U E} \mathbf{B} \text { and } \\
\text { UE } \mathbf{C} \\
\text { receive } \\
\text { GROUP } \\
\text { CALL } \\
\text { PROBE }\end{array}$ & - & - & - & $\begin{array}{c}\text { UE B and } \\
\text { UE C check } \\
\text { if MCPTT } \\
\text { group ID IE } \\
\text { matches } \\
\text { stored } \\
\text { MCPTT } \\
\text { Group ID of } \\
\text { the call, } \\
\text { stop TFG2, } \\
\text { start TFG2, } \\
\text { and set } \\
\text { stored probe } \\
\text { response of } \\
\text { the call to } \\
\text { "true" }\end{array}$ & 10.2.2.4.2.3 \\
\hline 3 & $\begin{array}{l}\text { T0 + } \\
\text { t_A1 }\end{array}$ & $\begin{array}{c}\text { UE A } \\
\text { indication } \\
\text { from } \\
\text { MCPTT } \\
\text { User to } \\
\text { release the } \\
\text { Group Call }\end{array}$ & $\begin{array}{l}\text { S7: waiting for } \\
\text { call } \\
\text { announcement } \\
\text { after call } \\
\text { release }\end{array}$ & - & - & $\begin{array}{c}\text { UE A stops } \\
\text { TFG3 }\end{array}$ & 10.2 .2 .4 .5 .5 \\
\hline
\end{tabular}




\begin{tabular}{|c|c|c|c|c|c|c|c|}
\hline & Timeline & $\begin{array}{c}\text { Triggering } \\
\text { event }\end{array}$ & $\begin{array}{l}\text { UE A state } \\
\text { transition }\end{array}$ & $\begin{array}{l}\text { UE B state } \\
\text { transition }\end{array}$ & $\begin{array}{l}\text { UE C state } \\
\text { transition }\end{array}$ & $\begin{array}{c}\text { Consequent } \\
\text { behavior }\end{array}$ & $\begin{array}{l}\text { Reference } \\
\text { for details }\end{array}$ \\
\hline 4 & $\begin{array}{c}\text { T0 + } \\
\text { TFG1 } \\
\text { (with } \\
\text { TFG1< } \\
\left(T \_A B 1+\right. \\
\text { TFG2)) }\end{array}$ & $\begin{array}{c}\text { UE A } \\
\text { TFG1 } \\
\text { expires }\end{array}$ & S1: start-stop & - & - & $\begin{array}{c}\text { UE A } \\
\text { releases } \\
\text { stored } \\
\text { MCPTT } \\
\text { Group ID of } \\
\text { the call, and } \\
\text { destroys } \\
\text { Call Type } \\
\text { Control } \\
\text { state } \\
\text { machine }\end{array}$ & 10.2.2.4.5.8 \\
\hline
\end{tabular}

\subsubsection{Call release after call probe and enter " $\mathrm{S6}$ : ignoring incoming call announcements"}

In this scenario, a user in the "S2: waiting for call announcement" state decides to release its probe to join the call and receives a Group Call Announcement before it can transition to "S1: start-stop” state. Thus, it enters "S6: ignoring incoming call announcements” instead of S1.

Scenario purpose: To observe that a user can release its probe to find an ongoing call and enters "S6: ignoring incoming call announcements" state.

Table 23: Call release after call probe and enter "S6: ignoring incoming call announcements"

\begin{tabular}{|c|c|c|c|c|c|c|c|}
\hline & Timeline & $\begin{array}{c}\text { Triggering } \\
\text { event }\end{array}$ & $\begin{array}{l}\text { UE A state } \\
\text { transition }\end{array}$ & $\begin{array}{l}\text { UE B state } \\
\text { transition }\end{array}$ & $\begin{array}{l}\text { UE C state } \\
\text { transition }\end{array}$ & $\begin{array}{c}\text { Consequent } \\
\text { behavior }\end{array}$ & $\begin{array}{l}\text { Reference } \\
\text { for details }\end{array}$ \\
\hline 0 & - & - & $\begin{array}{l}\text { S1: start- } \\
\text { stop }\end{array}$ & $\begin{array}{l}\text { S3: part of } \\
\text { ongoing } \\
\text { call }\end{array}$ & $\begin{array}{c}\text { S3: part of } \\
\text { ongoing } \\
\text { call }\end{array}$ & - & - \\
\hline 1 & T0 & $\begin{array}{c}\text { Indication } \\
\text { from UE A } \\
\text { to join a } \\
\text { group call }\end{array}$ & $\begin{array}{c}\text { S2: } \\
\text { waiting for } \\
\text { call } \\
\text { announce } \\
\text { ment }\end{array}$ & - & - & $\begin{array}{c}\text { UE A stores } \\
\text { MCPTT group ID as } \\
\text { MCPTT group ID of } \\
\text { the call, creates a } \\
\text { Call Type Control } \\
\text { state machine, } \\
\text { generates and sends } \\
\text { a GROUP CALL } \\
\text { PROBE message, } \\
\text { and starts TFG1 and } \\
\text { TFG3 } \\
\end{array}$ & 10.2.2.4.2.1 \\
\hline
\end{tabular}




\begin{tabular}{|c|c|c|c|c|c|c|c|}
\hline & Timeline & $\begin{array}{c}\text { Triggering } \\
\text { event }\end{array}$ & $\begin{array}{l}\text { UE A state } \\
\text { transition }\end{array}$ & $\begin{array}{l}\text { UE B state } \\
\text { transition }\end{array}$ & $\begin{array}{l}\text { UE C state } \\
\text { transition }\end{array}$ & $\begin{array}{c}\text { Consequent } \\
\text { behavior }\end{array}$ & $\begin{array}{l}\text { Reference } \\
\text { for details }\end{array}$ \\
\hline 2 & $\begin{array}{l}\mathrm{T} 0+ \\
\mathrm{t} \_\mathrm{AB} 1\end{array}$ & $\begin{array}{l}\text { UE B and } \\
\text { UE C } \\
\text { receive } \\
\text { GROUP } \\
\text { CALL } \\
\text { PROBE }\end{array}$ & - & - & - & $\begin{array}{c}\text { UE B and UE C } \\
\text { check if MCPTT } \\
\text { group ID IE matches } \\
\text { stored MCPTT } \\
\text { Group ID of the call, } \\
\text { stop TFG2, start } \\
\text { TFG2, and set } \\
\text { stored probe } \\
\text { response of the call } \\
\text { to "true" } \\
\end{array}$ & 10.2.2.4.2.3 \\
\hline 3 & T0 + t_A1 & $\begin{array}{c}\text { UE A } \\
\text { indication } \\
\text { from } \\
\text { MCPTT } \\
\text { User to } \\
\text { release the } \\
\text { Group Call } \\
\end{array}$ & $\begin{array}{l}\text { S7: } \\
\text { waiting for } \\
\quad \text { call } \\
\text { announce } \\
\text { ment after } \\
\text { call release }\end{array}$ & - & - & UE A stops TFG3 & 10.2.2.4.5.5 \\
\hline 4 & $\begin{array}{c}\text { T0 + } \\
\text { t_AB1 + } \\
\text { TFG2 } \\
(\text { with } \\
\left(t \_A B 1+\right. \\
\text { TFG2) } \\
<\text { TFG1) } \\
\end{array}$ & $\begin{array}{c}\text { UE C } \\
\text { TFG2 } \\
\text { expires }\end{array}$ & - & - & - & $\begin{array}{c}\text { UE C generates and } \\
\text { sends GROUP } \\
\text { CALL } \\
\text { ANNOUNCEMEN } \\
\text { T, and starts TFG2 }\end{array}$ & 10.2.2.4.4.1 \\
\hline 5 & $\begin{array}{c}\mathrm{T} 0+ \\
\mathrm{t} \_\mathrm{AB} 1+ \\
\mathrm{TFG} 2+ \\
\mathrm{t} \_\mathrm{CA} 1\end{array}$ & $\begin{array}{l}\text { UE } \mathbf{A} \text { and } \\
\text { UE } \mathbf{B} \\
\text { receive } \\
\text { GROUP } \\
\text { CALL } \\
\text { ANNOUN } \\
\text { CEMENT }\end{array}$ & $\begin{array}{c}\text { S6: } \\
\text { ignoring } \\
\text { incoming } \\
\text { call } \\
\text { announce } \\
\text { ments }\end{array}$ & - & - & $\begin{array}{l}\text { UE A stores values } \\
\text { of SDP IE, Call } \\
\text { Identifier IE, } \\
\text { Originating MCPTT } \\
\text { user ID IE, Refresh } \\
\text { interval IE and Call } \\
\text { Start Time IE of the } \\
\text { GROUP CALL } \\
\text { ANNOUNCEMEN } \\
\text { T message as } \\
\text { corresponding } \\
\text { values, stops TFG1, } \\
\text { and starts TFG5; } \\
\text { UE B stops TFG2 } \\
\text { and starts again } \\
\text { TFG2 }\end{array}$ & $\begin{array}{l}10.2 .2 .4 .5 .7 \\
10.2 .2 .4 .4 .2\end{array}$ \\
\hline
\end{tabular}

\subsubsection{Call release while pending user action - without confirm indication}

In this scenario, a user in the "S4: pending user action without confirmation indication" state decides to release the call. In this scenario, instead of going back to the "S1: start-stop" state, the user decides to initiate a group call again before TFG5 expires, although the user (UE A) released the call before. 
Scenario purpose: To observe that after receiving notification (GROUP CALL ANNOUNCEMENT) of an ongoing call, a UE (without confirmation mode) can release its request to join a call, only to re-enable it later and effectively join the call.

Table 24: Call release while pending user action - without confirm indication

\begin{tabular}{|c|c|c|c|c|c|c|c|}
\hline & Timeline & $\begin{array}{c}\text { Triggering } \\
\text { event }\end{array}$ & $\begin{array}{c}\text { UE A state } \\
\text { transition }\end{array}$ & $\begin{array}{c}\text { UE B state } \\
\text { transition }\end{array}$ & $\begin{array}{c}\text { UE C state } \\
\text { transition }\end{array}$ & $\begin{array}{c}\begin{array}{c}\text { Consequent } \\
\text { behavior }\end{array} \\
\end{array}$ & $\begin{array}{l}\text { Reference } \\
\text { for details }\end{array}$ \\
\hline 0 & - & - & $\begin{array}{l}\text { S4: pending } \\
\text { user action } \\
\text { without } \\
\text { confirm } \\
\text { indication } \\
\end{array}$ & $\begin{array}{l}\text { S3: part of } \\
\text { ongoing call }\end{array}$ & $\begin{array}{l}\text { S3: part of } \\
\text { ongoing call }\end{array}$ & - & - \\
\hline 1 & T0 & $\begin{array}{c}\text { UE A } \\
\text { indication } \\
\text { from } \\
\text { MCPTT } \\
\text { user to } \\
\text { release the } \\
\text { call }\end{array}$ & $\begin{array}{c}\text { S6: } \\
\text { ignoring } \\
\text { incoming } \\
\text { call } \\
\text { announcem } \\
\text { ents }\end{array}$ & - & - & $\begin{array}{c}\text { UE A } \\
\text { releases } \\
\text { media } \\
\text { session, } \\
\text { stops TFG2 } \\
\text { and TFG4, } \\
\text { and starts } \\
\text { TFG5 }\end{array}$ & 10.2 .2 .4 .5 .1 \\
\hline 2 & $\mathrm{~T} 0+\mathrm{t} \_\mathrm{A} 1$ & $\begin{array}{c}\text { UE A } \\
\text { indication } \\
\text { from } \\
\text { MCPTT } \\
\text { user to } \\
\text { initiate a } \\
\text { Group Call } \\
\text { for an } \\
\text { MCPTT } \\
\text { group ID } \\
\text { matching } \\
\text { stored } \\
\text { MCPTT } \\
\text { Group ID } \\
\text { of the call }\end{array}$ & $\begin{array}{l}\text { S3: part of } \\
\text { ongoing } \\
\text { call }\end{array}$ & - & - & $\begin{array}{l}\text { UE A stops } \\
\text { TFG5, } \\
\text { establishes } \\
\text { media } \\
\text { session, } \\
\text { starts Floor } \\
\text { Control as } \\
\text { terminating } \\
\text { Floor } \\
\text { Participant, } \\
\text { and starts } \\
\text { TFG2 and } \\
\text { TFG6 }\end{array}$ & 10.2 .2 .4 .5 .3 \\
\hline
\end{tabular}

\subsubsection{Call release while pending user action - with confirm indication}

In this scenario, a user in the "S5: pending user action with confirmation indication" state decides to release its request to join.

Scenario purpose: To observe that UE A does not respond to the request to join the ongoing call and ignores future GROUP CALL ANNOUNCEMENT messages until the call is terminated or the UE moves out of range so that GROUP CALL ANNOUNCEMENT messages cease to be received by UE A and permits TFG5 to timeout, thus returning UE A to the S1: start-stop state. 
Table 25: Call release while pending user action - with confirm indication

\begin{tabular}{|c|c|c|c|c|c|c|c|}
\hline & Timeline & $\begin{array}{l}\text { Triggering } \\
\text { event }\end{array}$ & $\begin{array}{l}\text { UE A state } \\
\text { transition }\end{array}$ & $\begin{array}{l}\text { UE B state } \\
\text { transition }\end{array}$ & $\begin{array}{c}\text { UE C state } \\
\text { transition }\end{array}$ & $\begin{array}{c}\text { Consequent } \\
\text { behavior }\end{array}$ & $\begin{array}{l}\text { Reference } \\
\text { for details }\end{array}$ \\
\hline 0 & - & - & $\begin{array}{l}\text { S5: pending } \\
\text { user action } \\
\text { with confirm } \\
\text { indication }\end{array}$ & $\begin{array}{l}\text { S3: part of } \\
\text { ongoing call }\end{array}$ & $\begin{array}{l}\text { S3: part of } \\
\text { ongoing call }\end{array}$ & - & 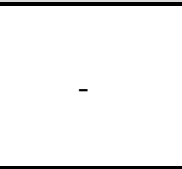 \\
\hline 1 & T0 & $\begin{array}{c}\text { UE A } \\
\text { indication } \\
\text { from } \\
\text { MCPTT } \\
\text { user to } \\
\text { release the } \\
\text { call }\end{array}$ & $\begin{array}{l}\text { S6: ignoring } \\
\text { incoming } \\
\text { call } \\
\text { announceme } \\
\text { nts }\end{array}$ & - & - & $\begin{array}{l}\text { UE A stops } \\
\text { TFG4, and } \\
\text { starts TFG5 }\end{array}$ & 10.2.2.4.5.1 \\
\hline 2 & $\begin{array}{l}\text { T0 + } \\
\text { t_B1 }\end{array}$ & $\begin{array}{c}\text { UE B } \\
\text { TFG2 } \\
\text { expires }\end{array}$ & - & - & - & $\begin{array}{c}\text { UE B } \\
\text { generates } \\
\text { and sends } \\
\text { GROUP } \\
\text { CALL } \\
\text { ANNOUNC } \\
\text { EMENT, if } \\
\text { stored probe } \\
\text { response } \\
\text { value of the } \\
\text { call is set to } \\
\text { "true", sets } \\
\text { stored probe } \\
\text { response } \\
\text { value of the } \\
\text { call to } \\
\text { "false", and } \\
\text { starts TFG2 }\end{array}$ & 10.2.2.4.4.1 \\
\hline
\end{tabular}




\begin{tabular}{|c|c|c|c|c|c|c|c|}
\hline & Timeline & $\begin{array}{c}\text { Triggering } \\
\text { event }\end{array}$ & $\begin{array}{l}\text { UE A state } \\
\text { transition }\end{array}$ & $\begin{array}{l}\text { UE B state } \\
\text { transition }\end{array}$ & $\begin{array}{l}\text { UE C state } \\
\text { transition }\end{array}$ & $\begin{array}{c}\begin{array}{c}\text { Consequent } \\
\text { behavior }\end{array} \\
\end{array}$ & $\begin{array}{l}\text { Reference } \\
\text { for details }\end{array}$ \\
\hline 3 & $\begin{array}{c}\text { T0 + } \\
\text { t_B1 + } \\
\text { t_BA1 }\end{array}$ & $\begin{array}{l}\text { UE } \mathbf{A} \text { and } \\
\text { UE C } \\
\text { receive } \\
\text { GROUP } \\
\text { CALL } \\
\text { ANNOUN } \\
\text { CEMENT }\end{array}$ & - & - & - & $\begin{array}{l}\text { UE A stores } \\
\text { value of } \\
\text { SDP IE, } \\
\text { Call } \\
\text { identifier IE, } \\
\text { Originating } \\
\text { MCPTT } \\
\text { user ID IE, } \\
\text { refresh } \\
\text { interval IE, } \\
\text { Call start } \\
\text { time IE of } \\
\text { the GROUP } \\
\text { CALL } \\
\text { ANNOUNC } \\
\text { EMENT, } \\
\text { stops TFG5, } \\
\text { and starts } \\
\text { TFG5; } \\
\text { UE C stops } \\
\text { TFG2, starts } \\
\text { TFG2, and } \\
\text { sets probe } \\
\text { response of } \\
\text { the call to } \\
\text { "false” }\end{array}$ & $\begin{array}{l}10.2 .2 .4 .5 .2 \\
10.2 .2 .4 .4 .2\end{array}$ \\
\hline 4 & $\begin{array}{c}\text { T0 + } \\
\text { TFG5 }\end{array}$ & $\begin{array}{c}\text { UE A } \\
\text { TFG5 } \\
\text { expires }\end{array}$ & $\begin{array}{l}\text { S1: start- } \\
\text { stop }\end{array}$ & - & - & $\begin{array}{c}\text { UE A } \\
\text { releases } \\
\text { stored SDP } \\
\text { body, call } \\
\text { identifier, } \\
\text { originating } \\
\text { MCPTT } \\
\text { user ID, } \\
\text { refresh } \\
\text { interval, } \\
\text { MCPTT } \\
\text { Group ID, } \\
\text { call start } \\
\text { time of the } \\
\text { call, and } \\
\text { destroys } \\
\text { Call Type } \\
\text { Control state } \\
\text { machine } \\
\end{array}$ & 10.2 .2 .4 .5 .4 \\
\hline
\end{tabular}

\subsubsection{Call release when maximum duration of the call is reached}

In this scenario, one of the participants withdraws from the call because the maximum duration (TFG6) of the call is reached. UE A is then afterwards considered out of range of UE B and $\mathrm{UE} \mathrm{C}$, and is unable to receive their Group Call Announcement messages. 
Scenario purpose: To observe that when the maximum duration of a call occurs for a user, that user withdraws from the call; to observe that this same user, now considered out of range from the other users, goes back to S1: start-stop state of the state machine.

Table 26: Call release when maximum duration of the call is reached

\begin{tabular}{|c|c|c|c|c|c|c|c|}
\hline & Timeline & $\begin{array}{c}\text { Triggering } \\
\text { event }\end{array}$ & $\begin{array}{l}\text { UE A state } \\
\text { transition }\end{array}$ & $\begin{array}{l}\text { UE B state } \\
\text { transition }\end{array}$ & $\begin{array}{l}\text { UE C state } \\
\text { transition }\end{array}$ & $\begin{array}{c}\text { Consequent } \\
\text { behavior }\end{array}$ & $\begin{array}{l}\text { Reference } \\
\text { for details }\end{array}$ \\
\hline 0 & - & - & $\begin{array}{c}\text { S3: part of } \\
\text { ongoing } \\
\text { call }\end{array}$ & $\begin{array}{c}\begin{array}{c}\text { S3: part of } \\
\text { ongoing } \\
\text { call }\end{array} \\
\end{array}$ & $\begin{array}{c}\text { S3: part of } \\
\text { ongoing } \\
\text { call }\end{array}$ & - & - \\
\hline 1 & T0 & $\begin{array}{c}\text { UE A } \\
\text { TFG6 } \\
\text { expires }\end{array}$ & $\begin{array}{c}\text { S6: } \\
\text { ignoring } \\
\text { incoming } \\
\text { call } \\
\text { announce } \\
\text { ments }\end{array}$ & - & - & $\begin{array}{l}\text { UE A releases the } \\
\text { media session, } \\
\text { stops TFG2, and } \\
\text { starts TFG5 }\end{array}$ & 10.2.2.4.5.9 \\
\hline 2 & $\begin{array}{c}\text { T0 + } \\
\text { TFG5 }\end{array}$ & $\begin{array}{l}\text { UE A } \\
\text { TFG5 } \\
\text { expires }\end{array}$ & $\begin{array}{l}\text { S1: start- } \\
\text { stop }\end{array}$ & - & - & $\begin{array}{l}\text { UE A releases } \\
\text { stored SDP body, } \\
\text { call identifier, } \\
\text { originating } \\
\text { MCPTT user ID, } \\
\text { refresh interval, } \\
\text { MCPTT Group ID, } \\
\text { call start time of } \\
\text { the call, and } \\
\text { destroys Call Type } \\
\text { Control state } \\
\text { machine }\end{array}$ & 10.2 .2 .4 .5 .4 \\
\hline
\end{tabular}

\subsubsection{Call release and setup}

In this scenario, after sending a GROUP CALL PROBE message, a user decides to release the call, but later wants to start a new group call with the same group.

Scenario purpose: To observe that a user undecidedly tries to initiate a group call, release such initialization, then initiates it again with no other ongoing call having already been established.

Table 27: Call release and setup

\begin{tabular}{|c|c|c|c|c|c|c|c|}
\hline & Timeline & $\begin{array}{c}\text { Triggering } \\
\text { event }\end{array}$ & $\begin{array}{c}\text { UE A state } \\
\text { transition }\end{array}$ & $\begin{array}{c}\text { UE B state } \\
\text { transition }\end{array}$ & $\begin{array}{c}\text { UE C state } \\
\text { transition }\end{array}$ & $\begin{array}{c}\text { Consequent } \\
\text { behavior }\end{array}$ & $\begin{array}{c}\text { Reference } \\
\text { for details }\end{array}$ \\
\hline 0 & - & - & $\begin{array}{c}\text { S1: start- } \\
\text { stop }\end{array}$ & $\begin{array}{c}\text { S1: start- } \\
\text { stop }\end{array}$ & $\begin{array}{c}\text { S1: start- } \\
\text { stop }\end{array}$ & - & - \\
\hline
\end{tabular}




\begin{tabular}{|c|c|c|c|c|c|c|c|}
\hline & Timeline & $\begin{array}{c}\text { Triggering } \\
\text { event }\end{array}$ & $\begin{array}{l}\text { UE A state } \\
\text { transition }\end{array}$ & $\begin{array}{l}\text { UE B state } \\
\text { transition }\end{array}$ & $\begin{array}{c}\text { UE C state } \\
\text { transition }\end{array}$ & $\begin{array}{c}\begin{array}{c}\text { Consequent } \\
\text { behavior }\end{array} \\
\end{array}$ & $\begin{array}{l}\text { Reference } \\
\text { for details }\end{array}$ \\
\hline 1 & T0 & $\begin{array}{c}\text { Indication } \\
\text { from UE A to } \\
\text { initiate a group } \\
\text { call }\end{array}$ & $\begin{array}{c}\text { S2: waiting } \\
\text { for call } \\
\text { announceme } \\
\text { nt }\end{array}$ & 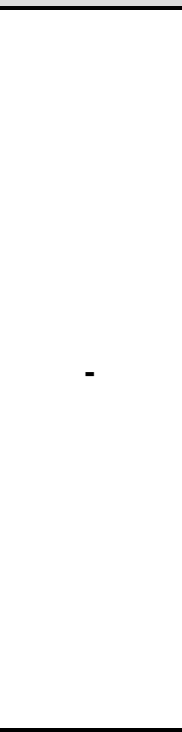 & 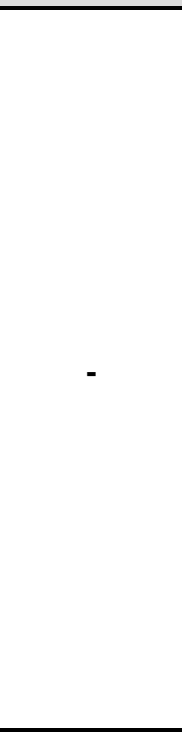 & $\begin{array}{l}\text { UE A stores } \\
\text { MCPTT } \\
\text { group ID as } \\
\text { MCPTT } \\
\text { group ID of } \\
\text { the call, } \\
\text { creates a Call } \\
\text { type Control } \\
\text { state } \\
\text { machine, } \\
\text { generates and } \\
\text { sends a } \\
\text { GROUP } \\
\text { CALL } \\
\text { PROBE } \\
\text { message, and } \\
\text { starts TFG1 } \\
\text { and TFG3 } \\
\end{array}$ & 10.2.2.4.2.1 \\
\hline 2 & $\begin{array}{l}\mathrm{T} 0+ \\
\mathrm{t} \_\mathrm{AB} 1\end{array}$ & $\begin{array}{l}\text { UE } \mathbf{B} \text { and } \mathbf{U E} \\
\mathbf{C} \text { receive } \\
\text { GROUP } \\
\text { CALL PROBE }\end{array}$ & - & - & - & $\begin{array}{c}\mathbf{U E} \mathbf{B} \text { and } \\
\mathbf{U E} \mathbf{C} \text { discard } \\
\text { the call } \\
\text { control } \\
\text { message } \\
\end{array}$ & 10.2.2.4.7.1 \\
\hline 3 & $\begin{array}{l}\text { T0 + } \\
\text { t_A1 }\end{array}$ & $\begin{array}{l}\text { Indication } \\
\text { from UE A to } \\
\text { release the } \\
\text { group call }\end{array}$ & $\begin{array}{c}\text { S7: waiting } \\
\text { for call } \\
\text { announceme } \\
\text { nt after call } \\
\text { release } \\
\end{array}$ & - & - & $\begin{array}{l}\text { UE A stops } \\
\text { TFG3 }\end{array}$ & 10.2 .2 .4 .5 .5 \\
\hline 4 & $\begin{array}{l}\text { T0 + } \\
\mathrm{t} \_ \text {A2 } \\
(\text { with } \\
t \_A 2> \\
\left.t \_A 1\right)\end{array}$ & $\begin{array}{c}\text { Indication } \\
\text { from UE A to } \\
\text { initiate a group } \\
\text { call for } \\
\text { MCPTT group } \\
\text { ID matching } \\
\text { stored MCPTT } \\
\text { group ID of } \\
\text { the call } \\
\end{array}$ & $\begin{array}{c}\text { S2: waiting } \\
\text { for call } \\
\text { announceme } \\
\text { nt }\end{array}$ & - & - & $\begin{array}{l}\text { UE A stops } \\
\text { TFG1, } \\
\text { generates and } \\
\text { sends } \\
\text { GROUP } \\
\text { CALL } \\
\text { PROBE, and } \\
\text { starts TFG1 } \\
\text { and TFG3 } \\
\end{array}$ & 10.2 .2 .4 .5 .6 \\
\hline 5 & $\begin{array}{c}\text { T0 + } \\
\text { t_A2 + } \\
\text { t_AB2 }\end{array}$ & $\begin{array}{l}\text { UE } \mathbf{B} \text { and } \mathbf{U E} \\
\mathbf{C} \text { receive } \\
\text { GROUP } \\
\text { CALL PROBE }\end{array}$ & - & - & - & $\begin{array}{c}\text { UE } \mathbf{B} \text { and } \\
\text { UE } \mathbf{C} \text { discard } \\
\text { the call } \\
\text { control } \\
\text { message }\end{array}$ & 10.2.2.4.7.1 \\
\hline
\end{tabular}

\subsubsection{Call reject}

In this scenario, UE A decides to establish a group call since there is no existing one. However, UE B rejects the call explicitly, and UE $C$ rejects the call implicitly due to no timely user action. 
Scenario purpose: To observe that a user establishes a new group call, with other users rejecting (in different manners) this call.

Table 28: Call reject

\begin{tabular}{|c|c|c|c|c|c|c|c|}
\hline & Timeline & $\begin{array}{c}\text { Triggering } \\
\text { event }\end{array}$ & $\begin{array}{l}\text { UE A state } \\
\text { transition }\end{array}$ & $\begin{array}{l}\text { UE B state } \\
\text { transition }\end{array}$ & $\begin{array}{l}\text { UE C state } \\
\text { transition }\end{array}$ & $\begin{array}{c}\text { Consequent } \\
\text { behavior }\end{array}$ & $\begin{array}{l}\text { Reference } \\
\text { for details }\end{array}$ \\
\hline 0 & - & - & $\begin{array}{l}\text { S1: start- } \\
\text { stop }\end{array}$ & $\begin{array}{l}\text { S1: start- } \\
\text { stop }\end{array}$ & $\begin{array}{l}\text { S1: start- } \\
\text { stop }\end{array}$ & - & - \\
\hline 1 & T0 & $\begin{array}{l}\text { Indication } \\
\text { from UE A } \\
\text { to initiate a } \\
\text { group call }\end{array}$ & $\begin{array}{c}\text { S2: } \\
\text { waiting for } \\
\text { call } \\
\text { announce } \\
\text { ment }\end{array}$ & - & - & $\begin{array}{c}\text { UE A stores } \\
\text { MCPTT group } \\
\text { ID as MCPTT } \\
\text { group ID of the } \\
\text { call, creates a } \\
\text { Call Type } \\
\text { Control state } \\
\text { machine, } \\
\text { generates and } \\
\text { sends a GROUP } \\
\text { CALL PROBE } \\
\text { message, and } \\
\text { starts TFG1 and } \\
\text { TFG3 } \\
\end{array}$ & 10.2.2.4.2.1 \\
\hline 2 & $\begin{array}{l}\mathrm{T} 0+ \\
\mathrm{t} \_\mathrm{AB} 1\end{array}$ & $\begin{array}{l}\text { UE B and } \\
\text { UE C } \\
\text { receive } \\
\text { GROUP } \\
\text { CALL } \\
\text { PROBE }\end{array}$ & - & - & - & $\begin{array}{l}\text { UE B and UE C } \\
\text { discard the call } \\
\text { control message }\end{array}$ & 10.2 .2 .4 .7 .1 \\
\hline 3 & $\begin{array}{c}\text { T0 + } \\
\text { TFG3 }\end{array}$ & $\begin{array}{l}\text { UE A } \\
\text { TFG3 } \\
\text { expires }\end{array}$ & - & - & - & $\begin{array}{l}\text { UE A generates } \\
\text { and sends } \\
\text { GROUP CALL } \\
\text { PROBE, and } \\
\text { starts TFG3 }\end{array}$ & 10.2.2.4.2.2 \\
\hline 4 & $\begin{array}{c}\text { T0 + } \\
\text { TFG3 + } \\
\text { t_AB2 }\end{array}$ & $\begin{array}{l}\text { UE B and } \\
\text { UE C } \\
\text { receive } \\
\text { GROUP } \\
\text { CALL } \\
\text { PROBE }\end{array}$ & - & - & - & $\begin{array}{l}\text { UE } \mathbf{B} \text { and UE C } \\
\text { discard the call } \\
\text { control message }\end{array}$ & 10.2.2.4.7.1 \\
\hline 5 & $\begin{array}{c}\text { T0 + } \\
\text { n*TFG3 }\end{array}$ & $\begin{array}{l}\text { UE A } \\
\text { TFG3 } \\
\text { expires }\end{array}$ & - & - & - & $\begin{array}{l}\text { UE A generates } \\
\text { and sends } \\
\text { GROUP CALL } \\
\text { PROBE, and } \\
\text { starts TFG3 }\end{array}$ & 10.2.2.4.2.2 \\
\hline
\end{tabular}




\begin{tabular}{|c|c|c|c|c|c|c|c|}
\hline & Timeline & $\begin{array}{c}\text { Triggering } \\
\text { event }\end{array}$ & $\begin{array}{l}\text { UE A state } \\
\text { transition }\end{array}$ & $\begin{array}{l}\text { UE B state } \\
\text { transition }\end{array}$ & $\begin{array}{l}\text { UE C state } \\
\text { transition }\end{array}$ & $\begin{array}{c}\text { Consequent } \\
\text { behavior }\end{array}$ & $\begin{array}{l}\text { Reference } \\
\text { for details }\end{array}$ \\
\hline 6 & $\begin{array}{c}\text { T0 + } \\
\text { n*TFG3 } \\
+\mathrm{t} \_ \text {ABn }\end{array}$ & $\begin{array}{l}\text { UE B and } \\
\text { UE C C } \\
\text { receive } \\
\text { GROUP } \\
\text { CALL } \\
\text { PROBE }\end{array}$ & - & - & - & $\begin{array}{l}\text { UE B and UE C } \\
\text { discard the call } \\
\text { control message }\end{array}$ & 10.2 .2 .4 .7 .1 \\
\hline 7 & $\begin{array}{c}\text { T0 + } \\
\text { TFG1 }\end{array}$ & $\begin{array}{l}\text { UE A } \\
\text { TFG1 } \\
\text { expires }\end{array}$ & $\begin{array}{l}\text { S3: part of } \\
\text { ongoing } \\
\text { call }\end{array}$ & - & - & $\begin{array}{c}\text { UE A stops } \\
\text { TFG3, generates } \\
\text { and stores SDP } \\
\text { body, generates } \\
\text { and stores the call } \\
\text { identifier, selects } \\
\text { and stores refresh } \\
\text { interval, stores its } \\
\text { own MCPTT ID } \\
\text { as originating } \\
\text { MCPTT user ID, } \\
\text { stores current } \\
\text { UTC time as call } \\
\text { start time, } \\
\text { generates and } \\
\text { sends GROUP } \\
\text { CALL } \\
\text { ANNOUNCEME } \\
\text { NT, establishes } \\
\text { media session, } \\
\text { starts Floor } \\
\text { Control as } \\
\text { originating floor } \\
\text { participant, and } \\
\text { starts TFG2 and } \\
\text { TFG6 }\end{array}$ & 10.2 .2 .4 .3 .1 \\
\hline
\end{tabular}




\begin{tabular}{|c|c|c|c|c|c|c|c|}
\hline & Timeline & $\begin{array}{c}\text { Triggering } \\
\text { event }\end{array}$ & $\begin{array}{l}\text { UE A state } \\
\text { transition }\end{array}$ & $\begin{array}{l}\text { UE B state } \\
\text { transition }\end{array}$ & $\begin{array}{l}\text { UE C state } \\
\text { transition }\end{array}$ & $\begin{array}{c}\text { Consequent } \\
\text { behavior }\end{array}$ & $\begin{array}{l}\text { Reference } \\
\text { for details }\end{array}$ \\
\hline 8 & $\begin{array}{c}\text { T0 + } \\
\text { TFG1 + } \\
\text { t_AB(n+ } \\
1)\end{array}$ & $\begin{array}{l}\text { UE B and } \\
\text { UE C } \\
\text { receive } \\
\text { GROUP } \\
\text { CALL } \\
\text { ANNOUN } \\
\text { CEMENT }\end{array}$ & - & $\begin{array}{c}\text { S4: pending } \\
\text { user action } \\
\text { without } \\
\text { confirm } \\
\text { indication } \\
\text { (MCPTT } \\
\text { User } \\
\text { acknowledg } \\
\text { ement } \\
\text { required } \\
\text { upon a } \\
\text { terminating } \\
\text { call request } \\
\text { reception } \\
\text { and GROUP } \\
\text { CALL } \\
\text { ANNNOUN } \\
\text { CEMENT } \\
\text { does not } \\
\text { contain } \\
\text { Confirm } \\
\text { mode } \\
\text { indication } \\
\text { IE) }\end{array}$ & $\begin{array}{c}\text { S4: pending } \\
\text { user action } \\
\text { without } \\
\text { confirm } \\
\text { indication } \\
\text { (MCPTT } \\
\text { User } \\
\text { acknowledg } \\
\text { ement } \\
\text { required } \\
\text { upon a } \\
\text { terminating } \\
\text { call request } \\
\text { reception } \\
\text { and GROUP } \\
\text { CALL } \\
\text { ANNOUNC } \\
\text { EMENT } \\
\text { does not } \\
\text { contain } \\
\text { Confirm } \\
\text { mode } \\
\text { indication } \\
\text { IE) }\end{array}$ & $\begin{array}{c}\text { UE B and UE C } \\
\text { check if MCPTT } \\
\text { group ID IE from } \\
\text { GROUP CALL } \\
\text { ANNOUNCEME } \\
\text { NT does not } \\
\text { match MCPTT } \\
\text { group ID of the } \\
\text { call stored for } \\
\text { other state } \\
\text { machines, store } \\
\text { value of SDP IE, } \\
\text { Call Identifier IE, } \\
\text { Originating } \\
\text { MCPTT user ID } \\
\text { IE, Refresh } \\
\text { Interval IE, } \\
\text { MCPTT Group } \\
\text { ID IE, Call start } \\
\text { time IE of the } \\
\text { GROUP CALL } \\
\text { ANNOUNCEME } \\
\text { NT as } \\
\text { corresponding } \\
\text { values, create } \\
\text { Call Type } \\
\text { Control state } \\
\text { machine, and } \\
\text { start TFG4 }\end{array}$ & 10.2 .2 .4 .3 .3 \\
\hline 9 & $\begin{array}{c}\text { T0 + } \\
\text { t_B1 } \\
(\text { with } \\
t \_B 1> \\
T F G 1+ \\
t \_A B(n+ \\
1)) \\
\end{array}$ & $\begin{array}{c}\text { UE B } \\
\text { rejects } \\
\text { terminating } \\
\text { call }\end{array}$ & - & $\begin{array}{c}\text { S6: } \\
\text { ignoring } \\
\text { incoming } \\
\text { call } \\
\text { announcem } \\
\text { ents }\end{array}$ & - & $\begin{array}{c}\text { UE B stops } \\
\text { TFG4, and starts } \\
\text { TFG5 }\end{array}$ & 10.2 .2 .4 .3 .7 \\
\hline $\begin{array}{l}1 \\
0\end{array}$ & $\begin{array}{c}\text { T0 + } \\
\text { TFG1 + } \\
\text { t_AB(n+ } \\
\text { 1) + } \\
\text { TFG4 }\end{array}$ & $\begin{array}{l}\text { UE C } \\
\text { TFG4 } \\
\text { expires }\end{array}$ & - & - & $\begin{array}{c}\text { S6: } \\
\text { ignoring } \\
\text { incoming } \\
\text { call } \\
\text { announcem } \\
\text { ents }\end{array}$ & $\begin{array}{c}\text { UE C starts } \\
\text { TFG5 }\end{array}$ & 10.2 .2 .4 .3 .8 \\
\hline
\end{tabular}




\subsection{Basic group call - call type control}

The call type control state machine exists when a UE is part of an ongoing group call. The basic call control state machine has a related call type control state machine. The call type control state machine provides additional detail, i.e., call type, about the status of the call taking place: emergency group call, imminent peril group call, and basic group call.

The state transitions that are analyzed in each test scenario of Section 2.3 are based on "Figure 10.2.3.2-1: Call type control state machine" of TS 24.379 v14.2.0 [4]. Call type control test scenarios can be categorized into 5 groups: call type initialization, call type upgrade, call type downgrade, call release, and call merge.

Note: Interaction dependence between basic call control and basic call type control.

\subsubsection{Call type initialization}

\subsubsection{Call type initialization - establish a new basic call}

At the beginning of this scenario, there is no ongoing call established. UE A starts a new basic group call and initializes the call type state machine. It is important to note that this test scenario only depicts the changes happening at the call type state machine. There are other call control messages exchanged in the meanwhile, such as the GROUP CALL ANNOUNCEMENT messages, GROUP CALL PROBE messages, or both.

Scenario purpose: To observe that the "basic group call” type state is entered after creation of a new basic group call.

Table 29: Call type initialization - establish a new basic call

\begin{tabular}{|c|c|c|c|c|c|c|c|}
\hline Timeline & $\begin{array}{c}\text { Triggering } \\
\text { event }\end{array}$ & $\begin{array}{c}\text { UE A state } \\
\text { transition }\end{array}$ & $\begin{array}{c}\text { UE B state } \\
\text { transition }\end{array}$ & $\begin{array}{c}\text { UE C state } \\
\text { transition }\end{array}$ & $\begin{array}{c}\text { Consequent } \\
\text { behavior }\end{array}$ & $\begin{array}{c}\text { Reference } \\
\text { for details }\end{array}$ \\
\hline 0 & - & - & $\begin{array}{c}\text { T0: waiting } \\
\text { for call to } \\
\text { establish }\end{array}$ & $\begin{array}{c}\text { T0: } \\
\text { Waiting } \\
\text { for call to } \\
\text { establish }\end{array}$ & $\begin{array}{c}\text { T0: } \\
\text { Waiting } \\
\text { for call to } \\
\text { establish }\end{array}$ & - & - \\
\hline 1 & T0 & $\begin{array}{c}\text { UE A } \\
\text { initiates } \\
\text { GROUP } \\
\text { CALL } \\
\text { PROBE }\end{array}$ & - & - & - & $\begin{array}{c}\text { Follow 10.2.3.4.2 } \\
\text { for lengthy } \\
\text { detailed behavior } \\
\text { (the stored } \\
\text { current call type } \\
\text { is set to "BASIC } \\
\text { GROUP CALL") }\end{array}$ & $\mathbf{1 0 . 2 . 3 . 4 . 2}$ \\
\hline
\end{tabular}




\begin{tabular}{|c|c|c|c|c|c|c|c|}
\hline & Timeline & $\begin{array}{c}\text { Triggering } \\
\text { event }\end{array}$ & $\begin{array}{c}\text { UE A state } \\
\text { transition }\end{array}$ & $\begin{array}{l}\text { UE B state } \\
\text { transition }\end{array}$ & $\begin{array}{l}\text { UE C state } \\
\text { transition }\end{array}$ & $\begin{array}{c}\begin{array}{c}\text { Consequent } \\
\text { behavior }\end{array} \\
\end{array}$ & $\begin{array}{l}\text { Reference } \\
\text { for details }\end{array}$ \\
\hline 2 & $\begin{array}{c}\text { T0 + } \\
\text { TFG1 }\end{array}$ & $\begin{array}{c}\text { UE A } \\
\text { TFG1 } \\
\text { expires } \\
\text { (hence a } \\
\text { GROUP } \\
\text { CALL } \\
\text { ANNOUN } \\
\text { CEMENT } \\
\text { message } \\
\text { shall be } \\
\text { sent from } \\
\text { the basic } \\
\text { call control } \\
\text { state } \\
\text { machine) }\end{array}$ & $\begin{array}{c}\text { T2: in- } \\
\text { progress } \\
\text { basic group } \\
\text { call }\end{array}$ & - & - & $\begin{array}{l}\text { UE A no action, } \\
\text { except for state } \\
\text { change }\end{array}$ & 10.2.3.4.6 \\
\hline
\end{tabular}

\subsubsection{Call type initialization - establish a new emergency call}

At the beginning of this scenario, there is no ongoing call established. UE A starts a new emergency group call and initializes the call type state machine. It is important to note that this test scenario only depicts the changes happening at the call type state machine. There are other call control messages exchanged in the meanwhile, such as the GROUP CALL ANNOUNCEMENT messages, GROUP CALL PROBE messages, or both.

Scenario purpose: To observe that the "emergency group call” type state is entered after creation of a new emergency group call.

Table 30: Call type initialization - establish a new emergency call

\begin{tabular}{|c|c|c|c|c|c|c|c|}
\hline & Timeline & $\begin{array}{c}\text { Triggering } \\
\text { event }\end{array}$ & $\begin{array}{c}\text { UE A state } \\
\text { transition }\end{array}$ & $\begin{array}{c}\text { UE B state } \\
\text { transition }\end{array}$ & $\begin{array}{c}\text { UE C state } \\
\text { transition }\end{array}$ & $\begin{array}{c}\text { Consequent } \\
\text { behavior }\end{array}$ & $\begin{array}{c}\text { Reference } \\
\text { for details }\end{array}$ \\
\hline 0 & - & - & $\begin{array}{c}\text { T0: waiting } \\
\text { for call to } \\
\text { establish }\end{array}$ & $\begin{array}{c}\text { T0: } \\
\text { Waiting } \\
\text { for call to } \\
\text { establish }\end{array}$ & $\begin{array}{c}\text { T0: } \\
\text { Waiting } \\
\text { for call to } \\
\text { establish }\end{array}$ & - & - \\
\hline 1 & & & & & $\begin{array}{c}\text { Follow } 10.2 .3 .4 .2 \\
\text { for lengthy } \\
\text { detailed behavior } \\
\text { (the stored } \\
\text { current call type } \\
\text { is set to } \\
\text { T0MERGENCY } \\
\text { GROUP CALL") }\end{array}$ & $\mathbf{1 0 . 2 . 3 . 4 . 2}$ \\
\hline & $\begin{array}{c}\text { UE A } \\
\text { initiates } \\
\text { GROUP } \\
\text { CALL } \\
\text { PROBE }\end{array}$ & - & - & - & & & \\
\hline
\end{tabular}




\begin{tabular}{|c|c|c|c|c|c|c|c|}
\hline & Timeline & $\begin{array}{c}\text { Triggering } \\
\text { event }\end{array}$ & $\begin{array}{l}\text { UE A state } \\
\text { transition }\end{array}$ & $\begin{array}{l}\text { UE B state } \\
\text { transition }\end{array}$ & $\begin{array}{l}\text { UE C state } \\
\text { transition }\end{array}$ & $\begin{array}{c}\begin{array}{c}\text { Consequent } \\
\text { behavior }\end{array} \\
\end{array}$ & $\begin{array}{l}\text { Reference } \\
\text { for details }\end{array}$ \\
\hline 2 & $\begin{array}{c}\text { T0 + } \\
\text { TFG1 }\end{array}$ & $\begin{array}{c}\text { UE A } \\
\text { TFG1 } \\
\text { expires } \\
\text { (hence a } \\
\text { GROUP } \\
\text { CALL } \\
\text { ANNOUN } \\
\text { CEMENT } \\
\text { message } \\
\text { shall be } \\
\text { sent from } \\
\text { the basic } \\
\text { call control } \\
\text { state } \\
\text { machine) }\end{array}$ & $\begin{array}{l}\text { T1: in- } \\
\text { progress } \\
\text { emergency } \\
\text { group call }\end{array}$ & - & - & $\begin{array}{c}\text { UE A starts } \\
\text { TFG13 }\end{array}$ & 10.2 .3 .4 .6 \\
\hline
\end{tabular}

\subsubsection{Call type initialization - establish a new imminent peril call}

At the beginning of this scenario, there is no ongoing call established. UE A starts a new imminent peril group call and initializes the call type state machine. It is important to note that this test scenario only depicts the changes happening at the call type state machine. There are other call control messages exchanged in the meanwhile, such as the GROUP CALL ANNOUNCEMENT messages, GROUP CALL PROBE messages, or both.

Scenario purpose: To observe that the "imminent peril group call” type state is entered after creation of a new imminent peril group call.

Table 31: Call type initialization - establish a new imminent peril call

\begin{tabular}{|c|c|c|c|c|c|c|c|}
\hline & Timeline & $\begin{array}{c}\text { Triggering } \\
\text { event }\end{array}$ & $\begin{array}{c}\text { UE A state } \\
\text { transition }\end{array}$ & $\begin{array}{l}\text { UE B state } \\
\text { transition }\end{array}$ & $\begin{array}{l}\text { UE C state } \\
\text { transition }\end{array}$ & $\begin{array}{c}\text { Consequent } \\
\text { behavior }\end{array}$ & $\begin{array}{l}\text { Reference } \\
\text { for details } \\
\end{array}$ \\
\hline 0 & - & - & $\begin{array}{l}\text { T0: waiting } \\
\text { for call to } \\
\text { establish }\end{array}$ & $\begin{array}{c}\text { T0: } \\
\text { Waiting } \\
\text { for call to } \\
\text { establish } \\
\end{array}$ & $\begin{array}{c}\text { T0: } \\
\text { Waiting } \\
\text { for call to } \\
\text { establish } \\
\end{array}$ & 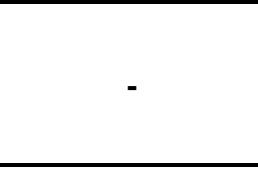 & 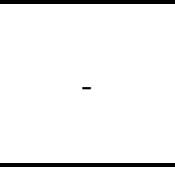 \\
\hline 1 & T0 & $\begin{array}{c}\text { UE A } \\
\text { initiates } \\
\text { GROUP } \\
\text { CALL } \\
\text { PROBE }\end{array}$ & - & - & - & $\begin{array}{l}\text { Follow 10.2.3.4.2 } \\
\text { for lengthy } \\
\text { detailed behavior } \\
\text { (the stored } \\
\text { current call type } \\
\text { is set to } \\
\text { "IMMINENT } \\
\text { PERIL GROUP } \\
\text { CALL") }\end{array}$ & 10.2.3.4.2 \\
\hline
\end{tabular}




\begin{tabular}{|c|c|c|c|c|c|c|c|}
\hline & Timeline & $\begin{array}{c}\text { Triggering } \\
\text { event }\end{array}$ & $\begin{array}{l}\text { UE A state } \\
\text { transition }\end{array}$ & $\begin{array}{l}\text { UE B state } \\
\text { transition }\end{array}$ & $\begin{array}{l}\text { UE C state } \\
\text { transition }\end{array}$ & $\begin{array}{c}\text { Consequent } \\
\text { behavior }\end{array}$ & $\begin{array}{l}\text { Reference } \\
\text { for details }\end{array}$ \\
\hline 2 & $\begin{array}{c}\text { T0 + } \\
\text { TFG1 }\end{array}$ & $\begin{array}{c}\text { UE A } \\
\text { TFG1 } \\
\text { expires } \\
\text { (hence a } \\
\text { GROUP } \\
\text { CALL } \\
\text { ANNOUN } \\
\text { CEMENT } \\
\text { message } \\
\text { shall be } \\
\text { sent from } \\
\text { the basic } \\
\text { call control } \\
\text { state } \\
\text { machine) }\end{array}$ & $\begin{array}{c}\text { T3: in- } \\
\text { progress } \\
\text { imminent } \\
\text { peril group } \\
\text { call }\end{array}$ & - & - & $\begin{array}{c}\text { UE A starts } \\
\text { TFG14 }\end{array}$ & 10.2 .3 .4 .6 \\
\hline
\end{tabular}

\subsubsection{Call type initialization - join an emergency call after call probe}

In this scenario, there is an ongoing emergency group call. UE A sends a call probe and joins this emergency group call.

Scenario purpose: To observe that the emergency call is joined after receiving an emergency-based GROUP CALL ANNOUNCEMENT message.

Table 32: Call type initialization - join an emergency call after call probe

\begin{tabular}{|c|c|c|c|c|c|c|c|}
\hline & Timeline & $\begin{array}{c}\text { Triggering } \\
\text { event }\end{array}$ & $\begin{array}{l}\text { UE A state } \\
\text { transition }\end{array}$ & $\begin{array}{l}\text { UE B state } \\
\text { transition }\end{array}$ & $\begin{array}{l}\text { UE C state } \\
\text { transition }\end{array}$ & $\begin{array}{c}\text { Consequent } \\
\text { behavior }\end{array}$ & $\begin{array}{l}\text { Reference } \\
\text { for details }\end{array}$ \\
\hline 0 & - & - & $\begin{array}{l}\text { T0: waiting } \\
\text { for call to } \\
\text { establish }\end{array}$ & $\begin{array}{c}\text { T1: in- } \\
\text { progress } \\
\text { emergency } \\
\text { group call }\end{array}$ & $\begin{array}{c}\text { T1: in- } \\
\text { progress } \\
\text { emergency } \\
\text { group call }\end{array}$ & - & - \\
\hline 1 & T0 & $\begin{array}{c}\text { UE A } \\
\text { initiates } \\
\text { GROUP } \\
\text { CALL } \\
\text { PROBE } \\
\end{array}$ & - & - & - & $\begin{array}{c}\text { Follow } \\
\text { 10.2.3.4.2 for } \\
\text { lengthy detailed } \\
\text { behavior }\end{array}$ & 10.2 .3 .4 .2 \\
\hline 2 & $\begin{array}{l}\text { T0 + } \\
\text { t_B1 }\end{array}$ & $\begin{array}{l}\text { UE B } \\
\text { TFG2 } \\
\text { expires }\end{array}$ & & & & $\begin{array}{c}\text { UE B sends a } \\
\text { GROUP CALL } \\
\text { ANNOUNCEM } \\
\text { ENT }\end{array}$ & $\begin{array}{r}\text { NOTE: } \\
\text { happens } \\
\text { outside } \\
\text { call type } \\
\text { control }\end{array}$ \\
\hline
\end{tabular}




\begin{tabular}{|c|c|c|c|c|c|c|c|}
\hline & Timeline & $\begin{array}{c}\text { Triggering } \\
\text { event }\end{array}$ & $\begin{array}{l}\text { UE A state } \\
\text { transition }\end{array}$ & $\begin{array}{l}\text { UE B state } \\
\text { transition }\end{array}$ & $\begin{array}{l}\text { UE C state } \\
\text { transition }\end{array}$ & $\begin{array}{c}\text { Consequent } \\
\text { behavior }\end{array}$ & $\begin{array}{l}\text { Reference } \\
\text { for details }\end{array}$ \\
\hline 3 & $\begin{array}{l}\text { T0 + } \\
\text { t_B1 + } \\
\text { t_BA1 }\end{array}$ & $\begin{array}{c}\text { UE A } \\
\text { receives } \\
\text { GROUP } \\
\text { CALL } \\
\text { ANNOUN } \\
\text { CEMENT } \\
\text { from UE B }\end{array}$ & $\begin{array}{l}\text { T1: in- } \\
\text { progress } \\
\text { emergency } \\
\text { group call }\end{array}$ & - & - & $\begin{array}{c}\text { UE A sets } \\
\text { current call type } \\
\text { to } \\
\text { "EMERGENC } \\
\text { Y GROUP } \\
\text { CALL", sets } \\
\text { Proximity } \\
\text { Based Services } \\
\text { (ProSe) per- } \\
\text { packet priority } \\
\text { to value of } \\
\text { MCPTT off- } \\
\text { network } \\
\text { emergency } \\
\text { group call, sets } \\
\text { stored last call } \\
\text { type change } \\
\text { time and last } \\
\text { user to change } \\
\text { call type to } \\
\text { corresponding } \\
\text { values of } \\
\text { GROUP CALL } \\
\text { ANNOUNCEM } \\
\text { ENT, and starts } \\
\text { TFG13 }\end{array}$ & 10.2.3.4.3 \\
\hline
\end{tabular}

\subsubsection{Call type initialization - join an imminent peril call after call probe}

In this scenario, there is an ongoing imminent peril group call. UE A sends a GROUP CALL PROBE and joins this imminent peril group call.

Scenario purpose: To observe that the imminent peril call is joined after receiving an imminent peril-based GROUP CALL ANNOUNCEMENT message.

Table 33: Call type initialization - join an imminent peril call after GROUP CALL PROBE

\begin{tabular}{|c|c|c|c|c|c|c|c|}
\hline & Timeline & $\begin{array}{c}\text { Triggering } \\
\text { event }\end{array}$ & $\begin{array}{c}\text { UE A state } \\
\text { transition }\end{array}$ & $\begin{array}{c}\text { UE B state } \\
\text { transition }\end{array}$ & $\begin{array}{c}\text { UE C state } \\
\text { transition }\end{array}$ & $\begin{array}{c}\text { Consequent } \\
\text { behavior }\end{array}$ & $\begin{array}{c}\text { Reference } \\
\text { for details }\end{array}$ \\
\hline 0 & & & $\begin{array}{c}\text { T0: } \\
\text { waiting for } \\
\text { call to } \\
\text { establish }\end{array}$ & $\begin{array}{c}\text { T3: in- } \\
\text { progress } \\
\text { imminent } \\
\text { peril group } \\
\text { call }\end{array}$ & $\begin{array}{c}\text { T3: in- } \\
\text { progress } \\
\text { imminent } \\
\text { peril group } \\
\text { call }\end{array}$ & & \\
-- & - & & & - & - \\
\hline 1 & T0 & $\begin{array}{c}\text { UE A } \\
\text { initiates } \\
\text { GROUP }\end{array}$ & - & - & - & $\begin{array}{l}\text { Follow } \\
\text { leng.2.3.4.2 for } \\
\text { lengthy detailed } \\
\text { behavior }\end{array}$ & $\mathbf{1 0 . 2 . 3 . 4 . 2}$ \\
\hline
\end{tabular}




\begin{tabular}{|c|c|c|c|c|c|c|c|}
\hline & Timeline & $\begin{array}{c}\text { Triggering } \\
\text { event }\end{array}$ & $\begin{array}{l}\text { UE A state } \\
\text { transition }\end{array}$ & $\begin{array}{l}\text { UE B state } \\
\text { transition }\end{array}$ & $\begin{array}{l}\text { UE C state } \\
\text { transition }\end{array}$ & $\begin{array}{c}\text { Consequent } \\
\text { behavior }\end{array}$ & $\begin{array}{l}\text { Reference } \\
\text { for details }\end{array}$ \\
\hline 2 & $\begin{array}{l}\text { T0 + } \\
\text { t_B1 }\end{array}$ & $\begin{array}{l}\text { UE B } \\
\text { TFG2 } \\
\text { expires }\end{array}$ & & & & $\begin{array}{l}\text { UE B sends a } \\
\text { GROUP CALL } \\
\text { ANNOUNCEM } \\
\text { ENT }\end{array}$ & $\begin{array}{r}\text { NOTE: } \\
\text { happens } \\
\text { outside } \\
\text { call type } \\
\text { control }\end{array}$ \\
\hline 3 & $\begin{array}{c}\text { T0 + } \\
\text { t_B1 + } \\
\text { t_BA1 }\end{array}$ & $\begin{array}{c}\text { UE A } \\
\text { receives } \\
\text { GROUP } \\
\text { CALL } \\
\text { ANNNOU } \\
\text { NCEMEN } \\
\text { T from UE } \\
\text { B }\end{array}$ & $\begin{array}{c}\text { T3: in- } \\
\text { progress } \\
\text { imminent } \\
\text { peril } \\
\text { group call }\end{array}$ & - & - & $\begin{array}{l}\text { UE A checks if } \\
\text { current call type } \\
\text { is not } \\
\text { "EMERGENC } \\
\text { Y GROUP } \\
\text { CALL", sets } \\
\text { current call type } \\
\text { to "IMMINENT } \\
\text { PERIL GROUP } \\
\text { CALL", sets } \\
\text { ProSe per- } \\
\text { packet priority } \\
\text { to value of } \\
\text { MCPTT off- } \\
\text { network } \\
\text { imminent peril } \\
\text { group call, sets } \\
\text { stored last call } \\
\text { type change } \\
\text { time and last } \\
\text { user to change } \\
\text { call type to } \\
\text { corresponding } \\
\text { values of } \\
\text { GROUP CALL } \\
\text { ANNOUNCEM } \\
\text { ENT, and starts } \\
\text { TFG14 }\end{array}$ & 10.2 .3 .4 .3 \\
\hline
\end{tabular}

\subsubsection{Call type initialization - join a basic group call after group call probe}

In this scenario, there is an ongoing basic group call. UE A sends a GROUP CALL PROBE message and joins this basic group call.

Scenario purpose: To observe that the basic group call is joined after receiving a basic group call-based GROUP CALL ANNOUNCEMENT message.

Table 34: Call type initialization - join a basic group call after group call probe

\begin{tabular}{|c|c|c|c|c|c|c|c|}
\hline & Timeline & $\begin{array}{c}\text { Triggering } \\
\text { event }\end{array}$ & $\begin{array}{c}\text { UE A state } \\
\text { transition }\end{array}$ & $\begin{array}{c}\text { UE B state } \\
\text { transition }\end{array}$ & $\begin{array}{c}\text { UE C state } \\
\text { transition }\end{array}$ & $\begin{array}{c}\text { Consequent } \\
\text { behavior }\end{array}$ & $\begin{array}{c}\text { Reference } \\
\text { for details }\end{array}$ \\
\hline 0 & - & - & $\begin{array}{c}\text { T0: waiting } \\
\text { for call to } \\
\text { establish }\end{array}$ & $\begin{array}{c}\text { T2: in- } \\
\text { progress } \\
\text { basic group } \\
\text { call }\end{array}$ & $\begin{array}{c}\text { T2: in- } \\
\text { progress } \\
\text { basic group } \\
\text { call }\end{array}$ & - & - \\
\hline
\end{tabular}




\begin{tabular}{|c|c|c|c|c|c|c|c|}
\hline & Timeline & $\begin{array}{c}\text { Triggering } \\
\text { event }\end{array}$ & $\begin{array}{l}\text { UE A state } \\
\text { transition }\end{array}$ & $\begin{array}{l}\text { UE B state } \\
\text { transition }\end{array}$ & $\begin{array}{l}\text { UE C state } \\
\text { transition }\end{array}$ & $\begin{array}{c}\text { Consequent } \\
\text { behavior }\end{array}$ & $\begin{array}{l}\text { Reference } \\
\text { for details }\end{array}$ \\
\hline 1 & T0 & $\begin{array}{c}\text { UE A } \\
\text { initiates } \\
\text { GROUP } \\
\text { CALL } \\
\text { PROBE }\end{array}$ & - & - & - & $\begin{array}{c}\text { Follow } \\
\text { 10.2.3.4.2 for } \\
\text { lengthy } \\
\text { detailed } \\
\text { behavior }\end{array}$ & 10.2.3.4.2 \\
\hline 2 & T0 + t_B1 & $\begin{array}{c}\text { Either UE } \\
\text { B or UE C } \\
\text { TFG2 } \\
\text { expires }\end{array}$ & & & & $\begin{array}{c}\text { UE B or UE C } \\
\text { sends a } \\
\text { GROUP } \\
\text { CALL } \\
\text { ANNOUNCE } \\
\text { MENT } \\
\end{array}$ & $\begin{array}{r}\text { NOTE: } \\
\text { happens } \\
\text { outside } \\
\text { call type } \\
\text { control }\end{array}$ \\
\hline 3 & $\begin{array}{c}\text { T0 + t_B1 } \\
+\mathrm{t} \_\mathrm{BA} 1\end{array}$ & $\begin{array}{c}\text { UE A } \\
\text { receives } \\
\text { GROUP } \\
\text { CALL } \\
\text { ANNOUN } \\
\text { CEMENT }\end{array}$ & $\begin{array}{l}\text { T2: in- } \\
\text { progress } \\
\text { basic group } \\
\text { call }\end{array}$ & - & - & $\begin{array}{l}\text { UE A checks } \\
\text { if current call } \\
\text { type is not } \\
\text { "EMERGENC } \\
\text { Y GROUP } \\
\text { CALL", sets } \\
\text { current call } \\
\text { type to } \\
\text { "BASIC } \\
\text { GROUP } \\
\text { CALL", and } \\
\text { sets stored last } \\
\text { call type } \\
\text { change time } \\
\text { and last user to } \\
\text { change call } \\
\text { type to } \\
\text { corresponding } \\
\text { values of } \\
\text { GROUP } \\
\text { CALL } \\
\text { ANNOUNCE } \\
\text { MENT }\end{array}$ & 10.2.3.4.3 \\
\hline
\end{tabular}

\subsubsection{Call type initialization - join a basic group call with user acknowledgement required}

In this scenario, a basic group call is ongoing. UE A receives a group call announcement message of that call which requires user acknowledgment. The MCPTT user of UE A accepts the call.

Scenario purpose: To observe that the basic group call state is entered after joining an already established basic group call (with acknowledgement required). 
Table 35: Call type initialization - join a basic group call with user acknowledgement required

\begin{tabular}{|c|c|c|c|c|c|c|c|}
\hline & Timeline & $\begin{array}{c}\text { Triggering } \\
\text { event }\end{array}$ & $\begin{array}{l}\text { UE A state } \\
\text { transition }\end{array}$ & $\begin{array}{l}\text { UE B state } \\
\text { transition }\end{array}$ & $\begin{array}{l}\text { UE C state } \\
\text { transition }\end{array}$ & $\begin{array}{c}\text { Consequent } \\
\text { behavior }\end{array}$ & $\begin{array}{c}\text { Referenc } \\
\text { e for } \\
\text { details } \\
\end{array}$ \\
\hline 0 & - & - & $\begin{array}{c}\text { T0: } \\
\text { waiting for } \\
\text { call to } \\
\text { establish }\end{array}$ & $\begin{array}{c}\text { T2: in- } \\
\text { progress } \\
\text { basic } \\
\text { group call }\end{array}$ & $\begin{array}{c}\text { T2: in- } \\
\text { progress } \\
\text { basic } \\
\text { group call }\end{array}$ & - & - \\
\hline 1 & T0 & $\begin{array}{l}\text { UE B } \\
\text { TFG2 } \\
\text { expires }\end{array}$ & - & - & - & $\begin{array}{c}\text { UE B sends a } \\
\text { GROUP CALL } \\
\text { ANNOUNCEMENT } \\
\text { (with Call type IE set } \\
\text { to "BASIC GROUP } \\
\text { CALL") } \\
\end{array}$ & $\begin{array}{r}\text { NOTE: } \\
\text { happens } \\
\text { outside } \\
\text { call type } \\
\text { control }\end{array}$ \\
\hline 2 & $\begin{array}{l}\text { T0 + } \\
\text { t_BA1 }\end{array}$ & $\begin{array}{c}\text { UE A } \\
\text { receives } \\
\text { GROUP } \\
\text { CALL } \\
\text { ANNOUN } \\
\text { CEMENT } \\
\text { (with } \\
\text { MCPTT } \\
\text { user } \\
\text { acknowled } \\
\text { gement } \\
\text { required) }\end{array}$ & - & - & - & $\begin{array}{l}\text { Follow 10.2.3.4.4. for } \\
\text { lengthy detailed } \\
\text { behavior } \\
\text { UE A stores current } \\
\text { call type as "BASIC } \\
\text { GROUP CALL" }\end{array}$ & 10.2 .3 .4 .4 \\
\hline 3 & $\begin{array}{l}\text { T0 + } \\
\text { t_A1 } \\
\text { (with } \\
\text { t_A1 > } \\
\text { t_BA1) }\end{array}$ & $\begin{array}{l}\text { UE A } \\
\text { accepts the } \\
\text { call }\end{array}$ & $\begin{array}{l}\text { T2: in- } \\
\text { progress } \\
\text { basic } \\
\text { group call }\end{array}$ & - & - & $\begin{array}{l}\text { UE A no action, } \\
\text { except for state } \\
\text { change }\end{array}$ & 10.2.3.4.6 \\
\hline
\end{tabular}

\subsubsection{Call type initialization - join an imminent peril group call with user acknowledgement required}

In this scenario, an imminent peril group call is ongoing. UE A receives a group call announcement message of that call which requires user acknowledgment. The MCPTT user of UE A accepts the call.

Scenario purpose: To observe that the imminent peril call state is entered after joining an already established imminent peril group call (with acknowledgement required). 
Table 36: Call type initialization - join an imminent peril group call with user acknowledgement required

\begin{tabular}{|c|c|c|c|c|c|c|c|}
\hline & Timeline & $\begin{array}{c}\text { Triggering } \\
\text { event }\end{array}$ & $\begin{array}{l}\text { UE A state } \\
\text { transition }\end{array}$ & $\begin{array}{l}\text { UE B state } \\
\text { transition }\end{array}$ & $\begin{array}{l}\text { UE C state } \\
\text { transition }\end{array}$ & $\begin{array}{c}\text { Consequent } \\
\text { behavior }\end{array}$ & $\begin{array}{c}\text { Referenc } \\
\text { e for } \\
\text { details }\end{array}$ \\
\hline 0 & - & - & $\begin{array}{c}\text { T0: } \\
\text { waiting for } \\
\text { call to } \\
\text { establish }\end{array}$ & $\begin{array}{c}\text { T3: in- } \\
\text { progress } \\
\text { imminent } \\
\text { peril } \\
\text { group call }\end{array}$ & $\begin{array}{c}\text { T3: in- } \\
\text { progress } \\
\text { imminent } \\
\text { peril } \\
\text { group call }\end{array}$ & - & - \\
\hline 1 & T0 & $\begin{array}{c}\text { UE B } \\
\text { TFG2 } \\
\text { expires }\end{array}$ & - & - & - & $\begin{array}{c}\text { UE B sends a } \\
\text { GROUP CALL } \\
\text { ANNOUNCEMENT } \\
\text { (with Call type IE set } \\
\text { to "IMMINENT } \\
\text { PERIL GROUP } \\
\text { CALL") }\end{array}$ & $\begin{array}{r}\text { NOTE: } \\
\text { happens } \\
\text { outside } \\
\text { call type } \\
\text { control }\end{array}$ \\
\hline 2 & $\begin{array}{c}\text { T0 + } \\
\text { t_BA1 }\end{array}$ & $\begin{array}{c}\text { UE A } \\
\text { receives } \\
\text { GROUP } \\
\text { CALL } \\
\text { ANNOUN } \\
\text { CEMENT } \\
\text { (with } \\
\text { MCPTT } \\
\text { user } \\
\text { acknowled } \\
\text { gement } \\
\text { required) } \\
\end{array}$ & - & - & - & $\begin{array}{l}\text { Follow 10.2.3.4.4. for } \\
\text { lengthy detailed } \\
\text { behavior } \\
\text { UE A stores current } \\
\text { call type as } \\
\text { "IMMINENT PERIL } \\
\text { GROUP CALL" }\end{array}$ & 10.2.3.4.4 \\
\hline 3 & $\begin{array}{l}\mathrm{T} 0+ \\
\mathrm{t} \_A 1 \\
(w i t h \\
t \_A 1> \\
\left.t \_B A 1\right)\end{array}$ & $\begin{array}{l}\text { UE A } \\
\text { accepts the } \\
\text { call }\end{array}$ & $\begin{array}{c}\text { T3: in- } \\
\text { progress } \\
\text { imminent } \\
\text { peril } \\
\text { group call }\end{array}$ & - & - & UE A starts TFG14 & 10.2.3.4.6 \\
\hline
\end{tabular}

\subsubsection{Call type initialization - join an emergency group call with user acknowledgement required}

In this scenario, an emergency group call is ongoing. UE A receives a group call announcement message of that call which requires user acknowledgment. The MCPTT user of UE A accepts the call.

Scenario purpose: To observe that the emergency group call state is entered after joining an already established emergency group call. 
Table 37: Call type initialization - join an emergency group call with user acknowledgement required

\begin{tabular}{|c|c|c|c|c|c|c|c|}
\hline & Timeline & $\begin{array}{c}\text { Triggering } \\
\text { event }\end{array}$ & $\begin{array}{l}\text { UE A state } \\
\text { transition }\end{array}$ & $\begin{array}{l}\text { UE B state } \\
\text { transition }\end{array}$ & $\begin{array}{l}\text { UE C state } \\
\text { transition }\end{array}$ & $\begin{array}{c}\text { Consequent } \\
\text { behavior }\end{array}$ & $\begin{array}{c}\text { Referenc } \\
\text { e for } \\
\text { details }\end{array}$ \\
\hline 0 & - & - & $\begin{array}{c}\text { T0: } \\
\text { waiting for } \\
\text { call to } \\
\text { establish }\end{array}$ & $\begin{array}{c}\text { T1: in- } \\
\text { progress } \\
\text { emergency } \\
\text { group call }\end{array}$ & $\begin{array}{c}\text { T1: in- } \\
\text { progress } \\
\text { emergency } \\
\text { group call }\end{array}$ & - & - \\
\hline 1 & T0 & $\begin{array}{l}\text { UE B } \\
\text { TFG2 } \\
\text { expires }\end{array}$ & - & - & - & $\begin{array}{c}\text { UE B sends a } \\
\text { GROUP CALL } \\
\text { ANNOUNCEMENT } \\
\text { (with Call type IE set } \\
\text { to "EMERGENCY } \\
\text { GROUP CALL") }\end{array}$ & $\begin{array}{r}\text { NOTE: } \\
\text { happens } \\
\text { outside } \\
\text { call type } \\
\text { control }\end{array}$ \\
\hline 2 & $\begin{array}{l}\text { T0 + } \\
\text { t_BA1 }\end{array}$ & $\begin{array}{c}\text { UE A } \\
\text { receives } \\
\text { GROUP } \\
\text { CALL } \\
\text { ANNOUN } \\
\text { CEMENT } \\
\text { (with } \\
\text { MCPTT } \\
\text { user } \\
\text { acknowled } \\
\text { gement } \\
\text { required) }\end{array}$ & - & - & - & $\begin{array}{l}\text { Follow 10.2.3.4.4. for } \\
\text { lengthy detailed } \\
\text { behavior } \\
\text { UE A stores current } \\
\text { call type as } \\
\text { "EMERGENCY } \\
\text { GROUP CALL" }\end{array}$ & 10.2.3.4.4 \\
\hline$\overline{3}$ & $\begin{array}{l}\mathrm{T} 0+ \\
\mathrm{t} \_A 1 \\
(w i t h \\
t \_A 1> \\
\left.t \_B A 1\right)\end{array}$ & $\begin{array}{l}\text { UE A } \\
\text { accepts the } \\
\text { call }\end{array}$ & $\begin{array}{l}\text { T1: in- } \\
\text { progress } \\
\text { emergency } \\
\text { group call }\end{array}$ & - & - & UE A starts TFG13 & 10.2.3.4.6 \\
\hline
\end{tabular}

\subsubsection{Call type initialization - join an emergency group call without user acknowledgement required}

In this scenario, an emergency group call is ongoing. UE A receives a group call announcement that does not require user acknowledgment and joins the call. This scenario can be generalized to other scenarios in which the ongoing group call is basic or imminent peril.

Scenario purpose: To observe that the emergency call state is entered after joining an already established emergency group call (without acknowledgement required), based on the call type setting in the GROUP CALL ANNOUNCEMENT message. 
Table 38: Call type initialization - join an emergency group call without user acknowledgement required

\begin{tabular}{|c|c|c|c|c|c|c|c|}
\hline & Timeline & $\begin{array}{c}\text { Triggering } \\
\text { event }\end{array}$ & $\begin{array}{l}\text { UE A state } \\
\text { transition }\end{array}$ & $\begin{array}{l}\text { UE B state } \\
\text { transition }\end{array}$ & $\begin{array}{l}\text { UE C state } \\
\text { transition }\end{array}$ & $\begin{array}{c}\text { Consequent } \\
\text { behavior }\end{array}$ & $\begin{array}{c}\text { Referenc } \\
\text { e for } \\
\text { details }\end{array}$ \\
\hline 0 & - & - & $\begin{array}{l}\text { T0: waiting } \\
\text { for call to } \\
\text { establish }\end{array}$ & $\begin{array}{l}\text { T1: in- } \\
\text { progress } \\
\text { emergency } \\
\text { group call }\end{array}$ & $\begin{array}{l}\text { T1: in- } \\
\text { progress } \\
\text { emergency } \\
\text { group }\end{array}$ & - & - \\
\hline 1 & T0 & $\begin{array}{c}\text { UE B } \\
\text { TFG2 } \\
\text { expires }\end{array}$ & - & - & - & $\begin{array}{l}\text { UE B sends a } \\
\text { GROUP CALL } \\
\text { ANNOUNCEM } \\
\text { ENT (with Call } \\
\text { type IE set to } \\
\text { "EMERGENCY } \\
\text { GROUP CALL") }\end{array}$ & $\begin{array}{r}\text { NOTE: } \\
\text { happens } \\
\text { outside } \\
\text { call type } \\
\text { control }\end{array}$ \\
\hline 2 & $\begin{array}{c}\text { T0 + } \\
\text { t_BA1 }\end{array}$ & $\begin{array}{c}\text { UE A } \\
\text { receives } \\
\text { GROUP } \\
\text { CALL } \\
\text { ANNOUN } \\
\text { CEMENT } \\
\text { (without } \\
\text { MCPTT } \\
\text { user } \\
\text { acknowled } \\
\text { gement } \\
\text { required) } \\
\end{array}$ & $\begin{array}{l}\text { T1: in- } \\
\text { progress } \\
\text { emergency } \\
\text { group call }\end{array}$ & - & - & $\begin{array}{c}\text { Follow } \\
\text { 10.2.3.4.5 for } \\
\text { lengthy detailed } \\
\text { behavior } \\
\text { UE A stores } \\
\text { current call type } \\
\text { as set to } \\
\text { "EMERGENCY } \\
\text { GROUP CALL" }\end{array}$ & 10.2 .3 .4 .5 \\
\hline
\end{tabular}

\subsubsection{Call type upgrade}

\subsubsection{Call upgrade from basic to imminent peril group call}

In this scenario, a basic group call is ongoing. A user decides to upgrade the call to an imminent peril group call, so other users' call types are upgraded accordingly.

Scenario purpose: To observe that a basic group call can be upgraded to an imminent peril group call.

Table 39: Call upgrade from basic to imminent peril group call

\begin{tabular}{|c|c|c|c|c|c|c|c|}
\hline Timeline & $\begin{array}{c}\text { Triggering } \\
\text { event }\end{array}$ & $\begin{array}{c}\text { UE A state } \\
\text { transition }\end{array}$ & $\begin{array}{c}\text { UE B state } \\
\text { transition }\end{array}$ & $\begin{array}{c}\text { UE C state } \\
\text { transition }\end{array}$ & $\begin{array}{c}\text { Consequent } \\
\text { behavior }\end{array}$ & $\begin{array}{c}\text { Reference } \\
\text { for details }\end{array}$ \\
\hline 0 & - & - & $\begin{array}{c}\text { T2: in- } \\
\text { progress } \\
\text { basic group } \\
\text { call }\end{array}$ & $\begin{array}{c}\text { T2: in- } \\
\text { progress } \\
\text { basic } \\
\text { group call }\end{array}$ & $\begin{array}{c}\text { T2: in- } \\
\text { progress } \\
\text { basic } \\
\text { group call }\end{array}$ & - & - \\
\hline
\end{tabular}




\begin{tabular}{|c|c|c|c|c|c|c|c|}
\hline & Timeline & $\begin{array}{c}\text { Triggering } \\
\text { event }\end{array}$ & $\begin{array}{l}\text { UE A state } \\
\text { transition }\end{array}$ & $\begin{array}{l}\text { UE B state } \\
\text { transition }\end{array}$ & $\begin{array}{l}\text { UE C state } \\
\text { transition }\end{array}$ & $\begin{array}{c}\text { Consequent } \\
\text { behavior }\end{array}$ & $\begin{array}{l}\text { Reference } \\
\text { for details }\end{array}$ \\
\hline 1 & T0 & $\begin{array}{l}\text { UE A } \\
\text { requests to } \\
\text { upgrade the } \\
\text { call to } \\
\text { "IMMINEN } \\
\text { T PERIL } \\
\text { GROUP } \\
\text { CALL" }\end{array}$ & $\begin{array}{c}\text { T3: in- } \\
\text { progress } \\
\text { imminent } \\
\text { peril group } \\
\text { call }\end{array}$ & - & - & $\begin{array}{c}\text { UE A sets } \\
\text { current call type } \\
\text { to "IMMINENT } \\
\text { PERIL GROUP } \\
\text { CALL" and } \\
\text { accordingly sets } \\
\text { ProSe per-packet } \\
\text { priority, starts } \\
\text { TFG14, stores } \\
\text { UTC time as last } \\
\text { call type change } \\
\text { time of the call as } \\
\text { well as own } \\
\text { MCPTT user ID } \\
\text { as last user to } \\
\text { change call type } \\
\text { of the call, } \\
\text { generates and } \\
\text { sends GROUP } \\
\text { CALL } \\
\text { ANNOUNCEME } \\
\text { NT } \\
\end{array}$ & 10.2.3.4.7.1 \\
\hline 2 & $\begin{array}{c}\text { T0 + } \\
\text { t_AB1 }\end{array}$ & $\begin{array}{l}\text { UE B and } \\
\text { UE C } \\
\text { receive } \\
\text { GROUP } \\
\text { CALL } \\
\text { ANNOUN } \\
\text { CEMENT }\end{array}$ & - & $\begin{array}{c}\text { T3: } \\
\text { imminent } \\
\text { peril } \\
\text { group call }\end{array}$ & $\begin{array}{c}\text { T3: } \\
\text { imminent } \\
\text { peril } \\
\text { group call }\end{array}$ & $\begin{array}{c}\text { Follow } \\
\text { 10.2.3.4.7.2 for } \\
\text { lengthy detailed } \\
\text { behavior }\end{array}$ & 10.2.3.4.7.2 \\
\hline
\end{tabular}

\subsubsection{Call upgrade from basic to emergency group call}

In this scenario, a basic group call is ongoing. A user decides to upgrade the call to an emergency group call, so other users’ call types are upgraded accordingly.

Scenario purpose: To observe that a basic group call can be upgraded to an emergency group call.

Table 40: Call upgrade from basic to emergency group call

\begin{tabular}{|c|c|c|c|c|c|c|c|}
\hline & Timeline & $\begin{array}{c}\text { Triggering } \\
\text { event }\end{array}$ & $\begin{array}{c}\text { UE A state } \\
\text { transition }\end{array}$ & $\begin{array}{c}\text { UE B state } \\
\text { transition }\end{array}$ & $\begin{array}{c}\text { UE C state } \\
\text { transition }\end{array}$ & $\begin{array}{c}\text { Consequent } \\
\text { behavior }\end{array}$ & $\begin{array}{c}\text { Reference } \\
\text { for details }\end{array}$ \\
\hline 0 & - & - & $\begin{array}{c}\text { T2: in- } \\
\text { progress } \\
\text { basic group } \\
\text { call }\end{array}$ & $\begin{array}{c}\text { T2: in- } \\
\text { progress } \\
\text { basic } \\
\text { group call }\end{array}$ & $\begin{array}{c}\text { T2: in- } \\
\text { progress } \\
\text { basic } \\
\text { group call }\end{array}$ & - & - \\
\hline
\end{tabular}




\begin{tabular}{|c|c|c|c|c|c|c|c|}
\hline & Timeline & $\begin{array}{c}\text { Triggering } \\
\text { event }\end{array}$ & $\begin{array}{l}\text { UE A state } \\
\text { transition }\end{array}$ & $\begin{array}{l}\text { UE B state } \\
\text { transition }\end{array}$ & $\begin{array}{l}\text { UE C state } \\
\text { transition }\end{array}$ & $\begin{array}{c}\text { Consequent } \\
\text { behavior }\end{array}$ & $\begin{array}{l}\text { Reference } \\
\text { for details }\end{array}$ \\
\hline 1 & T0 & $\begin{array}{l}\text { UE A } \\
\text { requests to } \\
\text { upgrade the } \\
\text { call to } \\
\text { "EMERGE } \\
\text { NCY } \\
\text { GROUP } \\
\text { CALL" }\end{array}$ & $\begin{array}{l}\text { T1: in- } \\
\text { progress } \\
\text { emergency } \\
\text { group call }\end{array}$ & - & - & $\begin{array}{c}\text { UE A sets } \\
\text { current call type } \\
\text { accordingly as } \\
\text { well as current } \\
\text { ProSe per-packet } \\
\text { priority, starts } \\
\text { TFG13, stores } \\
\text { UTC time as last } \\
\text { call type change } \\
\text { time of the call as } \\
\text { well as own } \\
\text { MCPTT user ID } \\
\text { as last user to } \\
\text { change call type } \\
\text { of the call, and } \\
\text { generates and } \\
\text { sends GROUP } \\
\text { CALL } \\
\text { ANNOUNCEME } \\
\text { NT }\end{array}$ & 10.2.3.4.7.1 \\
\hline 2 & $\begin{array}{c}\mathrm{T} 0+ \\
\mathrm{t} \_\mathrm{AB} 1\end{array}$ & $\begin{array}{l}\text { UE B and } \\
\text { UE C } \\
\text { receive } \\
\text { GROUP } \\
\text { CALL } \\
\text { ANNOUN } \\
\text { CEMENT }\end{array}$ & - & $\begin{array}{l}\text { T1: in- } \\
\text { progress } \\
\text { emergency } \\
\text { group call }\end{array}$ & $\begin{array}{l}\text { T: in- } \\
\text { progress } \\
\text { emergency } \\
\text { group call }\end{array}$ & $\begin{array}{c}\text { Follow } \\
\text { 10.2.3.4.7.2 for } \\
\text { lengthy detailed } \\
\text { behavior }\end{array}$ & 10.2.3.4.7.2 \\
\hline
\end{tabular}

\subsubsection{Call upgrade from imminent peril group call}

In this scenario, an imminent peril group call is ongoing. A user decides to upgrade the call to an emergency group call, and the other users' call types are upgraded accordingly.

Scenario purpose: To observe that an imminent peril group call can be upgraded to an emergency group call.

Table 41: Call upgrade from imminent peril group call

\begin{tabular}{|c|c|c|c|c|c|c|c|}
\hline & Timeline & $\begin{array}{c}\text { Triggering } \\
\text { event }\end{array}$ & $\begin{array}{c}\text { UE A state } \\
\text { transition }\end{array}$ & $\begin{array}{c}\text { UE B state } \\
\text { transition }\end{array}$ & $\begin{array}{c}\text { UE C state } \\
\text { transition }\end{array}$ & $\begin{array}{c}\text { Consequent } \\
\text { behavior }\end{array}$ & $\begin{array}{c}\text { Reference } \\
\text { for details }\end{array}$ \\
\hline 0 & - & - & $\begin{array}{c}\text { T3: in- } \\
\text { progress } \\
\text { imminent } \\
\text { peril group } \\
\text { call }\end{array}$ & $\begin{array}{c}\text { T3: in- } \\
\text { progress } \\
\text { imminent } \\
\text { peril } \\
\text { group call }\end{array}$ & $\begin{array}{c}\text { T3: in- } \\
\text { progress } \\
\text { imminent } \\
\text { peril } \\
\text { group call }\end{array}$ & - & - \\
\hline
\end{tabular}




\begin{tabular}{|c|c|c|c|c|c|c|c|}
\hline & Timeline & $\begin{array}{c}\text { Triggering } \\
\text { event }\end{array}$ & $\begin{array}{l}\text { UE A state } \\
\text { transition }\end{array}$ & $\begin{array}{l}\text { UE B state } \\
\text { transition }\end{array}$ & $\begin{array}{l}\text { UE C state } \\
\text { transition }\end{array}$ & $\begin{array}{c}\text { Consequent } \\
\text { behavior }\end{array}$ & $\begin{array}{l}\text { Reference } \\
\text { for details }\end{array}$ \\
\hline 1 & T0 & $\begin{array}{l}\text { UE A } \\
\text { requests to } \\
\text { upgrade the } \\
\text { call to } \\
\text { "EMERGE } \\
\text { NCY } \\
\text { GROUP } \\
\text { CALL" }\end{array}$ & $\begin{array}{l}\text { T1: in- } \\
\text { progress } \\
\text { emergency } \\
\text { group call }\end{array}$ & - & - & $\begin{array}{c}\text { UE A sets } \\
\text { current call type } \\
\text { accordingly as } \\
\text { well as current } \\
\text { ProSe per- } \\
\text { packet priority, } \\
\text { starts TFG13 } \\
\text { and stops } \\
\text { TFG14, stores } \\
\text { UTC time as } \\
\text { last call type } \\
\text { change time of } \\
\text { the call as well } \\
\text { as own MCPTT } \\
\text { user ID as last } \\
\text { user to change } \\
\text { call type of the } \\
\text { call, generates } \\
\text { and sends } \\
\text { GROUP CALL } \\
\text { ANNOUNCEM } \\
\text { ENT }\end{array}$ & 10.2.3.4.7.1 \\
\hline 2 & $\begin{array}{l}\mathrm{T} 0+ \\
\mathrm{t} \_\mathrm{AB} 1\end{array}$ & $\begin{array}{l}\text { UE B and } \\
\text { UE C } \\
\text { receive } \\
\text { GROUP } \\
\text { CALL } \\
\text { ANNOUNC } \\
\text { EMENT }\end{array}$ & - & $\begin{array}{l}\text { T1: in- } \\
\text { progress } \\
\text { emergency } \\
\text { group call }\end{array}$ & $\begin{array}{l}\text { T1: in- } \\
\text { progress } \\
\text { emergency } \\
\text { group call }\end{array}$ & $\begin{array}{c}\text { Follow } \\
\text { 10.2.3.4.7.2 for } \\
\text { lengthy detailed } \\
\text { behavior }\end{array}$ & 10.2.3.4.7.2 \\
\hline
\end{tabular}

\subsubsection{Call type downgrade}

\subsubsection{Explicit downgrade from emergency call}

In this scenario, an emergency group call is in progress. An authorized user (UE A) decides to downgrade the call to a basic group call. In addition, multiple TFG11 expirations are observed in this test scenario. It is assumed that if there are n "GROUP CALL EMERGENCY END" messages, then the first $\mathrm{n}-1$ are lost, and UE $B$ and UE C only receive the $\mathrm{n}^{\text {th }}$ message.

Scenario purpose: To observe that an emergency group call can explicitly be downgraded to a basic group call. 
Table 42: Explicit downgrade from emergency call

\begin{tabular}{|c|c|c|c|c|c|c|c|}
\hline & Timeline & $\begin{array}{c}\text { Triggering } \\
\text { event }\end{array}$ & $\begin{array}{l}\text { UE A state } \\
\text { transition }\end{array}$ & $\begin{array}{l}\text { UE B state } \\
\text { transition }\end{array}$ & $\begin{array}{l}\text { UE C state } \\
\text { transition }\end{array}$ & $\begin{array}{c}\text { Consequent } \\
\text { behavior }\end{array}$ & $\begin{array}{l}\text { Reference } \\
\text { for details }\end{array}$ \\
\hline 0 & - & - & $\begin{array}{c}\text { T1: in- } \\
\text { progress } \\
\text { emergency } \\
\text { group call }\end{array}$ & $\begin{array}{c}\text { T1: in- } \\
\text { progress } \\
\text { emergency } \\
\text { group call }\end{array}$ & $\begin{array}{c}\text { T1: in- } \\
\text { progress } \\
\text { emergency } \\
\text { group call }\end{array}$ & - & - \\
\hline 1 & T0 & $\begin{array}{c}\text { UE A } \\
\text { requests to } \\
\text { downgrade } \\
\text { the call }\end{array}$ & $\begin{array}{l}\text { T2: in- } \\
\text { progress } \\
\text { basic } \\
\text { group call }\end{array}$ & - & - & $\begin{array}{l}\text { UE A sets } \\
\text { stored current } \\
\text { call type to } \\
\text { "BASIC } \\
\text { GROUP } \\
\text { CALL” as well } \\
\text { as per-packet } \\
\text { priority, call } \\
\text { type change } \\
\text { time and last } \\
\text { user to change } \\
\text { call type } \\
\text { accordingly, } \\
\text { generates and } \\
\text { sends GROUP } \\
\text { CALL } \\
\text { EMERGENCY } \\
\text { END, stops } \\
\text { TFG13, starts } \\
\text { TFG11 and } \\
\text { initializes } \\
\text { CFG11 }\end{array}$ & 10.2.3.4.8.1 \\
\hline 2 & $\begin{array}{c}\text { T0 + } \\
\text { TFG11 }\end{array}$ & $\begin{array}{c}\text { UE A } \\
\text { TFG11 } \\
\text { expires }\end{array}$ & - & - & - & $\begin{array}{l}\text { UE A generates } \\
\text { and sends } \\
\text { GROUP CALL } \\
\text { EMERGENCY } \\
\text { END, } \\
\text { increments } \\
\text { value of } \\
\text { CFG11, and } \\
\text { starts TFG11 (if } \\
\text { value of CFG11 } \\
\text { is less than its } \\
\text { upper limit) } \\
\end{array}$ & 10.2.3.4.8.2 \\
\hline 3 & $\begin{array}{c}\mathrm{T} 0+ \\
\mathrm{n} * \mathrm{TFG} 1 \\
1\end{array}$ & $\begin{array}{c}\text { UE A } \\
\text { TFG11 } \\
\text { expires } \\
\text { (CFG11 at } \\
\text { its upper } \\
\text { limit) }\end{array}$ & - & - & - & $\begin{array}{l}\text { UE A generates } \\
\text { and sends } \\
\text { GROUP CALL } \\
\text { EMERGENCY } \\
\text { END, and } \\
\text { increments } \\
\text { value of CFG11 } \\
\text { (now equal to } \\
\text { its upper limit) }\end{array}$ & 10.2.3.4.8.2 \\
\hline
\end{tabular}




\begin{tabular}{|c|c|c|c|c|c|c|c|}
\hline & Timeline & $\begin{array}{c}\text { Triggering } \\
\text { event }\end{array}$ & $\begin{array}{l}\text { UE A state } \\
\text { transition }\end{array}$ & $\begin{array}{l}\text { UE B state } \\
\text { transition }\end{array}$ & $\begin{array}{l}\text { UE C state } \\
\text { transition }\end{array}$ & $\begin{array}{c}\text { Consequent } \\
\text { behavior }\end{array}$ & $\begin{array}{l}\text { Reference } \\
\text { for details }\end{array}$ \\
\hline 4 & $\begin{array}{c}\mathrm{T} 0+ \\
\mathrm{n}^{*} \mathrm{TFG} 1 \\
1+ \\
\mathrm{t} \_\mathrm{AB} 1\end{array}$ & $\begin{array}{l}\text { UE B and } \\
\text { UE C } \\
\text { receive } \\
\text { GROUP } \\
\text { CALL } \\
\text { EMERGE } \\
\text { NCY END }\end{array}$ & - & $\begin{array}{c}\text { T2: in- } \\
\text { progress } \\
\text { basic group } \\
\text { call }\end{array}$ & $\begin{array}{l}\text { T2: in- } \\
\text { progress } \\
\text { basic group } \\
\text { call }\end{array}$ & $\begin{array}{l}\text { UE B and UE C } \\
\text { set stored last } \\
\text { call type change } \\
\text { time and last } \\
\text { user to change } \\
\text { call type to } \\
\text { corresponding } \\
\text { values of } \\
\text { GROUP CALL } \\
\text { EMERGENCY } \\
\text { END message, } \\
\text { set stored } \\
\text { current call type } \\
\text { to "BASIC } \\
\text { GROUP } \\
\text { CALL" as well } \\
\text { as current ProSe } \\
\text { per-packet } \\
\text { priority to } \\
\text { corresponding } \\
\text { value, and stop } \\
\text { TFG13 }\end{array}$ & 10.2.3.4.8.3 \\
\hline
\end{tabular}

\subsubsection{Explicit downgrade from imminent peril call}

In this scenario, an imminent peril group call is in progress. An authorized user (UE A) decides to downgrade the call. In addition, multiple TFG12 expirations are observed in this test scenario. It is assumed that if there are n "GROUP CALL IMMINENT PERIL END" messages, then the first $n-1$ are lost, thus UE B and UE C only receive the $n^{\text {th }}$ message.

Scenario purpose: To observe that an imminent peril group call can explicitly be downgraded to a basic group call.

Table 43: Explicit downgrade from imminent peril call

\begin{tabular}{|c|c|c|c|c|c|c|c|}
\hline & Timeline & $\begin{array}{c}\text { Triggering } \\
\text { event }\end{array}$ & $\begin{array}{c}\text { UE A state } \\
\text { transition }\end{array}$ & $\begin{array}{c}\text { UE B state } \\
\text { transition }\end{array}$ & $\begin{array}{c}\text { UE C state } \\
\text { transition }\end{array}$ & $\begin{array}{c}\text { Consequent } \\
\text { behavior }\end{array}$ & $\begin{array}{c}\text { Reference } \\
\text { for details }\end{array}$ \\
\hline 0 & & & $\begin{array}{c}\text { T3: in- } \\
\text { progress } \\
\text { imminent } \\
\text { peril group } \\
\text { call }\end{array}$ & $\begin{array}{c}\text { T3: in- } \\
\text { progress } \\
\text { imminent } \\
\text { peril group } \\
\text { call }\end{array}$ & $\begin{array}{c}\text { T3: in- } \\
\text { progress } \\
\text { imminent } \\
\text { peril } \\
\text { group call }\end{array}$ & - & - \\
\hline
\end{tabular}




\begin{tabular}{|c|c|c|c|c|c|c|c|}
\hline & Timeline & $\begin{array}{c}\text { Triggering } \\
\text { event }\end{array}$ & $\begin{array}{l}\text { UE A state } \\
\text { transition }\end{array}$ & $\begin{array}{l}\text { UE B state } \\
\text { transition }\end{array}$ & $\begin{array}{l}\text { UE C state } \\
\text { transition }\end{array}$ & $\begin{array}{c}\text { Consequent } \\
\text { behavior }\end{array}$ & $\begin{array}{l}\text { Reference } \\
\text { for details }\end{array}$ \\
\hline 1 & T0 & $\begin{array}{c}\text { UE A } \\
\text { requests to } \\
\text { downgrade } \\
\text { the call }\end{array}$ & $\begin{array}{c}\text { T2: in- } \\
\text { progress basic } \\
\text { group call }\end{array}$ & - & - & $\begin{array}{l}\text { UE A sets } \\
\text { stored } \\
\text { current call } \\
\text { type to } \\
\text { "BASIC } \\
\text { GROUP } \\
\text { CALL" as } \\
\text { well as per- } \\
\text { packet } \\
\text { priority, call } \\
\text { type change } \\
\text { time and last } \\
\text { user to } \\
\text { change call } \\
\text { type } \\
\text { accordingly, } \\
\text { generates } \\
\text { and sends } \\
\text { GROUP } \\
\text { CALL } \\
\text { IMMINENT } \\
\text { PERIL } \\
\text { END, stops } \\
\text { TFG14, } \\
\text { starts } \\
\text { TFG12 and } \\
\text { initializes } \\
\text { CFG12 }\end{array}$ & 10.2 .3 .4 .8 .4 \\
\hline 2 & $\begin{array}{c}\text { T0 + } \\
\text { TFG12 }\end{array}$ & $\begin{array}{c}\text { UE A } \\
\text { TFG12 } \\
\text { expires }\end{array}$ & - & - & - & $\begin{array}{c}\text { UE A } \\
\text { generates } \\
\text { and sends } \\
\text { GROUP } \\
\text { CALL } \\
\text { IMMINENT } \\
\text { PERIL } \\
\text { END, } \\
\text { increments } \\
\text { value of } \\
\text { CFG12, and } \\
\text { starts } \\
\text { TFG12 } \\
\text { (because } \\
\text { value of } \\
\text { CFG12 is } \\
\text { less than its } \\
\text { upper limit) }\end{array}$ & 10.2 .3 .4 .8 .5 \\
\hline
\end{tabular}




\begin{tabular}{|c|c|c|c|c|c|c|c|}
\hline & Timeline & $\begin{array}{c}\text { Triggering } \\
\text { event }\end{array}$ & $\begin{array}{l}\text { UE A state } \\
\text { transition }\end{array}$ & $\begin{array}{l}\text { UE B state } \\
\text { transition }\end{array}$ & $\begin{array}{l}\text { UE C state } \\
\text { transition }\end{array}$ & $\begin{array}{c}\text { Consequent } \\
\text { behavior }\end{array}$ & $\begin{array}{l}\text { Reference } \\
\text { for details }\end{array}$ \\
\hline 3 & $\begin{array}{c}\text { T0 + } \\
\text { n*TFG12 }\end{array}$ & $\begin{array}{c}\text { UE A } \\
\text { TFG12 } \\
\text { (with } \\
\text { CFG12 at } \\
\text { upper limit } \\
\text { - 1) }\end{array}$ & - & - & - & $\begin{array}{c}\text { UE A } \\
\text { generates } \\
\text { and sends } \\
\text { GROUP } \\
\text { CALL } \\
\text { IMMINENT } \\
\text { PERIL } \\
\text { END, and } \\
\text { increments } \\
\text { value of } \\
\text { CFG12 }\end{array}$ & 10.2 .3 .4 .8 .5 \\
\hline 4 & $\begin{array}{c}\text { T0 + } \\
\mathrm{n}^{*} \mathrm{TFG} 12 \\
+\mathrm{t} \_\mathrm{AB} 1\end{array}$ & $\begin{array}{l}\text { UE B and } \\
\text { UE C } \\
\text { receive } \\
\text { GROUP } \\
\text { CALL } \\
\text { IMMINEN } \\
\text { T PERIL } \\
\text { END }\end{array}$ & - & $\begin{array}{l}\text { T2: in- } \\
\text { progress } \\
\text { basic group } \\
\text { call }\end{array}$ & $\begin{array}{l}\text { T2: in- } \\
\text { progress } \\
\text { basic } \\
\text { group call }\end{array}$ & $\begin{array}{c}\text { UE B and } \\
\text { UE C set } \\
\text { stored last } \\
\text { call type } \\
\text { change time } \\
\text { and last user } \\
\text { to change } \\
\text { call type to } \\
\text { correspondi } \\
\text { ng values of } \\
\text { GROUP } \\
\text { CALL } \\
\text { IMMINENT } \\
\text { PERIL END } \\
\text { message, set } \\
\text { stored } \\
\text { current call } \\
\text { type to } \\
\text { "BASIC } \\
\text { GROUP } \\
\text { CALL" as } \\
\text { well as } \\
\text { current } \\
\text { ProSe per- } \\
\text { packet } \\
\text { priority to } \\
\text { correspondi } \\
\text { ng value, } \\
\text { and stop } \\
\text { TFG14 }\end{array}$ & 10.2 .3 .4 .8 .6 \\
\hline
\end{tabular}

\subsubsection{Implicit downgrade from emergency call}

In this scenario, an emergency group call is in progress. The expiration of a user's timer, TFG13, invokes the implicit downgrade of the call type.

Scenario purpose: To observe that an emergency group call can implicitly (timer expiration) initiate the downgrade to a basic group call. 
Table 44: Implicit downgrade from emergency call

\begin{tabular}{|c|c|c|c|c|c|c|c|}
\hline & Timeline & $\begin{array}{c}\text { Triggering } \\
\text { event }\end{array}$ & $\begin{array}{l}\text { UE A state } \\
\text { transition }\end{array}$ & $\begin{array}{l}\text { UE B state } \\
\text { transition }\end{array}$ & $\begin{array}{l}\text { UE C state } \\
\text { transition }\end{array}$ & $\begin{array}{c}\text { Consequent } \\
\text { behavior }\end{array}$ & $\begin{array}{l}\text { Reference } \\
\text { for details }\end{array}$ \\
\hline 0 & - & $2-2$ & $\begin{array}{c}\text { T1: in- } \\
\text { progress } \\
\text { emergency } \\
\text { group call }\end{array}$ & $\begin{array}{c}\text { T1: in- } \\
\text { progress } \\
\text { emergency } \\
\text { group call }\end{array}$ & $\begin{array}{c}\text { T1: in- } \\
\text { progress } \\
\text { emergency } \\
\text { group call }\end{array}$ & 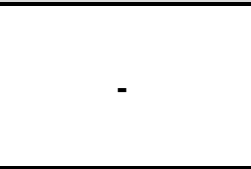 & - \\
\hline 1 & T0 & $\begin{array}{c}\text { UE A } \\
\text { TFG13 } \\
\text { expires }\end{array}$ & $\begin{array}{l}\text { T2: in- } \\
\text { progress } \\
\text { basic group } \\
\text { call }\end{array}$ & - & - & $\begin{array}{l}\text { UE A sets } \\
\text { stored current } \\
\text { call type to } \\
\text { "BASIC } \\
\text { GROUP CALL" } \\
\text { as well as } \\
\text { current ProSe } \\
\text { per-packet } \\
\text { priority to } \\
\text { corresponding } \\
\text { value, and sets } \\
\text { current UTC } \\
\text { time as last call } \\
\text { type change } \\
\text { time of the call } \\
\text { and own } \\
\text { MCPTT user ID } \\
\text { as last user to } \\
\text { change call type } \\
\text { of the call }\end{array}$ & 10.2 .3 .4 .8 .8 \\
\hline
\end{tabular}

\subsubsection{Implicit downgrade from imminent peril call}

In this scenario, an imminent peril group call is in progress. The expiration of a user's timer, TFG14, invokes the downgrade of the call type.

Scenario purpose: To observe that an imminent peril group call can implicitly (timer expiration) initiate the downgrade to a basic group call.

Table 45: Implicit downgrade from imminent peril call

\begin{tabular}{|c|c|c|c|c|c|c|c|}
\hline & Timeline & $\begin{array}{c}\text { Triggering } \\
\text { event }\end{array}$ & $\begin{array}{c}\text { UE A state } \\
\text { transition }\end{array}$ & $\begin{array}{c}\text { UE B state } \\
\text { transition }\end{array}$ & $\begin{array}{c}\text { UE C state } \\
\text { transition }\end{array}$ & $\begin{array}{c}\text { Consequent } \\
\text { behavior }\end{array}$ & $\begin{array}{c}\text { Reference } \\
\text { for details }\end{array}$ \\
\hline 0 & & & $\begin{array}{c}\text { T3: in- } \\
\text { progress } \\
\text { imminent } \\
\text { peril } \\
\text { group call }\end{array}$ & $\begin{array}{c}\text { T3: in- } \\
\text { progress } \\
\text { imminent } \\
\text { peril } \\
\text { group call }\end{array}$ & $\begin{array}{c}\text { T3: in- } \\
\text { progress } \\
\text { imminent } \\
\text { peril group } \\
\text { call }\end{array}$ & & \\
\end{tabular}




\begin{tabular}{|c|c|c|c|c|c|c|c|}
\hline & Timeline & $\begin{array}{c}\text { Triggering } \\
\text { event }\end{array}$ & $\begin{array}{l}\text { UE A state } \\
\text { transition }\end{array}$ & $\begin{array}{l}\text { UE B state } \\
\text { transition }\end{array}$ & $\begin{array}{c}\text { UE C state } \\
\text { transition }\end{array}$ & $\begin{array}{c}\text { Consequent } \\
\text { behavior }\end{array}$ & $\begin{array}{l}\text { Reference } \\
\text { for details }\end{array}$ \\
\hline 1 & T0 & $\begin{array}{c}\text { UE A } \\
\text { TFG14 } \\
\text { expires }\end{array}$ & $\begin{array}{c}\text { T2: in- } \\
\text { progress } \\
\text { basic } \\
\text { group call }\end{array}$ & - & - & $\begin{array}{l}\text { UE A sets stored } \\
\text { current call type to } \\
\text { "BASIC GROUP } \\
\text { CALL" as well as } \\
\text { current ProSe per- } \\
\text { packet priority to } \\
\text { corresponding } \\
\text { value, and sets } \\
\text { current UTC time as } \\
\text { last call type change } \\
\text { time of the call and } \\
\text { own MCPTT user } \\
\text { ID as last user to } \\
\text { change call type of } \\
\text { the call }\end{array}$ & 10.2 .3 .4 .8 .9 \\
\hline
\end{tabular}

\subsubsection{Call release}

\subsubsection{Call release after call establishment}

In this scenario, a group call is ongoing (any call type state). One of the users releases the call.

Scenario purpose: To observe that a user can release the call, regardless of the ongoing call type state.

Table 46: Call release after call establishment

\begin{tabular}{|c|c|c|c|c|c|c|c|}
\hline & Timeline & $\begin{array}{c}\text { Triggering } \\
\text { event }\end{array}$ & $\begin{array}{l}\text { UE A state } \\
\text { transition }\end{array}$ & $\begin{array}{l}\text { UE B state } \\
\text { transition }\end{array}$ & $\begin{array}{l}\text { UE C state } \\
\text { transition }\end{array}$ & $\begin{array}{c}\text { Consequent } \\
\text { behavior }\end{array}$ & $\begin{array}{l}\text { Reference } \\
\text { for details }\end{array}$ \\
\hline 0 & - & - & $\begin{array}{c}\text { T2: in- } \\
\text { progress } \\
\text { basic, T3: } \\
\text { imminent } \\
\text { peril or T1: } \\
\text { emergency } \\
\text { group call } \\
\text { (same call } \\
\text { type state as } \\
\text { UE B and UE } \\
\text { C) }\end{array}$ & $\begin{array}{c}\text { T2: in- } \\
\text { progress } \\
\text { basic, T3: } \\
\text { imminent } \\
\text { peril or T1: } \\
\text { emergency } \\
\text { group call } \\
\text { (same call } \\
\text { type state as } \\
\text { UE A and UE } \\
\text { C) }\end{array}$ & $\begin{array}{c}\text { T2: in- } \\
\text { progress } \\
\text { basic, T3: } \\
\text { imminent } \\
\text { peril or T1: } \\
\text { emergency } \\
\text { group call } \\
\text { (same call } \\
\text { type state as } \\
\text { UE A and } \\
\text { UE B) }\end{array}$ & - & - \\
\hline 1 & T0 & $\begin{array}{l}\text { UE A } \\
\text { requests to } \\
\text { release the } \\
\text { call }\end{array}$ & $\begin{array}{l}\text { T0: waiting } \\
\text { for call to } \\
\text { establish }\end{array}$ & - & - & $\begin{array}{c}\text { UE A releases } \\
\text { stored current } \\
\text { call type, ProSe } \\
\text { per-packet } \\
\text { priority, Last } \\
\text { call type } \\
\text { change time } \\
\text { and Last user } \\
\text { to change call } \\
\text { type }\end{array}$ & 10.2 .3 .4 .10 \\
\hline
\end{tabular}




\subsubsection{Call release before call establishment}

In this scenario, there is no ongoing group call yet. One of the users attempts to establish a call but releases it before completion.

Scenario purpose: To observe that a user attempting to establish a call can release from the call prior to completion.

Table 47: Call release before call establishment

\begin{tabular}{|c|c|c|c|c|c|c|c|}
\hline & Timeline & $\begin{array}{c}\text { Triggering } \\
\text { event }\end{array}$ & $\begin{array}{c}\text { UE A state } \\
\text { transition }\end{array}$ & $\begin{array}{c}\text { UE B state } \\
\text { transition }\end{array}$ & $\begin{array}{c}\text { UE C state } \\
\text { transition }\end{array}$ & $\begin{array}{c}\text { Consequent } \\
\text { behavior }\end{array}$ & $\begin{array}{c}\text { Reference } \\
\text { for details }\end{array}$ \\
\hline 0 & - & - & $\begin{array}{c}\text { T0: } \\
\text { waiting for } \\
\text { call to } \\
\text { establish }\end{array}$ & $\begin{array}{c}\text { T0: } \\
\text { waiting for } \\
\text { call to } \\
\text { establish }\end{array}$ & $\begin{array}{c}\text { T0: } \\
\text { waiting for } \\
\text { call to } \\
\text { establish }\end{array}$ & - & - \\
\hline 1 & T0 & $\begin{array}{c}\text { UE A } \\
\text { requests to } \\
\text { reject the } \\
\text { call }\end{array}$ & - & - & $\begin{array}{c}\text { UE A releases stored } \\
\text { current call type, } \\
\text { ProSe per-packet } \\
\text { priority, Last call } \\
\text { type change time and } \\
\text { Last user to change } \\
\text { call type }\end{array}$ & $\mathbf{1 0 . 2 . 3 . 4 . 1 1}$ \\
\hline
\end{tabular}

\subsubsection{Call merge}

\subsubsection{Call merge from different call type states}

In this scenario, there are two ongoing group calls, (UE A and UE B) and (UE C), both group calls with the same group ID, but different call type states. UE A and UE B receive a group call announcement from UE C, and adjust their call type status accordingly.

Scenario purpose: To observe that the call type states are properly updated after the merge.

Table 48: Call merge from different call type states

\begin{tabular}{|c|c|c|c|c|c|c|c|}
\hline & Timeline & $\begin{array}{c}\text { Triggering } \\
\text { event }\end{array}$ & $\begin{array}{c}\text { UE A state } \\
\text { transition }\end{array}$ & $\begin{array}{c}\text { UE B state } \\
\text { transition }\end{array}$ & $\begin{array}{c}\text { UE C state } \\
\text { transition }\end{array}$ & $\begin{array}{c}\text { Consequent } \\
\text { behavior }\end{array}$ & $\begin{array}{c}\text { Reference } \\
\text { for details }\end{array}$ \\
\hline 0 & & & $\begin{array}{c}\text { T3: in- } \\
\text { progress } \\
\text { imminent } \\
\text { peril group } \\
\text { call }\end{array}$ & $\begin{array}{c}\text { T3: in- } \\
\text { progress } \\
\text { imminent } \\
\text { peril group } \\
\text { call }\end{array}$ & $\begin{array}{c}\text { T1: in- } \\
\text { progress } \\
\text { emergency } \\
\text { group call }\end{array}$ & - & - \\
\hline
\end{tabular}




\begin{tabular}{|c|c|c|c|c|c|c|c|}
\hline & Timeline & $\begin{array}{c}\text { Triggering } \\
\text { event }\end{array}$ & $\begin{array}{l}\text { UE A state } \\
\text { transition }\end{array}$ & $\begin{array}{l}\text { UE B state } \\
\text { transition }\end{array}$ & $\begin{array}{c}\text { UE C state } \\
\text { transition }\end{array}$ & $\begin{array}{c}\text { Consequent } \\
\text { behavior }\end{array}$ & $\begin{array}{l}\text { Reference } \\
\text { for details }\end{array}$ \\
\hline 1 & T0 & $\begin{array}{c}\text { UE C TFG2 } \\
\text { expires }\end{array}$ & - & - & - & $\begin{array}{c}\text { UE C sends } \\
\text { GROUP } \\
\text { CALL } \\
\text { ANNOUNCE } \\
\text { MENT } \\
\end{array}$ & $\begin{array}{r}\text { NOTE: } \\
\text { happens } \\
\text { outside } \\
\text { call type } \\
\text { control }\end{array}$ \\
\hline 2 & $\begin{array}{l}\mathrm{T} 0+ \\
\mathrm{t} \_\mathrm{CA} 1\end{array}$ & $\begin{array}{c}\text { UE A and } \\
\text { UE B } \\
\text { receive } \\
\text { GROUP } \\
\text { CALL } \\
\text { ANNOUNC } \\
\text { EMENT } \\
\text { message }\end{array}$ & $\begin{array}{l}\text { T1: in- } \\
\text { progress } \\
\text { emergency } \\
\text { group call }\end{array}$ & $\begin{array}{l}\text { T1: in- } \\
\text { progress } \\
\text { emergency } \\
\text { group call }\end{array}$ & - & $\begin{array}{c}\text { Follow } \\
\text { 10.2.3.4.9 for } \\
\text { lengthy } \\
\text { detailed } \\
\text { behavior }\end{array}$ & 10.2.3.4.9 \\
\hline
\end{tabular}

\subsubsection{Call merge from same call type state}

In this scenario, there are two ongoing group calls with the same group ID and same call type state, but one group call (UE C) has a lower call start time IE than the other group (UE A and UE B). A call merge occurs for UE A and UE B when each receive a GROUP CALL ANNOUNCEMENT from UE $C$. There is no state change, just updates to the internal variables.

Scenario purpose: To observe that the call type internal variables are properly updated after the merge.

Table 49: Call merge from same call type state

\begin{tabular}{|c|c|c|c|c|c|c|c|}
\hline & Timeline & $\begin{array}{c}\text { Triggering } \\
\text { event }\end{array}$ & $\begin{array}{l}\text { UE A state } \\
\text { transition }\end{array}$ & $\begin{array}{l}\text { UE B state } \\
\text { transition }\end{array}$ & $\begin{array}{c}\text { UE C state } \\
\text { transition }\end{array}$ & $\begin{array}{c}\text { Consequent } \\
\text { behavior }\end{array}$ & $\begin{array}{l}\text { Reference } \\
\text { for details }\end{array}$ \\
\hline 0 & - & - & $\begin{array}{l}\text { T1: in- } \\
\text { progress } \\
\text { emergency } \\
\text { group call }\end{array}$ & $\begin{array}{l}\text { T1: in- } \\
\text { progress } \\
\text { emergency } \\
\text { group call }\end{array}$ & $\begin{array}{l}\text { T1: in- } \\
\text { progress } \\
\text { emergency } \\
\text { group call }\end{array}$ & - & - \\
\hline 1 & T0 & $\begin{array}{c}\text { UE C TFG2 } \\
\text { expires }\end{array}$ & - & - & - & $\begin{array}{c}\text { UE C sends } \\
\text { GROUP } \\
\text { CALL } \\
\text { ANNOUNCE } \\
\text { MENT } \\
\end{array}$ & $\begin{array}{r}\text { NOTE: } \\
\text { happens } \\
\text { outside } \\
\text { call type } \\
\text { control } \\
\end{array}$ \\
\hline
\end{tabular}




\begin{tabular}{|c|c|c|c|c|c|c|c|}
\hline & Timeline & $\begin{array}{c}\text { Triggering } \\
\text { event }\end{array}$ & $\begin{array}{l}\text { UE A state } \\
\text { transition }\end{array}$ & $\begin{array}{l}\text { UE B state } \\
\text { transition }\end{array}$ & $\begin{array}{l}\text { UE C state } \\
\text { transition }\end{array}$ & $\begin{array}{c}\text { Consequent } \\
\text { behavior }\end{array}$ & $\begin{array}{l}\text { Reference } \\
\text { for details }\end{array}$ \\
\hline 2 & $\begin{array}{l}\mathrm{T} 0+ \\
\mathrm{t} \_\mathrm{CA} 1\end{array}$ & $\begin{array}{l}\text { UE A and } \\
\text { UE B } \\
\text { receive } \\
\text { GROUP } \\
\text { CALL } \\
\text { ANNOUNC } \\
\text { EMENT } \\
\text { message }\end{array}$ & - & - & - & $\begin{array}{l}\text { UE A and } \\
\text { UE B store } \\
\text { the value of } \\
\text { the Last call } \\
\text { type change } \\
\text { time IE, store } \\
\text { the value of } \\
\text { the Last user } \\
\text { to change call } \\
\text { type IE, and } \\
\text { set PPPP to } \\
\text { value for } \\
\text { emergency } \\
\text { group call }\end{array}$ & 10.2.3.4.9 \\
\hline
\end{tabular}

The scenario can be generalized similarly to the situation when all three UEs have the same group ID, the same Call Type and the same call start time, but the call identifier IE of UE C's group call is lower than that of the group call that UE A and UE B are in. 


\section{Test scenarios details - broadcast group call}

According to 3GPP TS 24.379 v14.2.0 [4], a broadcast group call is "a group call where the initiating MCPTT user expects no response from the other MCPTT users, so that when the user's transmission is complete, so is the call." No floor control procedure is specified here, since it is assumed that there shall be no change in floor arbitration throughout the entire duration of the broadcast group call.

\section{1. $\quad$ Broadcast group call - call control}

\subsubsection{Call setup}

\subsubsection{Call setup - establish a new call}

In this scenario a user, UE A, decides to establish a broadcast group call (no existing one yet). UE B requires user acknowledgement, while UE C does not.

Scenario purpose: To observe that a new broadcast group call can be established and that users can join in.

Table 50: Call setup - establish a new call

\begin{tabular}{|c|c|c|c|c|c|c|c|}
\hline & Timeline & $\begin{array}{c}\text { Triggering } \\
\text { event }\end{array}$ & $\begin{array}{l}\text { UE A state } \\
\text { transition }\end{array}$ & $\begin{array}{l}\text { UE B state } \\
\text { transition }\end{array}$ & $\begin{array}{l}\text { UE C state } \\
\text { transition }\end{array}$ & $\begin{array}{c}\text { Consequent } \\
\text { behavior }\end{array}$ & $\begin{array}{l}\text { Reference } \\
\text { for } \\
\text { details }\end{array}$ \\
\hline 0 & - & - & $\begin{array}{l}\text { B1: start- } \\
\text { stop }\end{array}$ & $\begin{array}{l}\text { B1: start- } \\
\text { stop }\end{array}$ & $\begin{array}{l}\text { B1: start- } \\
\text { stop }\end{array}$ & - & - \\
\hline 1 & T0 & $\begin{array}{c}\text { UE A } \\
\text { initiates a } \\
\text { broadcast } \\
\text { group call }\end{array}$ & $\begin{array}{c}\text { B2: in } \\
\text { progress } \\
\text { broadcast } \\
\text { group call }\end{array}$ & - & - & $\begin{array}{l}\text { UE A generates } \\
\text { and stores SDP, } \\
\text { call identifier } \\
\text { and MCPTT } \\
\text { user ID of the } \\
\text { call, generates } \\
\text { and sends } \\
\text { GROUP CALL } \\
\text { BROADCAST, } \\
\text { starts floor } \\
\text { control as } \\
\text { originating floor } \\
\text { participant, } \\
\text { establishes } \\
\text { media session, } \\
\text { and starts TFB2 }\end{array}$ & 10.3.2.4.1 \\
\hline
\end{tabular}




\begin{tabular}{|c|c|c|c|c|c|c|c|}
\hline & Timeline & $\begin{array}{c}\text { Triggering } \\
\text { event }\end{array}$ & $\begin{array}{l}\text { UE A state } \\
\text { transition }\end{array}$ & $\begin{array}{l}\text { UE B state } \\
\text { transition }\end{array}$ & $\begin{array}{l}\text { UE C state } \\
\text { transition }\end{array}$ & $\begin{array}{c}\text { Consequent } \\
\text { behavior }\end{array}$ & $\begin{array}{c}\text { Reference } \\
\text { for } \\
\text { details }\end{array}$ \\
\hline 2 & $\begin{array}{l}\mathrm{T} 0+ \\
\mathrm{t} \_\mathrm{AB} 1\end{array}$ & $\begin{array}{l}\text { UE B and } \\
\text { UE C } \\
\text { receive } \\
\text { GROUP } \\
\text { CALL } \\
\text { BROADC } \\
\text { AST }\end{array}$ & - & $\begin{array}{l}\text { B3: pending } \\
\text { user action }\end{array}$ & $\begin{array}{c}\text { B2: in- } \\
\text { progress } \\
\text { broadcast } \\
\text { group call }\end{array}$ & $\begin{array}{l}\text { UE B stores the } \\
\text { call identifier, } \\
\text { current call type, } \\
\text { SDP, originating } \\
\text { MCPTT user ID } \\
\text { IE and MCPTT } \\
\text { group ID IE of } \\
\text { the call, and } \\
\text { starts TFB3; } \\
\text { UE C stores the } \\
\text { call identifier, } \\
\text { current call type, } \\
\text { SDP, originating } \\
\text { MCPTT user ID } \\
\text { IE and MCPTT } \\
\text { group ID IE of } \\
\text { the call, } \\
\text { establishes } \\
\text { media session, } \\
\text { starts floor } \\
\text { control as } \\
\text { terminating } \\
\text { participant and } \\
\text { starts TFB1 }\end{array}$ & 10.3.2.4.2 \\
\hline 3 & $\begin{array}{c}\mathrm{T} 0+ \\
\text { t_AB1 + } \\
\text { t_B1 }\end{array}$ & $\begin{array}{l}\text { UE B } \\
\text { accepts the } \\
\text { incoming } \\
\text { broadcast } \\
\text { group call }\end{array}$ & - & $\begin{array}{l}\text { B2: in- } \\
\text { progress } \\
\text { broadcast } \\
\text { group call }\end{array}$ & - & $\begin{array}{l}\text { UE B establishes } \\
\text { media session, } \\
\text { starts floor } \\
\text { control as } \\
\text { terminating } \\
\text { participant, stops } \\
\text { TFB3 and starts } \\
\text { TFB1 }\end{array}$ & 10.3.2.4.3 \\
\hline
\end{tabular}

\subsubsection{Call setup - establish a new call with refusal to join}

In this scenario, a user, UE A, decides to establish a broadcast group call (no existing one yet). UE B requires user acknowledgement, while UE C does not. One of the users, UE B, decides to reject the newly initiated call.

Scenario purpose: To observe that a new broadcast group call can be established and that a user can refuse to join the call.

Table 51: Call setup - establish a new call with refusal to join

\begin{tabular}{|c|c|c|c|c|c|c|c|}
\hline & Timeline & $\begin{array}{c}\text { Triggering } \\
\text { event }\end{array}$ & $\begin{array}{l}\text { UE A state } \\
\text { transition }\end{array}$ & $\begin{array}{l}\text { UE B state } \\
\text { transition }\end{array}$ & $\begin{array}{c}\text { UE C state } \\
\text { transition }\end{array}$ & $\begin{array}{c}\text { Consequent } \\
\text { behavior }\end{array}$ & $\begin{array}{l}\text { Reference } \\
\text { for details }\end{array}$ \\
\hline 0 & - & - & $\begin{array}{l}\text { B1: start- } \\
\text { stop }\end{array}$ & $\begin{array}{l}\text { B1: start- } \\
\text { stop }\end{array}$ & $\begin{array}{l}\text { B1: start- } \\
\text { stop }\end{array}$ & - & - \\
\hline
\end{tabular}




\begin{tabular}{|c|c|c|c|c|c|c|c|}
\hline & Timeline & $\begin{array}{c}\text { Triggering } \\
\text { event }\end{array}$ & $\begin{array}{l}\text { UE A state } \\
\text { transition }\end{array}$ & $\begin{array}{l}\text { UE B state } \\
\text { transition }\end{array}$ & $\begin{array}{l}\text { UE C state } \\
\text { transition }\end{array}$ & $\begin{array}{c}\text { Consequent } \\
\text { behavior }\end{array}$ & $\begin{array}{l}\text { Reference } \\
\text { for details }\end{array}$ \\
\hline 1 & T0 & $\begin{array}{c}\text { UE A initiates } \\
\text { a broadcast } \\
\text { group call }\end{array}$ & $\begin{array}{l}\text { B2: in } \\
\text { progress } \\
\text { broadcast } \\
\text { group call }\end{array}$ & - & - & $\begin{array}{l}\text { UE A generates } \\
\text { and stores SDP, } \\
\text { call identifier } \\
\text { and MCPTT } \\
\text { user ID of the } \\
\text { call, generates } \\
\text { and sends } \\
\text { GROUP CALL } \\
\text { BROADCAST, } \\
\text { starts floor } \\
\text { control as } \\
\text { originating floor } \\
\text { participant, } \\
\text { establishes } \\
\text { media session, } \\
\text { and starts TFB2 }\end{array}$ & 10.3.2.4.1 \\
\hline 2 & $\begin{array}{c}\mathrm{T} 0+ \\
\mathrm{t} \_\mathrm{AB} 1\end{array}$ & $\begin{array}{l}\text { UE } \mathbf{B} \text { and } \mathbf{U E} \\
\mathbf{C} \text { receive } \\
\text { GROUP } \\
\text { CALL } \\
\text { BROADCAST }\end{array}$ & - & $\begin{array}{c}\text { B3: } \\
\text { pending } \\
\text { user action }\end{array}$ & $\begin{array}{l}\text { B2: in- } \\
\text { progress } \\
\text { broadcast } \\
\text { group call }\end{array}$ & $\begin{array}{l}\text { UE B stores the } \\
\text { call identifier, } \\
\text { current call type, } \\
\text { SDP, originating } \\
\text { MCPTT user ID } \\
\text { IE and MCPTT } \\
\text { group ID IE of } \\
\text { the call, and } \\
\text { starts TFB3; } \\
\text { UE C stores the } \\
\text { call identifier, } \\
\text { current call type, } \\
\text { SDP, originating } \\
\text { MCPTT user ID } \\
\text { IE and MCPTT } \\
\text { group ID IE of } \\
\text { the call, } \\
\text { establishes } \\
\text { media session, } \\
\text { starts floor } \\
\text { control as } \\
\text { terminating } \\
\text { participant and } \\
\text { starts TFB1 }\end{array}$ & 10.3.2.4.2 \\
\hline 3 & $\begin{array}{c}\mathrm{T} 0+ \\
\mathrm{t} \_\mathrm{AB} 1+ \\
\mathrm{t} \_\mathrm{B} 1\end{array}$ & $\begin{array}{l}\text { UE B rejects } \\
\text { the incoming } \\
\text { broadcast } \\
\text { group call }\end{array}$ & - & $\begin{array}{c}\text { B4: } \\
\text { ignoring } \\
\text { same call } \\
\text { ID } \\
\end{array}$ & - & $\begin{array}{l}\text { UE B stops } \\
\text { TFB3 }\end{array}$ & 10.3.2.4.4 \\
\hline
\end{tabular}

And the corresponding figure is Fig. 14: 


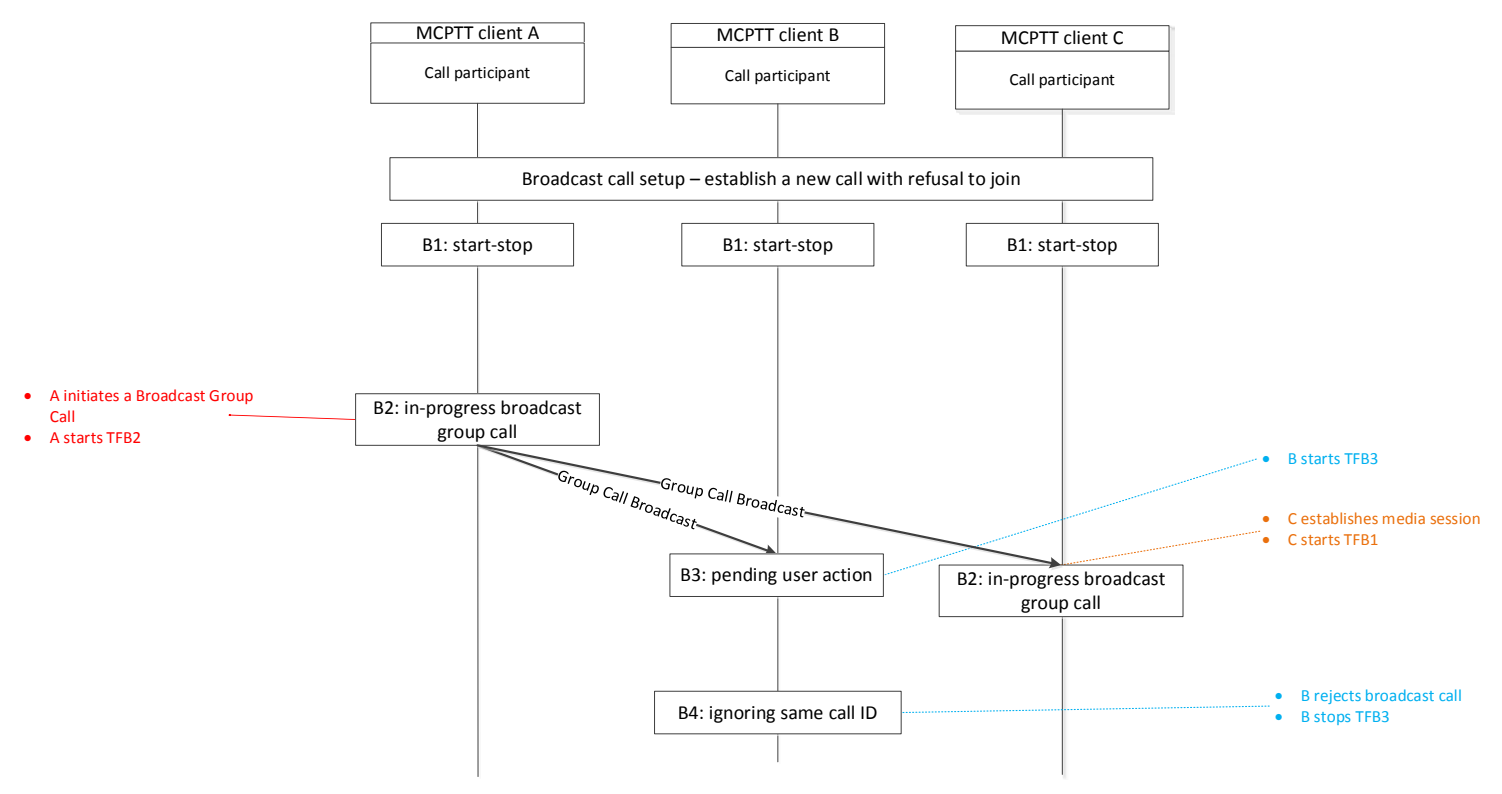

Figure 14: Broadcast call setup - establish a new call with refusal to join

\subsubsection{Call release}

\subsubsection{Call release by the originating user}

In this scenario, a broadcast group call is released by the originating user of the broadcast group call. UE A is considered the originating user. The originating user releases the call.

Scenario purpose: To observe that an ongoing broadcast group call can be released by the originating user.

Table 52: Call release by the originating user

\begin{tabular}{|c|c|c|c|c|c|c|c|}
\hline Timeline & $\begin{array}{c}\text { Triggering } \\
\text { event }\end{array}$ & $\begin{array}{c}\text { UE A state } \\
\text { transition }\end{array}$ & $\begin{array}{c}\text { UE B state } \\
\text { transition }\end{array}$ & $\begin{array}{c}\text { UE C state } \\
\text { transition }\end{array}$ & $\begin{array}{c}\text { Consequent } \\
\text { behavior }\end{array}$ & $\begin{array}{c}\text { Reference } \\
\text { for details }\end{array}$ \\
\hline 0 & - & - & $\begin{array}{c}\text { B2: in } \\
\text { progress } \\
\text { broadcast } \\
\text { group call }\end{array}$ & $\begin{array}{c}\text { B2: in } \\
\text { progress } \\
\text { broadcast } \\
\text { group call }\end{array}$ & $\begin{array}{c}\text { B2: in } \\
\text { progress } \\
\text { broadcast } \\
\text { group call }\end{array}$ & - & - \\
\hline
\end{tabular}




\begin{tabular}{|c|c|c|c|c|c|c|c|}
\hline & Timeline & $\begin{array}{c}\text { Triggering } \\
\text { event }\end{array}$ & $\begin{array}{l}\text { UE A state } \\
\text { transition }\end{array}$ & $\begin{array}{l}\text { UE B state } \\
\text { transition }\end{array}$ & $\begin{array}{l}\text { UE C state } \\
\text { transition }\end{array}$ & $\begin{array}{c}\text { Consequent } \\
\text { behavior }\end{array}$ & $\begin{array}{l}\text { Reference } \\
\text { for details }\end{array}$ \\
\hline 1 & T0 & $\begin{array}{c}\text { UE A } \\
\text { releases } \\
\text { broadcast } \\
\text { group call }\end{array}$ & $\begin{array}{l}\text { B1: start- } \\
\text { stop }\end{array}$ & - & - & $\begin{array}{c}\text { UE A releases } \\
\text { media session, } \\
\text { generates and } \\
\text { sends GROUP } \\
\text { CALL } \\
\text { BROADCAST } \\
\text { END, stops } \\
\text { floor control, } \\
\text { and stops TFB2 }\end{array}$ & 10.3 .2 .4 .7 \\
\hline 2 & $\begin{array}{l}\mathrm{T} 0+ \\
\mathrm{t} \_\mathrm{AB} 1\end{array}$ & $\begin{array}{l}\text { UE B and } \\
\text { UE C } \\
\text { receive } \\
\text { GROUP } \\
\text { CALL } \\
\text { BROADC } \\
\text { AST END }\end{array}$ & - & $\begin{array}{l}\text { B1: start- } \\
\text { stop }\end{array}$ & $\begin{array}{l}\text { B1: start- } \\
\text { stop }\end{array}$ & $\begin{array}{c}\mathbf{U E} \mathbf{B} \text { and } \mathbf{U E} \\
\mathbf{C} \text { release media } \\
\text { session and } \\
\text { stop floor } \\
\text { control }\end{array}$ & 10.3 .2 .4 .8 \\
\hline
\end{tabular}

\subsubsection{Call release by a participant}

In this scenario, a terminating user (i.e., not the originating user of the broadcast group call) releases from the broadcast group call. UE A is considered the originating user. A terminating user, UE B, releases the call. We consider this scenario starts after UE A has already talked for a while (nearing TFB1 expiry), and that there is only enough time left to retransmit the GROUP CALL BROADCAST message once.

Scenario purpose: To observe that an ongoing broadcast group call can be released by a participating user.

Table 53: Call release by a participant

\begin{tabular}{|c|c|c|c|c|c|c|c|}
\hline & Timeline & $\begin{array}{c}\text { Triggering } \\
\text { event }\end{array}$ & $\begin{array}{c}\text { UE A state } \\
\text { transition }\end{array}$ & $\begin{array}{c}\text { UE B state } \\
\text { transition }\end{array}$ & $\begin{array}{c}\text { UE C state } \\
\text { transition }\end{array}$ & $\begin{array}{c}\text { Consequent } \\
\text { behavior }\end{array}$ & $\begin{array}{c}\text { Reference } \\
\text { for details }\end{array}$ \\
\hline 0 & - & - & $\begin{array}{c}\text { B2: in } \\
\text { progress } \\
\text { broadcast } \\
\text { group call }\end{array}$ & $\begin{array}{c}\text { B2: in } \\
\text { progress } \\
\text { broadcast } \\
\text { group call }\end{array}$ & $\begin{array}{c}\text { B2: in } \\
\text { progress } \\
\text { broadcast } \\
\text { group call }\end{array}$ & - & - \\
\hline 1 & T0 & $\begin{array}{c}\text { UE B } \\
\text { progress } \\
\text { proadcast } \\
\text { group call }\end{array}$ & - & $\begin{array}{c}\text { B4: ignoring } \\
\text { same call ID }\end{array}$ & - & $\begin{array}{c}\text { UE B releases } \\
\text { the media } \\
\text { session, and } \\
\text { stops floor } \\
\text { control }\end{array}$ & $\mathbf{1 0 . 3 . 2 . 4 . 6}$ \\
\hline 2 & $\begin{array}{c}\text { T0 }+ \\
\text { UEA A }\end{array}$ & $\begin{array}{c}\text { TFB2 } \\
\text { TFpires }\end{array}$ & - & - & - & $\begin{array}{c}\text { UE A generates } \\
\text { and sends } \\
\text { GROUP CALL } \\
\text { BROADCAST, }\end{array}$ & $\mathbf{1 0 . 3 . 2 . 4 . 9}$ \\
and starts TFB2 & \\
\hline
\end{tabular}




\begin{tabular}{|c|c|c|c|c|c|c|c|}
\hline & Timeline & $\begin{array}{c}\text { Triggering } \\
\text { event }\end{array}$ & $\begin{array}{l}\text { UE A state } \\
\text { transition }\end{array}$ & $\begin{array}{l}\text { UE B state } \\
\text { transition }\end{array}$ & $\begin{array}{l}\text { UE C state } \\
\text { transition }\end{array}$ & $\begin{array}{c}\text { Consequent } \\
\text { behavior }\end{array}$ & $\begin{array}{l}\text { Reference } \\
\text { for details }\end{array}$ \\
\hline 3 & $\begin{array}{c}\text { T0 + } \\
\text { t_A1 }+ \\
\text { t_AB1 }\end{array}$ & $\begin{array}{l}\text { UE B and } \\
\text { UE C } \\
\text { receive } \\
\text { GROUP } \\
\text { CALL } \\
\text { BROADC } \\
\text { AST }\end{array}$ & - & - & - & $\begin{array}{c}\text { UE B checks } \\
\text { that identifier in } \\
\text { GROUP CALL } \\
\text { BROADCAST } \\
\text { matches with } \\
\text { the stored call } \\
\text { identifier, and } \\
\text { starts TFB1; } \\
\text { UE C: } \\
\text { undefined } \\
\text { action / } \\
\text { behavior } \\
\end{array}$ & $\begin{array}{r}\text { 10.3.2.4.10 } \\
\text { N/A }\end{array}$ \\
\hline 4 & $\begin{array}{c}\mathrm{T} 0+ \\
\mathrm{t} \_\mathrm{C} 1 \\
\text { (with } \\
t \_C 1> \\
\text { t_A1+ } \\
\left.t \_A B 1\right) \\
\end{array}$ & $\begin{array}{c}\text { UE C } \\
\text { TFB1 } \\
\text { expires }\end{array}$ & - & - & $\begin{array}{l}\text { B1: start- } \\
\text { stop }\end{array}$ & $\begin{array}{c}\text { UE C clears } \\
\text { stored call } \\
\text { identifier }\end{array}$ & 10.3.2.4.11 \\
\hline 5 & $\begin{array}{c}\text { T0 + } \\
\text { t_B1 } \\
\text { (with } \\
t \_B 1> \\
\text { t_A1+ } \\
\left.t \_A B 1\right)\end{array}$ & $\begin{array}{l}\text { UE B } \\
\text { TFB1 } \\
\text { expires }\end{array}$ & - & $\begin{array}{l}\text { B1: start- } \\
\text { stop }\end{array}$ & - & $\begin{array}{l}\text { UE B clears } \\
\text { stored call } \\
\text { identifier }\end{array}$ & 10.3.2.4.11 \\
\hline
\end{tabular}

And the corresponding figure is Fig. 15: 


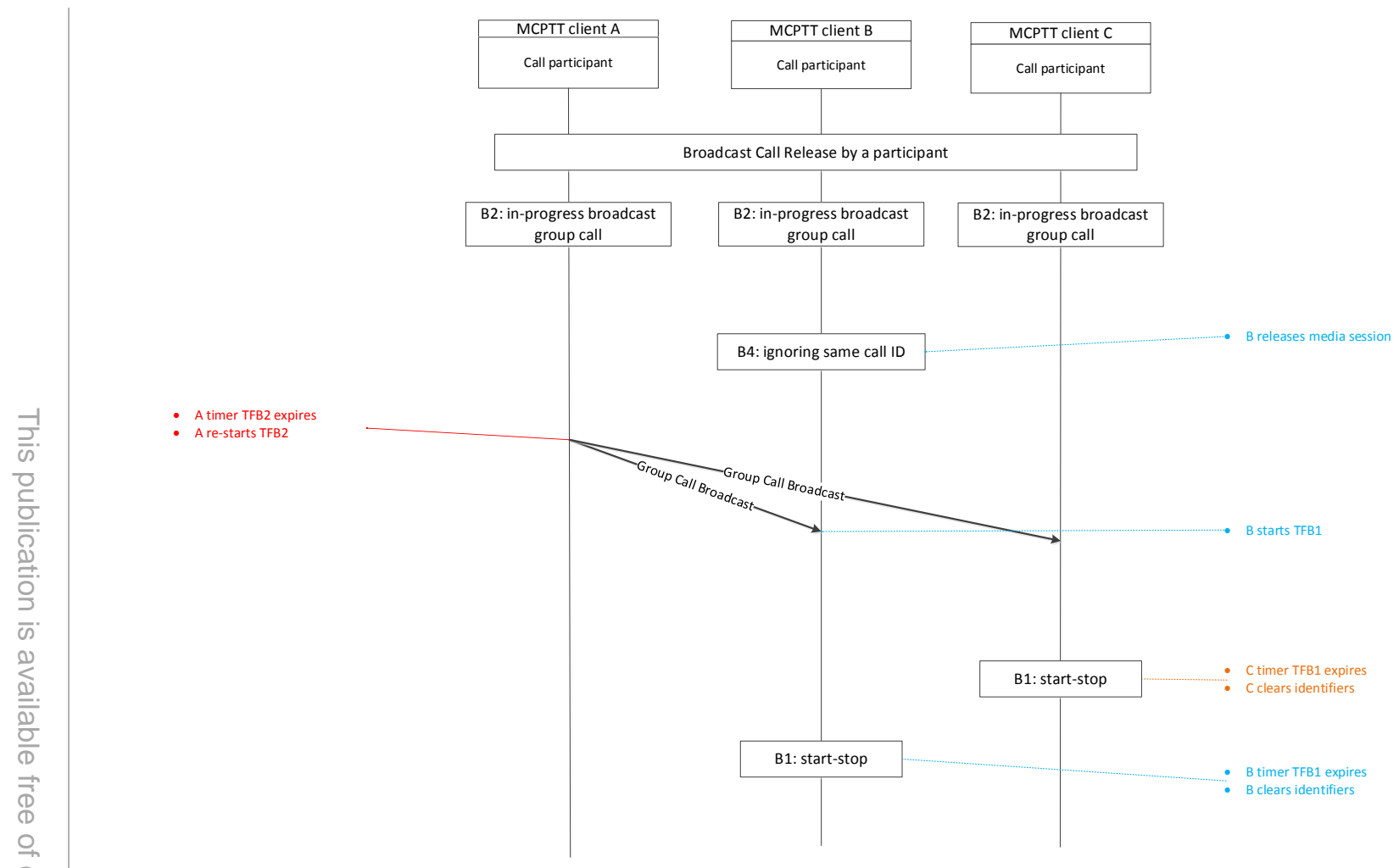

Figure 15: Broadcast call release by a participant 


\section{Test scenarios details - private call}

A private call is a call occurring between two MCPTT clients. A private call can operate under automatic commencement mode or the manual commencement mode, modifying the way the call shall be established. 3GPP TS 24.379 v14.2.0 [4], clause 11 describes the logic and functioning of private calls, specifically clause 11.2 for off-network private call.

\section{1. $\quad$ Private call - call control}

\subsubsection{Private call setup}

\subsubsection{Private call setup - establish new call in automatic mode}

In this scenario a user, UE A, decides to establish a private call (no existing one yet). The call is made using automatic commencement mode.

Scenario purpose: To observe that a private call can be initiated (in automatic commencement mode).

Table 54: Private call setup - establish new call in automatic mode

\begin{tabular}{|c|c|c|c|c|c|c|}
\hline & Timeline & $\begin{array}{c}\text { Triggering } \\
\text { event }\end{array}$ & $\begin{array}{l}\text { UE A state } \\
\text { transition }\end{array}$ & $\begin{array}{l}\text { UE B state } \\
\text { transition }\end{array}$ & Consequent behavior & $\begin{array}{l}\text { Reference } \\
\text { for details }\end{array}$ \\
\hline 0 & - & - & $\begin{array}{l}\text { P0: start- } \\
\text { stop }\end{array}$ & $\begin{array}{l}\text { P0: start- } \\
\text { stop }\end{array}$ & - & - \\
\hline 1 & T0 & $\begin{array}{l}\text { Indication } \\
\text { from UE A } \\
\text { to initiate a } \\
\text { private call } \\
\text { to UE B }\end{array}$ & $\begin{array}{l}\text { P2: waiting } \\
\text { for call } \\
\text { response }\end{array}$ & - & $\begin{array}{l}\text { UE A generates and stores Call } \\
\text { Identifier, MCPTT user ID, user } \\
\text { ID of the callee, user location, } \\
\text { offer SDP, creates call type } \\
\text { control state machine and } \\
\text { establishes end-to-end security } \\
\text { (if needed), stores } \\
\text { commencement mode as } \\
\text { "AUTOMATIC } \\
\text { COMMENCEMENT MODE", } \\
\text { generates and sends PRIVATE } \\
\text { CALL SETUP REQUEST, } \\
\text { starts TFP1 and initializes CFP1 }\end{array}$ & 11.2.2.4.2.1 \\
\hline 2 & $\begin{array}{l}\mathrm{T} 0+ \\
\text { t_AB1 }\end{array}$ & $\begin{array}{c}\text { UE B } \\
\text { receives } \\
\text { PRIVATE } \\
\text { CALL } \\
\text { SETUP } \\
\text { REQUEST }\end{array}$ & - & P5: pending & $\begin{array}{l}\text { UE B stores Call Identifier IE } \\
\text { as call identifier, MCPTT user } \\
\text { ID of the caller IE as caller ID } \\
\text { and own MCPTT user ID as } \\
\text { callee IE, creates call type } \\
\text { control state machine, generates } \\
\text { and stores answer SDP, } \\
\text { generates and sends PRIVATE } \\
\text { CALL ACCEPT, establishes a } \\
\text { media session, starts TFP4 and } \\
\text { initializes CFP4 }\end{array}$ & 11.2.2.4.3.2 \\
\hline
\end{tabular}




\begin{tabular}{|c|c|c|c|c|c|c|}
\hline & Timeline & $\begin{array}{c}\text { Triggering } \\
\text { event }\end{array}$ & $\begin{array}{l}\text { UE A state } \\
\text { transition }\end{array}$ & $\begin{array}{l}\text { UE B state } \\
\text { transition }\end{array}$ & Consequent behavior & $\begin{array}{l}\text { Reference } \\
\text { for details }\end{array}$ \\
\hline 3 & $\begin{array}{c}\text { T0 + } \\
\text { t_AB1 + } \\
\text { t_BA1 }\end{array}$ & $\begin{array}{c}\text { UE A } \\
\text { receives } \\
\text { PRIVATE } \\
\text { CALL } \\
\text { ACCEPT }\end{array}$ & $\begin{array}{l}\text { P4: part of } \\
\text { ongoing } \\
\text { call }\end{array}$ & - & $\begin{array}{c}\text { UE A stores SDP answer, } \\
\text { generates and sends PRIVATE } \\
\text { CALL ACCEPT ACK, } \\
\text { establishes a media session, } \\
\text { starts floor control as } \\
\text { terminating floor participant, } \\
\text { stops TFP1, and starts TFP5 }\end{array}$ & 11.2.2.4.2.8 \\
\hline 4 & $\begin{array}{c}\text { T0 + } \\
\text { t_AB1 + } \\
\text { t_BA1 + } \\
\text { t_AB2 }\end{array}$ & $\begin{array}{c}\text { UE B } \\
\text { receives } \\
\text { PRIVATE } \\
\text { CALL } \\
\text { ACCEPT } \\
\text { ACK }\end{array}$ & - & $\begin{array}{l}\text { P4: part of } \\
\text { ongoing call }\end{array}$ & $\begin{array}{l}\text { UE B starts floor control as } \\
\text { terminating MCPTT client, } \\
\text { stops TFP4 and starts TFP5 }\end{array}$ & 11.2.2.4.3.4 \\
\hline
\end{tabular}

And the corresponding figure is Fig. 16:

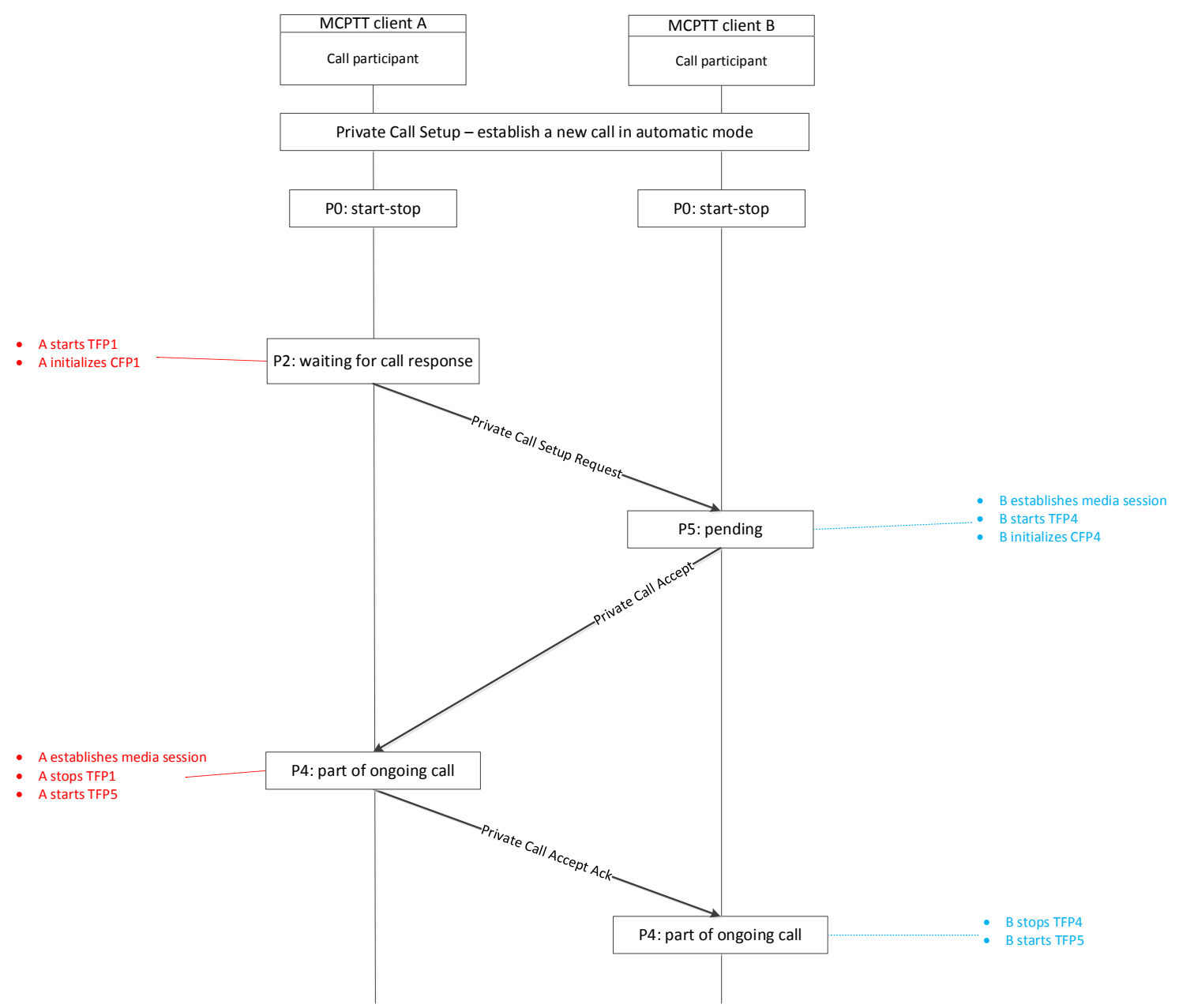

Figure 16: Private call setup - establish new call in automatic mode 


\subsubsection{Private call setup - establish and cancel new call in automatic mode}

In this scenario a user, UE A, decides to establish a private call (no existing one yet). The call is made using automatic commencement mode. The UE initiating the call decides to cancel the call almost immediately afterwards and the SDP offer does not contain a dedicated key attribute.

Scenario purpose: To observe that a private call can be initiated (in automatic commencement mode) and canceled before it is established.

Table 55: Private call setup - establish and cancel new call in automatic mode

\begin{tabular}{|c|c|c|c|c|c|c|}
\hline & Timeline & $\begin{array}{c}\text { Triggering } \\
\text { event }\end{array}$ & $\begin{array}{l}\text { UE A state } \\
\text { transition }\end{array}$ & $\begin{array}{l}\text { UE B state } \\
\text { transition }\end{array}$ & Consequent behavior & $\begin{array}{l}\text { Reference } \\
\text { for details }\end{array}$ \\
\hline 0 & - & - & P0: start-stop & P0: start-stop & -- & - \\
\hline 1 & T0 & $\begin{array}{l}\text { Indication } \\
\text { from UE A to } \\
\text { initiate a } \\
\text { private call to } \\
\text { UE B }\end{array}$ & $\begin{array}{l}\text { P2: waiting } \\
\text { for call } \\
\text { response }\end{array}$ & - & $\begin{array}{l}\text { UE A generates and } \\
\text { stores Call Identifier, } \\
\text { MCPTT user ID, user } \\
\text { ID of the callee, user } \\
\text { location, offer SDP, } \\
\text { creates call type control } \\
\text { state machine and } \\
\text { establishes end-to-end } \\
\text { security (if needed), } \\
\text { stores commencement } \\
\text { mode as } \\
\text { "AUTOMATIC } \\
\text { COMMENCEMENT } \\
\text { MODE", generates and } \\
\text { sends PRIVATE CALL } \\
\text { SETUP REQUEST, } \\
\text { starts TFP1 and } \\
\text { initializes CFP1 }\end{array}$ & 11.2.2.4.2.1 \\
\hline 2 & $\begin{array}{l}\text { T0 + } \\
\text { t_AB1 }\end{array}$ & $\begin{array}{c}\text { UE B } \\
\text { receives } \\
\text { PRIVATE } \\
\text { CALL } \\
\text { SETUP } \\
\text { REQUEST }\end{array}$ & - & P5: pending & $\begin{array}{c}\text { UE B stores Call } \\
\text { Identifier IE as call } \\
\text { identifier, MCPTT user } \\
\text { ID of the caller IE as } \\
\text { caller ID and own } \\
\text { MCPTT user ID as } \\
\text { callee IE, creates call } \\
\text { type control state } \\
\text { machine, generates and } \\
\text { stores answer SDP, } \\
\text { generates and sends } \\
\text { PRIVATE CALL } \\
\text { ACCEPT, establishes a } \\
\text { media session, starts } \\
\text { TFP4 and initializes } \\
\text { CFP4 }\end{array}$ & 11.2.2.4.3.2 \\
\hline
\end{tabular}




\begin{tabular}{|c|c|c|c|c|c|c|}
\hline & Timeline & $\begin{array}{l}\text { Triggering } \\
\text { event }\end{array}$ & $\begin{array}{l}\text { UE A state } \\
\text { transition }\end{array}$ & $\begin{array}{l}\text { UE B state } \\
\text { transition }\end{array}$ & Consequent behavior & $\begin{array}{l}\text { Reference } \\
\text { for details }\end{array}$ \\
\hline 3 & $\begin{array}{l}\text { T0 + } \\
\text { t_A1 }\end{array}$ & $\begin{array}{l}\text { UE A cancels } \\
\text { the private } \\
\text { call request }\end{array}$ & $\begin{array}{l}\text { P3: waiting } \\
\text { for release } \\
\text { response }\end{array}$ & - & $\begin{array}{c}\text { UE A generates and } \\
\text { sends PRIVATE CALL } \\
\text { RELEASE, and starts } \\
\text { TFP3 }\end{array}$ & 11.2.2.4.2.9 \\
\hline 4 & $\begin{array}{c}\mathrm{T} 0+ \\
\text { t_AB1 + } \\
\text { t_BA1 }\end{array}$ & $\begin{array}{c}\text { UE A } \\
\text { receives } \\
\text { PRIVATE } \\
\text { CALL } \\
\text { ACCEPT }\end{array}$ & - & - & $\begin{array}{l}\text { UE A discards the } \\
\text { message }\end{array}$ & 11.2.2.4.6.1 \\
\hline 5 & $\begin{array}{l}\mathrm{T} 0+ \\
\mathrm{t} \_\mathrm{A} 1+ \\
\mathrm{t} \_\mathrm{AB} 2\end{array}$ & $\begin{array}{c}\mathbf{U E ~ B} \\
\text { receives } \\
\text { PRIVATE } \\
\text { CALL } \\
\text { RELEASE } \\
\end{array}$ & - & - & $\begin{array}{l}\text { UE B discards the } \\
\text { message }\end{array}$ & 11.2.2.4.6.1 \\
\hline 6 & $\begin{array}{c}\mathrm{T} 0+ \\
\mathrm{t} \_\mathrm{A} 1+ \\
\mathrm{n}^{*} \mathrm{TFP} 3 \\
\text { (with } \\
\text { CFP3 at } \\
\text { its upper } \\
\text { limit) }\end{array}$ & $\begin{array}{l}\text { UE A TFP3 } \\
\text { expires }\end{array}$ & $\begin{array}{l}\text { P1: ignoring } \\
\text { same call ID }\end{array}$ & & $\begin{array}{c}\text { UE A terminates the } \\
\text { media session, releases } \\
\text { the call type control } \\
\text { state machine, and } \\
\text { starts TFP7 }\end{array}$ & 11.2.2.4.5.3 \\
\hline 7 & $\begin{array}{c}\text { T0+ } \\
\mathrm{t} \_\mathrm{A} 1+ \\
\mathrm{n}^{*} \mathrm{TFP} 3 \\
+ \text { TFP7 }\end{array}$ & $\begin{array}{l}\text { UE A TFP7 } \\
\text { expires }\end{array}$ & P0: start-stop & & $\begin{array}{l}\text { UE A clears the stored } \\
\text { call identifier }\end{array}$ & 11.2.2.4.5.7 \\
\hline
\end{tabular}

And the corresponding figure is Fig. 17: 


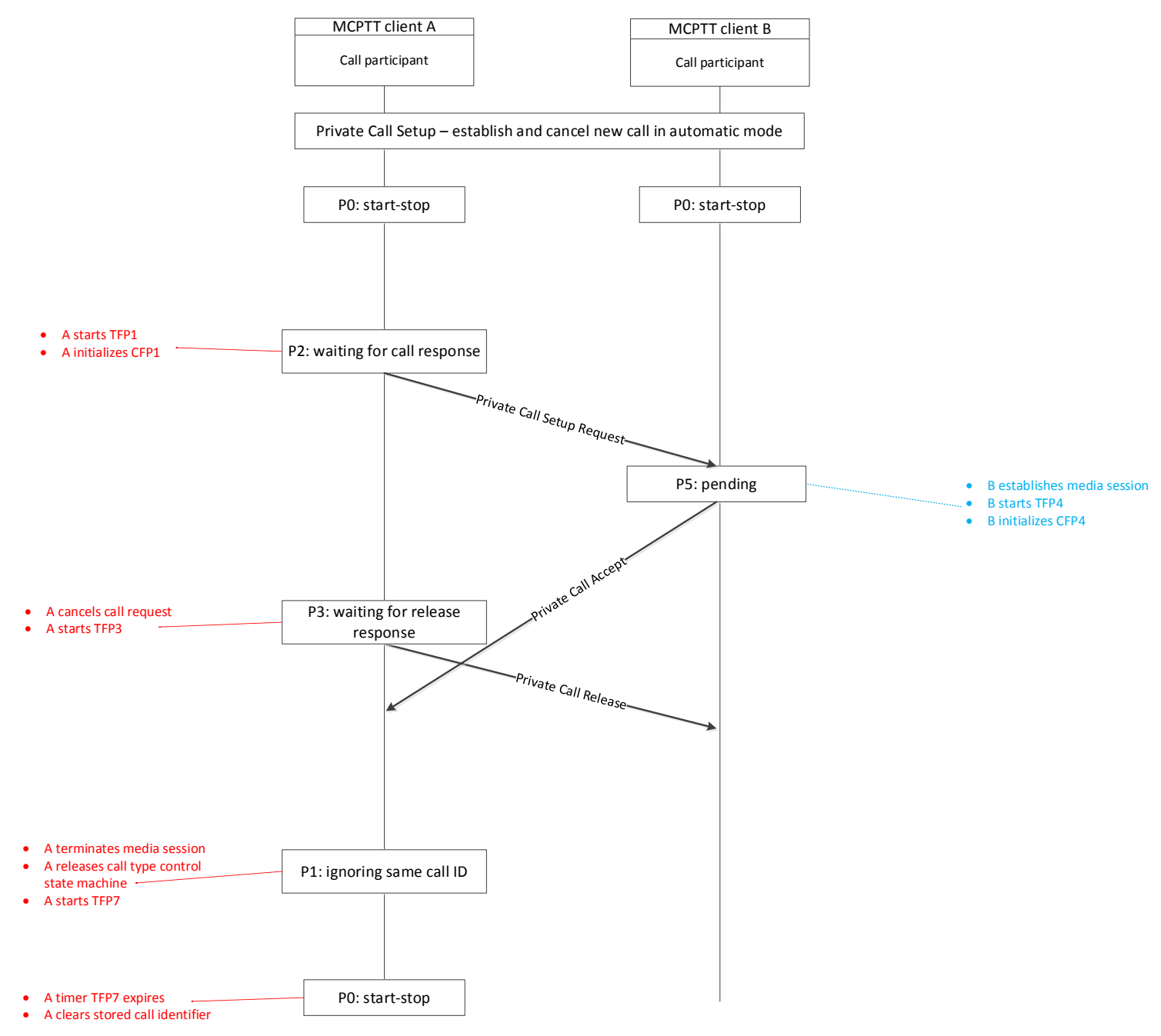

Figure 17: Private call setup - establish and cancel new call in automatic mode

\subsubsection{Private call setup - establish new call in manual mode}

In this scenario a user, UE A, decides to establish a private call (no existing one yet). The call is made using manual commencement mode, and the SDP offer does not contain a dedicated key attribute.

Scenario purpose: To observe that a private call can be initiated (in manual commencement mode), without any key attribute in the SDP offer. 
Table 56: Private call setup - establish new call in manual mode

\begin{tabular}{|c|c|c|c|c|c|c|}
\hline & Timeline & $\begin{array}{c}\text { Triggering } \\
\text { event }\end{array}$ & $\begin{array}{l}\text { UE A state } \\
\text { transition }\end{array}$ & $\begin{array}{l}\text { UE B state } \\
\text { transition }\end{array}$ & Consequent behavior & $\begin{array}{l}\text { Reference } \\
\text { for details }\end{array}$ \\
\hline 0 & - & - & P0: start-stop & P0: start-stop & - & - \\
\hline 1 & T0 & $\begin{array}{l}\text { Indication } \\
\text { from UE A } \\
\text { to initiate a } \\
\text { private call } \\
\text { to UE B }\end{array}$ & $\begin{array}{l}\text { P2: waiting } \\
\text { for call } \\
\text { response }\end{array}$ & - & $\begin{array}{l}\text { UE A generates and stores } \\
\text { Call Identifier, MCPTT } \\
\text { user ID, user ID of the } \\
\text { callee, user location, offer } \\
\text { SDP, creates call type } \\
\text { control state machine, } \\
\text { stores commencement } \\
\text { mode as "MANUAL } \\
\text { COMMENCEMENT } \\
\text { MODE", generates and } \\
\text { sends PRIVATE CALL } \\
\text { SETUP REQUEST, starts } \\
\text { TFP1 and initializes CFP1 }\end{array}$ & 11.2.2.4.2.1 \\
\hline 2 & $\begin{array}{l}\mathrm{T} 0+ \\
\mathrm{t} \_\mathrm{AB} 1\end{array}$ & $\begin{array}{c}\text { UE B } \\
\text { receives } \\
\text { PRIVATE } \\
\text { CALL } \\
\text { SETUP } \\
\text { REQUEST }\end{array}$ & - & P5: pending & $\begin{array}{l}\text { UE B stores Call Identifier } \\
\text { IE as call identifier, } \\
\text { MCPTT user ID of the } \\
\text { caller IE as caller ID and } \\
\text { own MCPTT user ID as } \\
\text { callee ID, creates call type } \\
\text { control state machine, } \\
\text { generates and stores } \\
\text { answer SDP, generates and } \\
\text { sends PRIVATE CALL } \\
\text { RINGING, and starts TFP2 }\end{array}$ & 11.2.2.4.4.1 \\
\hline 3 & $\begin{array}{c}\mathrm{T} 0+ \\
\mathrm{t} \_\mathrm{AB} 1+ \\
\mathrm{t} \_\mathrm{BA} 1\end{array}$ & $\begin{array}{c}\text { UE A } \\
\text { receives } \\
\text { PRIVATE } \\
\text { CALL } \\
\text { RINGING } \\
\end{array}$ & - & - & UE A remains in its state & 11.2.2.4.2.3 \\
\hline 4 & $\begin{array}{c}\text { T0 + t_B1 } \\
\text { (with } \\
t \_B 1> \\
\left.t \_A B 1\right)\end{array}$ & $\begin{array}{c}\text { UE B } \\
\text { accepts the } \\
\text { incoming } \\
\text { private call }\end{array}$ & - & - & $\begin{array}{l}\text { UE B generates and stores } \\
\text { SDP, generates and sends } \\
\text { PRIVATE CALL } \\
\text { ACCEPT, establishes a } \\
\text { media session, stops TFP2, } \\
\text { starts TFP4 and initializes } \\
\text { CFP4 }\end{array}$ & 11.2.2.4.4.3 \\
\hline 5 & $\begin{array}{l}\text { T0 + t_B1 } \\
+\mathrm{t} \_\mathrm{BA} 2\end{array}$ & $\begin{array}{c}\text { UE A } \\
\text { receives } \\
\text { PRIVATE } \\
\text { CALL } \\
\text { ACCEPT }\end{array}$ & $\begin{array}{l}\text { P4: part of } \\
\text { ongoing call }\end{array}$ & - & $\begin{array}{l}\text { UE A stores SDP answer, } \\
\text { generates and sends } \\
\text { PRIVATE CALL } \\
\text { ACCEPT ACK, establishes } \\
\text { a media session, starts } \\
\text { floor control as terminating } \\
\text { floor participant, stops } \\
\text { TFP1 and TFP2, and starts } \\
\text { TFP5 }\end{array}$ & 11.2.2.4.2.8 \\
\hline
\end{tabular}




\begin{tabular}{|c|c|c|c|c|c|c|}
\hline & Timeline & $\begin{array}{c}\text { Triggering } \\
\text { event }\end{array}$ & $\begin{array}{l}\text { UE A state } \\
\text { transition }\end{array}$ & $\begin{array}{l}\text { UE B state } \\
\text { transition }\end{array}$ & Consequent behavior & $\begin{array}{l}\text { Reference } \\
\text { for details }\end{array}$ \\
\hline 6 & $\begin{array}{l}\text { T0 + t_B1 } \\
+\mathrm{t} \_\mathrm{BA} 2 \\
+\mathrm{t} \_\mathrm{AB} 2\end{array}$ & $\begin{array}{c}\text { UE B } \\
\text { receives } \\
\text { PRIVATE } \\
\text { CALL } \\
\text { ACCEPT } \\
\text { ACK } \\
\end{array}$ & - & $\begin{array}{l}\text { P4: part of } \\
\text { ongoing call }\end{array}$ & $\begin{array}{c}\text { UE B starts floor control as } \\
\text { terminating MCPTT client, } \\
\text { stops TFP4 and starts } \\
\text { TFP5 }\end{array}$ & 11.2.2.4.4.5 \\
\hline
\end{tabular}

And the corresponding figure is Fig. 18:

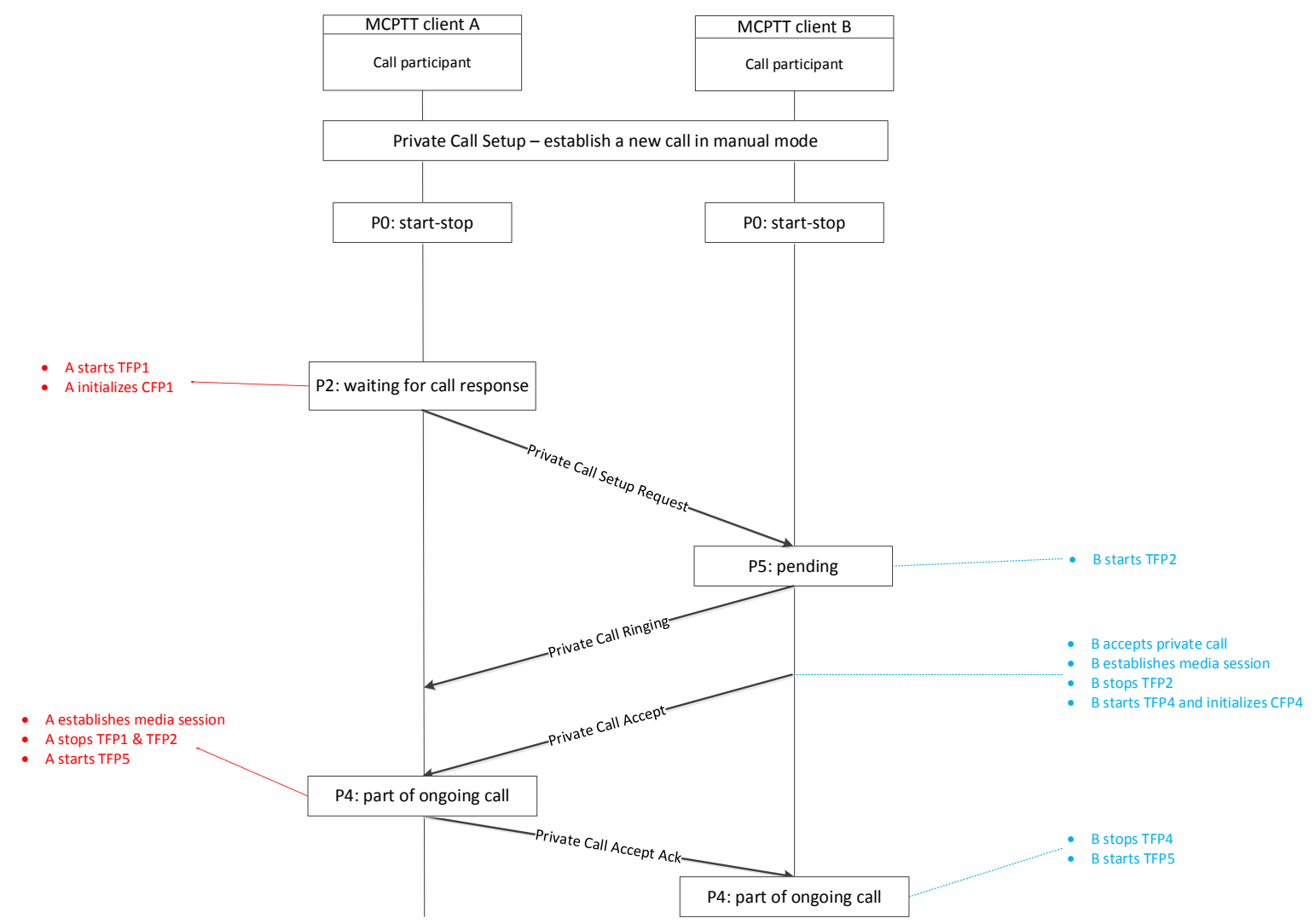

Figure 18: Private call setup - establish new call in manual mode

\subsubsection{Private call setup - establish and cancel new call in manual mode}

In this scenario a user, UE A, decides to establish a private call (no existing one yet). The call is made using manual commencement mode. The UE initiating the call decides to cancel it almost immediately after.

Scenario purpose: To observe that a private call can be initiated (in manual commencement mode) and canceled before it is established. 
Table 57: Private call setup - establish and cancel new call in manual mode

\begin{tabular}{|c|c|c|c|c|c|c|}
\hline & Timeline & $\begin{array}{c}\text { Triggering } \\
\text { event }\end{array}$ & $\begin{array}{c}\text { UE A } \\
\text { state } \\
\text { transition } \\
\end{array}$ & $\begin{array}{c}\text { UE B } \\
\text { state } \\
\text { transition } \\
\end{array}$ & Consequent behavior & $\begin{array}{l}\text { Reference } \\
\text { for details }\end{array}$ \\
\hline 0 & - & - & $\begin{array}{l}\text { P0: start- } \\
\text { stop }\end{array}$ & $\begin{array}{l}\text { P0: start- } \\
\text { stop }\end{array}$ & - & - \\
\hline 1 & T0 & $\begin{array}{l}\text { Indication } \\
\text { from UE A } \\
\text { to initiate a } \\
\text { private call } \\
\text { to UE B }\end{array}$ & $\begin{array}{l}\text { P2: } \\
\text { waiting } \\
\text { for call } \\
\text { response }\end{array}$ & - & $\begin{array}{l}\text { UE A generates and stores Call } \\
\text { Identifier, MCPTT user ID, user } \\
\text { ID of the callee, user location, } \\
\text { offer SDP, creates call type } \\
\text { control state machine, stores } \\
\text { commencement mode as } \\
\text { "MANUAL } \\
\text { COMMENCEMENT MODE", } \\
\text { generates and sends PRIVATE } \\
\text { CALL SETUP REQUEST, starts } \\
\text { TFP1 and initializes CFP1 }\end{array}$ & 11.2.2.4.2.1 \\
\hline 2 & $\begin{array}{l}\mathrm{T} 0+ \\
\mathrm{t} \_\mathrm{AB} 1\end{array}$ & $\begin{array}{l}\text { UE B } \\
\text { receives } \\
\text { PRIVATE } \\
\text { CALL } \\
\text { SETUP } \\
\text { REQUEST }\end{array}$ & - & $\begin{array}{c}\text { P5: } \\
\text { pending }\end{array}$ & $\begin{array}{l}\text { UE B stores Call Identifier IE as } \\
\text { call identifier, MCPTT user ID } \\
\text { of the caller IE as caller ID and } \\
\text { own MCPTT user ID as callee } \\
\text { ID, creates call type control state } \\
\text { machine, generates and stores } \\
\text { answer SDP, generates and sends } \\
\text { PRIVATE CALL RINGING, } \\
\text { and starts TFP2 }\end{array}$ & 11.2.2.4.4.1 \\
\hline 3 & $\begin{array}{l}\text { T0 + } \\
\text { t_A1 }\end{array}$ & $\begin{array}{c}\text { UE A } \\
\text { cancels the } \\
\text { private call } \\
\text { request }\end{array}$ & $\begin{array}{c}\text { P3: } \\
\text { waiting } \\
\text { for release } \\
\text { response }\end{array}$ & - & $\begin{array}{c}\text { UE A generates and sends } \\
\text { PRIVATE CALL RELEASE, } \\
\text { and starts TFP3 }\end{array}$ & 11.2.2.4.2.9 \\
\hline 4 & $\begin{array}{c}\text { T0 + } \\
\text { t_AB1 + } \\
\text { t_BA1 }\end{array}$ & $\begin{array}{c}\text { UE A } \\
\text { receives } \\
\text { PRIVATE } \\
\text { CALL } \\
\text { RINGING }\end{array}$ & - & - & $\begin{array}{l}\text { UE A discards the call control } \\
\text { message }\end{array}$ & 11.2.2.4.6.1 \\
\hline 5 & $\begin{array}{l}\text { T0+ } \\
\text { t_A1 }+ \\
\text { t_AB2 }\end{array}$ & $\begin{array}{l}\text { UE B } \\
\text { receives } \\
\text { PRIVATE } \\
\text { CALL } \\
\text { RELEASE }\end{array}$ & - & $\begin{array}{l}\text { P1: } \\
\text { ignoring } \\
\text { same call } \\
\text { ID }\end{array}$ & $\begin{array}{l}\text { UE B generates and sends } \\
\text { PRIVATE CALL RELEASE } \\
\text { ACK, stops TFP4, starts TFP7 } \\
\text { and releases the call type control } \\
\text { state machine }\end{array}$ & 11.2.2.4.4.8 \\
\hline 6 & $\begin{array}{l}\text { T0+ } \\
\text { t_A1 + } \\
\text { t_AB2 + } \\
\text { t_BA2 }\end{array}$ & $\begin{array}{c}\text { UE A } \\
\text { receives } \\
\text { PRIVATE } \\
\text { CALL } \\
\text { RELEASE } \\
\text { ACK } \\
\end{array}$ & $\begin{array}{l}\text { P1: } \\
\text { ignoring } \\
\text { same call } \\
\text { ID }\end{array}$ & - & $\begin{array}{c}\text { UE A terminates the media } \\
\text { session, stops TFP3 and starts } \\
\text { TFP7 }\end{array}$ & 11.2.2.4.5.5 \\
\hline
\end{tabular}

And the corresponding figure is Fig. 19: 


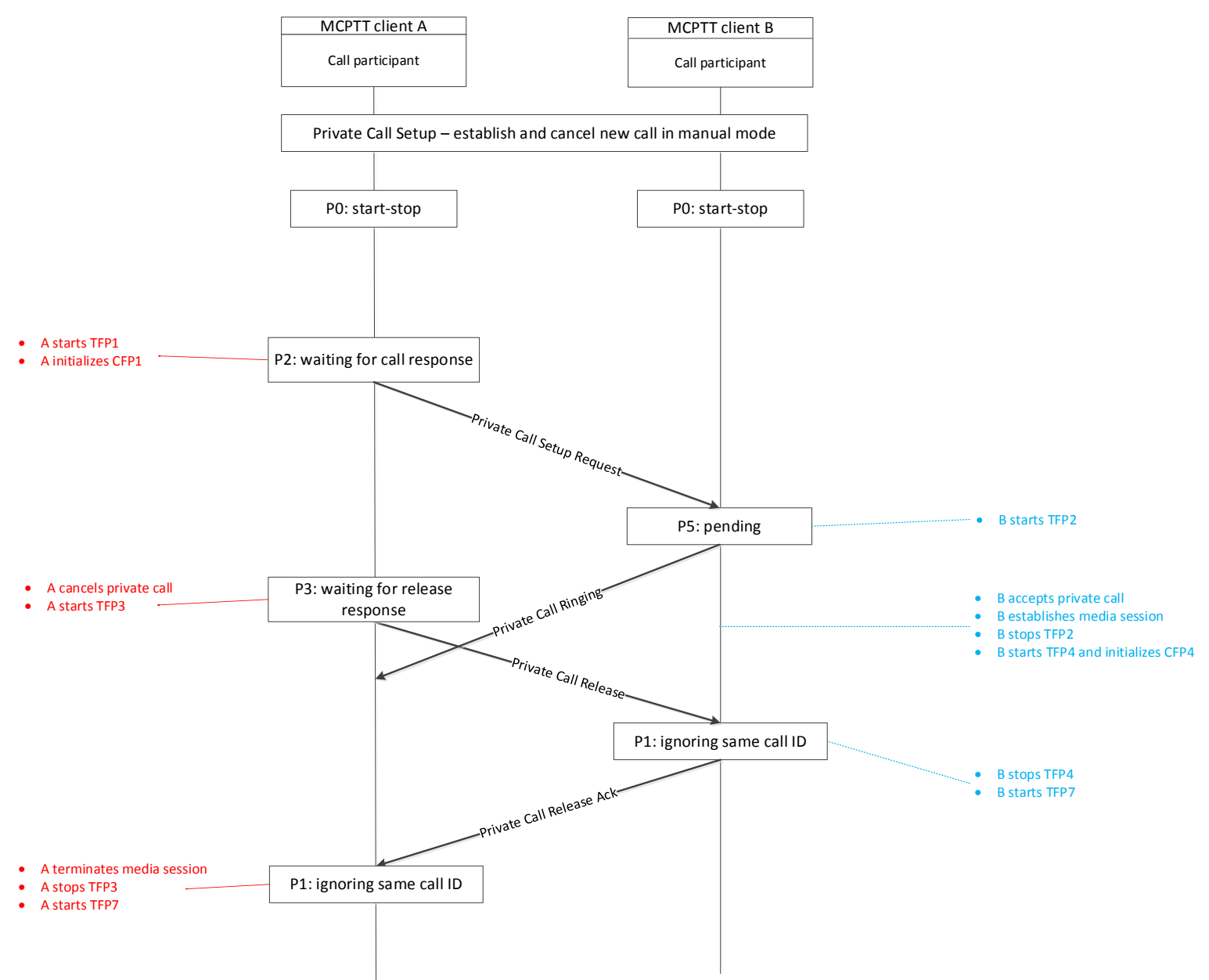

Figure 19: Private call setup - establish and cancel new call in manual mode

\subsubsection{Private call setup - failure to establish new call}

In this scenario a user, UE A, decides to establish a private call. The call is made using automatic commencement mode. Both users have different call identifiers, hence the call fails to establish. Two attempts are made by UE A.

Scenario purpose: To observe that a private call cannot be established between two users with different call identifiers.

Table 58: Private call setup - failure to establish a new call

\begin{tabular}{|c|c|c|c|c|c|c|}
\hline Timeline & $\begin{array}{c}\text { Triggering } \\
\text { event }\end{array}$ & $\begin{array}{c}\text { UE A state } \\
\text { transition }\end{array}$ & $\begin{array}{c}\text { UE B state } \\
\text { transition }\end{array}$ & Consequent behavior & $\begin{array}{c}\text { Reference } \\
\text { for details }\end{array}$ \\
\hline 0 & - & - & P0: start-stop & P0: start-stop & & - \\
\hline
\end{tabular}




\begin{tabular}{|c|c|c|c|c|c|c|}
\hline & Timeline & $\begin{array}{l}\text { Triggering } \\
\text { event }\end{array}$ & $\begin{array}{l}\text { UE A state } \\
\text { transition }\end{array}$ & $\begin{array}{l}\text { UE B state } \\
\text { transition }\end{array}$ & Consequent behavior & $\begin{array}{l}\text { Reference } \\
\text { for details }\end{array}$ \\
\hline 1 & T0 & $\begin{array}{c}\text { Indication } \\
\text { from UE A to } \\
\text { initiate a } \\
\text { private call to } \\
\text { UE B }\end{array}$ & $\begin{array}{l}\text { P2: waiting for call } \\
\text { response }\end{array}$ & - & $\begin{array}{l}\text { UE A generates and stores } \\
\text { Call Identifier, MCPTT user } \\
\text { ID, user ID of the callee, } \\
\text { user location, offer SDP, } \\
\text { creates call type control state } \\
\text { machine and establishes end- } \\
\text { to-end security (if needed), } \\
\text { stores commencement mode } \\
\text { as "AUTOMATIC } \\
\text { COMMENCEMENT } \\
\text { MODE", generates and } \\
\text { sends PRIVATE CALL } \\
\text { SETUP REQUEST, starts } \\
\text { TFP1 and initializes CFP1 } \\
\end{array}$ & 11.2.2.4.2.1 \\
\hline 2 & $\begin{array}{l}\mathrm{T} 0+ \\
\mathrm{t} \_\mathrm{AB} 1\end{array}$ & $\begin{array}{l}\text { UE B } \\
\text { receives } \\
\text { PRIVATE } \\
\text { CALL } \\
\text { SETUP } \\
\text { REQUEST }\end{array}$ & - & $\begin{array}{l}\text { P1: ignoring } \\
\text { same call ID }\end{array}$ & $\begin{array}{c}\text { UE B compares and } \\
\text { acknowledges difference } \\
\text { between Call Identifiers, } \\
\text { stores Call Identifier IE of } \\
\text { received message as call } \\
\text { identifier, MCPTT user ID } \\
\text { of caller IE as caller ID, own } \\
\text { MCPTT user ID as callee ID, } \\
\text { generates and sends } \\
\text { PRIVATE CALL REJECT, } \\
\text { and starts TFP7 }\end{array}$ & 11.2.2.4.3.1 \\
\hline 3 & $\begin{array}{c}\mathrm{T} 0+ \\
\mathrm{t} \_\mathrm{AB} 1+ \\
\mathrm{t} \_\mathrm{BA} 1\end{array}$ & $\begin{array}{l}\text { UE A } \\
\text { receives } \\
\text { PRIVATE } \\
\text { CALL } \\
\text { REJECT }\end{array}$ & $\begin{array}{l}\text { P1: ignoring same } \\
\text { call ID }\end{array}$ & - & $\begin{array}{l}\text { UE A releases call control } \\
\text { state machine, stops TFP1 } \\
\text { and TFP2, and starts TFP7 }\end{array}$ & 11.2.2.4.2.7 \\
\hline 4 & $\begin{array}{c}\text { T0 + } \\
\text { t_AB1 + } \\
\text { t_BA1 + } \\
\text { t_A1 }\end{array}$ & $\begin{array}{l}\text { Indication } \\
\text { from UE A to } \\
\text { initiate a } \\
\text { private call }\end{array}$ & $\begin{array}{l}\text { P2: waiting for call } \\
\text { response }\end{array}$ & - & $\begin{array}{l}\text { UE A generates and stores } \\
\text { Call Identifier, MCPTT user } \\
\text { ID, user ID of the callee, } \\
\text { user location, offer SDP, } \\
\text { creates call type control state } \\
\text { machine and establishes end- } \\
\text { to-end security (if needed), } \\
\text { stores commencement mode } \\
\text { as "AUTOMATIC } \\
\text { COMMENCEMENT } \\
\text { MODE", generates and } \\
\text { sends PRIVATE CALL } \\
\text { SETUP REQUEST, starts } \\
\text { TFP1 and initializes CFP1 }\end{array}$ & 11.2.2.4.2.1 \\
\hline
\end{tabular}




\begin{tabular}{|c|c|c|c|c|c|c|}
\hline & Timeline & $\begin{array}{c}\text { Triggering } \\
\text { event }\end{array}$ & $\begin{array}{l}\text { UE A state } \\
\text { transition }\end{array}$ & $\begin{array}{l}\text { UE B state } \\
\text { transition }\end{array}$ & Consequent behavior & $\begin{array}{l}\text { Reference } \\
\text { for details }\end{array}$ \\
\hline 5 & $\begin{array}{c}\text { T0 + } \\
\text { t_AB1 + } \\
\text { t_BA1 + } \\
\text { t_A1 + } \\
\text { t_AB2 }\end{array}$ & $\begin{array}{c}\text { UE B } \\
\text { receives } \\
\text { PRIVATE } \\
\text { CALL } \\
\text { SETUP } \\
\text { REQUEST }\end{array}$ & - & - & $\begin{array}{c}\text { UE B compares and } \\
\text { acknowledges difference } \\
\text { between Call Identifiers, } \\
\text { stores Call Identifier IE of } \\
\text { received message as call } \\
\text { identifier, MCPTT user ID } \\
\text { of caller IE as caller ID, own } \\
\text { MCPTT user ID as callee ID, } \\
\text { generates and sends } \\
\text { PRIVATE CALL REJECT, } \\
\text { and starts TFP7 } \\
\end{array}$ & 11.2.2.4.3.1 \\
\hline 6 & $\begin{array}{c}\text { T0 + } \\
\text { t_AB1 + } \\
\text { t_BA1 + } \\
\text { t_A1 + } \\
\text { t_AB2 + } \\
\text { t_BA2 }\end{array}$ & $\begin{array}{c}\text { UE A } \\
\text { receives } \\
\text { PRIVATE } \\
\text { CALL } \\
\text { REJECT }\end{array}$ & $\begin{array}{l}\text { P1: ignoring same } \\
\text { call ID }\end{array}$ & - & $\begin{array}{l}\text { UE A releases call control } \\
\text { state machine, stops TFP1 } \\
\text { and TFP2, and starts TFP7 }\end{array}$ & 11.2.2.4.2.7 \\
\hline
\end{tabular}

And the corresponding figure is Fig. 20:

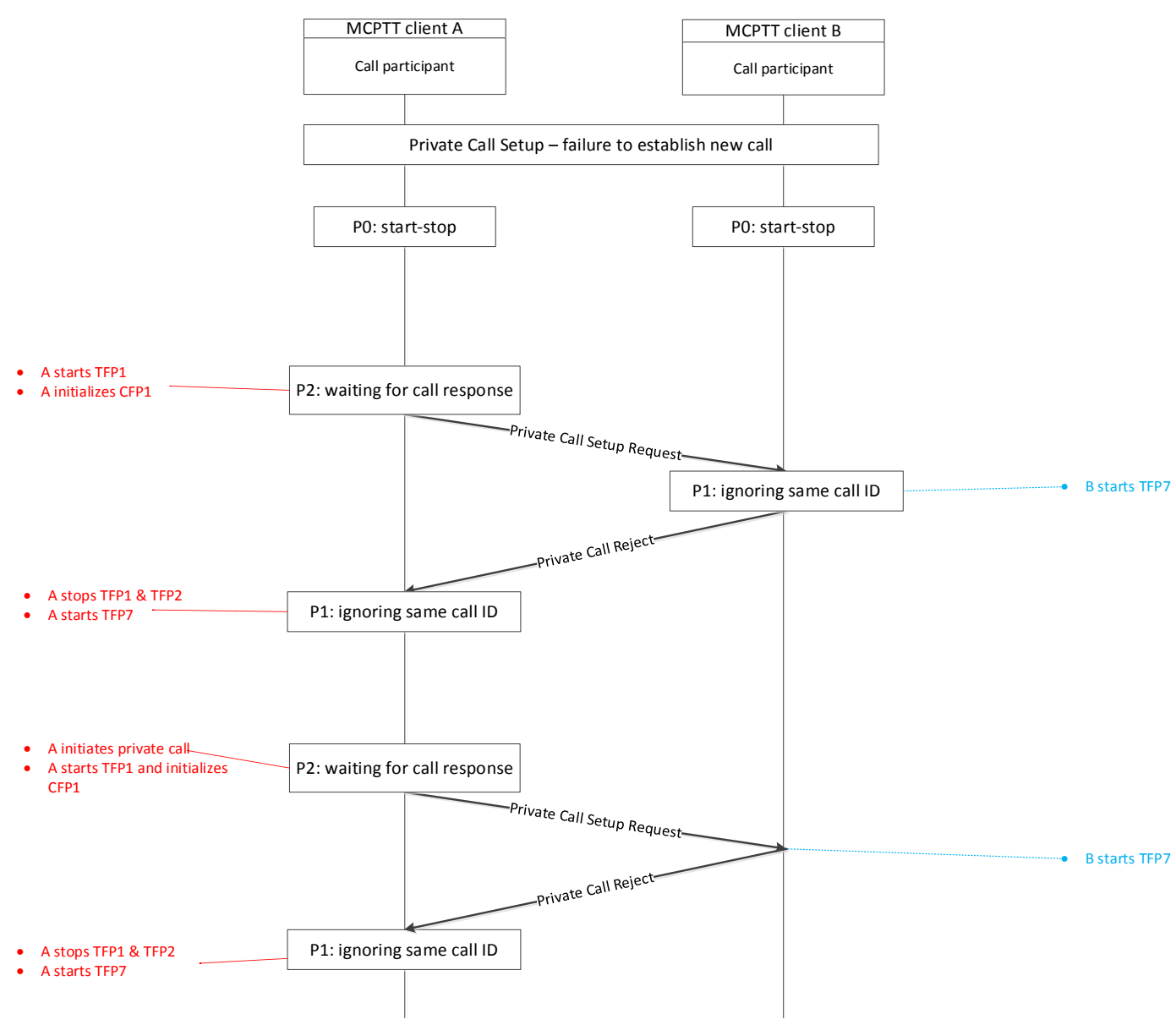

Figure 20: Private call setup - failure to establish a new call 


\subsubsection{Private call cancellation}

\subsubsection{Private call cancellation - cancel an ongoing call (normal)}

In this scenario a user, UE A, decides to cancel an ongoing private call as part of the usual normal call release (i.e., the user wants to end the private call).

Scenario purpose: To observe that a user can cancel an ongoing private call.

Table 59: Private call cancellation - cancel an ongoing call (normal)

\begin{tabular}{|c|c|c|c|c|c|c|}
\hline & Timeline & $\begin{array}{l}\text { Triggering } \\
\text { event }\end{array}$ & $\begin{array}{l}\text { UE A state } \\
\text { transition }\end{array}$ & $\begin{array}{l}\text { UE B state } \\
\text { transition }\end{array}$ & $\begin{array}{c}\text { Consequent } \\
\text { behavior }\end{array}$ & $\begin{array}{l}\text { Reference } \\
\text { for details }\end{array}$ \\
\hline 0 & - & - & $\begin{array}{l}\text { P4: part of } \\
\text { ongoing call }\end{array}$ & $\begin{array}{l}\text { P4: part of } \\
\text { ongoing call }\end{array}$ & - & - \\
\hline 1 & T0 & $\begin{array}{l}\text { UE A releases } \\
\text { the private call }\end{array}$ & $\begin{array}{c}\text { P3: waiting for } \\
\text { release } \\
\text { response }\end{array}$ & - & $\begin{array}{c}\text { UE A generates } \\
\text { and sends } \\
\text { PRIVATE CALL } \\
\text { RELEASE, starts } \\
\text { TFP3 and } \\
\text { initializes CFP3 } \\
\end{array}$ & 11.2.2.4.5.1 \\
\hline 2 & $\mathrm{~T} 0+\mathrm{t} \_\mathrm{AB} 1$ & $\begin{array}{l}\text { UE B receives } \\
\text { PRIVATE } \\
\text { CALL } \\
\text { RELEASE }\end{array}$ & - & $\begin{array}{l}\text { P1: ignoring } \\
\text { same call ID }\end{array}$ & $\begin{array}{l}\text { UE B generates } \\
\text { and sends } \\
\text { PRIVATE CALL } \\
\text { RELEASE ACK, } \\
\text { terminates media } \\
\text { session, releases } \\
\text { the call type } \\
\text { control state } \\
\text { machine and } \\
\text { starts TFP7 } \\
\end{array}$ & 11.2.2.4.5.4 \\
\hline 3 & $\begin{array}{c}\text { T0 + t_AB1 } \\
+ \text { t_BA1 }\end{array}$ & $\begin{array}{l}\text { UE A receives } \\
\text { PRIVATE } \\
\text { CALL } \\
\text { RELEASE ACK }\end{array}$ & $\begin{array}{l}\text { P1: ignoring } \\
\text { same call ID }\end{array}$ & - & $\begin{array}{c}\text { UE A terminates } \\
\text { the media } \\
\text { session, stops } \\
\text { TFP3 and starts } \\
\text { TFP7 }\end{array}$ & 11.2.2.4.5.5 \\
\hline
\end{tabular}

\subsubsection{Private call cancellation - cancel an ongoing call (timer expiry)}

In this scenario a user, UE A, decides to cancel an ongoing private call. However, those Private Call Release messages are lost and not received by UE B (out of range). Hence no response (i.e., Private Call Release Ack) message is received at UE A.

Scenario purpose: To observe that a user can cancel an ongoing private call using timer, due to a non-responding user (e.g., out of range). 
Table 60: Private call cancellation - cancel an ongoing call (timer expiry)

\begin{tabular}{|c|c|c|c|c|c|c|}
\hline & Timeline & $\begin{array}{c}\text { Triggering } \\
\text { event }\end{array}$ & $\begin{array}{l}\text { UE A state } \\
\text { transition }\end{array}$ & $\begin{array}{l}\text { UE B state } \\
\text { transition }\end{array}$ & $\begin{array}{c}\text { Consequent } \\
\text { behavior }\end{array}$ & $\begin{array}{l}\text { Reference } \\
\text { for details }\end{array}$ \\
\hline 0 & - & - & $\begin{array}{l}\text { P4: part of } \\
\text { ongoing call }\end{array}$ & $\begin{array}{l}\text { P4: part of } \\
\text { ongoing call }\end{array}$ & - & - \\
\hline 1 & T0 & $\begin{array}{l}\text { UE A } \\
\text { releases the } \\
\text { private call }\end{array}$ & $\begin{array}{c}\text { P3: waiting for } \\
\text { release } \\
\text { response }\end{array}$ & - & $\begin{array}{l}\text { UE A generates and } \\
\text { sends PRIVATE } \\
\text { CALL RELEASE, } \\
\text { starts TFP3 and } \\
\text { initializes CFP3 }\end{array}$ & 11.2 .2 .4 .5 .1 \\
\hline 2 & $\mathrm{~T} 0+\mathrm{TFP} 3$ & $\begin{array}{l}\text { UE A TFP3 } \\
\text { expires }\end{array}$ & - & - & $\begin{array}{l}\text { UE A generates and } \\
\text { sends PRIVATE } \\
\text { CALL RELEASE, } \\
\text { starts TFP3 and } \\
\text { increments CFP3 }\end{array}$ & 11.2 .2 .4 .5 .2 \\
\hline 3 & $\begin{array}{c}\text { T0 + } \\
\text { n*TFP3 }\end{array}$ & $\begin{array}{l}\text { UE A TFP3 } \\
\text { expires } \\
\text { (CFP3's } \\
\text { upper limit) }\end{array}$ & $\begin{array}{l}\text { P1: ignoring } \\
\text { same call ID-- }\end{array}$ & - & $\begin{array}{l}\text { UE A terminates the } \\
\text { media session, } \\
\text { releases the call type } \\
\text { control state machine } \\
\text { and starts TFP7 }\end{array}$ & 11.2 .2 .4 .5 .3 \\
\hline 4 & $\begin{array}{c}\text { T0 + TFP5 } \\
\text { (with TFP5 } \\
>n * T F P 3)\end{array}$ & $\begin{array}{l}\text { UE B TFP5 } \\
\text { expires }\end{array}$ & - & $\begin{array}{l}\text { P1: ignoring } \\
\text { same call ID }\end{array}$ & $\begin{array}{l}\text { UE B terminates the } \\
\text { media session, } \\
\text { releases the call type } \\
\text { control state machine } \\
\text { and starts TFP7 }\end{array}$ & 11.2 .2 .4 .5 .6 \\
\hline
\end{tabular}

And the corresponding figure is Fig. 21: 


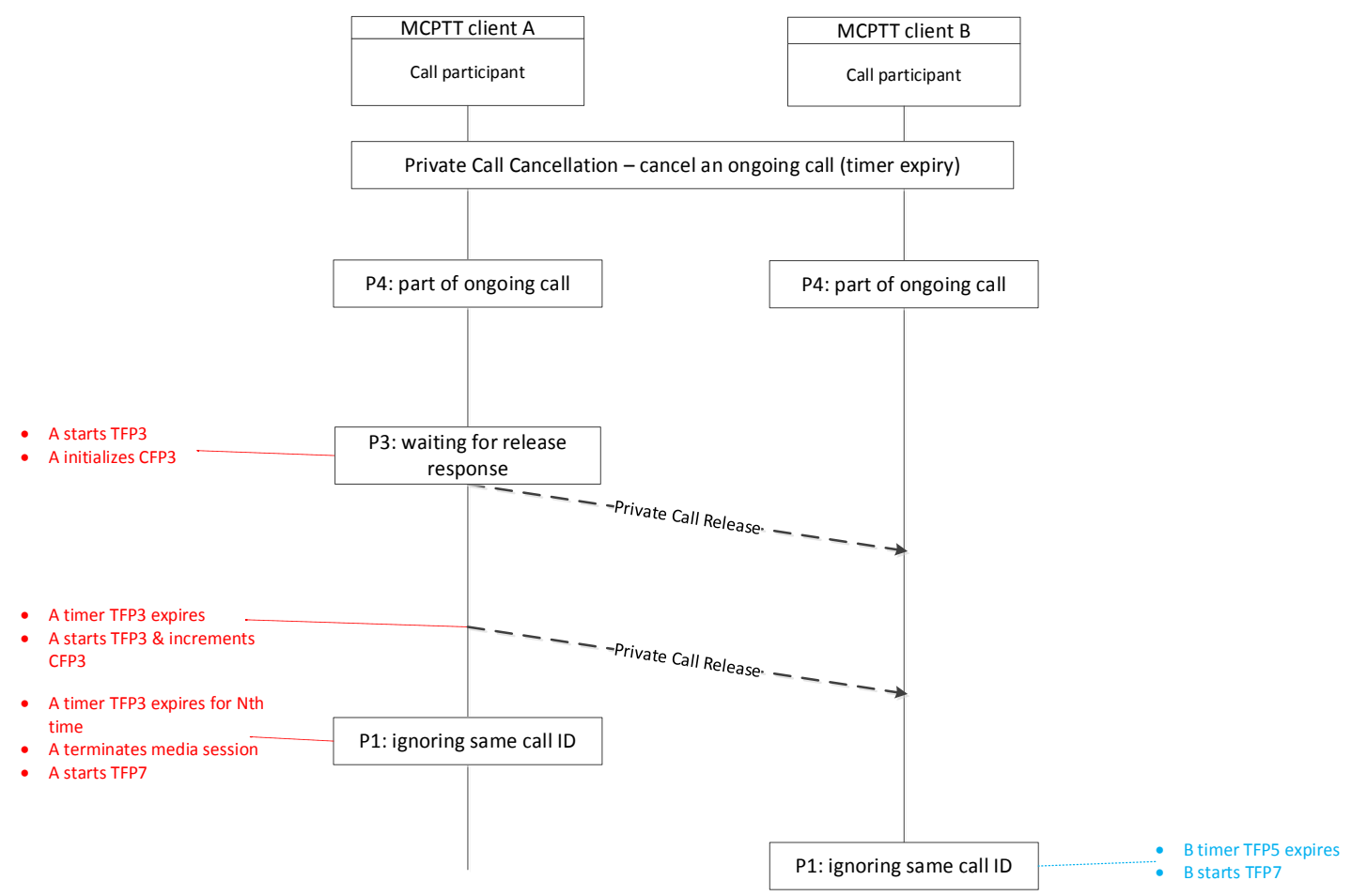

Figure 21: Private call cancellation - cancel an ongoing call (timer expiry)

\subsubsection{Private call expiration}

In this scenario a private call is ongoing. Timer TFP5 expires (lasted the maximum time for a private call), hence the call is terminated. The call is renewed afterwards.

Scenario purpose: To observe that a private call is terminated when the maximum duration for the call is reached (TFP5).

Table 61: Private call maximum duration expiration

\begin{tabular}{|c|c|c|c|c|c|c|}
\hline & Timeline & $\begin{array}{c}\text { Triggering } \\
\text { event }\end{array}$ & $\begin{array}{c}\text { UE A } \\
\text { state } \\
\text { transition }\end{array}$ & $\begin{array}{c}\text { UE B } \\
\text { state } \\
\text { transition }\end{array}$ & Consequent behavior & $\begin{array}{c}\text { Reference } \\
\text { for details }\end{array}$ \\
\hline 0 & - & $\begin{array}{c}\text { P4: part } \\
\text { of } \\
\text { ongoing } \\
\text { call }\end{array}$ & $\begin{array}{c}\text { P4: part } \\
\text { of } \\
\text { ongoing } \\
\text { call }\end{array}$ & & - \\
\hline 1 & T0 & $\begin{array}{c}\text { UE A TFP5 } \\
\text { expires }\end{array}$ & $\begin{array}{c}\text { P1: } \\
\text { ignoring } \\
\text { same call } \\
\text { ID }\end{array}$ & - & $\begin{array}{c}\text { UE A releases the call type } \\
\text { control state machine and } \\
\text { terminates the media session, and } \\
\text { starts TFP7 }\end{array}$ & $\mathbf{1 1 . 2 . 2 . 4 . 5 . 6}$ \\
\hline
\end{tabular}




\begin{tabular}{|c|c|c|c|c|c|c|}
\hline & Timeline & $\begin{array}{c}\text { Triggering } \\
\text { event }\end{array}$ & $\begin{array}{c}\text { UE A } \\
\text { state } \\
\text { transition }\end{array}$ & $\begin{array}{c}\text { UE B } \\
\text { state } \\
\text { transition }\end{array}$ & Consequent behavior & $\begin{array}{l}\text { Reference } \\
\text { for details }\end{array}$ \\
\hline 2 & $\mathrm{~T} 0+\mathrm{t} \_\mathrm{B} 1$ & $\begin{array}{l}\text { UE B TFP5 } \\
\text { expires }\end{array}$ & - & $\begin{array}{c}\text { P1: } \\
\text { ignoring } \\
\text { same call } \\
\text { ID }\end{array}$ & $\begin{array}{l}\text { UE B releases the call type } \\
\text { control state machine and } \\
\text { terminates the media session, and } \\
\text { starts TFP7 }\end{array}$ & 11.2.2.4.5.6 \\
\hline 3 & $\begin{array}{c}\mathrm{T} 0+ \\
\mathrm{t} \_\mathrm{A} 1 \\
(w i t h \\
t \_A 1>+ \\
\left.t \_B 1\right)\end{array}$ & $\begin{array}{l}\text { Indication } \\
\text { from UE A } \\
\text { to initiate a } \\
\text { private call }\end{array}$ & $\begin{array}{l}\text { P2: } \\
\text { waiting } \\
\text { for call } \\
\text { response }\end{array}$ & - & $\begin{array}{l}\text { UE A generates and stores Call } \\
\text { Identifier, MCPTT user ID, user } \\
\text { ID of the callee, user location, } \\
\text { offer SDP, creates call type } \\
\text { control state machine and } \\
\text { establishes end-to-end security (if } \\
\text { needed), stores commencement } \\
\text { mode as "MANUAL } \\
\text { COMMENCEMENT MODE", } \\
\text { generates and sends PRIVATE } \\
\text { CALL SETUP REQUEST, starts } \\
\text { TFP1 and initializes CFP1 }\end{array}$ & 11.2.2.4.2.1 \\
\hline 4 & $\begin{array}{c}\text { T0 + } \\
\text { t_A1 + } \\
\text { t_AB1 }\end{array}$ & $\begin{array}{c}\text { UE B } \\
\text { receives } \\
\text { PRIVATE } \\
\text { CALL } \\
\text { SETUP } \\
\text { REQUEST }\end{array}$ & - & $\begin{array}{c}\text { P5: } \\
\text { pending }\end{array}$ & $\begin{array}{l}\text { UE B stores Call Identifier IE as } \\
\text { call identifier, MCPTT user ID of } \\
\text { the caller IE as caller ID and own } \\
\text { MCPTT user ID as callee ID, } \\
\text { creates call type control state } \\
\text { machine, generates and stores } \\
\text { answer SDP, generates and sends } \\
\text { PRIVATE CALL RINGING, and } \\
\text { starts TFP2 }\end{array}$ & 11.2.2.4.4.1 \\
\hline 5 & $\begin{array}{l}\text { T0 + } \\
\text { t_A1 + } \\
\text { t_AB1 + } \\
\text { t_BA1 }\end{array}$ & $\begin{array}{c}\text { UE A } \\
\text { receives } \\
\text { PRIVATE } \\
\text { CALL } \\
\text { RINGING } \\
\end{array}$ & - & - & - & 11.2.2.4.2.3 \\
\hline
\end{tabular}

\section{2. $\quad$ Private call - call type control}

Note: Interaction dependence between private call control and private call type control.

\subsubsection{Enter private call}

In this scenario no ongoing private call is established yet. UE A eventually establishes a call. The stored emergency state is set to "False". The various messages sent are originating from the private call control state machine, not the private call type state machine.

Scenario purpose: To observe that the private call state type is entered upon establishment of a private call. 
Table 62: Enter private call

\begin{tabular}{|c|c|c|c|c|c|c|}
\hline & Timeline & $\begin{array}{c}\text { Triggering } \\
\text { event }\end{array}$ & $\begin{array}{l}\text { UE A state } \\
\text { transition }\end{array}$ & $\begin{array}{l}\text { UE B state } \\
\text { transition }\end{array}$ & Consequent behavior & $\begin{array}{l}\text { Reference } \\
\text { for details }\end{array}$ \\
\hline 0 & - & - & $\begin{array}{l}\text { Q0: waiting for } \\
\text { the call to be } \\
\text { established }\end{array}$ & $\begin{array}{l}\text { Q0: waiting for } \\
\text { the call to be } \\
\text { established }\end{array}$ & - & - \\
\hline 1 & T0 & $\begin{array}{c}\text { UE A } \\
\text { initiates a } \\
\text { Private } \\
\text { Call }\end{array}$ & - & - & $\begin{array}{l}\text { UE A sets current Call } \\
\text { Type to "PRIVATE } \\
\text { CALL" and stores } \\
\text { current ProSe per- } \\
\text { packet priority to } \\
\text { MCPTT off-network } \\
\text { private call } \\
\text { corresponding value ( } a \\
\text { PRIVATE CALL } \\
\text { SETUP REQUEST is } \\
\text { sent by UE A's basic } \\
\text { call control state } \\
\text { machine) } \\
\end{array}$ & 11.2.3.4.2 \\
\hline 2 & $\begin{array}{l}\mathrm{T} 0+ \\
\mathrm{t} \_\mathrm{AB} 1\end{array}$ & $\begin{array}{c}\text { UE B } \\
\text { receives } \\
\text { PRIVATE } \\
\text { CALL } \\
\text { SETUP } \\
\text { REQUEST } \\
\text { (message } \\
\text { sent from } \\
\text { the basic } \\
\text { call } \\
\text { control } \\
\text { state } \\
\text { machine) } \\
\end{array}$ & - & - & $\begin{array}{l}\text { UE B sets the current } \\
\text { call type to "PRIVATE } \\
\text { CALL" and sets } \\
\text { current ProSe per- } \\
\text { packet priority to } \\
\text { MCPTT off-network } \\
\text { private call } \\
\text { corresponding value }\end{array}$ & 11.2.3.4.3 \\
\hline 3 & $\begin{array}{c}\text { T0 + } \\
\text { t_AB1 + } \\
\text { t_BA1 }\end{array}$ & $\begin{array}{c}\text { UE A } \\
\text { receives } \\
\text { PRIVATE } \\
\text { CALL } \\
\text { ACCEPT } \\
\text { (message } \\
\text { sent from } \\
\text { the basic } \\
\text { call } \\
\text { control } \\
\text { state } \\
\text { machine) }\end{array}$ & $\begin{array}{l}\text { Q1: in-progress } \\
\text { private call }\end{array}$ & - & - & 11.2.3.4.4 \\
\hline
\end{tabular}




\begin{tabular}{|c|c|c|c|c|c|c|}
\hline & Timeline & $\begin{array}{c}\text { Triggering } \\
\text { event }\end{array}$ & $\begin{array}{l}\text { UE A state } \\
\text { transition }\end{array}$ & $\begin{array}{l}\text { UE B state } \\
\text { transition }\end{array}$ & Consequent behavior & $\begin{array}{l}\text { Reference } \\
\text { for details }\end{array}$ \\
\hline 4 & $\begin{array}{c}\text { T0 + } \\
\text { t_AB1 + } \\
\text { t_BA1 + } \\
\text { t_AB2 }\end{array}$ & $\begin{array}{c}\text { UE B } \\
\text { receives } \\
\text { PRIVATE } \\
\text { CALL } \\
\text { ACCEPT } \\
\text { ACK } \\
\text { (message } \\
\text { sent from } \\
\text { the basic } \\
\text { call } \\
\text { control } \\
\text { state } \\
\text { machine) }\end{array}$ & - & $\begin{array}{l}\text { Q1: in-progress } \\
\text { private call }\end{array}$ & - & 11.2.3.4.4 \\
\hline
\end{tabular}

\subsubsection{Enter private emergency call}

In this scenario no ongoing private call is established yet. UE A eventually establishes a call. The stored emergency state is set to "True".

Scenario purpose: To observe that the emergency private call state type is entered upon establishment of a private emergency call.

Table 63: Enter private emergency call

\begin{tabular}{|c|c|c|c|c|c|c|}
\hline & Timeline & $\begin{array}{c}\text { Triggering } \\
\text { event }\end{array}$ & $\begin{array}{l}\text { UE A state } \\
\text { transition }\end{array}$ & $\begin{array}{l}\text { UE B state } \\
\text { transition }\end{array}$ & Consequent behavior & $\begin{array}{l}\text { Reference } \\
\text { for details }\end{array}$ \\
\hline 0 & - & - & $\begin{array}{l}\text { Q0: waiting } \\
\text { for the call } \\
\text { to be } \\
\text { established }\end{array}$ & $\begin{array}{l}\text { Q0: waiting } \\
\text { for the call } \\
\text { to be } \\
\text { established }\end{array}$ & - & - \\
\hline 1 & T0 & $\begin{array}{l}\text { UE A initiates } \\
\text { a Private Call }\end{array}$ & - & - & $\begin{array}{l}\text { UE A sets current Call } \\
\text { Type to "EMERGENCY } \\
\text { PRIVATE CALL" and } \\
\text { stores current ProSe per- } \\
\text { packet priority to MCPTT } \\
\text { off-network private call } \\
\text { corresponding value ( } a \\
\text { PRIVATE CALL SETUP } \\
\text { REQUEST is sent by } \\
\text { UE A's basic call control } \\
\text { state machine) }\end{array}$ & 11.2.3.4.2 \\
\hline 2 & $\begin{array}{l}\text { T0 + } \\
\text { t_AB1 }\end{array}$ & $\begin{array}{l}\text { UE B receives } \\
\text { PRIVATE } \\
\text { CALL SETUP } \\
\text { REQUEST } \\
\text { (message sent } \\
\text { from the basic } \\
\text { call control } \\
\text { state machine) }\end{array}$ & - & - & $\begin{array}{l}\text { UE B sets the current call } \\
\text { type to "EMERGENCY } \\
\text { PRIVATE CALL" and sets } \\
\text { current ProSe per-packet } \\
\text { priority to MCPTT off- } \\
\text { network private call } \\
\text { corresponding value }\end{array}$ & 11.2.3.4.3 \\
\hline
\end{tabular}




\begin{tabular}{|c|c|c|c|c|c|c|}
\hline & Timeline & $\begin{array}{c}\text { Triggering } \\
\text { event }\end{array}$ & $\begin{array}{c}\text { UE A state } \\
\text { transition }\end{array}$ & $\begin{array}{c}\text { UE B state } \\
\text { transition }\end{array}$ & Consequent behavior & $\begin{array}{c}\text { Reference } \\
\text { for details }\end{array}$ \\
\hline 3 & $\begin{array}{c}\text { UE A receives }+ \\
\text { PRIVATE } \\
\text { CALL } \\
\text { t_BA1 }\end{array}$ & $\begin{array}{c}\text { ACCEPT } \\
\text { (message sent } \\
\text { from the basic } \\
\text { call control } \\
\text { state machine) }\end{array}$ & $\begin{array}{c}\text { Q2: in- } \\
\text { progress } \\
\text { emergency } \\
\text { private call }\end{array}$ & - & UE A starts TFP8 & $\mathbf{1 1 . 2 . 3 . 4 . 4}$ \\
\hline 4 & $\begin{array}{c}\text { UE B receives } \\
\text { PRIVATE } \\
\text { CALL }\end{array}$ & $\begin{array}{c}\text { CALL } \\
\text { t_AB1 }+ \\
\text { t_BA1 }+ \\
\text { ACCEPT ACK } \\
\text { (message sent } \\
\text { from the basic } \\
\text { call control } \\
\text { state machine) }\end{array}$ & - & $\begin{array}{c}\text { Q2: in- } \\
\text { progress } \\
\text { emergency } \\
\text { private call }\end{array}$ & UE B starts TFP8 & $\mathbf{1 1 . 2 . 3 . 4 . 4}$ \\
\hline
\end{tabular}

\subsubsection{Private call upgrade}

\subsubsection{Private call upgrade - upgrade call}

In this scenario an ongoing private call is already established. UE A decides to upgrade the call to an emergency private call.

Scenario purpose: To observe that a private call can be upgraded to an emergency private call, with corresponding state types.

Table 64: Private call upgrade - upgrade call

\begin{tabular}{|c|c|c|c|c|c|c|}
\hline Timeline & $\begin{array}{c}\text { Triggering } \\
\text { event }\end{array}$ & $\begin{array}{c}\text { UE A state } \\
\text { transition }\end{array}$ & $\begin{array}{c}\text { UE B state } \\
\text { transition }\end{array}$ & Consequent behavior & $\begin{array}{c}\text { Reference } \\
\text { for details }\end{array}$ \\
\hline 0 & - & - & $\begin{array}{c}\text { Q1: in- } \\
\text { progress } \\
\text { private call }\end{array}$ & $\begin{array}{c}\text { Q1: in- } \\
\text { progress } \\
\text { private call }\end{array}$ & & - \\
\hline
\end{tabular}




\begin{tabular}{|c|c|c|c|c|c|c|}
\hline & Timeline & $\begin{array}{c}\text { Triggering } \\
\text { event }\end{array}$ & $\begin{array}{l}\text { UE A state } \\
\text { transition }\end{array}$ & $\begin{array}{l}\text { UE B state } \\
\text { transition }\end{array}$ & Consequent behavior & $\begin{array}{l}\text { Reference } \\
\text { for details }\end{array}$ \\
\hline 1 & T0 & $\begin{array}{l}\text { UE A } \\
\text { upgrades } \\
\text { call to } \\
\text { Emergency } \\
\quad \text { Call }\end{array}$ & $\begin{array}{l}\text { Q2: in- } \\
\text { progress } \\
\text { emergency } \\
\text { private call }\end{array}$ & - & $\begin{array}{l}\text { UE A checks if emergency } \\
\text { leaf node from user profile is } \\
\text { set to "True", generates and } \\
\text { stores emergency offer SDP, } \\
\text { updates caller ID as own } \\
\text { MCPTT user ID and callee ID } \\
\text { as MCPTT user ID of the } \\
\text { other user, stores current user } \\
\text { location, sets current call type } \\
\text { to "EMERGENCY PRIVATE } \\
\text { CALL", generates and sends } \\
\text { PRIVATE CALL SETUP } \\
\text { REQUEST, sets ProSe per- } \\
\text { packet priority to } \\
\text { corresponding value, starts } \\
\text { TFP1 and initializes CFP1 }\end{array}$ & 11.2.3.4.5.1 \\
\hline 2 & $\begin{array}{l}\mathrm{T} 0+ \\
\mathrm{t} \_\mathrm{AB} 1\end{array}$ & $\begin{array}{l}\text { UE B } \\
\text { receives } \\
\text { PRIVATE } \\
\text { CALL } \\
\text { SETUP } \\
\text { REQUEST }\end{array}$ & - & $\begin{array}{l}\text { Q2: in- } \\
\text { progress } \\
\text { emergency } \\
\text { private call }\end{array}$ & $\begin{array}{l}\text { UE B generates and stores } \\
\text { emergency answer SDP, } \\
\text { updates caller ID with } \\
\text { MCPTT user ID of the caller } \\
\text { IE and callee ID with own } \\
\text { MCPTT user ID, generates } \\
\text { and sends PRIVATE CALL } \\
\text { ACCEPT, sets ProSe per- } \\
\text { packet priority to } \\
\text { corresponding value, sets } \\
\text { current call type to } \\
\text { "EMERGENCY PRIVATE } \\
\text { CALL", and starts TFP8 }\end{array}$ & 11.2.3.4.5.6 \\
\hline 3 & $\begin{array}{c}\mathrm{T} 0+ \\
\mathrm{t} \_\mathrm{AB} 1+ \\
\mathrm{t} \_\mathrm{BA} 1\end{array}$ & $\begin{array}{l}\text { UE A } \\
\text { receives } \\
\text { PRIVATE } \\
\text { CALL } \\
\text { ACCEPT }\end{array}$ & - & - & $\begin{array}{l}\text { UE A stores SDP answer IE as } \\
\text { emergency SDP answer, } \\
\text { generates and sends PRIVATE } \\
\text { CALL ACCEPT ACK, } \\
\text { establishes media session, } \\
\text { stops TFP1 and starts TFP8 } \\
\end{array}$ & 11.2.3.4.5.3 \\
\hline
\end{tabular}

\subsubsection{Private call upgrade - rejected upgrade call}

In this scenario an ongoing private call is already established. UE A decides to upgrade the call to an emergency private call. The media session cannot be established on UE B's side hence the upgrade is rejected. A TFP1 timer expiration occurs due to the first private call setup request message being lost.

Scenario purpose: To observe that a private call upgrade can be rejected by the called user. 
Table 65: Private call upgrade - rejected upgrade call

\begin{tabular}{|c|c|c|c|c|c|c|}
\hline & Timeline & $\begin{array}{c}\text { Triggering } \\
\text { event }\end{array}$ & $\begin{array}{l}\text { UE A state } \\
\text { transition }\end{array}$ & $\begin{array}{l}\text { UE B state } \\
\text { transition }\end{array}$ & Consequent behavior & $\begin{array}{l}\text { Reference } \\
\text { for details }\end{array}$ \\
\hline 0 & - & - & $\begin{array}{c}\text { Q1: in- } \\
\text { progress } \\
\text { private call }\end{array}$ & $\begin{array}{c}\text { Q1: in- } \\
\text { progress } \\
\text { private call }\end{array}$ & - & - \\
\hline 1 & T0 & $\begin{array}{l}\text { UE A } \\
\text { upgrades } \\
\text { call to } \\
\text { Emergency } \\
\text { Call }\end{array}$ & $\begin{array}{l}\text { Q2: in- } \\
\text { progress } \\
\text { emergency } \\
\text { private call }\end{array}$ & - & $\begin{array}{l}\text { UE A checks if emergency } \\
\text { leaf node from user profile is } \\
\text { set to "True", generates and } \\
\text { stores emergency offer SDP, } \\
\text { updates caller ID as own } \\
\text { MCPTT user ID and callee ID } \\
\text { as MCPTT user ID of the } \\
\text { other user, stores current user } \\
\text { location, sets current call type } \\
\text { to "EMERGENCY PRIVATE } \\
\text { CALL", generates and sends } \\
\text { PRIVATE CALL SETUP } \\
\text { REQUEST, sets ProSe per- } \\
\text { packet priority to } \\
\text { corresponding value, starts } \\
\text { TFP1 and initializes CFP1 }\end{array}$ & 11.2.3.4.5.1 \\
\hline 2 & $\begin{array}{l}\text { T0 + } \\
\text { TFP1 }\end{array}$ & $\begin{array}{c}\text { UE A TFP1 } \\
\text { expires }\end{array}$ & - & - & $\begin{array}{c}\text { UE A may update user } \\
\text { location, generates and sends } \\
\text { PRIVATE CALL SETUP } \\
\text { REQUEST, starts TFP1 and } \\
\text { increments CFP1 } \\
\end{array}$ & 11.2.3.4.5.2 \\
\hline 3 & $\begin{array}{l}\text { T0 + } \\
\text { TFP1 + } \\
\text { t_AB1 }\end{array}$ & $\begin{array}{l}\text { UE B } \\
\text { receives } \\
\text { PRIVATE } \\
\text { CALL } \\
\text { SETUP } \\
\text { REQUEST }\end{array}$ & - & - & $\begin{array}{l}\text { UE B sets call identifier IE } \\
\text { with the call identifier in the } \\
\text { received message, sets } \\
\text { MCPTT user ID of the caller } \\
\text { IE and of the callee IE, sets } \\
\text { Reason IE as either "FAILED" } \\
\text { or "MEDIA FAILURE", } \\
\text { generates and sends PRIVATE } \\
\text { CALL REJECT }\end{array}$ & 11.2.3.4.5.6 \\
\hline 4 & $\begin{array}{c}\text { T0 + } \\
\text { TFP1 + } \\
\text { t_AB1 + } \\
\text { t_BA1 }\end{array}$ & $\begin{array}{l}\text { UE A } \\
\text { receives } \\
\text { PRIVATE } \\
\text { CALL } \\
\text { REJECT }\end{array}$ & $\begin{array}{c}\text { Q1: in- } \\
\text { progress } \\
\text { private call }\end{array}$ & - & $\begin{array}{c}\text { UE A sets ProSe per-packet } \\
\text { priority to corresponding } \\
\text { value, sets current call type to } \\
\text { "PRIVATE CALL" and stops } \\
\text { TFP1 }\end{array}$ & 11.2.3.4.5.4 \\
\hline
\end{tabular}

And the corresponding figure is Fig. 22: 


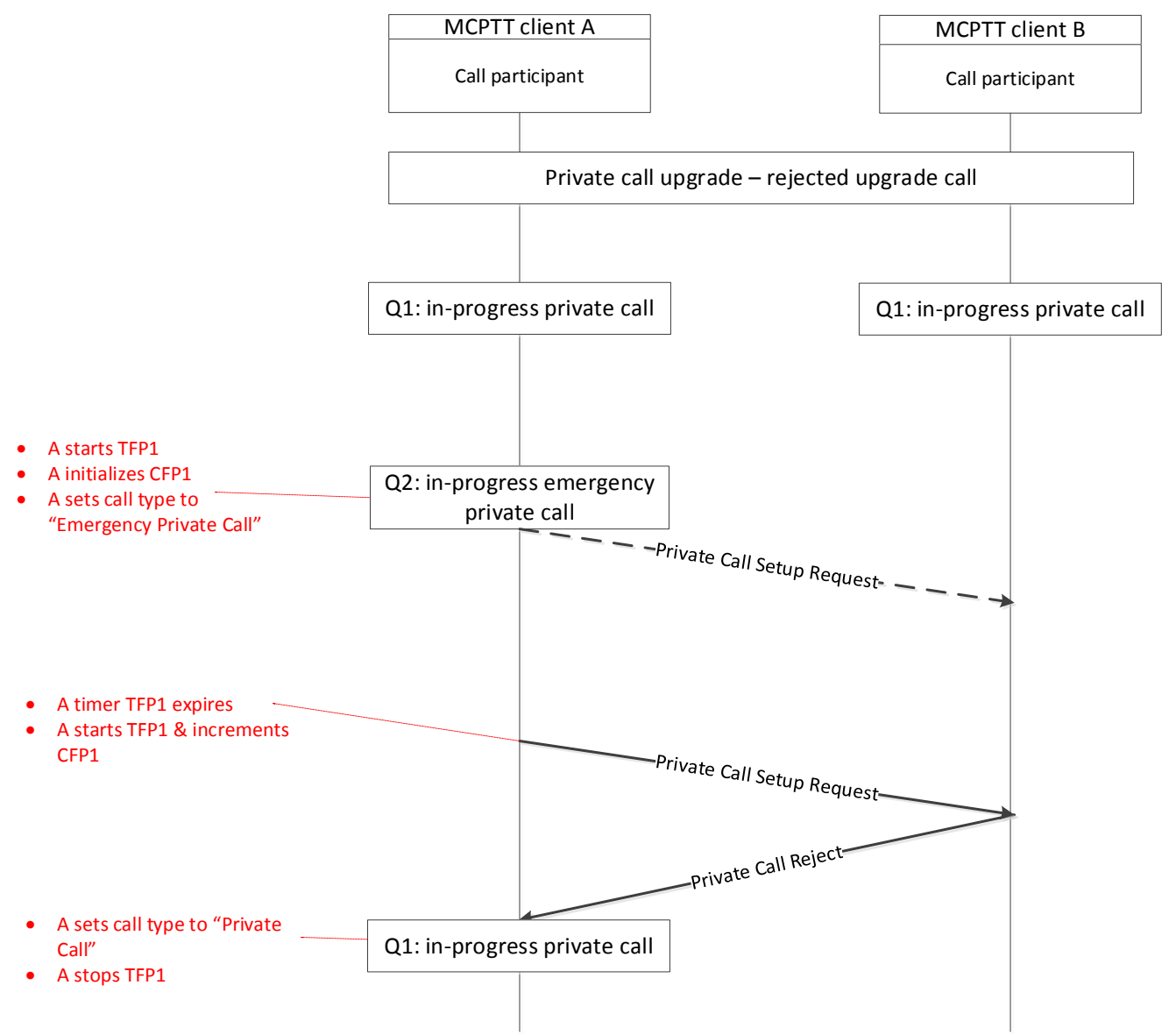

Figure 22: Private call upgrade - rejected upgrade call

\subsubsection{Private call upgrade - failed upgrade call}

In this scenario an ongoing private call is already established. UE A decides to upgrade the call to an emergency private call. However, in the meantime UE A and UE B get out of range of each other. UE B does not respond to the upgrade, hence UE A releases the call.

Scenario purpose: To observe that a private call upgrade fails if the user does not respond (e.g., out of range), and results in the call being released by the callee and called party receives an internal indication to release the call.

Table 66: Private call upgrade - failed upgrade call

\begin{tabular}{|c|c|c|c|c|c|c|}
\hline Timeline & $\begin{array}{c}\text { Triggering } \\
\text { event }\end{array}$ & $\begin{array}{c}\text { UE A state } \\
\text { transition }\end{array}$ & $\begin{array}{c}\text { UE B state } \\
\text { transition }\end{array}$ & Consequent behavior & $\begin{array}{c}\text { Reference } \\
\text { for details }\end{array}$ \\
\hline 0 & - & - & $\begin{array}{c}\text { Q1: in- } \\
\text { progress } \\
\text { private call }\end{array}$ & $\begin{array}{c}\text { Q1: in- } \\
\text { progress } \\
\text { private call }\end{array}$ & & - \\
\hline
\end{tabular}




\begin{tabular}{|c|c|c|c|c|c|c|}
\hline & Timeline & $\begin{array}{c}\text { Triggering } \\
\text { event }\end{array}$ & $\begin{array}{l}\text { UE A state } \\
\text { transition }\end{array}$ & $\begin{array}{l}\text { UE B state } \\
\text { transition }\end{array}$ & Consequent behavior & $\begin{array}{l}\text { Reference } \\
\text { for details }\end{array}$ \\
\hline 1 & T0 & $\begin{array}{l}\text { UE A } \\
\text { upgrades } \\
\text { call to } \\
\text { Emergency } \\
\text { Call }\end{array}$ & $\begin{array}{l}\text { Q2: in- } \\
\text { progress } \\
\text { emergency } \\
\text { private call }\end{array}$ & - & $\begin{array}{l}\text { UE A checks if } \\
\text { emergency leaf node } \\
\text { from user profile is set to } \\
\text { "True", generates and } \\
\text { stores emergency offer } \\
\text { SDP, updates caller ID } \\
\text { as own MCPTT user ID } \\
\text { and callee ID as MCPTT } \\
\text { user ID of the other user, } \\
\text { stores current user } \\
\text { location, sets current call } \\
\text { type to "EMERGENCY } \\
\text { PRIVATE CALL"", } \\
\text { generates and sends } \\
\text { PRIVATE CALL } \\
\text { SETUP REQUEST, sets } \\
\text { ProSe per-packet priority } \\
\text { to corresponding value, } \\
\text { starts TFP1 and } \\
\text { initializes CFP1 } \\
\end{array}$ & 11.2.3.4.5.1 \\
\hline 2 & $\begin{array}{l}\text { T0 + } \\
\text { TFP1 }\end{array}$ & $\begin{array}{l}\text { UE A TFP1 } \\
\text { expires }\end{array}$ & - & - & $\begin{array}{l}\text { UE A may update user } \\
\text { location, generates and } \\
\text { sends PRIVATE CALL } \\
\text { SETUP REQUEST, } \\
\text { starts TFP1 and } \\
\text { increments CFP1 }\end{array}$ & 11.2.3.4.5.2 \\
\hline 3 & $\begin{array}{c}\mathrm{T} 0+ \\
\mathrm{n} * \mathrm{TFP} 1\end{array}$ & $\begin{array}{l}\text { UE A TFP1 } \\
\text { expires } \\
\text { (CFP1 at its } \\
\text { upper limit) }\end{array}$ & $\begin{array}{l}\text { Q0: waiting } \\
\text { for the call to } \\
\text { be established }\end{array}$ & - & $\begin{array}{l}\text { UE A releases stored } \\
\text { current call type, releases } \\
\text { ProSe per-packet priority }\end{array}$ & 11.2.3.4.5.5 \\
\hline 4 & $\begin{array}{c}\mathrm{T} 0+ \\
\mathrm{t} \_\mathrm{B} 1 \\
(w i t h \\
t \_B 1> \\
\left.n^{*} T F P 1\right)\end{array}$ & $\begin{array}{l}\text { UE B } \\
\text { releases the } \\
\text { call }\end{array}$ & - & $\begin{array}{l}\text { Q0: waiting for } \\
\text { the call to be } \\
\text { established }\end{array}$ & $\begin{array}{l}\text { UE B releases stored } \\
\text { current call type, releases } \\
\text { ProSe per-packet priority }\end{array}$ & 11.2.3.4.7 \\
\hline
\end{tabular}

\subsubsection{Private emergency call downgrade}

\subsubsection{Private emergency call downgrade - downgrade call TFP6 expiry}


In this scenario an ongoing emergency private call is already established. UE A decides to downgrade the call to a private call. Timer TFP6 expires since the first PRIVATE CALL EMERGENCY CANCEL message is lost.

Scenario purpose: To observe that an ongoing emergency private call can be downgraded to a private call, even with the loss of a single message (PRIVATE CALL EMERGENCY CANCEL).

Table 67: Private emergency call downgrade - downgrade call TFP6 expiry

\begin{tabular}{|c|c|c|c|c|c|c|}
\hline & Timeline & $\begin{array}{c}\text { Triggering } \\
\text { event }\end{array}$ & $\begin{array}{l}\text { UE A state } \\
\text { transition }\end{array}$ & $\begin{array}{l}\text { UE B state } \\
\text { transition }\end{array}$ & Consequent behavior & $\begin{array}{l}\text { Reference } \\
\text { for details }\end{array}$ \\
\hline 0 & - & - & $\begin{array}{c}\text { Q2: in- } \\
\text { progress } \\
\text { emergency } \\
\text { private call }\end{array}$ & $\begin{array}{c}\text { Q2: in- } \\
\text { progress } \\
\text { emergency } \\
\text { private call }\end{array}$ & - & - \\
\hline 1 & T0 & $\begin{array}{l}\text { UE A cancels the } \\
\text { emergency } \\
\text { private call }\end{array}$ & $\begin{array}{c}\text { Q1: in- } \\
\text { progress } \\
\text { private call }\end{array}$ & - & $\begin{array}{c}\text { UE A generates and } \\
\text { sends PRIVATE CALL } \\
\text { EMERGENCY } \\
\text { CANCEL, sets stored } \\
\text { current call type to } \\
\text { "PRIVATE CANCEL", } \\
\text { stops TFP8, initializes } \\
\text { CFP6 and starts TFP6 }\end{array}$ & 11.2.3.4.6.1 \\
\hline 2 & $\begin{array}{l}\text { T0 + } \\
\text { TFP6 }\end{array}$ & $\begin{array}{l}\text { UE A TFP6 } \\
\text { expires }\end{array}$ & - & - & $\begin{array}{c}\text { UE A generates and } \\
\text { sends PRIVATE CALL } \\
\text { EMERGENCY } \\
\text { CANCEL, starts TFP6 } \\
\text { and increments CFP6 }\end{array}$ & 11.2.3.4.6.2 \\
\hline 3 & $\begin{array}{l}\text { T0 + } \\
\text { TFP6 + } \\
\text { t_AB1 }\end{array}$ & $\begin{array}{l}\text { UE B receives } \\
\text { PRIVATE } \\
\text { CALL } \\
\text { EMERGENCY } \\
\text { CANCEL }\end{array}$ & - & $\begin{array}{l}\text { Q1: in- } \\
\text { progress } \\
\text { private call }\end{array}$ & $\begin{array}{c}\text { UE B generates and } \\
\text { sends PRIVATE CALL } \\
\text { EMERGENCY CANCEL } \\
\text { ACK, establishes a media } \\
\text { session based on the SDP } \\
\text { body of stored answer } \\
\text { SDP, sets current call } \\
\text { type to "PRIVATE } \\
\text { CALL", sets ProSe per- } \\
\text { packet priority to } \\
\text { corresponding value, and } \\
\text { stops TFP8 } \\
\end{array}$ & 11.2.3.4.6.5 \\
\hline 4 & $\begin{array}{c}\text { T0 + } \\
\text { TFP6 + } \\
\text { t_AB1 + } \\
\text { t_BA1 }\end{array}$ & $\begin{array}{l}\text { UE A receives } \\
\text { PRIVATE } \\
\text { CALL } \\
\text { EMERGENCY } \\
\text { CANCEL ACK }\end{array}$ & - & - & $\begin{array}{l}\text { UE A sets ProSe per- } \\
\text { packet priority to } \\
\text { corresponding value, } \\
\text { establishes a media } \\
\text { session based on the SDP } \\
\text { body of stored answer } \\
\text { SDP, and stops TFP6 }\end{array}$ & 11.2.3.4.6.3 \\
\hline
\end{tabular}


And the corresponding figure is Fig. 23:

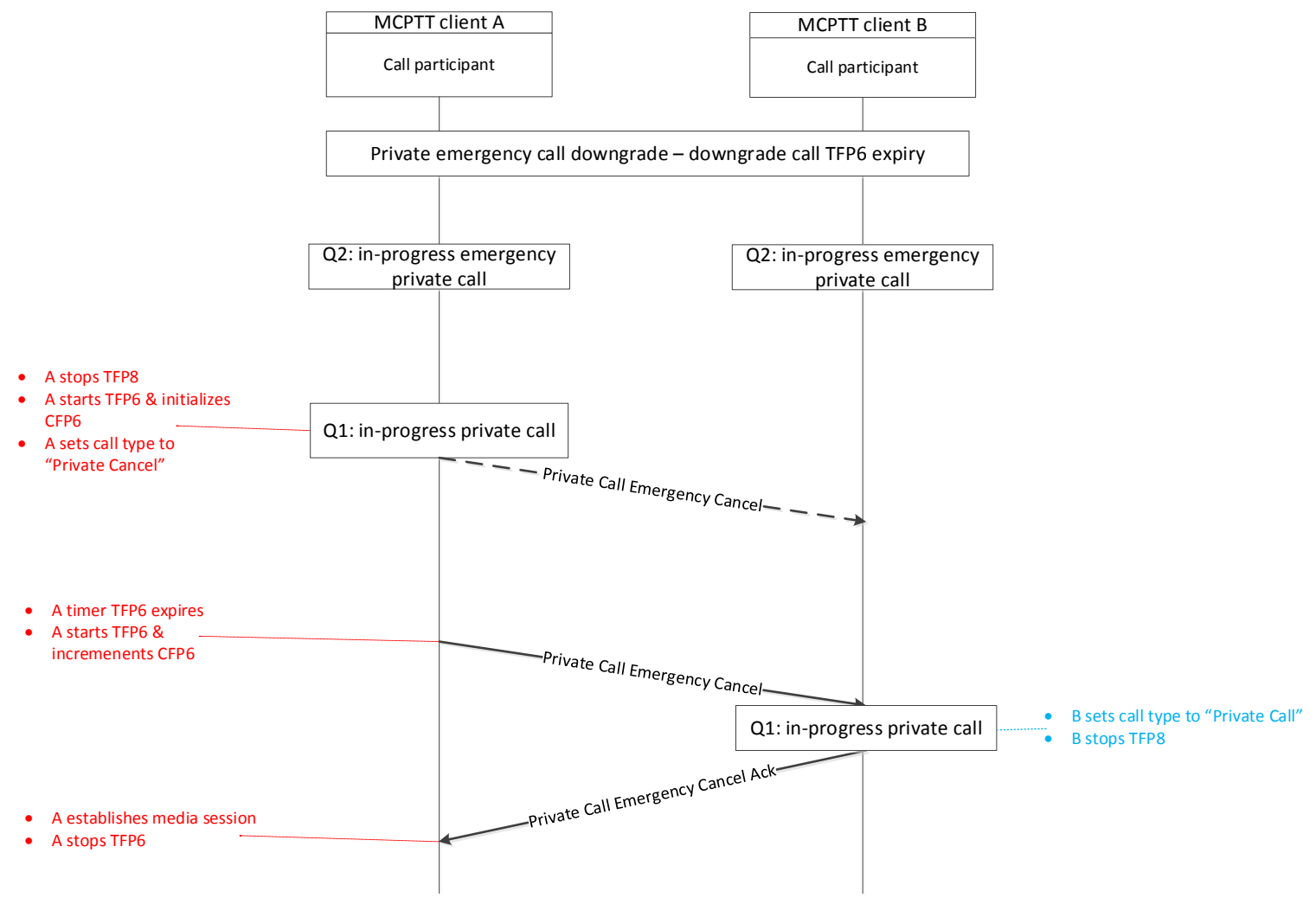

Figure 23: Private emergency call downgrade - downgrade call TFP6 expiry

\subsubsection{Private emergency call downgrade - downgrade call out of range}

In this scenario an ongoing emergency private call is already established. UE A decides to downgrade the call to a private call. However, the "PRIVATE CALL EMERGENCY CANCEL" messages sent by UE A are lost (UE B is out of range) during this scenario. Thus, UE B does not respond to the downgrade, so UE A releases the call.

Scenario purpose: To observe that, when downgrading an ongoing emergency private call to a basic private call, if both users are out of range then the private call eventually gets released by UE A. 
Table 68: Private emergency call downgrade - downgrade call out of range

\begin{tabular}{|c|c|c|c|c|c|c|}
\hline & Timeline & $\begin{array}{c}\text { Triggering } \\
\text { event }\end{array}$ & $\begin{array}{l}\text { UE A state } \\
\text { transition }\end{array}$ & $\begin{array}{l}\text { UE B state } \\
\text { transition }\end{array}$ & Consequent behavior & $\begin{array}{l}\text { Reference } \\
\text { for details }\end{array}$ \\
\hline 0 & - & - & $\begin{array}{l}\text { Q2: in- } \\
\text { progress } \\
\text { emergency } \\
\text { private call }\end{array}$ & $\begin{array}{l}\text { Q2: in- } \\
\text { progress } \\
\text { emergency } \\
\text { private call }\end{array}$ & - & - \\
\hline 1 & T0 & $\begin{array}{l}\text { UE A cancels } \\
\text { the emergency } \\
\text { private call }\end{array}$ & $\begin{array}{c}\text { Q1: in- } \\
\text { progress } \\
\text { private call }\end{array}$ & - & $\begin{array}{c}\text { UE A generates and } \\
\text { sends PRIVATE CALL } \\
\text { EMERGENCY } \\
\text { CANCEL, sets stored } \\
\text { current call type to } \\
\text { "PRIVATE CANCEL", } \\
\text { stops TFP8, initializes } \\
\text { CFP6 and starts TFP6 } \\
\end{array}$ & 11.2.3.4.6.1 \\
\hline 2 & $\begin{array}{c}\text { T0 + } \\
\text { TFP6 }\end{array}$ & $\begin{array}{l}\text { UE A TFP6 } \\
\text { expires }\end{array}$ & - & - & $\begin{array}{c}\text { UE A generates and } \\
\text { sends PRIVATE CALL } \\
\text { EMERGENCY } \\
\text { CANCEL, starts TFP6 } \\
\text { and increments CFP6 }\end{array}$ & 11.2.3.4.6.2 \\
\hline 3 & $\begin{array}{c}\mathrm{T} 0+ \\
\mathrm{n} * \mathrm{TFP} 6\end{array}$ & $\begin{array}{l}\text { UE A TFP6 } \\
\text { expires (with } \\
\text { value of CFP6 } \\
\text { at its upper } \\
\text { limit) }\end{array}$ & $\begin{array}{l}\text { Q0: waiting } \\
\text { for the call } \\
\text { to be } \\
\text { established }\end{array}$ & - & $\begin{array}{c}\text { UE A releases stored } \\
\text { current call type, releases } \\
\text { stored ProSe per-packet } \\
\text { priority }\end{array}$ & 11.2.3.4.6.4 \\
\hline
\end{tabular}

And the corresponding figure is Fig. 24: 


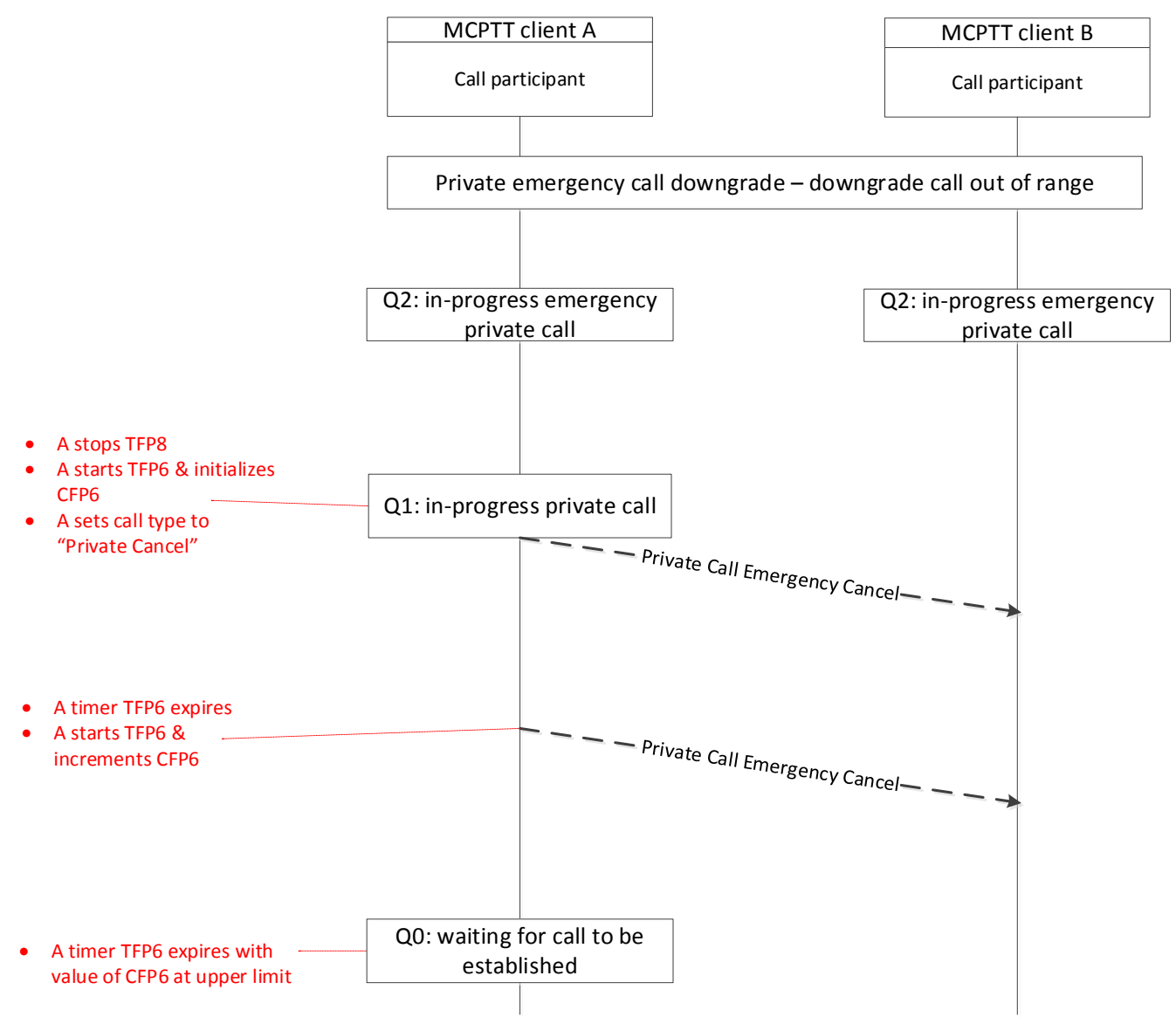

Figure 24: Private emergency call downgrade - downgrade call out of range

\subsubsection{Private emergency call downgrade - downgrade call TFP8 expiry}

In this scenario an ongoing emergency private call is already established. Timer TFP8 expires at UE A and at UE B, so both UE A and UE B downgrade the call to a private call.

Scenario purpose: To observe that an ongoing emergency private call gets downgraded once the appropriate implicit timer (TFP8) expires.

Table 69: Private emergency call downgrade - downgrade call TFP8 expiry

\begin{tabular}{|c|c|c|c|c|c|c|}
\hline & Timeline & $\begin{array}{c}\text { Triggering } \\
\text { event }\end{array}$ & $\begin{array}{c}\text { UE A state } \\
\text { transition }\end{array}$ & $\begin{array}{c}\text { UE B state } \\
\text { transition }\end{array}$ & Consequent behavior & $\begin{array}{c}\text { Reference } \\
\text { for details }\end{array}$ \\
\hline 0 & - & - & $\begin{array}{c}\text { Q2: in- } \\
\text { progress } \\
\text { emergency } \\
\text { private call }\end{array}$ & $\begin{array}{c}\text { Q2: in- } \\
\text { progress } \\
\text { emergency } \\
\text { private call }\end{array}$ & & - \\
\hline
\end{tabular}




\begin{tabular}{|c|c|c|c|c|c|c|}
\hline & Timeline & $\begin{array}{c}\text { Triggering } \\
\text { event }\end{array}$ & $\begin{array}{l}\text { UE A state } \\
\text { transition }\end{array}$ & $\begin{array}{l}\text { UE B state } \\
\text { transition }\end{array}$ & Consequent behavior & $\begin{array}{l}\text { Reference } \\
\text { for details }\end{array}$ \\
\hline 1 & T0 & $\begin{array}{c}\text { UE A TFP8 } \\
\text { expires }\end{array}$ & $\begin{array}{c}\text { Q1: in- } \\
\text { progress } \\
\text { private call }\end{array}$ & - & $\begin{array}{l}\text { UE A establishes a media } \\
\text { session based on SDP body } \\
\text { of stored answer SDP, sets } \\
\text { ProSe per-packet priority to } \\
\text { corresponding value, and } \\
\text { sets stored current call type } \\
\text { to "PRIVATE CALL" }\end{array}$ & 11.2.3.4.6A \\
\hline 2 & $\begin{array}{l}\text { T0 + } \\
\text { t_B1 }\end{array}$ & $\begin{array}{c}\text { UE B TFP8 } \\
\text { expires }\end{array}$ & - & $\begin{array}{c}\text { Q1: in- } \\
\text { progress } \\
\text { private call }\end{array}$ & $\begin{array}{l}\text { UE B establishes a media } \\
\text { session based on SDP body } \\
\text { of stored answer SDP, sets } \\
\text { ProSe per-packet priority to } \\
\text { corresponding value, and } \\
\text { sets stored current call type } \\
\text { to "PRIVATE CALL" }\end{array}$ & 11.2.3.4.6A \\
\hline
\end{tabular}




\section{Summary and future work}

Test scenarios presented in this document are designed based on 3GPP's MCPTT off-network mode protocols strictly, with the objective to help the verification of protocol implementations. Benefits of these test scenarios are at least three folds:

$\checkmark$ First, and the motivation of the effort, is that test scenarios are used to verify the ns-3 simulation model that our group developed. The simulator passed all tests.

$\checkmark$ Second, during the process of test scenario design and simulation model verification, the completeness and correctness of 3GPP's MCPTT off-network mode operations were examined in great details. In the past one and a half years, our group has identified many errors and discrepancies in the MCPTT floor control and call control protocols, and, as a result, have submitted several CRs [8-19] addressing off-network mode operations to the 3GPP CT1 task group international meetings. All documents were well received, as all corrections were accepted.

$\checkmark$ Third, but not least, it is possible to utilize these test scenarios to verify the MCPTT protocol implementations in other embodiments, e.g., prototype and commercial devices. It is also possible to develop more specific test scenarios that may be adopted by 3GPP, especially the sequence of message exchanges over the air, which are more observable externally based on the figures that accompany several test scenarios.

The above benefits may accelerate the deployment of MCV over LTE, especially for the outof-coverage communication situation for public safety users. In the future, we plan to update existing test scenarios and simulation models to conform with 3GPP standards as progress is made.

\section{Acknowledgments}

The authors would like to thank Richard Rouil and David Cypher of Wireless Networks Division in Communications Technology Laboratory at National Institute of Standards and Technology for their technical discussions and suggestions, whose knowledge and insights have accelerated the achievement of the task and motivated the completion of this document. 


\section{References}

[1] NPSTC Public Safety Communications Report, "Public Safety Broadband Push-to-Talk over Long Term Evolution Requirements,” July 2013.

[2] 3GPP TS 22.179 v13.3.0, "Mission Critical Push To Talk (MCPTT) over LTE; Stage 1 (Release 13)”, December 2015.

[3] 3GPP TS 23.179 v13.5.0, "Functional architecture and information flows to support mission critical communication services; Stage 2 (Release 13)”, March 2017.

[4] 3GPP TS 24.379 v14.2.0, “Mission Critical Push To Talk (MCPTT) call control; Protocol specification (Release 14)”, June 2017.

[5] 3GPP TS 24.380 v14.3.0, “Mission Critical Push To Talk (MCPTT) media plane control; Protocol specification (Release 14)”, June 2017.

[6] 3GPP TS 24.381 v13.4.0, “Mission Critical Push To Talk (MCPTT) group management; Protocol specification (Release 13)”, March 2017.

[7] 3GPP TS 24.384 v13.4.0, "Mission Critical Push To Talk (MCPTT) configuration management; Protocol specification (Release 13)”, March 2017.

[8] US Department of Commerce, CT1 \#99, “Corrections needed for alignment within TS”, C1-163707 (revision of C1-163265), Tenerife, Spain, July 2016

[9] US Department of Commerce, FirstNet, CT1 \#105, “Corrections to off-network floor control procedures (Rel-13)”, C1-173438 (revision of C1-172949), Krakow, Poland, August 2017

[10] US Department of Commerce, FirstNet, CT1 \#105, "Corrections to off-network floor control procedures (Rel-14)”, C1-173439 (revision of C1-172949), Krakow, Poland, August 2017

[11] US Department of Commerce, FirstNet, CT1 \#105, "Explicit corrections to off-network floor control procedures and steps (Rel-13)”, C1-173440 (revision of C1-172950), Krakow, Poland, August 2017

[12] US Department of Commerce, FirstNet, CT1 \#105, "Explicit corrections to off-network floor control procedures and steps (Rel-14)”, C1-173441 (revision of C1-172950), Krakow, Poland, August 2017

[13] US Department of Commerce, FirstNet, CT1 \#105, "Corrections to off-network call control procedures (Rel-13)”, C1-173449 (revision of C1-173150), Krakow, Poland, August 2017

[14] US Department of Commerce, FirstNet, CT1 \#105, "Corrections to off-network call control procedures (Rel-14)”, C1-173450 (revision of C1-173150), Krakow, Poland, August 2017

[15] US Department of Commerce, CT1 \#107, “Off-network call type control procedures: merge of two calls (Rel-13)”, C1-175223 (revision of C1-174780), Reno, USA, November 2017

[16] US Department of Commerce, CT1 \#107, “Off-network call type control procedures: merge of two calls (Rel-14)”, C1-175224 (revision of C1-174780), Reno, USA, November 2017

[17] US Department of Commerce, CT1 \#107, “Off-network Broadcast group call procedures (Rel-13)”, C1-175225 (revision of C1-174781), Reno, USA, November 2017 [18] US Department of Commerce, CT1 \#107, “Off-network Broadcast group call procedures (Rel-14)”, C1-175226 (revision of C1-174781), Reno, USA, November 2017 
[19] US Department of Commerce, CT1 \#107, “Companion document for Off-network Broadcast Group Call procedures updates”, C1-174782, Reno, USA, November 2017 


\section{Appendix: Scenario usages}

This appendix gives a few examples for using the scenarios described in this document in other areas of expertise, such as software testing, conformance testing, and interoperability testing.

\section{- Software testing:}

When using the scenarios for software tests, they can be used to derive white box tests, because some scenarios lack the necessary control and events to perform black box testing. For black box testing, it is assumed that input, whether it be a user indication or a message from a peer system, can be observed at the time it is consumed by the state machine being tested, and that any outputs, such as a message, can also be observed after it is produced by the state machine being tested. Thus, only inputs and outputs are used to derive a black box test. It is also assumed that the inputs and outputs can be recorded over a period of time, such that one can organize them into a sequence of events to compare with the order of events described in the scenario timeline. Therefore, to pass a test, not only should all inputs and outputs occur, but those inputs and outputs should happen in the same chronological order of the scenario timeline. Any other events or order of events should be considered a failure. In addition to the assumptions and requirements for black box testing, white box testing requires observation of state transitions, the starting, stopping, and expiration of timers, as well as changes in counter values to determine the result of a test. Thus, white box tests require additional checks to verify that after each event (e.g., user indication, reception of a message or timer expiration) all state machine variables contain the proper value based on the changes described in the scenario. These checks are conditional and should evaluate to true or false.

\section{Black Box Testing Example}

*Note: Only state machines A and B are considered for black box testing in the following scenario because the necessary control to ensure that UE B's TFG2 timer expires before UE C's TFG2 timer, which occurs at step 3 of the scenario, may be lacking.

For the scenario, "Call setup - join call" defined in section 2.2.1.1, the following needs to be done:

1. Instantiate two "Basic Group Call” call control state machines, which we will call A and $\mathrm{B}$

2. Interact with the state machines so that state machine B is in a basic group call while A is not

3. Now the following should be observed, in order, after providing an indication to A to "Initiate a basic group call":

a. Observe that A sends a GROUP CALL PROBE message

b. Observe that $B$ receives the GROUP CALL PROBE message

c. Observe that B sends a GROUP CALL ANNOUNCEMENT message with fields coded properly within a certain timer period based on TFG2 + delta.

d. Observe that A receives the GROUP CALL ANNOUNCEMENT message sent by B 
If all events are observed and observed in the order in which they are described, then model has passed the test that was derived from the scenario. If not, then the model has failed the test.

\section{White Box Testing}

For the scenario, "Call setup - join call” defined in section 2.2.1.1, the following needs to be done:

1. Instantiate three "Basic Group Call" call control state machines, which we will call A, $\mathrm{B}$, and $\mathrm{C}$

2. Interact with the state machines so that state machines $B$ and $C$ are in the same basic group call while $\mathrm{A}$ is not

3. Now the following should be observed and checked, in order, after providing an indication to A to "Initiate a basic group call":

a. Observe that A sends a GROUP CALL PROBE message

b. Checks for A:

i. Stored MCPTT group ID matches the group ID

ii. The corresponding Call Type Control state machine exist

iii. The timer TFG1 is running

iv. The timer TFG3 is running

v. Current state is the "S2: waiting for call announcement" state

c. Observe that:

i. $B$ receives the GROUP CALL PROBE message

- Checks for B:

-AND-

a. The timer TFG2 is running

b. The timer TFG6 is running

c. Stored probe response for the call is set to "true"

d. Current state is the "S3: part of ongoing call” state

ii. C receives the GROUP CALL PROBE message

- Checks for C:

a. The timer TFG2 is running

b. The timer TFG6 is running

c. Stored probe response for the call is set to "true"

d. Current state is the "S3: part of ongoing call" state

d. Observe that B's TFG2 timer expires

e. Observe that B sends a GROUP CALL ANNOUNCEMENT message with fields coded properly within a certain timer period based on TFG2 + delta

i. Checks for B:

- $\quad$ The timer TFG2 is running

- $\quad$ The timer TFG6 is running

- Stored probe response for the call is set to "false"

- Current state is the "S3: part of ongoing call" state 
f. Observe that A receives the GROUP CALL ANNOUNCEMENT message sent by B

i. Checks for A:

- $\quad$ The timer TFG6 is running

- The timer TFG2 is running

- $\quad$ The timer TFG1 is NOT running

- $\quad$ The timer TFG3 is NOT running

- The stored SDP matches SDP received in the message

- The stored call ID matches call ID received in the message

- The stored originating user ID matches originating user ID received in the message

- The stored refresh interval matches the refresh interval received in the message

- The stored call start time matches the call start time received in the message

- The floor control state machine has been initialized

- Current state is the "S3: part of ongoing call" state

g. Observe that $\mathrm{C}$ receives the GROUP CALL ANNOUNCEMENT message sent by B

i. Checks for C:

- The timer TFG2 is running

- Stored probe response for the call is set to "false"

If all events are observed, all events are observed in the order in which they are described, and all checks evaluated to true, i.e., all expected values and statuses were found, then the model has passed the test that was derived from the scenario. If not, then the model has failed the test.

- Conformance testing:

To use this scenario for conformance testing, one must examine one UE column at a time.

$\underline{\text { Test } 1}$

Conformance test for UE A (device under test)

First one would need to create a test purpose based on the scenario.

For example: Test purpose: To determine that the Device Under Test (DUT) can join a group call.

Second one would need to create a preamble to place the device under test into the start state stated in the cell in the row labeled with a zero and under the UE A state transition column. For example: Preamble: Turn on device.

Next the first action of conformance test would be to have UE A initiate the joining of a group call and to observe that UE A sends a GROUP CALL PROBE message. This information is contained in the row labeled 1. NOTE: If this was being used as an internal software test, where one has access to the internal information, then one could also confirm 
that there was a state transition from "S1: Start-stop" to "S2: waiting for call announcement" as stated in row 1 column UE A state transition and that timers TFG1 and TFG3 were started as stated in the Consequent behavior column.

If any other message is sent by UE A, then the test would be considered a failure or inconclusive.

Continuing the tester (acting as UE B) would then send a GROUP CALL

ANNOUNCEMENT message properly coded to UE A.

Since there is no observable action by UE A upon the receipt of the GROUP CALL

ANNOUNCEMENT message, one would need to provide an observable ending, such as observe that the UE A hears the transmission on the group that was just joined.

If one hears the transmission of the group call via UE A, then the test verdict is pass.

If this does not occur, then the test verdict is fail.

$\underline{\text { Test } 2}$

Conformance test for UE B (device under test)

First one would need to create a test purpose based on the scenario.

For example: Test purpose: To determine that the DUT responds to a request to join a group call with the properly coded message.

One would read the cell in the row labeled with a zero and the UE B state transition column to determine the start state which is "S3: part of ongoing-call". Thus a preamble would be needed to place UE B (device under test) in this state.

For example: Preamble: Have the tester establish a group call with DUT.

The first action of the conformance test would be to have the tester (acting as UE A) to send a GROUP CALL PROBE message (coded with a different user identifier than the one used in the preamble) to UE B. This information is contained in the row labeled 1.

The tester (acting as UE A) would wait to see if UE B (device under test) sends a GROUP CALL ANNOUNCEMENT message with the properly coded fields back to the tester using the information contained in both row 2 and row 3 Consequent behavior column within a timer value that is TFG2 + delta.

If a GROUP CALL ANNOUNCEMENT message is received with fields properly coded, then the test verdict is pass.

Otherwise fail.

- Interoperability testing:

*Note: Only state machines A and B are considered for interoperability testing in the following scenario because the necessary control to ensure that UE B's TFG2 timer expires before UE C's TFG2 timer, which occurs at step 3 of the scenario, may be lacking.

To use this scenario for interoperability testing, one will consider the observable interfaces between UE A and UE B.

In this case UE A and UE B are required and thus the pair of interfaces is observable. Create a test purpose:

For example: Observe that a UE (UE A) can join an ongoing group call (started by UE B). Preamble: 
o Turn on one UE (UE B)

o Cause UE B to establish a group call

o Begin speaking on the group call

Test step 1: Turn on UE A

Test step 2: Cause UE A to want to join the existing group call.

1: Observe that UE A sends a GROUP CALL PROBE message

2: Observe that UE B sends a GROUP CALL ANNOUNCEMENT message with fields coded properly within a certain timer period based on TFG2 + delta

3: Observe (listen) that UE A hears the conversation on the group call.

If all events are observed, then verdict is pass.

If not, then verdict is fail. 